Supplement of Geosci. Model Dev., 11, 1753-1784, 2018

https://doi.org/10.5194/gmd-11-1753-2018-supplement

(C) Author(s) 2018. This work is distributed under

the Creative Commons Attribution 4.0 License.

(c) (1)

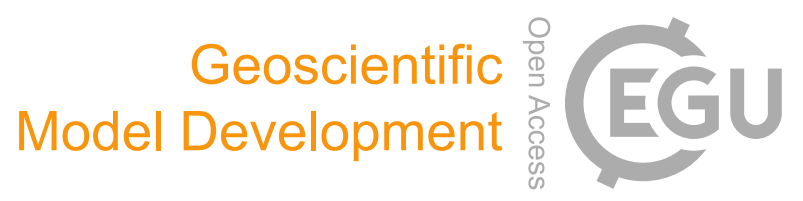

Supplement of

\title{
Impacts of the horizontal and vertical grids on the numerical solutions of the dynamical equations - Part 1: Nonhydrostatic inertia-gravity modes
}

\author{
C. S. Konor and D. A. Randall \\ Correspondence to: Celal S. Konor (csk@atmos.colostate.edu)
}

The copyright of individual parts of the supplement might differ from the CC BY 4.0 License. 


\section{Table of Contents}

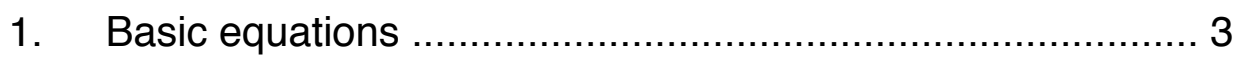

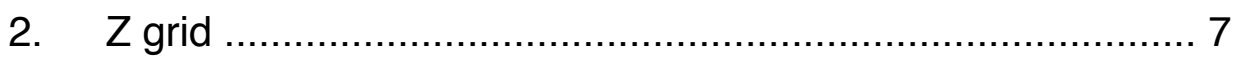

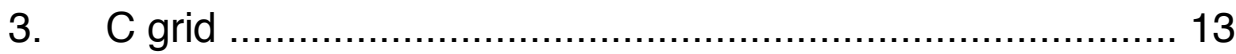

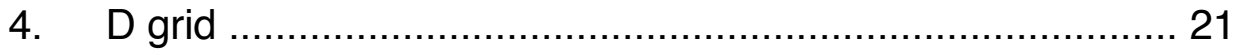

5. D grid (vertical velocity defined at corners) ...................... 30

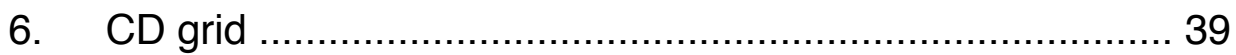

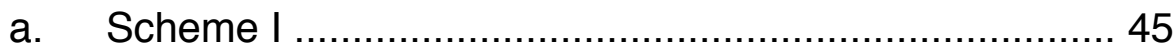

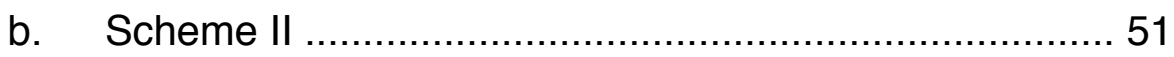

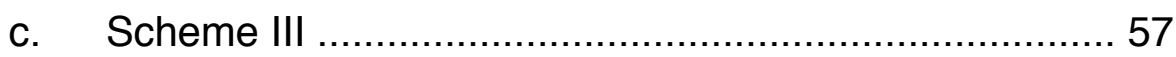

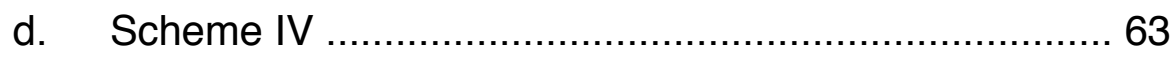

e. Scheme V .......................................................... 70

f. Rossby modes: Scheme I ........................................... 75

g. Rossby modes: Scheme II .......................................... 89

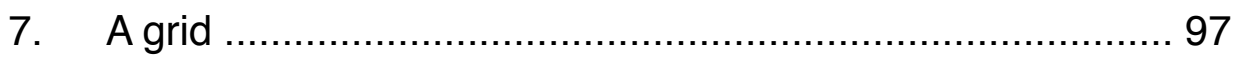

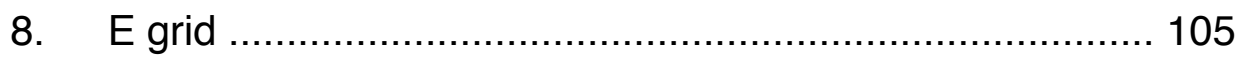

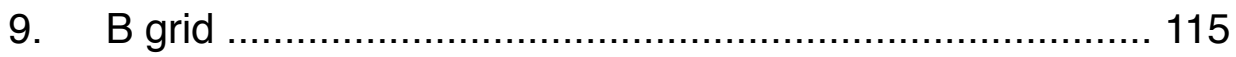

10. Numerical solutions and computational modes ............... 124

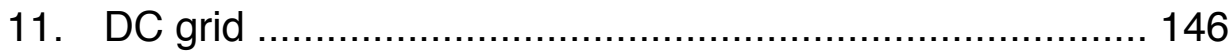

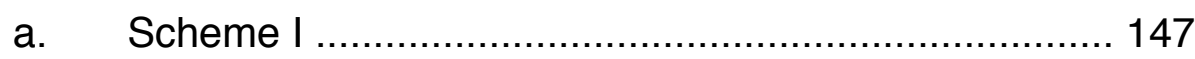

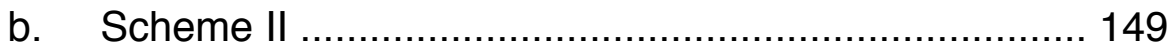

c. Numerical solutions of gravity modes (DC-grid).......... 154

d. Rossby modes ....................................................... 159

12. Anelastic, quasi-hydrostatic and Lamb wave (shallow-water) solutions: Nonhydrostatic effects............. 162

13. Baroclinic Rossby Modes with the fully compressible, quasi-hydrostatic and anelastic systems.......................... 175

14. Numerical simulations with noisy forcing......................... 180

15. Horizontal momentum modes with the A, E, and B grids.. 186 


\section{Basic Linearized Anelastic Equations}

\section{Momentum equations}

Linearized anelastic equations of Lipps and Hemler (1982).

Zonal component of the momentum:

$$
\frac{\partial u}{\partial t}=-\frac{\partial P}{\partial x}+f v
$$

where $P \equiv c_{p} \theta \pi^{\prime}$.

Meridional component of the momentum:

$$
\frac{\partial v}{\partial t}=-\frac{\partial P}{\partial y}-f u
$$

Vertical momentum equation:

$$
\frac{\partial w}{\partial t}=-\frac{\partial P}{\partial z}+B
$$

where

$$
B \equiv g \frac{\theta^{\prime}}{\theta_{0}}
$$

Buoyancy (thermodynamic) equation:

$$
\frac{\partial B}{\partial t}=-N^{2} w
$$

where

$$
N^{2} \equiv \frac{g}{\theta_{0}} \frac{\partial \theta_{0}}{\partial z}
$$

Mass continuity equation: 


$$
\frac{\partial}{\partial x}\left(\rho_{0} u\right)+\frac{\partial}{\partial y}\left(\rho_{0} v\right)+\frac{\partial}{\partial z}\left(\rho_{0} w\right)=0
$$

The vorticity:

$$
\omega_{z} \equiv \frac{\partial v}{\partial x}-\frac{\partial u}{\partial y}
$$

The divergence:

$$
D \equiv \frac{\partial u}{\partial x}+\frac{\partial v}{\partial y}
$$

From $\partial / \partial x(1.2)-\partial / \partial y(1.1)$ :

$$
\frac{\partial \omega_{z}}{\partial t}=-f D
$$

From $\partial / \partial x(1.1)+\partial / \partial y(1.2)$ :

$$
\frac{\partial D}{\partial t}=f \omega_{z}-\left(\frac{\partial^{2}}{\partial x^{2}}+\frac{\partial^{2}}{\partial y^{2}}\right) P
$$

Vertical momentum equation:

$$
\frac{\partial w}{\partial t}=-\frac{\partial P}{\partial z}+B
$$

Buoyancy (thermodynamic) equation:

$$
\frac{\partial B}{\partial t}=-N^{2} w
$$

Mass continuity equation:

$$
D+\left(\frac{\partial}{\partial z}+\frac{1}{\rho_{0}} \frac{\partial \rho_{0}}{\partial z}\right) w=0
$$

Multiplying equations by $\rho_{0}^{1 / 2}(z)$ : 


$$
\begin{gathered}
\frac{\partial\left(\rho_{0}^{1 / 2} \omega_{z}\right)}{\partial t}=-f\left(\rho_{0}^{1 / 2} D\right) \\
\frac{\partial\left(\rho_{0}^{1 / 2} D\right)}{\partial t}=f\left(\rho_{0}^{1 / 2} \omega_{z}\right)-\left(\frac{\partial^{2}}{\partial x^{2}}+\frac{\partial^{2}}{\partial y^{2}}\right)\left(\rho_{0}^{1 / 2} P\right) \\
\frac{\partial\left(\rho_{0}^{1 / 2} w\right)}{\partial t}=-\left(\frac{\partial}{\partial z}-\frac{1}{2 \rho_{0}} \frac{\partial \rho_{0}}{\partial z}\right)\left(\rho_{0}^{1 / 2} P\right)+\left(\rho_{0}^{1 / 2} B\right) \\
\frac{\partial\left(\rho_{0}^{1 / 2} B\right)}{\partial t}=-N^{2}\left(\rho_{0}^{1 / 2} w\right) \\
\left(\rho_{0}^{1 / 2} D\right)+\left(\frac{\partial}{\partial z}+\frac{1}{2 \rho_{0}} \frac{\partial \rho_{0}}{\partial z}\right)\left(\rho_{0}^{1 / 2} w\right)=0
\end{gathered}
$$

By replacing $\rho_{0}^{1 / 2} \omega_{z}$ by $\omega_{z}$ and replacing others likewise.

The linearized anelastic equations used in the paper:

$$
\begin{gathered}
\frac{\partial \omega_{z}}{\partial t}=-f D \\
\frac{\partial D}{\partial t}=f \omega_{z}-\left(\frac{\partial^{2}}{\partial x^{2}}+\frac{\partial^{2}}{\partial y^{2}}\right) P \\
\frac{\partial w}{\partial t}=-\left(\frac{\partial}{\partial z}-\frac{1}{2 \rho_{0}} \frac{\partial \rho_{0}}{\partial z}\right) P+B \\
\frac{\partial B}{\partial t}=-N^{2} w \\
D+\left(\frac{\partial}{\partial z}+\frac{1}{2 \rho_{0}} \frac{\partial \rho_{0}}{\partial z}\right) w=0
\end{gathered}
$$

\section{Baroclinic equations governing Rossby modes:}


Quasi-geostrophic anelastic equations with the quasi-static assumption on a midlatitude betaplane:

Time change of divergence and vertical momentum are ignored.

$$
\begin{gathered}
\frac{\partial \omega_{z}}{\partial t}=-f_{0} D-\frac{\beta}{f_{0}} \frac{\partial P}{\partial x} \\
0=f_{0} \omega_{z}-\left(\frac{\partial^{2}}{\partial x^{2}}+\frac{\partial^{2}}{\partial y^{2}}\right) P \\
0=-\left(\frac{\partial}{\partial z}-\frac{1}{2 \rho_{0}} \frac{\partial \rho_{0}}{\partial z}\right) P+B \\
\frac{\partial B}{\partial t}=-N^{2} w \\
D+\left(\frac{\partial}{\partial z}+\frac{1}{2 \rho_{0}} \frac{\partial \rho_{0}}{\partial z}\right) w=0
\end{gathered}
$$

\section{Barotropic equations governing Rossby modes:}

Divergence and vertical momentum is ignored.

$$
\begin{gathered}
\frac{\partial \omega_{z}}{\partial t}=-\frac{\beta}{f_{0}} \frac{\partial P}{\partial x} \\
0=f_{0} \omega_{z}-\left(\frac{\partial^{2}}{\partial x^{2}}+\frac{\partial^{2}}{\partial y^{2}}\right) P \\
0=-\left(\frac{\partial}{\partial z}-\frac{1}{2 \rho_{0}} \frac{\partial \rho_{0}}{\partial z}\right) P
\end{gathered}
$$

Eq. (1.30) indicates that $P$ is vertically uniform. 


\section{Z-grid}

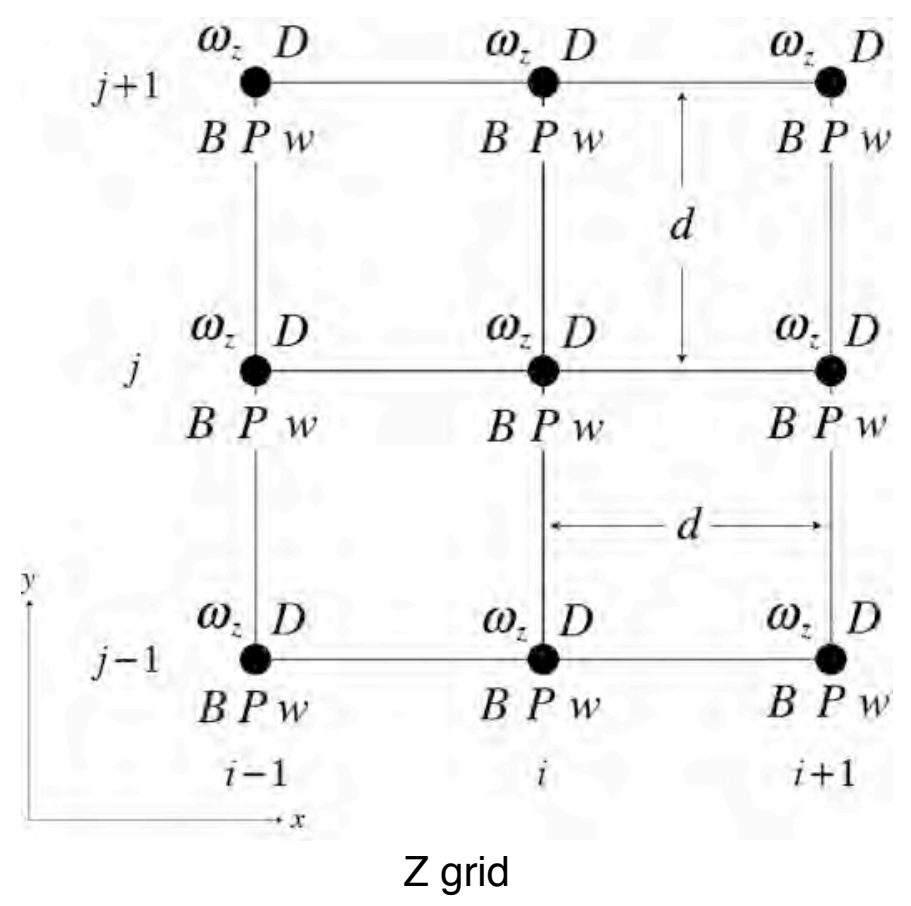

\section{Inertia-gravity modes}

Vorticity equation from (M.12):

$$
\frac{\partial\left(\omega_{z}\right)_{i, j}}{\partial t}=-f D_{i, j}
$$

Divergence equation from (M.8):

$$
\frac{\partial}{\partial t} D_{i, j}=-\frac{1}{d^{2}}\left(P_{i+1, j}+P_{i-1, j}+P_{i, j+1}+P_{i, j-1}-4 P_{i, j}\right)+f\left(\omega_{z}\right)_{i, j}
$$

Vertical momentum equation: 


$$
\frac{\partial w_{i, j}}{\partial t}=-\left[\frac{\partial}{\partial z}-\left(\frac{1}{2 \rho_{0}} \frac{\partial \rho_{0}}{\partial z}\right)\right] P_{i, j}+B_{i, j}
$$

Thermodynamic equation:

$$
\frac{\partial B_{i, j}}{\partial t}=-N^{2} w_{i, j}
$$

Mass continuity equation:

$$
D_{i, j}+\left[\frac{\partial}{\partial z}+\left(\frac{1}{2 \rho_{0}} \frac{\partial \rho_{0}}{\partial z}\right)\right] w_{i, j}=0
$$

From (4)-(6):

$$
\left(\frac{\partial^{2}}{\partial t^{2}}+N^{2}\right) D_{i, j}-\left[\frac{\partial^{2}}{\partial z^{2}}-\left(\frac{1}{2 \rho_{0}} \frac{\partial \rho_{0}}{\partial z}\right)^{2}\right] \frac{\partial}{\partial t} P_{i, j}=0
$$

Define:

$\phi_{i, j}(z, t) \equiv \operatorname{Re}\left\{\hat{\Phi} e^{i(k d i+\ell d j+m z-v t)}\right\} \quad$ and $\quad \phi_{i+1 / 2, j+1 / 2}(z, t) \equiv \operatorname{Re}\left\{\hat{\Phi} e^{i[k d(i+1 / 2)+\ell d(j+1 / 2)+m z-v t]}\right\}$

Using (8) in (1):

$$
-i v \hat{\omega}_{z}=-f \hat{D}
$$

Using (8) in (2):

$$
-\underset{\sim}{i} v \hat{D}=f \hat{\omega}_{z}-\left(\frac{e^{i k d}+e^{-i k d}+e^{i \ell d}+e^{-i \ell d}-4}{d^{2}}\right) \hat{P}
$$

Using (8) in (7): 


$$
\left(-v^{2}+N^{2}\right) \hat{D}-\left[m^{2}+\left(\frac{1}{2 \rho_{0}} \frac{\partial \rho_{0}}{\partial z}\right)^{2}\right] i v P_{i, j}=0
$$

$\ln (10)$ :

$$
\begin{aligned}
\frac{e^{i k d}+e^{-i k d}+e^{i \ell d}+e^{-i \ell d}-4}{d^{2}} & =\frac{2 \cos (k d)+2 \cos (\ell d)-4}{d^{2}} \\
= & \left(\frac{-\sin ^{2}\left(\frac{1}{2} k d\right)}{\left(\frac{1}{2} k d\right)^{2}} k^{2}+\frac{-\sin ^{2}\left(\frac{1}{2} \ell d\right)}{\left(\frac{1}{2} \ell d\right)^{2}} \ell^{2}\right)
\end{aligned}
$$

Rewrite (13):

$$
\frac{e^{i k d}+e^{-i k d}+e^{i \ell d}+e^{-i \ell d}-4}{d^{2}}=-\xi^{2} k^{2}-\eta^{2} \ell^{2}
$$

where

$$
\xi \equiv \frac{\sin \left(\frac{1}{2} k d\right)}{\frac{1}{2} k d} \quad \text { and } \quad \eta \equiv \frac{\sin \left(\frac{1}{2} \ell d\right)}{\frac{1}{2} \ell d}
$$

Using (12) and (14a) in (9)-(11):

$$
\begin{gathered}
-i v \hat{\omega}_{z}=-f \hat{D} \\
-i v \hat{D}=\left(\xi^{2} k^{2}+\eta^{2} \ell^{2}\right) \hat{P}+f \hat{\omega}_{z} \\
\left(-v^{2}+N^{2}\right) \hat{D}+\left[\frac{\partial^{2}}{\partial z^{2}}-\left(\frac{1}{2 \rho_{0}} \frac{\partial \rho_{0}}{\partial z}\right)^{2}\right] i v \hat{P}=0
\end{gathered}
$$

From (20) and (21):

$$
\left(v^{2}-\mu^{2} f^{2}\right) \hat{D}=\left(\xi^{2} k^{2}+\eta^{2} \ell^{2}\right) i v \hat{P}
$$

From (22) and (23): 


$$
v^{2}=\frac{N^{2}\left(\xi^{2} k^{2}+\eta^{2} \ell^{2}\right)+f^{2}\left(m^{2}+\frac{1}{4 H^{2}}\right)}{\left(\xi^{2} k^{2}+\eta^{2} \ell^{2}\right)+\left(m^{2}+\frac{1}{4 H^{2}}\right)}
$$

\section{Rossby modes}

Baroclinic modes:

Adding the beta effect to the vorticity equation (1):

$$
\frac{\partial\left(\omega_{z}\right)_{i, j}}{\partial t}=-f_{0} D_{i, j}-\frac{\beta}{f_{0}} \frac{P_{i+1, j}-P_{i-1, j}}{2 d}
$$

Dropping $\partial D / \partial t$ in (2):

$$
0=-\frac{1}{d^{2}}\left(P_{i+1, j}+P_{i-1, j}+P_{i, j+1}+P_{i, j-1}-4 P_{i, j}\right)+f\left(\omega_{z}\right)_{i, j}
$$

Dropping $\partial^{2} D / \partial t^{2}$ in (7):

$$
N^{2} D_{i, j}-\left[\frac{\partial^{2}}{\partial z^{2}}-\left(\frac{1}{2 \rho_{0}} \frac{\partial \rho_{0}}{\partial z}\right)^{2}\right] \frac{\partial}{\partial t} P_{i, j}=0
$$

From (30)-(32):

$$
\begin{gathered}
-i v \hat{\omega}_{z}=-f_{0} \hat{D}-\frac{\beta}{f_{0}} \frac{e^{i k d}-e^{-i k d}}{2 d} \hat{P} \\
0=\left(\xi^{2} k^{2}+\eta^{2} \ell^{2}\right) \hat{P}+f_{0} \hat{\omega}_{z} \\
N^{2} \hat{D}-\left[m^{2}+\left(\frac{1}{2 \rho_{0}} \frac{\partial \rho_{0}}{\partial z}\right)^{2}\right] i v \hat{P}=0
\end{gathered}
$$

Rewrite (40): 


$$
-\underset{\sim}{i} \hat{\omega_{z}}=-f_{0} \hat{D}-\underset{\sim}{i} \frac{\beta}{f_{0}} \frac{2 \sin (k d)}{2 d} \hat{P}
$$

Rewrite (43):

$$
-\underset{\sim}{v} v \hat{\omega}_{z}=-f_{0} \hat{D}-\underset{\sim}{i} \frac{\beta}{f_{0}} \tilde{\xi}_{k} \hat{P}
$$

where

$$
\tilde{\xi} \equiv \frac{\sin (k d)}{k d}
$$

From (41), (42) and (44):

$$
v N^{2}\left(\xi^{2} k^{2}+\eta^{2} \ell^{2}\right) \hat{P}=-f_{0}^{2}\left[m^{2}+\left(\frac{1}{2 \rho_{0}} \frac{\partial \rho_{0}}{\partial z}\right)^{2}\right] v \hat{P}-N^{2} \beta \tilde{\xi}_{k} \hat{P}
$$

Using $\frac{1}{\rho_{0}} \frac{\partial \rho_{0}}{\partial z}=-\frac{1}{H}$ in (46) and for nontrivial solutions $(\hat{P} \neq 0)$ :

$$
v=\frac{-\beta \tilde{\xi}_{k}}{\left(\xi^{2} k^{2}+\eta^{2} \ell^{2}\right)+\frac{f_{0}^{2}}{N^{2}}\left(m^{2}+\frac{1}{4 H}\right)}
$$

Barotropic modes:

Assuming $D=0$ in (30)

$$
\frac{\partial\left(\omega_{z}\right)_{i, j}}{\partial t}=-\frac{\beta}{f_{0}} \frac{P_{i+1, j}-P_{i-1, j}}{2 d}
$$

Writing (31) again:

$$
0=-\frac{1}{d^{2}}\left(P_{i+1, j}+P_{i-1, j}+P_{i, j+1}+P_{i, j-1}-4 P_{i, j}\right)+f\left(\omega_{z}\right)_{i, j}
$$


Assuming $D=0$ in (32)

$$
-\left[\frac{\partial^{2}}{\partial z^{2}}-\left(\frac{1}{2 \rho_{0}} \frac{\partial \rho_{0}}{\partial z}\right)^{2}\right] \frac{\partial}{\partial t} P_{i, j}=0
$$

( $\mathrm{P}$ is vertically constant).

From (50) and (51):

$$
0=v\left(\xi^{2} k^{2}+\eta^{2} \ell^{2}\right) \hat{P}+\beta \tilde{\xi}_{k} \hat{P}
$$

From (53) and for nontrivial solutions $(\hat{P} \neq 0)$ :

$$
v=\frac{-\beta \tilde{\xi}_{k}}{\xi^{2} k^{2}+\eta^{2} \ell^{2}}
$$




\section{C-grid}

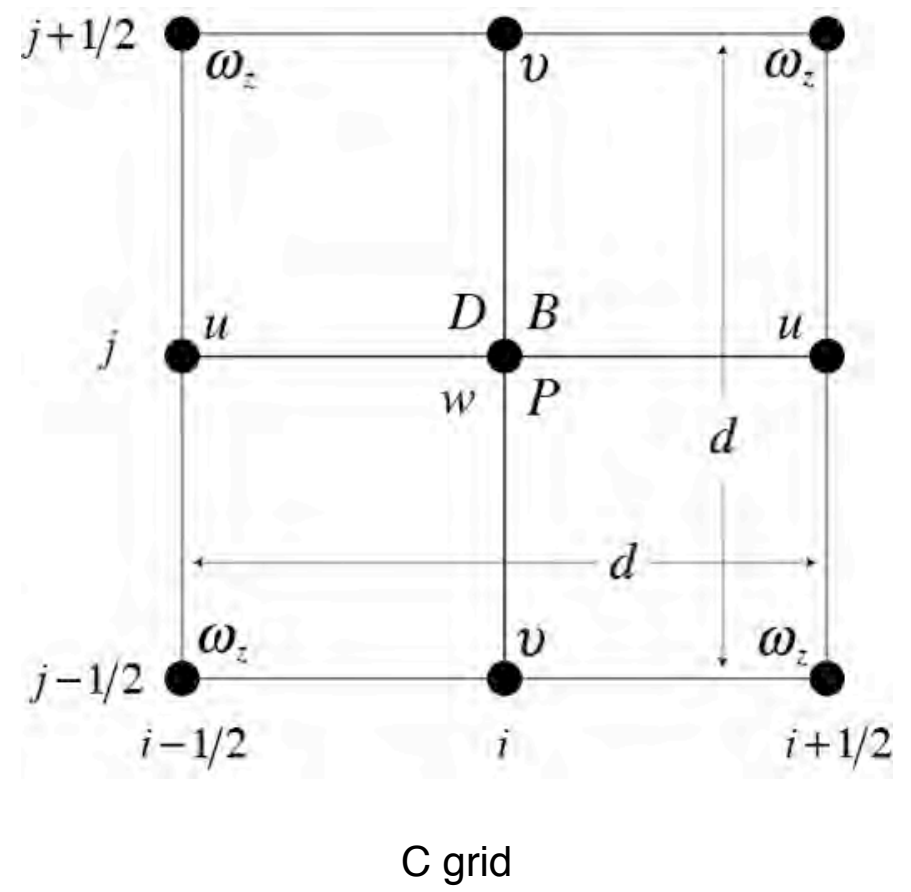

\section{Inertia-gravity modes}

Horizontal momentum equation:

$$
\begin{aligned}
& \frac{\partial u_{i+1 / 2, j}}{\partial t}=-\frac{P_{i+1, j}-P_{i, j}}{d}+f \bar{v}_{i+1 / 2, j} \\
& \frac{\partial v_{i, j+1 / 2}}{\partial t}=-\frac{P_{i, j+1}-P_{i, j}}{d}-f \bar{u}_{i, j+1 / 2}
\end{aligned}
$$

where

$$
\begin{aligned}
& \bar{v}_{i+1 / 2, j} \equiv \frac{1}{4}\left(v_{i, j-1 / 2}+v_{i+1, j-1 / 2}+v_{i, j+1 / 2}+v_{i+1, j+1 / 2}\right) \\
& \bar{u}_{i, j+1 / 2} \equiv \frac{1}{4}\left(u_{i+1 / 2, j}+u_{i-1 / 2, j}+u_{i+1 / 2, j+1}+u_{i-1 / 2, j+1}\right)
\end{aligned}
$$


To derive divergence equation for center points $(i, j)$ :

$$
\begin{aligned}
& \frac{\partial}{\partial t}\left(\frac{u_{i+1 / 2, j}-u_{i-1 / 2, j}}{d}\right)=-\frac{P_{i+1, j}-2 P_{i, j}+P_{i-1, j}}{d^{2}}+f\left(\frac{\bar{v}_{i+1 / 2, j}-\bar{v}_{i-1 / 2, j}}{d}\right) \\
& \frac{\partial}{\partial t}\left(\frac{v_{i, j+1 / 2}-v_{i, j-1 / 2}}{d}\right)=-\frac{P_{i, j+1}-2 P_{i, j}+P_{i, j-1}}{d^{2}}-f\left(\frac{\bar{u}_{i, j+1 / 2}-\bar{u}_{i, j-1 / 2}}{d}\right)
\end{aligned}
$$

Define:

$$
D_{i, j} \equiv \frac{1}{d}\left(u_{i+1 / 2, j}-u_{i-1 / 2, j}+v_{i, j+1 / 2}-v_{i, j-1 / 2}\right)
$$

Divergence equation (1):

$$
\frac{\partial}{\partial t} D_{i, j}=-\frac{P_{i+1, j}+P_{i-1, j}+P_{i, j+1}+P_{i, j-1}-4 P_{i, j}}{d^{2}}+f\left(\frac{\bar{v}_{i+1 / 2, j}-\bar{v}_{i-1 / 2, j}}{d}-\frac{\bar{u}_{i, j+1 / 2}-\bar{u}_{i, j-1 / 2}}{d}\right)
$$

Define:

$$
\left(\omega_{z}\right)_{i+1 / 2, j+1 / 2} \equiv \frac{1}{d}\left(v_{i+1 / 2, j+1}-v_{i+1 / 2, j}-u_{i+1 / 2, j+1}+u_{i+1 / 2, j}\right)
$$

Divergence equation (2):

Consider (M.6) and (M.7):

$$
\frac{\partial}{\partial t} D_{i, j}=-\frac{P_{i+1, j}+P_{i-1, j}+P_{i, j+1}+P_{i, j-1}-4 P_{i, j}}{d^{2}}+f \frac{1}{4}\left[\left(\omega_{z}\right)_{i+1 / 2, j+1 / 2}+\left(\omega_{z}\right)_{i-1 / 2, j+1 / 2}+\left(\omega_{z}\right)_{i+1 / 2, j-1 / 2}+\left(\omega_{z}\right)_{i-1 / 2, j-1 / 2}\right]
$$

To derive vorticity equation for center point $(i+1 / 2, j+1 / 2)$ : 


$$
\begin{aligned}
& \frac{\partial}{\partial t}\left(\frac{v_{i+1, j+1 / 2}-v_{i, j+1 / 2}}{d}\right)=-\frac{P_{i+1, j+1}-P_{i+1, j}-P_{i, j+1}+P_{i, j}}{d^{2}}-f\left(\frac{\bar{u}_{i+1, j+1 / 2}-\bar{u}_{i, j+1 / 2}}{d}\right) \\
& \frac{\partial}{\partial t}\left(\frac{-u_{i+1 / 2, j+1}+u_{i+1 / 2, j}}{d}\right)=-\frac{-P_{i+1, j+1}+P_{i, j+1}+P_{i+1, j}-P_{i, j}}{d^{2}}-f\left(\frac{\bar{v}_{i+1 / 2, j+1}-\bar{v}_{i+1 / 2, j}}{d}\right)
\end{aligned}
$$

The vorticity equation (1):

$\frac{\partial}{\partial t}\left(\frac{v_{i+1, j+1 / 2}-v_{i, j+1 / 2}-u_{i+1 / 2, j+1}+u_{i+1 / 2, j}}{d}\right)=-f\left(\frac{\bar{u}_{i+1, j+1 / 2}-\bar{u}_{i, j+1 / 2}+\bar{v}_{i+1 / 2, j+1}-\bar{v}_{i+1 / 2, j}}{d}\right)$

The vorticity equation (2):

Using (M.7) and (M.5) in (M.11):

$$
\frac{\partial}{\partial t}\left(\omega_{z}\right)_{i+1 / 2, j+1 / 2}=-f \frac{1}{4}\left(D_{i+1, j+1}+D_{i+1, j}+D_{i, j+1}+D_{i, j}\right)
$$

Vorticity equation from (M.12):

$$
\frac{\partial}{\partial t}\left(\omega_{z}\right)_{i+1 / 2, j+1 / 2}=-f \frac{1}{4}\left(D_{i+1, j+1}+D_{i+1, j}+D_{i, j+1}+D_{i, j}\right)
$$

Divergence equation from (M.8):

$$
\frac{\partial}{\partial t} D_{i, j}=-\frac{P_{i+1, j}+P_{i-1, j}+P_{i, j+1}+P_{i, j-1}-4 P_{i, j}}{d^{2}}+f \frac{1}{4}\left[\left(\omega_{z}\right)_{i+1 / 2, j+1 / 2}+\left(\omega_{z}\right)_{i-1 / 2, j+1 / 2}+\left(\omega_{z}\right)_{i+1 / 2, j-1 / 2}+\left(\omega_{z}\right)_{i-1 / 2, j-1 / 2}\right]
$$

Vertical momentum equation:

$$
\frac{\partial w_{i, j}}{\partial t}=-\left[\frac{\partial}{\partial z}-\left(\frac{1}{2 \rho_{0}} \frac{\partial \rho_{0}}{\partial z}\right)\right] P_{i, j}+B_{i, j}
$$

Thermodynamic equation: 


$$
\frac{\partial B_{i, j}}{\partial t}=-N^{2} w_{i, j}
$$

Mass continuity equation:

$$
D_{i, j}+\left[\frac{\partial}{\partial z}+\left(\frac{1}{2 \rho_{0}} \frac{\partial \rho_{0}}{\partial z}\right)\right] w_{i, j}=0
$$

From (4)-(6):

$$
\left(\frac{\partial^{2}}{\partial t^{2}}+N^{2}\right) D_{i, j}-\left[\frac{\partial^{2}}{\partial z^{2}}-\left(\frac{1}{2 \rho_{0}} \frac{\partial \rho_{0}}{\partial z}\right)^{2}\right] \frac{\partial}{\partial t} P_{i, j}=0
$$

Define:

$\phi_{i, j}(z, t) \equiv \operatorname{Re}\left\{\hat{\Phi} e^{i(k d i+\ell d j+m z-v t)}\right\} \quad$ and $\quad \phi_{i+1 / 2, j+1 / 2}(z, t) \equiv \operatorname{Re}\left\{\hat{\Phi} e^{i[k d(i+1 / 2)+\ell d(j+1 / 2)+m z-v t]}\right\}$

Using (8) in (1):

$$
-i v \hat{\omega}_{z}=-f \frac{1}{4}\left(e^{-i k \frac{d}{2}} e^{-i \ell \frac{d}{2}}+e^{i k \frac{d}{2}} e^{-i \ell \frac{d}{2}}+e^{-i k \frac{d}{2}} e^{i \ell \ell \frac{d}{2}}+e^{i k \frac{d}{2}} e^{i \ell \frac{d}{2}}\right) \hat{D}
$$

Using (8) in (2):

$$
-\underset{\sim}{i} v \hat{D}=f \frac{1}{4}\left(e^{i k \frac{d}{2}} e^{i \ell \frac{d}{2}}+e^{-i k \frac{d}{2}} e^{i \ell \ell \frac{d}{2}}+e^{i k \frac{d}{2}} e^{-i \ell \frac{d}{2}}+e^{-i k \frac{d}{2}} e^{-i \ell \frac{d}{2}}\right) \hat{\omega}_{z}-\left(\frac{e^{i k d}+e^{-i k d}+e^{i \ell d}+e^{-i \ell d}-4}{d^{2}}\right) \hat{P}
$$

Using (8) in (7):

$$
\left(-v^{2}+N^{2}\right) \hat{D}-\left[m^{2}+\left(\frac{1}{2 \rho_{0}} \frac{\partial \rho_{0}}{\partial z}\right)^{2}\right] i v P_{i, j}=0
$$


$\ln (9)-(10)$ :

$$
\begin{aligned}
\mu & \equiv \frac{1}{4}\left(e^{-i k \frac{d}{2}} e^{-i \ell \frac{d}{2}}+e^{i k \frac{d}{2}} e^{-i \ell \frac{d}{2}}+e^{-i k \frac{d}{2}} e^{i \ell \frac{d}{2}}+e^{i k \frac{d}{2}} e^{i \ell \frac{d}{2}}\right) \\
& =\frac{1}{4}\left[\left(e^{-i k \frac{d}{2}}+e^{i k \frac{d}{2}}\right)\left(e^{-i \ell \frac{d}{2}}+e^{i \ell \frac{d}{2}}\right)\right]=\cos \left(k \frac{d}{2}\right) \cos \left(\ell \frac{d}{2}\right)
\end{aligned}
$$

$\ln (10)$ :

$$
\begin{aligned}
\frac{e^{i k d}+e^{-i k d}+e^{i \ell d}+e^{-i \ell l d}-4}{d^{2}} & =\frac{2 \cos (k d)+2 \cos (\ell d)-4}{d^{2}} \\
& =\left(\frac{-\sin ^{2}\left(\frac{1}{2} k d\right)}{\left(\frac{1}{2} k d\right)^{2}} k^{2}+\frac{-\sin ^{2}\left(\frac{1}{2} \ell d\right)}{\left(\frac{1}{2} \ell d\right)^{2}} \ell^{2}\right)
\end{aligned}
$$

Rewrite (13):

$$
\frac{e^{i k d}+e^{-i k d}+e^{i \ell d}+e^{-i \ell d}-4}{d^{2}}=-\xi^{2} k^{2}-\eta^{2} \ell^{2}
$$

where

$$
\xi \equiv \frac{\sin \left(\frac{1}{2} k d\right)}{\frac{1}{2} k d} \quad \text { and } \quad \eta \equiv \frac{\sin \left(\frac{1}{2} \ell d\right)}{\frac{1}{2} \ell d}
$$

Using (12) and (14a) in (9)-(10) and writing (11) again:

$$
\begin{gathered}
-i v \hat{\omega}_{z}=-\mu f \hat{D} \\
-i v \hat{D}=\left(\xi^{2} k^{2}+\eta^{2} \ell^{2}\right) \hat{P}+\mu f \hat{\omega}_{z} \\
\left(-v^{2}+N^{2}\right) \hat{D}+\left[\frac{\partial^{2}}{\partial z^{2}}-\left(\frac{1}{2 \rho_{0}} \frac{\partial \rho_{0}}{\partial z}\right)^{2}\right] i v \hat{P}=0
\end{gathered}
$$

From (20) and (21):

$$
\left(v^{2}-\mu^{2} f^{2}\right) \hat{D}=\left(\xi^{2} k^{2}+\eta^{2} \ell^{2}\right) i v \hat{P}
$$


From (22) and (23):

$$
v^{2}=\frac{N^{2}\left(\xi^{2} k^{2}+\eta^{2} \ell^{2}\right)+\mu^{2} f^{2}\left(m^{2}+\frac{1}{4 H^{2}}\right)}{\left(\xi^{2} k^{2}+\eta^{2} \ell^{2}\right)+\left(m^{2}+\frac{1}{4 H^{2}}\right)}
$$

\section{Rossby modes}

Baroclinic modes:

Adding the beta effect to the vorticity equation (1):

$$
\frac{\partial\left(\omega_{z}\right)_{i+1 / 2, j+1 / 2}}{\partial t}=-f_{0} \frac{1}{4}\left(D_{i+1, j+1}+D_{i+1, j}+D_{i, j+1}+D_{i, j}\right)-\frac{\beta}{f_{0}} \frac{\bar{P}_{i+3 / 2, j+1 / 2}-\bar{P}_{i-1 / 2, j+1 / 2}}{2 d}
$$

Dropping $\partial D / \partial t$ in (2):

$$
0=-\frac{P_{i+1, j}+P_{i-1, j}+P_{i, j+1}+P_{i, j-1}-4 P_{i, j}}{d^{2}}+f_{0} \frac{1}{4}\left[\left(\omega_{z}\right)_{i+1 / 2, j+1 / 2}+\left(\omega_{z}\right)_{i-1 / 2, j+1 / 2}+\left(\omega_{z}\right)_{i+1 / 2, j-1 / 2}+\left(\omega_{z}\right)_{i-1 / 2, j-1 / 2}\right]
$$

Dropping $\partial^{2} D / \partial t^{2}$ in (7):

$$
N^{2} D_{i, j}-\left[\frac{\partial^{2}}{\partial z^{2}}-\left(\frac{1}{2 \rho_{0}} \frac{\partial \rho_{0}}{\partial z}\right)^{2}\right] \frac{\partial}{\partial t} P_{i, j}=0
$$

From (30)-(32):

$$
\begin{gathered}
-\underset{\sim}{i v \hat{\omega}_{z}}=-\mu f_{0} \hat{D}-\mu \frac{\beta}{f_{0}} \frac{e^{i k d}-e^{-i k d}}{2 d} \hat{P} \\
0=\left(\xi^{2} k^{2}+\eta^{2} \ell^{2}\right) \hat{P}+\mu f_{0} \hat{\omega}_{z}
\end{gathered}
$$




$$
\mu \hat{D} N^{2}-\left[m^{2}+\left(\frac{1}{2 \rho_{0}} \frac{\partial \rho_{0}}{\partial z}\right)^{2}\right] \underset{\sim}{i} v \hat{P}=0
$$

Rewrite (40):

$$
-\underset{\sim}{i v \hat{\omega}_{z}}=-\mu f_{0} \hat{D}-\underset{\sim}{i} \mu \frac{\beta}{f_{0}} \frac{2 \sin (k d)}{2 d} \hat{P}
$$

Rewrite (43):

$$
-\underset{\sim}{i} v \hat{\omega}_{z}=-\mu f_{0} \hat{D}-\underset{\sim}{i} \mu \frac{\beta}{f_{0}} \tilde{\xi}_{k} \hat{P}
$$

where

$$
\tilde{\xi} \equiv \frac{\sin (k d)}{k d}
$$

From (41), (42) and (44):

$$
v N^{2}\left(\xi^{2} k^{2}+\eta^{2} \ell^{2}\right) \hat{P}=-f_{0}^{2}\left[m^{2}+\left(\frac{1}{2 \rho_{0}} \frac{\partial \rho_{0}}{\partial z}\right)^{2}\right] v \hat{P}-\underset{\sim}{i} \mu^{2} N^{2} \beta \tilde{\xi} k \hat{P}
$$

Using $\frac{1}{\rho_{0}} \frac{\partial \rho_{0}}{\partial z}=-\frac{1}{H}$ in (46) and for nontrivial solutions $(\hat{P} \neq 0)$ :

$$
v=\frac{-\mu^{2} \beta \tilde{\xi}_{k}}{\left(\xi^{2} k^{2}+\eta^{2} \ell^{2}\right)+\frac{f_{0}^{2}}{N^{2}}\left(m^{2}+\frac{1}{4 H}\right)}
$$

Barotropic modes:

Assuming $D=0$ in (30)

$$
\frac{\partial\left(\omega_{z}\right)_{i+1 / 2, j+1 / 2}}{\partial t}=-\frac{\beta}{f_{0}} \frac{\bar{P}_{i+3 / 2, j+1 / 2}-\bar{P}_{i-1 / 2, j+1 / 2}}{2 d}
$$

Writing (31) again: 
$0=-\frac{P_{i+1, j}+P_{i-1, j}+P_{i, j+1}+P_{i, j-1}-4 P_{i, j}}{d^{2}}+f_{0} \frac{1}{4}\left[\left(\omega_{z}\right)_{i+1 / 2, j+1 / 2}+\left(\omega_{z}\right)_{i-1 / 2, j+1 / 2}+\left(\omega_{z}\right)_{i+1 / 2, j-1 / 2}+\left(\omega_{z}\right)_{i-1 / 2, j-1 / 2}\right]$

Assuming $D=0$ in (32)

$$
-\left[\frac{\partial^{2}}{\partial z^{2}}-\left(\frac{1}{2 \rho_{0}} \frac{\partial \rho_{0}}{\partial z}\right)^{2}\right] \frac{\partial}{\partial t} P_{i, j}=0
$$

( $\mathrm{P}$ is vertically constant).

From (50) and (51):

$$
0=v\left(\xi^{2} k^{2}+\eta^{2} \ell^{2}\right) \hat{P}+\mu^{2} \beta \tilde{\xi}_{k} \hat{P}
$$

From (53) and for nontrivial solutions $(\hat{P} \neq 0)$ :

$$
v=\frac{-\mu^{2} \beta \tilde{\xi}_{k}}{\xi^{2} k^{2}+\eta^{2} \ell^{2}}
$$




\section{D-grid}

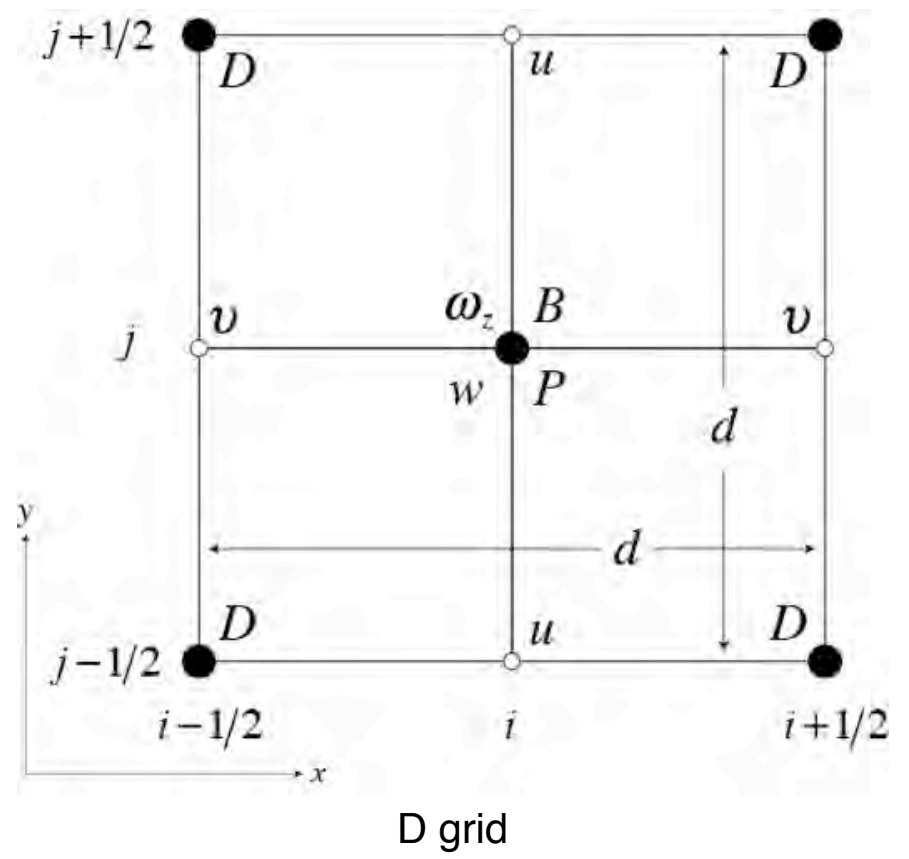

\section{Inertia-gravity modes}

Horizontal momentum equation:

$$
\begin{aligned}
& \frac{\partial u_{i, j+1 / 2}}{\partial t}=-\frac{\bar{P}_{i+1 / 2, j+1 / 2}-\bar{P}_{i-1 / 2, j+1 / 2}}{d}+f \bar{v}_{i, j+1 / 2} \\
& \frac{\partial v_{i+1 / 2, j}}{\partial t}=-\frac{\bar{P}_{i+1 / 2, j+1 / 2}-\bar{P}_{i+1 / 2, j-1 / 2}}{d}-f \bar{u}_{i+1 / 2, j}
\end{aligned}
$$

where

$$
\begin{aligned}
& \bar{P}_{i+1 / 2, j+1 / 2} \equiv \frac{1}{4}\left(P_{i+1, j+1}+P_{i+1, j-1}+P_{i-1, j+1}+P_{i-1, j-1}\right) \\
& \bar{v}_{i, j+1 / 2} \equiv \frac{1}{4}\left(v_{i-1 / 2, j}+v_{i+1 / 2, j}+v_{i-1 / 2, j+1}+v_{i+1 / 2, j+1}\right)
\end{aligned}
$$


$\bar{u}_{i+1 / 2, j} \equiv \frac{1}{4}\left(u_{i, j+1 / 2}+u_{i, j-1 / 2}+u_{i+1, j+1 / 2}+u_{i+1, j-1 / 2}\right)$

To derive divergence equation for corner points $(i+1 / 2, j+1 / 2)$ :

$$
\begin{aligned}
& \frac{\partial}{\partial t}\left(\frac{u_{i+1, j+1 / 2}-u_{i, j+1 / 2}}{d}\right)=-\frac{\bar{P}_{i+3 / 2, j+1 / 2}-2 \bar{P}_{i+1 / 2, j+1 / 2}+\bar{P}_{i-1 / 2, j+1 / 2}}{d^{2}}+f\left(\frac{\bar{v}_{i+1, j+1 / 2}-\bar{v}_{i, j+1 / 2}}{d}\right) \\
& \frac{\partial}{\partial t}\left(\frac{v_{i+1 / 2, j+1}-v_{i+1 / 2, j}}{d}\right)=-\frac{\bar{P}_{i+1 / 2, j+3 / 2}-2 \bar{P}_{i+1 / 2, j+1 / 2}+\bar{P}_{i+1 / 2, j-1 / 2}}{d^{2}}-f\left(\frac{\bar{u}_{i+1 / 2, j+1}-\bar{u}_{i+1 / 2, j}}{d}\right)
\end{aligned}
$$

Define:

$$
D_{i+1 / 2, j+1 / 2} \equiv \frac{1}{d}\left(u_{i+1, j+1 / 2}-u_{i, j+1 / 2}+v_{i+1 / 2, j+1}-v_{i+1 / 2, j}\right)
$$

Divergence equation (1):

$$
\begin{aligned}
\frac{\partial}{\partial t} D_{i+1 / 2, j+1 / 2}=-\frac{\bar{P}_{i+3 / 2, j+1 / 2}+\bar{P}_{i-1 / 2, j+1 / 2}-}{4} \bar{P}_{i+1 / 2, j+1 / 2}+\bar{P}_{i+1 / 2, j+3 / 2}+\bar{P}_{i+1 / 2, j-1 / 2} & d^{2} \\
+ & f\left(\frac{\bar{v}_{i+1, j+1 / 2}-\bar{v}_{i, j+1 / 2}}{d}-\frac{\bar{u}_{i+1 / 2, j+1}-\bar{u}_{i+1 / 2, j}}{d}\right)
\end{aligned}
$$

Define:

$$
\left(\omega_{z}\right)_{i, j} \equiv \frac{1}{d}\left(v_{i+1 / 2, j}-v_{i-1 / 2, j}-u_{i, j+1 / 2}+u_{i, j-1 / 2}\right)
$$

Divergence equation (2):

Consider (M.6) and (M.7): 


$$
\begin{aligned}
\frac{\partial}{\partial t} D_{i+1 / 2, j+1 / 2}=-\frac{\bar{P}_{i+3 / 2, j+1 / 2}+\bar{P}_{i-1 / 2, j+1 / 2}+}{P_{i+1 / 2, j+3 / 2}+\bar{P}_{i+1 / 2, j-1 / 2}-4 \bar{P}_{i+1 / 2, j+1 / 2}} & d^{2} \\
& +f \frac{1}{4}\left[\left(\omega_{z}\right)_{i+1, j+1}+\left(\omega_{z}\right)_{i-1, j+1}+\left(\omega_{z}\right)_{i+1, j-1}+\left(\omega_{z}\right)_{i, j}\right]
\end{aligned}
$$

To derive vorticity equation for center point $(i, j)$ :

$$
\begin{gathered}
\frac{\partial}{\partial t}\left(\frac{v_{i+1 / 2, j}-v_{i-1 / 2, j}}{d}\right)=-\frac{\bar{P}_{i+1 / 2, j+1 / 2}-\bar{P}_{i+1 / 2, j-1 / 2}-\bar{P}_{i-1 / 2, j+1 / 2}+\bar{P}_{i-1 / 2, j-1 / 2}}{d^{2}}-f\left(\frac{\bar{u}_{i+1 / 2, j}-\bar{u}_{i-1 / 2, j}}{d}\right) \\
\frac{\partial}{\partial t}\left(\frac{-u_{i, j+1 / 2}+u_{i, j-1 / 2}}{d}\right)=\frac{\bar{P}_{i+1 / 2, j+1 / 2}-\bar{P}_{i-1 / 2, j+1 / 2}-\bar{P}_{i+1 / 2, j-1 / 2}+\bar{P}_{i-1 / 2, j-1 / 2}}{d^{2}}-f\left(\frac{\bar{v}_{i, j+1 / 2}-\bar{v}_{i, j-1 / 2}}{d}\right)
\end{gathered}
$$

The vorticity equation (1):

$$
\frac{\partial}{\partial t}\left(\frac{v_{i+1 / 2, j}-v_{i-1 / 2, j}-u_{i, j+1 / 2}+u_{i, j-1 / 2}}{d}\right)=-f\left(\frac{\bar{u}_{i+1 / 2, j}-\bar{u}_{i-1 / 2, j}}{d}+\frac{\bar{v}_{i, j+1 / 2}-\bar{v}_{i, j-1 / 2}}{d}\right)
$$

The vorticity equation (2):

Using (M.7) and (M.5) in (M.11):

$$
\frac{\partial}{\partial t}\left(\omega_{z}\right)_{i, j}=-f \frac{1}{4}\left(D_{i+1 / 2, j+1 / 2}+D_{i-1 / 2, j+1 / 2}+D_{i+1 / 2, j-1 / 2}+D_{i-1 / 2, j-1 / 2}\right)
$$

Vorticity equation from (M.12):

$$
\frac{\partial\left(\omega_{z}\right)_{i, j}}{\partial t}=-f \frac{1}{4}\left(D_{i-1 / 2, j-1 / 2}+D_{i+1 / 2, j-1 / 2}+D_{i-1 / 2, j+1 / 2}+D_{i+1 / 2, j+1 / 2}\right)
$$

Divergence equation from (M.8):

$$
\begin{aligned}
\frac{\partial}{\partial t} D_{i+1 / 2, j+1 / 2} & =f \frac{1}{4}\left[\left(\omega_{z}\right)_{i+1, j+1}+\left(\omega_{z}\right)_{i, j+1}+\left(\omega_{z}\right)_{i+1, j}+\left(\omega_{z}\right)_{i, j}\right] \\
& -\frac{\bar{P}_{i+3 / 2, j+1 / 2}-2 \bar{P}_{i+1 / 2, j+1 / 2}+\bar{P}_{i-1 / 2, j+1 / 2}}{d^{2}}-\frac{\bar{P}_{i+1 / 2, j+3 / 2}-2 \bar{P}_{i+1 / 2, j+1 / 2}+\bar{P}_{i+1 / 2, j-1 / 2}}{d^{2}}
\end{aligned}
$$


where

$$
\bar{P}_{i+1 / 2, j+1 / 2} \equiv \frac{1}{4}\left(P_{i+1, j+1}+P_{i+1, j-1}+P_{i-1, j+1}+P_{i-1, j-1}\right)
$$

Vertical momentum equation:

$$
\frac{\partial w_{i, j}}{\partial t}=-\left[\frac{\partial}{\partial z}-\left(\frac{1}{2 \rho_{0}} \frac{\partial \rho_{0}}{\partial z}\right)\right] P_{i, j}+B_{i, j}
$$

Thermodynamic equation:

$$
\frac{\partial B_{i, j}}{\partial t}=-N^{2} w_{i, j}
$$

Mass continuity equation:

$$
\frac{1}{4}\left(D_{i-1 / 2, j-1 / 2}+D_{i+1 / 2, j-1 / 2}+D_{i-1 / 2, j+1 / 2}+D_{i+1 / 2, j+1 / 2}\right)+\left[\frac{\partial}{\partial z}+\left(\frac{1}{2 \rho_{0}} \frac{\partial \rho_{0}}{\partial z}\right)\right] w_{i, j}=0
$$

From (4)-(6):

$$
\left(\frac{\partial^{2}}{\partial t^{2}}+N^{2}\right)\left[\frac{1}{4}\left(D_{i-1 / 2, j-1 / 2}+D_{i+1 / 2, j-1 / 2}+D_{i-1 / 2, j+1 / 2}+D_{i+1 / 2, j+1 / 2}\right)\right]-\left[\frac{\partial^{2}}{\partial z^{2}}-\left(\frac{1}{2 \rho_{0}} \frac{\partial \rho_{0}}{\partial z}\right)^{2}\right] \frac{\partial}{\partial t} P_{i, j}=0
$$

Define:

$\phi_{i, j}(z, t) \equiv \operatorname{Re}\left\{\hat{\Phi} e^{i(k d i+\ell d j+m z-v t)}\right\} \quad$ and $\quad \phi_{i+1 / 2, j+1 / 2}(z, t) \equiv \operatorname{Re}\left\{\hat{\Phi} e^{i[k d(i+1 / 2)+\ell d(j+1 / 2)+m z-v t]}\right\}$

Using (8) in (1): 


$$
-i v \hat{\omega}_{z}=-f \frac{1}{4}\left(e^{-i k \frac{d}{2}} e^{-i \ell \frac{d}{2}}+e^{i k \frac{d}{2}} e^{-i \ell \frac{d}{2}}+e^{-i k \frac{d}{2}} e^{i \ell \frac{d}{2}}+e^{i k \frac{d}{2}} e^{i \ell \frac{d}{2}}\right) \hat{D}
$$

Using (8) in (2):

$$
\begin{aligned}
-\underset{\sim}{i v} \hat{D} & =f \frac{1}{4}\left(e^{i k \frac{d}{2}} e^{i \ell \frac{d}{2}}+e^{-i k \frac{d}{2}} e^{i \ell \frac{d}{2}}+e^{i k \frac{d}{2}} e^{-i \ell \frac{d}{2}}+e^{-i k \frac{d}{2}} e^{-i \ell \frac{d}{2}}\right) \hat{\omega}_{z} \\
& -\frac{1}{4}\left(e^{-i k \frac{d}{2}} e^{-i \ell \frac{d}{2}}+e^{i k \frac{d}{2}} e^{-i \ell \frac{d}{2}}+e^{-i k \frac{d}{2}} e^{i \ell \frac{d}{2}}+e^{i k \frac{d}{2}} e^{i \ell \frac{d}{2}}\right)\left(\frac{e^{i k d}+e^{-i k d}+e^{i \ell d}+e^{-i \ell d}-4}{d^{2}}\right) \hat{P}
\end{aligned}
$$

Using (8) in (7):

$$
\left(-v^{2}+N^{2}\right)\left[\frac{1}{4}\left(e^{i k \frac{d}{2}} e^{i \ell \frac{d}{2}}+e^{-i k \frac{d}{2}} e^{i \ell \frac{d}{2}}+e^{i k \frac{d}{2}} e^{-i \ell \frac{d}{2}}+e^{-i k \frac{d}{2}} e^{-i \ell \frac{d}{2}}\right)\right] \hat{D}-\left[m^{2}+\left(\frac{1}{2 \rho_{0}} \frac{\partial \rho_{0}}{\partial z}\right)^{2}\right] i v P_{i, j}=0
$$

$\ln (9)-(11)$ :

$$
\begin{aligned}
\mu & \equiv \frac{1}{4}\left(e^{-i k \frac{d}{2}} e^{-i \ell \frac{d}{2}}+e^{i k \frac{d}{2}} e^{-i \ell \frac{d}{2}}+e^{-i k \frac{d}{2}} e^{i \ell \frac{d}{2}}+e^{i k \frac{d}{2}} e^{i \ell \frac{d}{2}}\right) \\
& =\frac{1}{4}\left[\left(e^{-i k \frac{d}{2}}+e^{i k \frac{d}{2}}\right)\left(e^{-i \ell \frac{d}{2}}+e^{i \ell \frac{d}{2}}\right)\right]=\cos \left(k \frac{d}{2}\right) \cos \left(\ell \frac{d}{2}\right)
\end{aligned}
$$

$\ln (10)$ :

$$
\begin{aligned}
\frac{e^{i k d}+e^{-i k d}+e^{i \ell d}+e^{-i \ell d}-4}{d^{2}} & =\frac{2 \cos (k d)+2 \cos (\ell d)-4}{d^{2}} \\
= & \left(\frac{-\sin ^{2}\left(\frac{1}{2} k d\right)}{\left(\frac{1}{2} k d\right)^{2}} k^{2}+\frac{-\sin ^{2}\left(\frac{1}{2} \ell d\right)}{\left(\frac{1}{2} \ell d\right)^{2}} \ell^{2}\right)
\end{aligned}
$$

Rewrite (13):

$$
\frac{e^{i k d}+e^{-i k d}+e^{i \ell d}+e^{-i \ell d}-4}{d^{2}}=-\xi^{2} k^{2}-\eta^{2} \ell^{2}
$$

where

$$
\xi \equiv \frac{\sin \left(\frac{1}{2} k d\right)}{\frac{1}{2} k d} \quad \text { and } \quad \eta \equiv \frac{\sin \left(\frac{1}{2} \ell d\right)}{\frac{1}{2} \ell d}
$$


Using (12) and (14a) in (9)-(11):

$$
\begin{gathered}
-i v \hat{\omega}_{z}=-\mu f \hat{D} \\
-i v \hat{D}=\mu\left(\xi^{2} k^{2}+\eta^{2} \ell^{2}\right) \hat{P}+\mu f \hat{\omega}_{z} \\
\mu\left(-v^{2}+N^{2}\right) \hat{D}+\left[\frac{\partial^{2}}{\partial z^{2}}-\left(\frac{1}{2 \rho_{0}} \frac{\partial \rho_{0}}{\partial z}\right)^{2}\right] i v \hat{P}=0
\end{gathered}
$$

From (20) and (21):

$$
\left(v^{2}-\mu^{2} f^{2}\right) \hat{D}=\mu\left(\xi^{2} k^{2}+\eta^{2} \ell^{2}\right) i v \hat{P}
$$

From (22) and (23):

$$
v^{2}=\frac{\mu^{2} N^{2}\left(\xi^{2} k^{2}+\eta^{2} \ell^{2}\right)+\mu^{2} f^{2}\left(m^{2}+\frac{1}{4 H^{2}}\right)}{\mu^{2}\left(\xi^{2} k^{2}+\eta^{2} \ell^{2}\right)+\left(m^{2}+\frac{1}{4 H^{2}}\right)}
$$

\section{Rossby modes}

Baroclinic modes:

Adding the beta effect to the vorticity equation (1):

$$
\frac{\partial\left(\omega_{z}\right)_{i, j}}{\partial t}=-f_{0} \frac{1}{4}\left(D_{i-1 / 2, j-1 / 2}+D_{i+1 / 2, j-1 / 2}+D_{i-1 / 2, j+1 / 2}+D_{i+1 / 2, j+1 / 2}\right)-\frac{\beta}{f_{0}} \frac{P_{i+1, j}-P_{i-1, j}}{2 d}
$$

Dropping $\partial D / \partial t$ in (2): 


$$
\begin{aligned}
& 0=-\frac{\bar{P}_{i+3 / 2, j+1 / 2}+\bar{P}_{i-1 / 2, j+1 / 2}+\bar{P}_{i+1 / 2, j+3 / 2}+\bar{P}_{i+1 / 2, j-1 / 2}-4 \bar{P}_{i+1 / 2, j+1 / 2}}{d^{2}} \\
&+f_{0} \frac{1}{4}\left[\left(\omega_{z}\right)_{i+1, j+1}+\left(\omega_{z}\right)_{i, j+1}+\left(\omega_{z}\right)_{i+1, j}+\left(\omega_{z}\right)_{i, j}\right]
\end{aligned}
$$

Dropping $\partial^{2} D / \partial t^{2}$ in (7):

$$
N^{2}\left[\frac{1}{4}\left(D_{i-1 / 2, j-1 / 2}+D_{i+1 / 2, j-1 / 2}+D_{i-1 / 2, j+1 / 2}+D_{i+1 / 2, j+1 / 2}\right)\right]-\left[\frac{\partial^{2}}{\partial z^{2}}-\left(\frac{1}{2 \rho_{0}} \frac{\partial \rho_{0}}{\partial z}\right)^{2}\right] \frac{\partial}{\partial t} P_{i, j}=0
$$

From (30)-(32):

$$
\begin{gathered}
-\underset{\sim}{i} v \hat{\omega}_{z}=-\mu f_{0} \hat{D}-\frac{\beta}{f_{0}} \frac{e^{i k d}-e^{-i k d}}{2 d} \hat{P} \\
0=\mu\left(\xi^{2} k^{2}+\eta^{2} \ell^{2}\right) \hat{P}+\mu f_{0} \hat{\omega}_{z} \\
\mu \hat{D} N^{2}-\left[m^{2}+\left(\frac{1}{2 \rho_{0}} \frac{\partial \rho_{0}}{\partial z}\right)^{2}\right] \underset{\sim}{i} v \hat{P}=0
\end{gathered}
$$

Rewrite (40):

$$
-\underset{\sim}{i} v \hat{\omega}_{z}=-\mu f_{0} \hat{D}-\underset{\sim}{i} \frac{\beta}{f_{0}} \frac{2 \sin (k d)}{2 d} \hat{P}
$$

Rewrite (43):

$$
-\underset{\sim}{v} v \hat{\omega}_{z}=-\mu f_{0} \hat{D}-\underset{\sim}{i} \frac{\beta}{f_{0}} \tilde{\xi}_{k} \hat{P}
$$

where

$$
\tilde{\xi} \equiv \frac{\sin (k d)}{k d}
$$

From (41), (42) and (44): 


$$
v N^{2}\left(\xi^{2} k^{2}+\eta^{2} \ell^{2}\right) \hat{P}=-f_{0}^{2}\left[m^{2}+\left(\frac{1}{2 \rho_{0}} \frac{\partial \rho_{0}}{\partial z}\right)^{2}\right] v \hat{P}-N^{2} \beta \tilde{\xi} k \hat{P}
$$

Using $\frac{1}{\rho_{0}} \frac{\partial \rho_{0}}{\partial z}=-\frac{1}{H}$ in (46) and for nontrivial solutions $(\hat{P} \neq 0)$ :

$$
v=\frac{-\beta \tilde{\xi}_{k}}{\left(\xi^{2} k^{2}+\eta^{2} \ell^{2}\right)+\frac{f_{0}^{2}}{N^{2}}\left(m^{2}+\frac{1}{4 H}\right)}
$$

Barotropic modes:

Assuming $D=0$ in (30)

$$
\frac{\partial\left(\omega_{z}\right)_{i, j}}{\partial t}=-\frac{\beta}{f_{0}} \frac{P_{i+1, j}-P_{i-1, j}}{2 d}
$$

Writing (31) again:

$$
\begin{aligned}
0=-\frac{\bar{P}_{i+3 / 2, j+1 / 2}+\bar{P}_{i-1 / 2, j+1 / 2}+\bar{P}_{i+1 / 2, j+3 / 2}+\bar{P}_{i+1 / 2, j-1 / 2}-4 \bar{P}_{i+1 / 2, j+1 / 2}}{d^{2}} & \\
& +f_{0} \frac{1}{4}\left[\left(\omega_{z}\right)_{i+1, j+1}+\left(\omega_{z}\right)_{i, j+1}+\left(\omega_{z}\right)_{i+1, j}+\left(\omega_{z}\right)_{i, j}\right]
\end{aligned}
$$

Assuming $D=0$ in (32)

$$
-\left[\frac{\partial^{2}}{\partial z^{2}}-\left(\frac{1}{2 \rho_{0}} \frac{\partial \rho_{0}}{\partial z}\right)^{2}\right] \frac{\partial}{\partial t} P_{i, j}=0
$$

( $P$ is vertically constant).

From (50) and (51):

$$
0=v\left(\xi^{2} k^{2}+\eta^{2} \ell^{2}\right) \hat{P}+\beta \tilde{\xi}_{k} \hat{P}
$$

From (53) and for nontrivial solutions $(\hat{P} \neq 0)$ : 


$$
v=\frac{-\beta \tilde{\xi} k}{\xi^{2} k^{2}+\eta^{2} \ell^{2}}
$$




\section{D-grid (with w at corner points)}

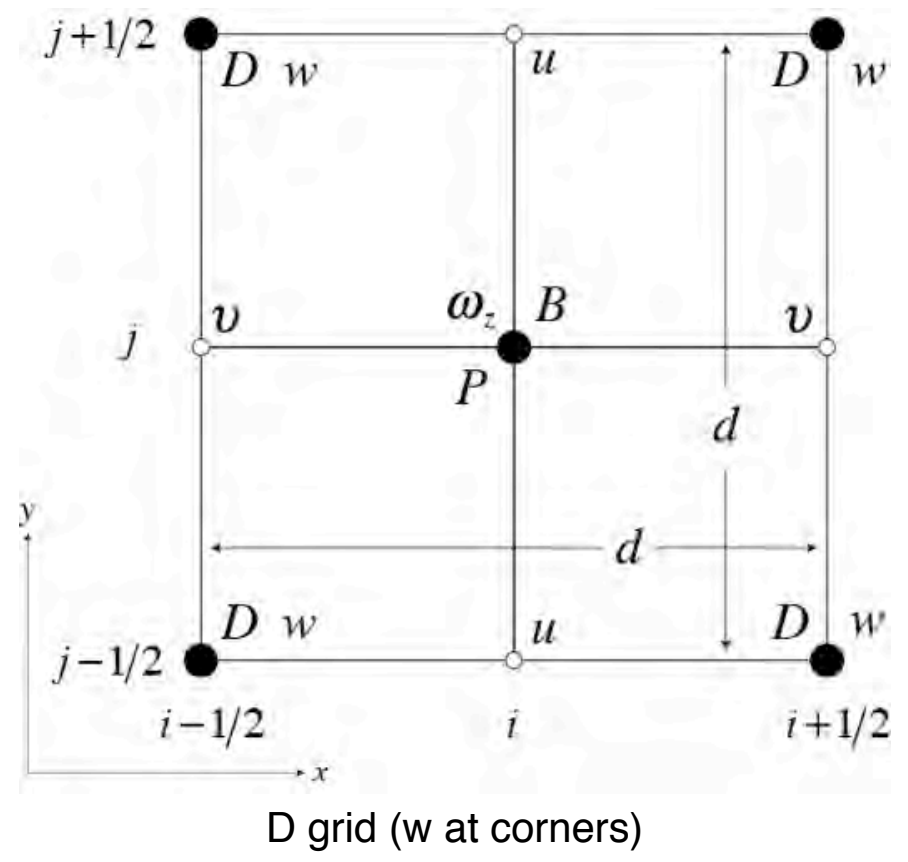

\section{Inertia-gravity modes}

Horizontal momentum equation:

$$
\begin{aligned}
& \frac{\partial u_{i, j+1 / 2}}{\partial t}=-\frac{\bar{P}_{i+1 / 2, j+1 / 2}-\bar{P}_{i-1 / 2, j+1 / 2}}{d}+f \bar{v}_{i, j+1 / 2} \\
& \frac{\partial v_{i+1 / 2, j}}{\partial t}=-\frac{\bar{P}_{i+1 / 2, j+1 / 2}-\bar{P}_{i+1 / 2, j-1 / 2}}{d}-f \bar{u}_{i+1 / 2, j}
\end{aligned}
$$

where

$$
\begin{aligned}
& \bar{P}_{i+1 / 2, j+1 / 2} \equiv \frac{1}{4}\left(P_{i+1, j+1}+P_{i+1, j-1}+P_{i-1, j+1}+P_{i-1, j-1}\right) \\
& \bar{v}_{i, j+1 / 2} \equiv \frac{1}{4}\left(v_{i-1 / 2, j}+v_{i+1 / 2, j}+v_{i-1 / 2, j+1}+v_{i+1 / 2, j+1}\right)
\end{aligned}
$$


$\bar{u}_{i+1 / 2, j} \equiv \frac{1}{4}\left(u_{i, j+1 / 2}+u_{i, j-1 / 2}+u_{i+1, j+1 / 2}+u_{i+1, j-1 / 2}\right)$

To derive divergence equation for corner points $(i+1 / 2, j+1 / 2)$ :

$$
\begin{aligned}
\frac{\partial}{\partial t}\left(\frac{u_{i+1, j+1 / 2}-u_{i, j+1 / 2}}{d}\right) & =-\frac{\bar{P}_{i+3 / 2, j+1 / 2}-2 \bar{P}_{i+1 / 2, j+1 / 2}+\bar{P}_{i-1 / 2, j+1 / 2}}{d^{2}}+f\left(\frac{\bar{v}_{i+1, j+1 / 2}-\bar{v}_{i, j+1 / 2}}{d}\right) \\
\frac{\partial}{\partial t}\left(\frac{v_{i+1 / 2, j+1}-v_{i+1 / 2, j}}{d}\right) & =-\frac{\bar{P}_{i+1 / 2, j+3 / 2}-2 \bar{P}_{i+1 / 2, j+1 / 2}+\bar{P}_{i+1 / 2, j-1 / 2}}{d^{2}}-f\left(\frac{\bar{u}_{i+1 / 2, j+1}-\bar{u}_{i+1 / 2, j}}{d}\right)
\end{aligned}
$$

Define:

$$
D_{i+1 / 2, j+1 / 2} \equiv \frac{1}{d}\left(u_{i+1, j+1 / 2}-u_{i, j+1 / 2}+v_{i+1 / 2, j+1}-v_{i+1 / 2, j}\right)
$$

Divergence equation (1):

$$
\begin{aligned}
& \frac{\partial}{\partial t} D_{i+1 / 2, j+1 / 2}=-\frac{\bar{P}_{i+3 / 2, j+1 / 2}+\bar{P}_{i-1 / 2, j+1 / 2}-}{} 4 \bar{P}_{i+1 / 2, j+1 / 2}+\bar{P}_{i+1 / 2, j+3 / 2}+\bar{P}_{i+1 / 2, j-1 / 2} \\
& d^{2} \\
&+f\left(\frac{\bar{v}_{i+1, j+1 / 2}-\bar{v}_{i, j+1 / 2}}{d}-\frac{\bar{u}_{i+1 / 2, j+1}-\bar{u}_{i+1 / 2, j}}{d}\right)
\end{aligned}
$$

Define:

$$
\left(\omega_{z}\right)_{i, j} \equiv \frac{1}{d}\left(v_{i+1 / 2, j}-v_{i-1 / 2, j}-u_{i, j+1 / 2}+u_{i, j-1 / 2}\right)
$$

Divergence equation (2):

Consider (M.6) and (M.7): 


$$
\begin{aligned}
\frac{\partial}{\partial t} D_{i+1 / 2, j+1 / 2}=-\frac{\bar{P}_{i+3 / 2, j+1 / 2}+\bar{P}_{i-1 / 2, j+1 / 2}+}{P_{i+1 / 2, j+3 / 2}+\bar{P}_{i+1 / 2, j-1 / 2}-4 \bar{P}_{i+1 / 2, j+1 / 2}} & d^{2} \\
& +f \frac{1}{4}\left[\left(\omega_{z}\right)_{i+1, j+1}+\left(\omega_{z}\right)_{i-1, j+1}+\left(\omega_{z}\right)_{i+1, j-1}+\left(\omega_{z}\right)_{i, j}\right]
\end{aligned}
$$

To derive vorticity equation for center point $(i, j)$ :

$$
\begin{gathered}
\frac{\partial}{\partial t}\left(\frac{v_{i+1 / 2, j}-v_{i-1 / 2, j}}{d}\right)=-\frac{\bar{P}_{i+1 / 2, j+1 / 2}-\bar{P}_{i+1 / 2, j-1 / 2}-\bar{P}_{i-1 / 2, j+1 / 2}+\bar{P}_{i-1 / 2, j-1 / 2}}{d^{2}}-f\left(\frac{\bar{u}_{i+1 / 2, j}-\bar{u}_{i-1 / 2, j}}{d}\right) \\
\frac{\partial}{\partial t}\left(\frac{-u_{i, j+1 / 2}+u_{i, j-1 / 2}}{d}\right)=\frac{\bar{P}_{i+1 / 2, j+1 / 2}-\bar{P}_{i-1 / 2, j+1 / 2}-\bar{P}_{i+1 / 2, j-1 / 2}+\bar{P}_{i-1 / 2, j-1 / 2}}{d^{2}}-f\left(\frac{\bar{v}_{i, j+1 / 2}-\bar{v}_{i, j-1 / 2}}{d}\right)
\end{gathered}
$$

The vorticity equation (1):

$$
\frac{\partial}{\partial t}\left(\frac{v_{i+1 / 2, j}-v_{i-1 / 2, j}-u_{i, j+1 / 2}+u_{i, j-1 / 2}}{d}\right)=-f\left(\frac{\bar{u}_{i+1 / 2, j}-\bar{u}_{i-1 / 2, j}}{d}+\frac{\bar{v}_{i, j+1 / 2}-\bar{v}_{i, j-1 / 2}}{d}\right)
$$

The vorticity equation (2):

Using (M.7) and (M.5) in (M.11):

$$
\frac{\partial}{\partial t}\left(\omega_{z}\right)_{i, j}=-f \frac{1}{4}\left(D_{i+1 / 2, j+1 / 2}+D_{i-1 / 2, j+1 / 2}+D_{i+1 / 2, j-1 / 2}+D_{i-1 / 2, j-1 / 2}\right)
$$

Vorticity equation from (M.12):

$$
\frac{\partial\left(\omega_{z}\right)_{i, j}}{\partial t}=-f \frac{1}{4}\left(D_{i-1 / 2, j-1 / 2}+D_{i+1 / 2, j-1 / 2}+D_{i-1 / 2, j+1 / 2}+D_{i+1 / 2, j+1 / 2}\right)
$$

Divergence equation from (M.8):

$$
\begin{aligned}
\frac{\partial}{\partial t} D_{i+1 / 2, j+1 / 2} & =f \frac{1}{4}\left[\left(\omega_{z}\right)_{i+1, j+1}+\left(\omega_{z}\right)_{i, j+1}+\left(\omega_{z}\right)_{i+1, j}+\left(\omega_{z}\right)_{i, j}\right] \\
& -\frac{\bar{P}_{i+3 / 2, j+1 / 2}-2 \bar{P}_{i+1 / 2, j+1 / 2}+\bar{P}_{i-1 / 2, j+1 / 2}}{d^{2}}-\frac{\bar{P}_{i+1 / 2, j+3 / 2}-2 \bar{P}_{i+1 / 2, j+1 / 2}+\bar{P}_{i+1 / 2, j-1 / 2}}{d^{2}}
\end{aligned}
$$


where

$$
\bar{P}_{i+1 / 2, j+1 / 2} \equiv \frac{1}{4}\left(P_{i+1, j+1}+P_{i+1, j-1}+P_{i-1, j+1}+P_{i-1, j-1}\right)
$$

Vertical momentum equation:

$$
\begin{array}{r}
\frac{\partial w_{i+1 / 2, j+1 / 2}}{\partial t}=-\left[\frac{\partial}{\partial z}-\left(\frac{1}{2 \rho_{0}} \frac{\partial \rho_{0}}{\partial z}\right)\right] \frac{1}{4}\left(P_{i+1, j+1}+P_{i-1, j+1}+P_{i+1, j-1}+P_{i, j}\right) \\
+\frac{1}{4}\left(B_{i+1, j+1}+B_{i-1, j+1}+B_{i+1, j-1}+B_{i, j}\right)
\end{array}
$$

Thermodynamic equation:

$$
\frac{\partial B_{i, j}}{\partial t}=-N^{2} \frac{1}{4}\left(w_{i+1 / 2, j+1 / 2}+w_{i-1 / 2, j+1 / 2}+w_{i+1 / 2, j-1 / 2}+w_{i-1 / 2, j-1 / 2}\right)
$$

Mass continuity equation:

$$
D_{i+1 / 2, j+1 / 2}+\left[\frac{\partial}{\partial z}+\left(\frac{1}{2 \rho_{0}} \frac{\partial \rho_{0}}{\partial z}\right)\right] w_{i+1 / 2, j+1 / 2}=0
$$

From (4)-(6):

$$
\begin{aligned}
& -\frac{\partial D_{i+1 / 2, j+1 / 2}}{\partial t}=-\left[\frac{\partial^{2}}{\partial z^{2}}-\left(\frac{1}{2 \rho_{0}} \frac{\partial \rho_{0}}{\partial z}\right)^{2}\right] \frac{1}{4}\left(P_{i+1, j+1}+P_{i-1, j+1}+P_{i+1, j-1}+P_{i, j}\right) \\
& +\frac{1}{4}\left[\frac{\partial}{\partial z}+\left(\frac{1}{2 \rho_{0}} \frac{\partial \rho_{0}}{\partial z}\right)\right]\left(B_{i+1, j+1}+B_{i-1, j+1}+B_{i+1, j-1}+B_{i, j}\right) \\
& {\left[\frac{\partial}{\partial z}+\left(\frac{1}{2 \rho_{0}} \frac{\partial \rho_{0}}{\partial z}\right)\right] \frac{\partial B_{i, j}}{\partial t}=N^{2} \frac{1}{4}\left(D_{i+1 / 2, j+1 / 2}+D_{i-1 / 2, j+1 / 2}+D_{i+1 / 2, j-1 / 2}+D_{i-1 / 2, j-1 / 2}\right)}
\end{aligned}
$$


Using (12) and (14a) in (9)-(11):

$$
\begin{gathered}
-i v \hat{\omega}_{z}=-\mu f \hat{D} \\
-i v \hat{D}=\mu\left(\xi^{2} k^{2}+\eta^{2} \ell^{2}\right) \hat{P}+\mu f \hat{\omega}_{z} \\
\left(v^{2}-\mu^{2} N^{2}\right) \hat{D}=\mu\left[\frac{\partial^{2}}{\partial z^{2}}-\left(\frac{1}{2 \rho_{0}} \frac{\partial \rho_{0}}{\partial z}\right)^{2}\right] \underset{j}{i} \hat{P}
\end{gathered}
$$

From (20) and (21):

$$
\left(v^{2}-\mu^{2} f^{2}\right) \hat{D}=\mu\left(\xi^{2} k^{2}+\eta^{2} \ell^{2}\right) i v \hat{P}
$$

From (22) and (23):

$$
v^{2}=\frac{\mu^{2} N^{2}\left(\xi^{2} k^{2}+\eta^{2} \ell^{2}\right)+\mu^{2} f^{2}\left(m^{2}+\frac{1}{4 H^{2}}\right)}{\left(\xi^{2} k^{2}+\eta^{2} \ell^{2}\right)+\left(m^{2}+\frac{1}{4 H^{2}}\right)}
$$

\section{Rossby modes}

Baroclinic modes:

Adding the beta effect to the vorticity equation (1):

$$
\frac{\partial\left(\omega_{z}\right)_{i, j}}{\partial t}=-f_{0} \frac{1}{4}\left(D_{i-1 / 2, j-1 / 2}+D_{i+1 / 2, j-1 / 2}+D_{i-1 / 2, j+1 / 2}+D_{i+1 / 2, j+1 / 2}\right)-\frac{\beta}{f_{0}} \frac{P_{i+1, j}-P_{i-1, j}}{2 d}
$$

Dropping $\partial D / \partial t$ in (2): 


$$
\begin{aligned}
& 0=-\frac{\bar{P}_{i+3 / 2, j+1 / 2}+\bar{P}_{i-1 / 2, j+1 / 2}+\bar{P}_{i+1 / 2, j+3 / 2}+\bar{P}_{i+1 / 2, j-1 / 2}-4 \bar{P}_{i+1 / 2, j+1 / 2}}{d^{2}} \\
&+f_{0} \frac{1}{4}\left[\left(\omega_{z}\right)_{i+1, j+1}+\left(\omega_{z}\right)_{i, j+1}+\left(\omega_{z}\right)_{i+1, j}+\left(\omega_{z}\right)_{i, j}\right]
\end{aligned}
$$

Dropping $\partial^{2} D / \partial t^{2}$ in (7):

$$
\begin{aligned}
& 0=-\left[\frac{\partial^{2}}{\partial z^{2}}-\left(\frac{1}{2 \rho_{0}} \frac{\partial \rho_{0}}{\partial z}\right)^{2}\right] \frac{1}{4}\left(P_{i+1, j+1}+P_{i-1, j+1}+P_{i+1, j-1}+P_{i, j}\right) \\
& +\frac{1}{4}\left[\frac{\partial}{\partial z}+\left(\frac{1}{2 \rho_{0}} \frac{\partial \rho_{0}}{\partial z}\right)\right]\left(B_{i+1, j+1}+B_{i-1, j+1}+B_{i+1, j-1}+B_{i, j}\right) \\
& {\left[\frac{\partial}{\partial z}+\left(\frac{1}{2 \rho_{0}} \frac{\partial \rho_{0}}{\partial z}\right)\right] \frac{\partial B_{i, j}}{\partial t}=N^{2} \frac{1}{4}\left(D_{i+1 / 2, j+1 / 2}+D_{i-1 / 2, j+1 / 2}+D_{i+1 / 2, j-1 / 2}+D_{i-1 / 2, j-1 / 2}\right)}
\end{aligned}
$$

From (30)-(32):

$$
\begin{gathered}
-\underset{\sim}{i} v \hat{\omega}_{z}=-\mu f_{0} \hat{D}-\frac{\beta}{f_{0}} \frac{e^{i k d}-e^{-i k d}}{2 d} \hat{P} \\
0=\mu\left(\xi^{2} k^{2}+\eta^{2} \ell^{2}\right) \hat{P}+\mu f_{0} \hat{\omega}_{z} \\
\mu \hat{D} N^{2}-\left[m^{2}+\left(\frac{1}{2 \rho_{0}} \frac{\partial \rho_{0}}{\partial z}\right)^{2}\right] \underset{\sim}{i} v \hat{P}=0
\end{gathered}
$$

Rewrite (40):

$$
-\underset{\sim}{v} v \hat{\omega}_{z}=-\mu f_{0} \hat{D}-\underset{\sim}{i} \frac{\beta}{f_{0}} \frac{2 \sin (k d)}{2 d} \hat{P}
$$

Rewrite (43):

$$
-\underset{\sim}{i} v \hat{\omega}_{z}=-\mu f_{0} \hat{D}-\underset{\sim}{i} \frac{\beta}{f_{0}} \tilde{\xi}_{k} \hat{P}
$$

where 


$$
\tilde{\xi} \equiv \frac{\sin (k d)}{k d}
$$

From (41), (42) and (44):

$$
v N^{2}\left(\xi^{2} k^{2}+\eta^{2} \ell^{2}\right) \hat{P}=-f_{0}^{2}\left[m^{2}+\left(\frac{1}{2 \rho_{0}} \frac{\partial \rho_{0}}{\partial z}\right)^{2}\right] v \hat{P}-N^{2} \beta \tilde{\xi}_{k} \hat{P}
$$

Using $\frac{1}{\rho_{0}} \frac{\partial \rho_{0}}{\partial z}=-\frac{1}{H}$ in (46) and for nontrivial solutions $(\hat{P} \neq 0)$ :

$$
v=\frac{-\beta \tilde{\xi}_{k}}{\left(\xi^{2} k^{2}+\eta^{2} \ell^{2}\right)+\frac{f_{0}^{2}}{N^{2}}\left(m^{2}+\frac{1}{4 H}\right)}
$$

Barotropic modes:

Assuming $D=0$ in (30)

$$
\frac{\partial\left(\omega_{z}\right)_{i, j}}{\partial t}=-\frac{\beta}{f_{0}} \frac{P_{i+1, j}-P_{i-1, j}}{2 d}
$$

Writing (31) again:

$$
\begin{aligned}
& 0=-\frac{\bar{P}_{i+3 / 2, j+1 / 2}+\bar{P}_{i-1 / 2, j+1 / 2}+\bar{P}_{i+1 / 2, j+3 / 2}+\bar{P}_{i+1 / 2, j-1 / 2}-4 \bar{P}_{i+1 / 2, j+1 / 2}}{d^{2}} \\
&+f_{0} \frac{1}{4}\left[\left(\omega_{z}\right)_{i+1, j+1}+\left(\omega_{z}\right)_{i, j+1}+\left(\omega_{z}\right)_{i+1, j}+\left(\omega_{z}\right)_{i, j}\right]
\end{aligned}
$$

Assuming $D=0$ in (32)

$$
-\left[\frac{\partial^{2}}{\partial z^{2}}-\left(\frac{1}{2 \rho_{0}} \frac{\partial \rho_{0}}{\partial z}\right)^{2}\right] \frac{\partial}{\partial t} P_{i, j}=0
$$

( $P$ is vertically constant).

From (50) and (51): 


$$
0=v\left(\xi^{2} k^{2}+\eta^{2} \ell^{2}\right) \hat{P}+\beta \tilde{\xi}_{k} \hat{P}
$$

From (53) and for nontrivial solutions $(\hat{P} \neq 0)$ :

$$
v=\frac{-\beta \tilde{\xi}_{k}}{\xi^{2} k^{2}+\eta^{2} \ell^{2}}
$$


(a)

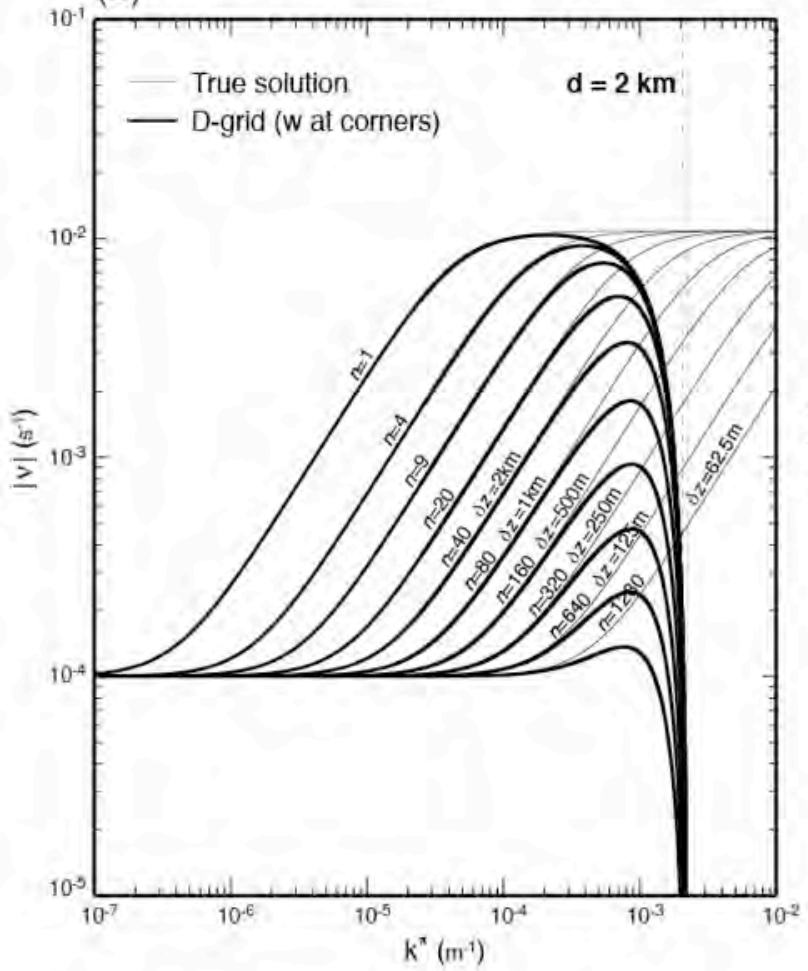

(c)

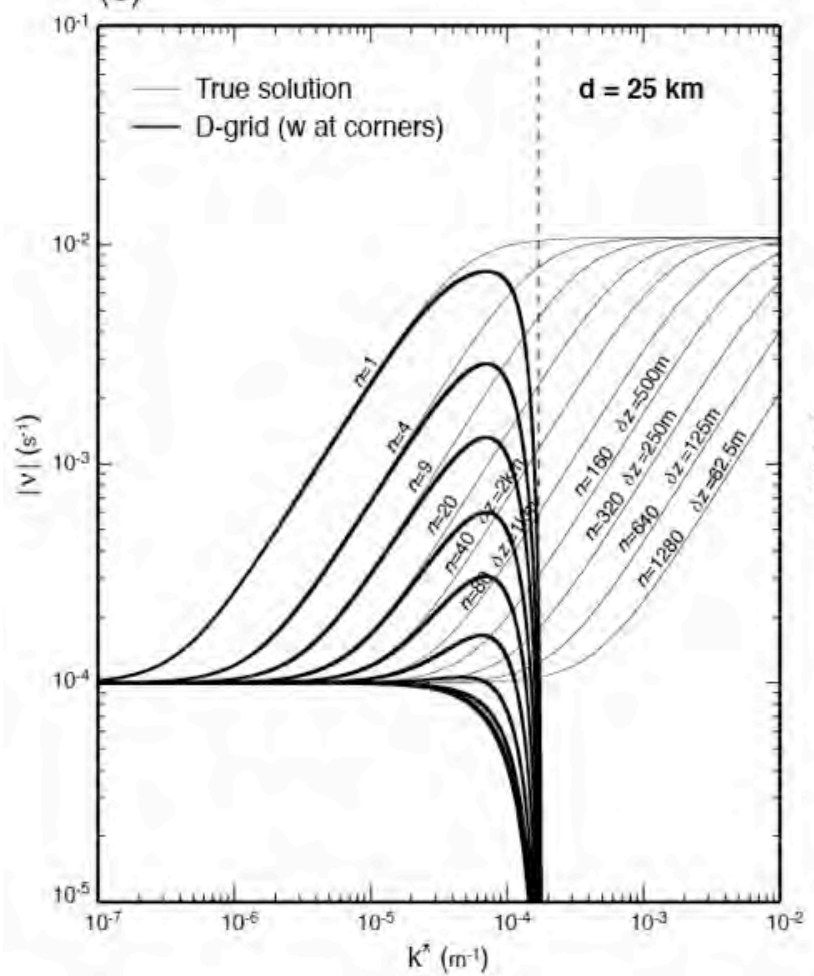

(b)

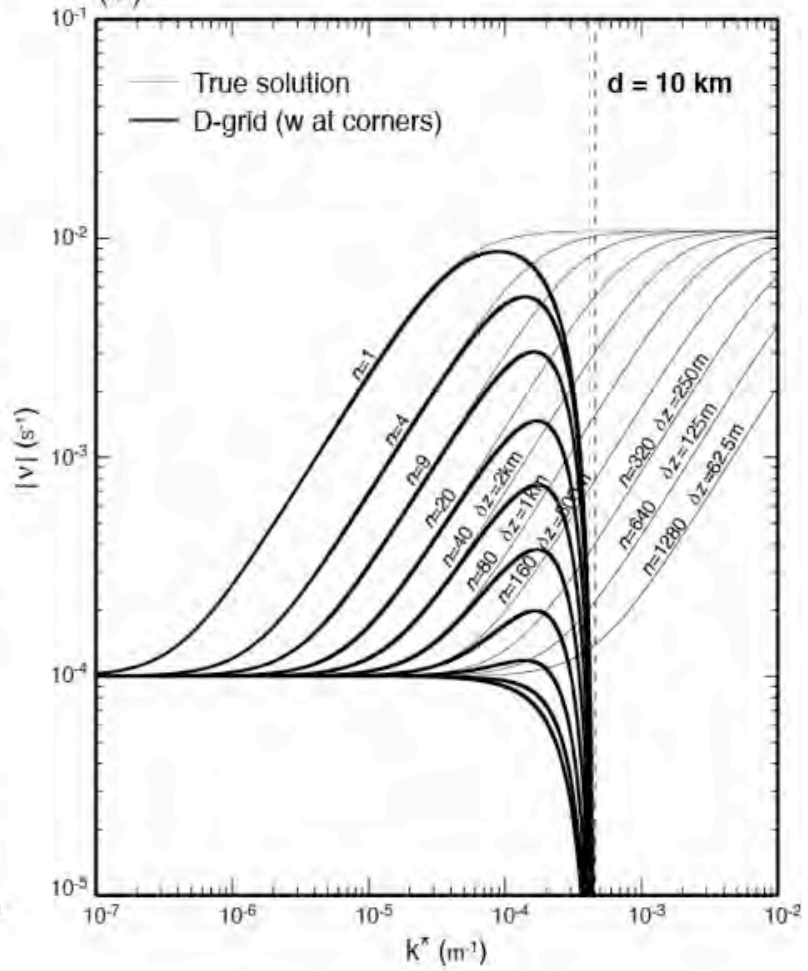

(d)

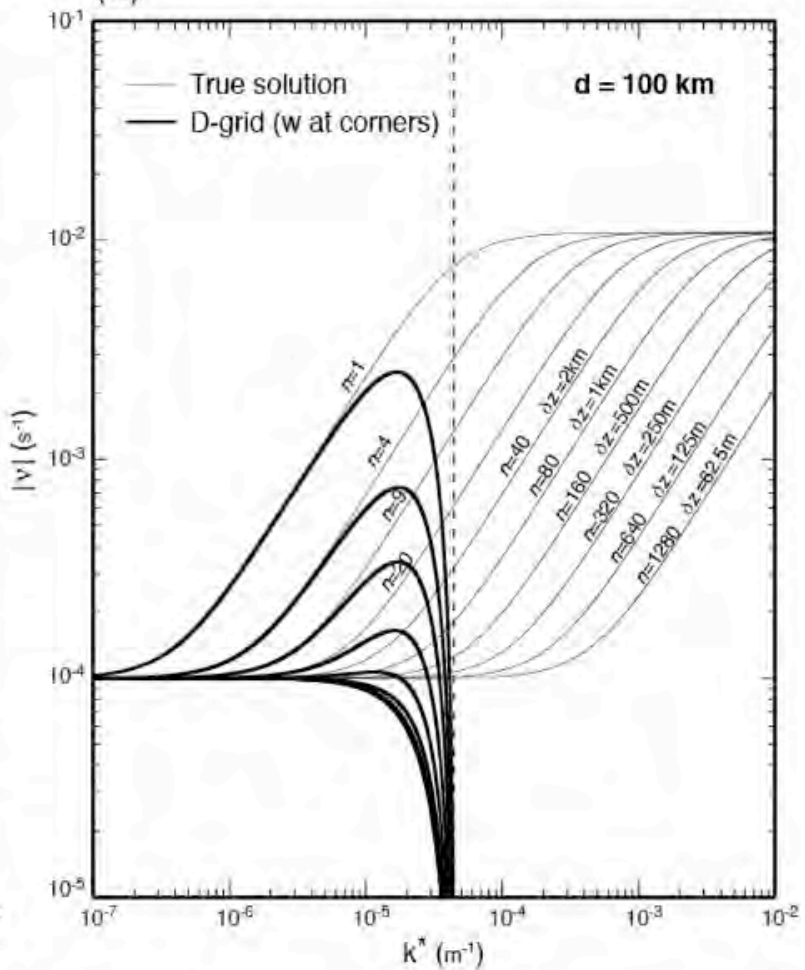

Fig. D-grid (w at the corners). 


\section{CD-grid}

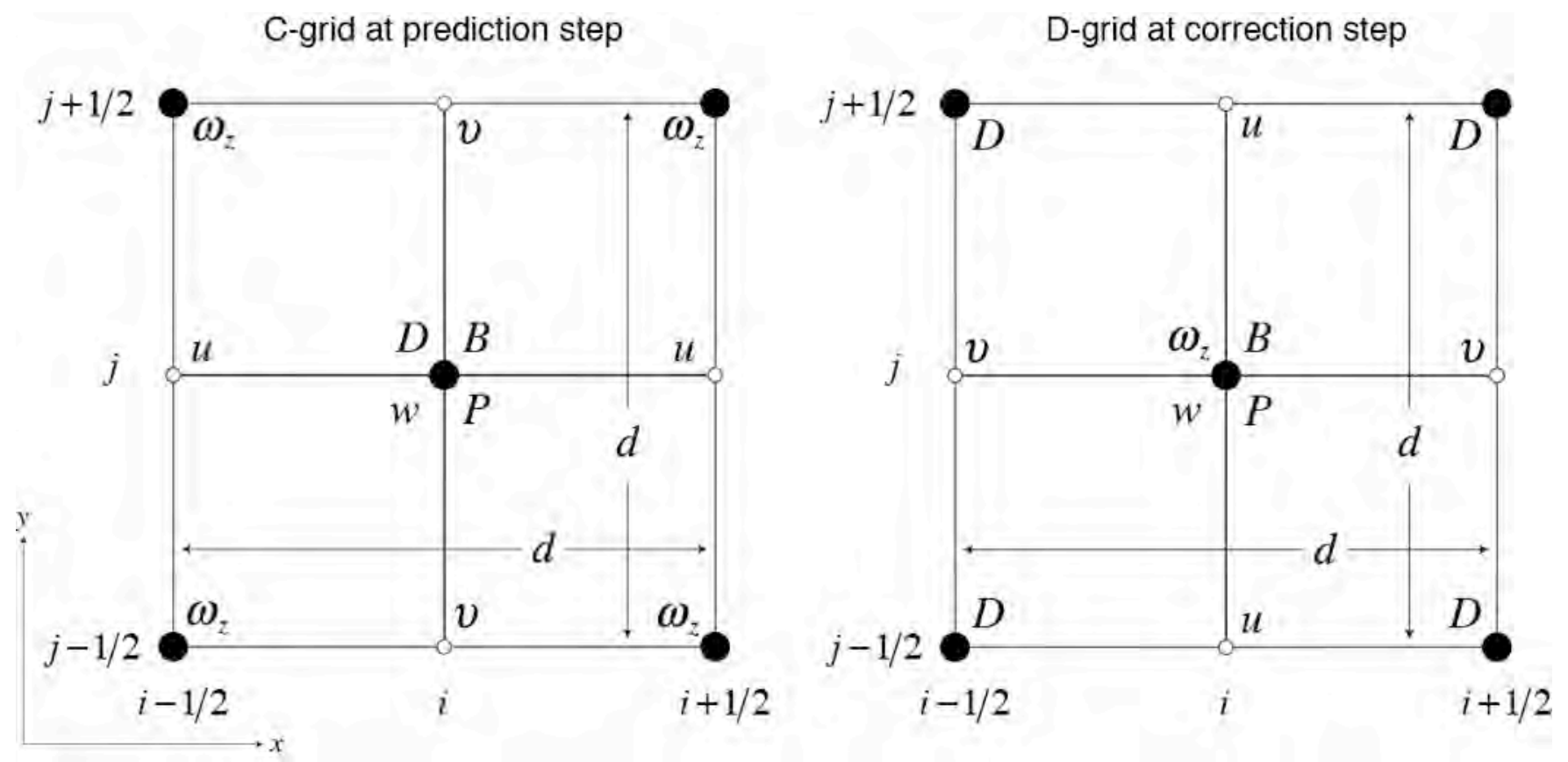

CD grid

\section{Inertia-gravity modes}

Derivation of equations: Predictor step on C grid / Corrector step on D grid:

Vorticity equation from:

$$
\frac{\partial}{\partial t}\left(\omega_{z}\right)_{i+1 / 2, j+1 / 2}=-f \frac{1}{4}\left(D_{i+1, j+1}+D_{i+1, j}+D_{i, j+1}+D_{i, j}\right)
$$

Divergence equation from (M.8):

$$
\frac{\partial}{\partial t} D_{i, j}=-\frac{P_{i+1, j}+P_{i-1, j}+P_{i, j+1}+P_{i, j-1}-4 P_{i, j}}{d^{2}}+f \frac{1}{4}\left[\left(\omega_{z}\right)_{i+1 / 2, j+1 / 2}+\left(\omega_{z}\right)_{i-1 / 2, j+1 / 2}+\left(\omega_{z}\right)_{i+1 / 2, j-1 / 2}+\left(\omega_{z}\right)_{i-1 / 2, j-1 / 2}\right]
$$


Vertical momentum equation:

$$
\frac{\partial w_{i, j}}{\partial t}=-\left[\frac{\partial}{\partial z}-\left(\frac{1}{2 \rho_{0}} \frac{\partial \rho_{0}}{\partial z}\right)\right] P_{i, j}+B_{i, j}
$$

Thermodynamic equation:

$$
\frac{\partial B_{i, j}}{\partial t}=-N^{2} w_{i, j}
$$

Mass continuity equation:

$$
D_{i, j}+\left[\frac{\partial}{\partial z}+\left(\frac{1}{2 \rho_{0}} \frac{\partial \rho_{0}}{\partial z}\right)\right] w_{i, j}=0
$$

From (4)-(6):

$$
\left(\frac{\partial^{2}}{\partial t^{2}}+N^{2}\right) D_{i, j}-\left[\frac{\partial^{2}}{\partial z^{2}}-\left(\frac{1}{2 \rho_{0}} \frac{\partial \rho_{0}}{\partial z}\right)^{2}\right] \frac{\partial}{\partial t} P_{i, j}=0
$$

Define:

$\phi_{i, j}(z, t) \equiv \operatorname{Re}\left\{\hat{\phi}(t) e^{i(k d i+\ell d j+m z)}\right\} \quad$ and $\quad \phi_{i+1 / 2, j+1 / 2}(z, t) \equiv \operatorname{Re}\left\{\hat{\phi}(t) e^{i[k d(i+1 / 2)+\ell d(j+1 / 2)+m z]}\right\}$

Using (8) in (1):

In (9)-(10): 


$$
\begin{aligned}
\mu & \equiv \frac{1}{4}\left(e^{-i k \frac{d}{2}} e^{-i \ell \frac{d}{2}}+e^{i k \frac{d}{2}} e^{-i \ell \ell \frac{d}{2}}+e^{-i k \frac{d}{2}} e^{i \ell \frac{d}{2}}+e^{i k \frac{d}{2}} e^{i \ell \frac{d}{2}}\right) \\
& =\frac{1}{4}\left[\left(e^{-i k \frac{d}{2}}+e^{i k \frac{d}{2}}\right)\left(e^{-i \ell \frac{d}{2}}+e^{i \ell \frac{d}{2}}\right)\right]=\cos \left(k \frac{d}{2}\right) \cos \left(\ell \frac{d}{2}\right)
\end{aligned}
$$

$\ln (10)$ :

$$
\begin{aligned}
\frac{e^{i k d}+e^{-i k d}+e^{i \ell d}+e^{-i \ell d}-4}{d^{2}} & =\frac{2 \cos (k d)+2 \cos (\ell d)-4}{d^{2}} \\
= & \left(\frac{-\sin ^{2}\left(\frac{1}{2} k d\right)}{\left(\frac{1}{2} k d\right)^{2}} k^{2}+\frac{-\sin ^{2}\left(\frac{1}{2} \ell d\right)}{\left(\frac{1}{2} \ell d\right)^{2}} \ell^{2}\right)
\end{aligned}
$$

Rewrite (13):

$$
\frac{e^{i k d}+e^{-i k d}+e^{i \ell d}+e^{-i \ell d}-4}{d^{2}}=-\xi^{2} k^{2}-\eta^{2} \ell^{2}
$$

where

$$
\xi \equiv \frac{\sin \left(\frac{1}{2} k d\right)}{\frac{1}{2} k d} \quad \text { and } \quad \eta \equiv \frac{\sin \left(\frac{1}{2} \ell d\right)}{\frac{1}{2} \ell d}
$$

\section{C-grid equations:}

$$
\begin{gathered}
\frac{\partial}{\partial t} \hat{\omega}_{z}=-\mu f \hat{D} \\
\frac{\partial}{\partial t} \hat{D}=\left(\xi^{2} k^{2}+\eta^{2} \ell^{2}\right) \hat{P}+\mu f \hat{\omega}_{z} \\
\frac{\partial}{\partial t} \hat{w}=-\left[\frac{\partial}{\partial z}-\left(\frac{1}{2 \rho_{0}} \frac{\partial \rho_{0}}{\partial z}\right)\right] \hat{P}+\hat{B} \\
\hat{D}+\left[\frac{\partial}{\partial z}+\left(\frac{1}{2 \rho_{0}} \frac{\partial \rho_{0}}{\partial z}\right)\right] \hat{w}=0
\end{gathered}
$$


D-grid equations:

$$
\begin{gathered}
\frac{\partial}{\partial t} \hat{\omega}_{z}=-\mu f \hat{D} \\
\frac{\partial}{\partial t} \hat{D}=\mu\left(\xi^{2} k^{2}+\eta^{2} \ell^{2}\right) \hat{P}+\mu f \hat{\omega}_{z}
\end{gathered}
$$

$\mu\left(\xi^{2} k^{2}+\eta^{2} \ell^{2}\right) \hat{P}$ is multiplied by $\mu$ because $\mathrm{P}$ is defined at the cell centers while Eq. (D.2) is applied to the cell corners, thus $P$ is averaged.

$$
\begin{gathered}
\frac{\partial}{\partial t} \hat{w}=-\left[\frac{\partial}{\partial z}-\left(\frac{1}{2 \rho_{0}} \frac{\partial \rho_{0}}{\partial z}\right)\right] \hat{P}+\hat{B} \\
\frac{\partial}{\partial t} \hat{B}=-N^{2} \hat{w} \\
\mu \hat{D}+\left[\frac{\partial}{\partial z}+\left(\frac{1}{2 \rho_{0}} \frac{\partial \rho_{0}}{\partial z}\right)\right] \hat{w}=0
\end{gathered}
$$

$\mu$ is multiplied by $\mathrm{D}$ because $\mathrm{D}$ is defined at the corners while Wq. (D.5) is applied to the cell centers, thus $D$ is averaged.

\section{Predictor step on the $C$ grid:}

C.1 is temporally discretized and modified in the CD grid as follows: Variables with (n) and $(n+1)$ are defined at the $D$ grid. Variables with $(*)$ are defined at the $C$ grid.

$$
\hat{\omega}_{z}^{(*)}=\mu \hat{\omega}_{z}^{(\mathrm{n})}-\frac{1}{2} \tau f \hat{D}^{(\mathrm{n})}
$$

$\hat{\omega}_{z}^{(*)}$ is defined at the $C$ grid (thus, corners of the CD grid), $\hat{\omega}_{z}^{(\mathrm{n})}$ is defined at the centers of the CD grid, thus it is averaged, i.e. multiplied by $\mu . \hat{D}^{(\mathrm{n})}$ is defined at the corners, thus it is not averaged. $\tau$ is the time step. At the predictor step, the time is advanced half-a-timestep. 


$$
\hat{D}^{(*)}=\mu \hat{D}^{(\mathrm{n})}+\frac{1}{2} \tau\left[\left(\xi^{2} k^{2}+\eta^{2} \ell^{2}\right) \hat{P}_{C}+f \hat{\omega}_{z}^{(\mathrm{n})}\right]
$$

$\hat{D}^{(*)}$ is defined at the centers. $\hat{D}^{(n)}$ is defined at the corners, thus it is averaged to center (multiplied by $\mu$ ). $\hat{P}_{C}$ is defined at the centers and it is a diagnostic variables, thus no time stamp. $\hat{\omega}_{z}^{(\mathrm{n})}$ is defined at the centers of the CD grid, thus it is not averaged.

$$
\begin{gathered}
\hat{w}^{(*)}=\hat{w}^{(\mathrm{n})}+\frac{1}{2} \tau\left\{-\left[\frac{\partial}{\partial z}-\left(\frac{1}{2 \rho_{0}} \frac{\partial \rho_{0}}{\partial z}\right)\right] \hat{P}_{C}+\hat{B}^{(\mathrm{n})}\right\} \\
\hat{B}^{(*)}=\hat{B}^{(\mathrm{n})}-\frac{1}{2} \tau N^{2} \hat{w}^{(\mathrm{n})} \\
\hat{D}^{(*)}+\left[\frac{\partial}{\partial z}+\left(\frac{1}{2 \rho_{0}} \frac{\partial \rho_{0}}{\partial z}\right)\right] \hat{w}^{(*)}=0
\end{gathered}
$$

\section{Corrector step on the $D$ grid:}

D. 1 is temporally discretized and modified in the CD grid as follows: Variables with (n) and $(\mathrm{n}+1)$ are defined at the $\mathrm{D}$ grid. Variables with $(*)$ are defined at the $\mathrm{C}$ grid.

$$
\hat{\omega}_{z}^{(\mathrm{n}+1)}=\hat{\omega}_{z}^{(\mathrm{n})}-\tau f \hat{D}^{(*)}
$$

$\hat{D}^{(*)}$ is defined at the corners, thus no averaging is needed in $(C D .1 n+1)$.

D.2 is temporally discretized and modified in the CD grid as follows:

$$
\hat{D}^{(\mathrm{n}+1)}=\hat{D}^{(\mathrm{n})}+\tau\left[\mu\left(\xi^{2} k^{2}+\eta^{2} \ell^{2}\right) \hat{P}_{D}+f \hat{\omega}_{z}^{(*)}\right]
$$

$\hat{P}_{D}$ needs to be averaged to the corners, thus multiplied by $\mu$. No averaging is needed for $\hat{\omega}_{z}^{(*)}$ in $(C D .2 n+1)$.

$$
\hat{w}^{(\mathrm{n}+1)}=\hat{w}^{(\mathrm{n})}+\tau\left\{-\left[\frac{\partial}{\partial z}-\left(\frac{1}{2 \rho_{0}} \frac{\partial \rho_{0}}{\partial z}\right)\right] \hat{P}_{D}+\hat{B}^{(*)}\right\}
$$




$$
\begin{gathered}
\hat{B}^{(\mathrm{n}+1)}=\hat{B}^{(\mathrm{n})}-\tau N^{2} \hat{w}^{(*)} \\
\mu \hat{D}^{(\mathrm{n})}+\left[\frac{\partial}{\partial z}+\left(\frac{1}{2 \rho_{0}} \frac{\partial \rho_{0}}{\partial z}\right)\right] \hat{w}^{(\mathrm{n})}=0 \text { and } \mu \hat{D}^{(\mathrm{n}+1)}+\left[\frac{\partial}{\partial z}+\left(\frac{1}{2 \rho_{0}} \frac{\partial \rho_{0}}{\partial z}\right)\right] \hat{w}^{(\mathrm{n}+1)}=0 \\
(\mathrm{CD} .5 \mathrm{n}+1)
\end{gathered}
$$

$\hat{D}^{(\mathrm{n})}$ is multiplied by $\mu$ because $\hat{D}^{(\mathrm{n})}$ needs to be averaged to the centers. 


\section{Scheme I:}

Using $\frac{\partial}{\partial z}+\left(\frac{1}{2 \rho_{0}} \frac{\partial \rho_{0}}{\partial z}\right)=\left(\underset{\sim}{i m}-\frac{1}{2 H}\right)$ and $\frac{\partial}{\partial z}-\left(\frac{1}{2 \rho_{0}} \frac{\partial \rho_{0}}{\partial z}\right)=\left(\underset{\sim}{i} m+\frac{1}{2 H}\right)$ in the equations:

Predictor step on the C grid:

$$
\begin{aligned}
& \hat{\omega}_{z}^{(*)}=\mu \hat{\omega}_{z}^{(\mathrm{n})}-\frac{1}{2} \tau f \hat{D}^{(\mathrm{n})} \\
& \hat{D}^{(*)}=\mu \hat{D}^{(\mathrm{n})}+\frac{1}{2} \tau\left[\left(\xi^{2} k^{2}+\eta^{2} \ell^{2}\right) \hat{P}_{C}+f \hat{\omega}_{z}^{(\mathrm{n})}\right] \\
& \hat{w}^{(*)}=\hat{w}^{(\mathrm{n})}+\frac{1}{2} \tau\left[-\left(\underset{\sim}{i m}+\frac{1}{2 H}\right) \hat{P}_{C}+\hat{B}^{(\mathrm{n})}\right] \\
& \hat{B}^{(*)}=\hat{B}^{(\mathrm{n})}-\frac{1}{2} \tau N^{2} \hat{w}^{(\mathrm{n})} \\
& \hat{D}^{(*)}+\left(\underset{\sim}{i} m-\frac{1}{2 H}\right) \hat{w}^{(*)}=0
\end{aligned}
$$

\section{Corrector step on the D grid:}

$$
\begin{aligned}
& \hat{\omega}_{z}^{(\mathrm{n}+1)}=\hat{\omega}_{z}^{(\mathrm{n})}-\tau f \hat{D}^{(*)} \\
& \hat{D}^{(\mathrm{n}+1)}=\hat{D}^{(\mathrm{n})}+\tau\left[\mu\left(\xi^{2} k^{2}+\eta^{2} \ell^{2}\right) \hat{P}_{D}+f \hat{\omega}_{z}^{(*)}\right] \\
& \hat{w}^{(\mathrm{n}+1)}=\hat{w}^{(\mathrm{n})}+\tau\left\{-\left(\underset{\sim}{i} m+\frac{1}{2 H}\right) \hat{P}_{D}+\hat{B}^{(*)}\right\} \\
& \hat{B}^{(n+1)}=\hat{B}^{(\mathrm{n})}-\tau N^{2} \hat{w}^{(*)} \\
& \mu \hat{D}^{(\mathrm{n})}+\left(\underset{\sim}{\operatorname{im}}-\frac{1}{2 H}\right) \hat{w}^{(\mathrm{n})}=0 \text { and } \mu \hat{D}^{(\mathrm{n}+1)}+\left(\underset{\sim}{\operatorname{i} m}-\frac{1}{2 H}\right) \hat{w}^{(\mathrm{n}+1)}=0
\end{aligned}
$$

Assume $\hat{P}=\hat{P}_{C}=\hat{P}_{D}$ in scheme I.

Using (2) in (6), and:

$$
\begin{gathered}
L^{2} \equiv \xi^{2} k^{2}+\eta^{2} \ell^{2} \\
\hat{\omega}_{z}^{(\mathrm{n}+1)}=\left(1-\frac{1}{2} \tau^{2} f^{2}\right) \hat{\omega}_{z}^{(\mathrm{n})}-\mu \tau f \hat{D}^{(\mathrm{n})}-\frac{1}{2} \tau^{2} f L^{2} \hat{P}
\end{gathered}
$$


Using (1) in (7):

$$
\begin{gathered}
\hat{D}^{(\mathrm{n}+1)}=\left(1-\frac{1}{2} \tau^{2} f^{2}\right) \hat{D}^{(\mathrm{n})}+\mu \tau f \hat{\boldsymbol{\omega}}_{z}^{(\mathrm{n})}+\mu \tau L^{2} \hat{P} \\
\hat{w}^{(\mathrm{n}+1)}=\left(1-\frac{1}{2} \tau^{2} N^{2}\right) \hat{w}^{(n)}+\tau \hat{B}^{(n)}-\tau\left(\underset{\sim}{\underset{i}{i}} m+\frac{1}{2 H}\right) \hat{P} \\
\hat{B}^{(\mathrm{n}+1)}=\left(1-\frac{1}{2} \tau^{2} N^{2}\right) \hat{B}^{(\mathrm{n})}-\tau N^{2} \hat{w}^{(\mathrm{n})}+\frac{1}{2} \tau^{2} N^{2}\left(\underset{\sim}{i} m+\frac{1}{2 H}\right) \hat{P} \\
\mu \hat{D}^{(\mathrm{n})}+\left(\underset{\sim}{i} m-\frac{1}{2 H}\right) \hat{w}^{(\mathrm{n})}=0 \quad \text { and } \mu \hat{D}^{(\mathrm{n}+1)}+\left(\underset{\sim}{i} m-\frac{1}{2 H}\right) \hat{w}^{(\mathrm{n}+1)}=0
\end{gathered}
$$

Repeat (13 and (14):

$$
\begin{gathered}
\hat{D}^{(\mathrm{n}+1)}=\left(1-\frac{1}{2} \tau^{2} f^{2}\right) \hat{D}^{(\mathrm{n})}+\mu \tau f \hat{\omega}_{z}^{(\mathrm{n})}+\mu \tau L^{2} \hat{P} \\
\hat{w}^{(\mathrm{n}+1)}=\left(1-\frac{1}{2} \tau^{2} N^{2}\right) \hat{w}^{(n)}+\tau \hat{B}^{(n)}-\tau\left(\underset{\sim}{i m}+\frac{1}{2 H}\right) \hat{P}
\end{gathered}
$$

Using (16) in (14):

$$
-\mu \hat{D}^{(\mathrm{n}+1)}=-\left(1-\frac{1}{2} \tau^{2} N^{2}\right) \mu \hat{D}^{(\mathrm{n})}+\tau\left(\underset{\sim}{i m}-\frac{1}{2 H}\right) \hat{B}^{(\mathrm{n})}+\tau\left(m^{2}+\frac{1}{4 H^{2}}\right) \hat{P}
$$

Using (16) in (15):

$$
\left(\underset{\sim}{i m}-\frac{1}{2 H}\right) \hat{B}^{(\mathrm{n}+1)}=\left(1-\frac{1}{2} \tau^{2} N^{2}\right)\left(\underset{\sim}{i m}-\frac{1}{2 H}\right) \hat{B}^{(\mathrm{n})}+\mu \tau N^{2} \hat{D}^{(\mathrm{n})}-\frac{1}{2} \tau^{2} N^{2}\left(m^{2}+\frac{1}{4 H^{2}}\right) \hat{P}
$$

\section{Balanced state solution:}

$\mathrm{D}=0, \partial / \partial t=0$ in (13), (19) and (20):

$$
\begin{gathered}
0=f \hat{\omega}_{z}^{(\mathrm{n})}+L^{2} \hat{P} \\
0=\left(\underset{\sim}{i} m-\frac{1}{2 H}\right) \hat{B}^{(\mathrm{n})}+\left(m^{2}+\frac{1}{4 H^{2}}\right) \hat{P}
\end{gathered}
$$




$$
0=\left(\underset{\sim}{i} m-\frac{1}{2 H}\right) \hat{B}^{(\mathrm{n})}+\left(m^{2}+\frac{1}{4 H^{2}}\right) \hat{P}
$$

Using (22) in (21):

$$
0=f\left(\underset{\sim}{i} m+\frac{1}{2 H}\right) \hat{\omega}_{z}^{(\mathrm{n})}+L^{2} \hat{B}^{(\mathrm{n})}
$$

Geostrophic balance: $0=f \hat{\omega}_{z}^{(\mathrm{n})}+L^{2} \hat{P}$ and $0=f\left(\underset{\sim}{i m}+\frac{1}{2 H}\right) \hat{\omega}_{z}^{(\mathrm{n})}+L^{2} \hat{B}^{(\mathrm{n})}$

Quasi-static approximation: $0=\hat{B}^{(\mathrm{n})}-\left(\underset{\sim}{i} m+\frac{1}{2 H}\right) \hat{P}$

Eqs. (12), (13), (19) and (20) form the system of equations (Scheme I):

$$
\begin{aligned}
& \hat{\omega}_{z}^{(\mathrm{n}+1)}=\left(1-\frac{1}{2} \tau^{2} f^{2}\right) \hat{\omega}_{z}^{(\mathrm{n})}-\mu \tau f \hat{D}^{(\mathrm{n})}-\frac{1}{2} \tau^{2} f L^{2} \hat{P} \\
& \hat{D}^{(\mathrm{n}+1)}=\left(1-\frac{1}{2} \tau^{2} f^{2}\right) \hat{D}^{(\mathrm{n})}+\mu \tau f \hat{\omega}_{z}^{(\mathrm{n})}+\mu \tau L^{2} \hat{P} \\
& -\mu \hat{D}^{(\mathrm{n}+1)}=-\left(1-\frac{1}{2} \tau^{2} N^{2}\right) \mu \hat{D}^{(\mathrm{n})}+\tau\left(\underset{\sim}{i} m-\frac{1}{2 H}\right) \hat{B}^{(\mathrm{n})}+\tau\left(m^{2}+\frac{1}{4 H^{2}}\right) \hat{P} \\
& \left(\underset{\sim}{i} m-\frac{1}{2 H}\right) \hat{B}^{(\mathrm{n}+1)}=\left(1-\frac{1}{2} \tau^{2} N^{2}\right)\left(\underset{\sim}{i} m-\frac{1}{2 H}\right) \hat{B}^{(\mathrm{n})}+\mu \tau N^{2} \hat{D}^{(\mathrm{n})}-\frac{1}{2} \tau^{2} N^{2}\left(m^{2}+\frac{1}{4 H^{2}}\right) \hat{P}
\end{aligned}
$$

Definitions:

$$
\begin{gathered}
\hat{\Phi}^{(\mathrm{n}+1)}=e^{-i v \tau} \hat{\boldsymbol{\Phi}}^{(\mathrm{n})} \\
\sigma_{f} \equiv 1-\frac{1}{2} \tau^{2} f^{2}, \sigma_{N} \equiv 1-\frac{1}{2} \tau^{2} N^{2} \text { and } \sigma_{m}^{2} \equiv m^{2}+\frac{1}{4 H^{2}}
\end{gathered}
$$

Using (31) and (32) in (27), (28), (29) and (30):

$$
\begin{gathered}
\left(e^{-i v \tau}-\sigma_{f}\right) \hat{\omega}_{z}=-\mu \tau f \hat{D}-\frac{1}{2} \tau^{2} f L^{2} \hat{P} \\
\left(e^{-i v \tau}-\sigma_{f}\right) \hat{D}=\mu \tau f \hat{\omega}_{z}+\mu \tau L^{2} \hat{P} \\
\mu\left(e^{-i v \tau}-\sigma_{N}\right) \hat{D}=-\tau\left({\underset{\sim}{i}}_{\sim}-\frac{1}{2 H}\right) \hat{B}-\tau \sigma_{m}^{2} \hat{P}
\end{gathered}
$$




$$
\left(e^{-i v \tau}-\sigma_{N}\right)\left(\underset{\sim}{i m}-\frac{1}{2 H}\right) \hat{B}=\mu \tau N^{2} \hat{D}-\frac{1}{2} \tau^{2} N^{2} \sigma_{m}^{2} \hat{P}
$$

Eliminate $\hat{\omega}_{z}$ between (33) and (34):

$$
\left[\left(e^{-i v \tau}-\sigma_{f}\right)^{2}+\mu^{2} \tau^{2} f^{2}\right] \hat{D}=\mu \tau\left(e^{-i v \tau}-1\right) L^{2} \hat{P}
$$

Eliminate $\hat{B}$ between (35) and (36):

$$
-\mu\left[\left(e^{-i v \tau}-\sigma_{N}\right)^{2}+\tau^{2} N^{2}\right] \hat{D}=\tau\left(e^{-i v \tau}-1\right) \sigma_{m}^{2} \hat{P}
$$

Eliminate $\hat{D}$ between (37) and (38):

$$
0=\mu^{2}\left(e^{-i v \tau}-1\right)\left[\left(e^{-i v \tau}-\sigma_{N}\right)^{2}+\tau^{2} N^{2}\right] L^{2} \hat{P}+\left(e^{-i v \tau}-1\right)\left[\left(e^{-i v \tau}-\sigma_{f}\right)^{2}+\mu^{2} \tau^{2} f^{2}\right] \sigma_{m}^{2} \hat{P}
$$

Nontrivial solutions:

$$
0=\mu^{2}\left(e^{-i v \tau}-1\right)\left[\left(e^{-i v \tau}-\sigma_{N}\right)^{2}+\tau^{2} N^{2}\right] L^{2}+\left(e^{-i v \tau}-1\right)\left[\left(e^{-i v \tau}-\sigma_{f}\right)^{2}+\mu^{2} \tau^{2} f^{2}\right] \sigma_{m}^{2}
$$

Solution 1:

$$
e^{-i v \tau}-1=0
$$

Solution 2:

$$
0=\mu^{2}\left[\left(e^{-i v \tau}-\sigma_{N}\right)^{2}+\tau^{2} N^{2}\right] L^{2}+\left[\left(e^{-i v \tau}-\sigma_{f}\right)^{2}+\mu^{2} \tau^{2} f^{2}\right] \sigma_{m}^{2}
$$

Definition:

$$
v \equiv v_{r}+\underset{\sim}{i} v_{i}
$$

Using (42) in (41a) and (41b):

Solution 1:

$$
e^{v_{i} \tau}\left[\cos \left(v_{r} \tau\right)-\underset{\sim}{i} \sin \left(v_{r} \tau\right)\right]-1=0
$$




$$
e^{v_{i} \tau} \cos \left(v_{r} \tau\right)-1=0
$$

and

$$
e^{v_{i} \tau} \sin \left(v_{r} \tau\right)=0
$$

\section{Solution 2:}

$e^{2 v_{i} \tau}\left(\mu^{2} L^{2}+\sigma_{m}^{2}\right) \cos \left(2 v_{r} \tau\right)-2 e^{v_{i} \tau}\left(\mu^{2} \sigma_{N} L^{2}+\sigma_{f} \sigma_{m}^{2}\right) \cos \left(v_{r} \tau\right)+\left(\sigma_{N}^{2}+\tau^{2} N^{2}\right) \mu^{2} L^{2}+\left(\sigma_{f}^{2}+\tau^{2} \mu^{2} f^{2}\right) \sigma_{m}^{2}=0$

and

$$
e^{v_{i} \tau}=\frac{2\left(\mu^{2} \sigma_{N} L^{2}+\sigma_{f} \sigma_{m}^{2}\right)}{\left(\mu^{2} L^{2}+\sigma_{m}^{2}\right)} \frac{\sin \left(v_{r} \tau\right)}{\sin \left(2 v_{r} \tau\right)}
$$

\section{Newton Raphson:}

$$
\begin{aligned}
& F\left(v_{r}\right)=e^{2 v_{i} \tau}\left(\mu^{2} L^{2}+\sigma_{m}^{2}\right) \cos \left(2 v_{r} \tau\right)-2 e^{v_{i} \tau}\left(\mu^{2} \sigma_{N} L^{2}+\sigma_{f} \sigma_{m}^{2}\right) \cos \left(v_{r} \tau\right) \\
& +\left(\sigma_{N}^{2}+\tau^{2} N^{2}\right) \mu^{2} L^{2}+\left(\sigma_{f}^{2}+\tau^{2} \mu^{2} f^{2}\right) \sigma_{m}^{2} \\
& \frac{\partial F}{\partial v_{r}}=\frac{\partial}{\partial v_{r}}\left(e^{2 v_{i} \tau}\right)\left(\mu^{2} L^{2}+\sigma_{m}^{2}\right) \cos \left(2 v_{r} \tau\right)-e^{2 v_{i} \tau}\left(\mu^{2} L^{2}+\sigma_{m}^{2}\right) 2 \tau \sin \left(2 v_{r} \tau\right) \\
& -2 \frac{\partial}{\partial v_{r}}\left(e^{v_{i} \tau}\right)\left(\mu^{2} \sigma_{N} L^{2}+\sigma_{f} \sigma_{m}^{2}\right) \cos \left(v_{r} \tau\right)+2 e^{v_{i} \tau}\left(\mu^{2} \sigma_{N} L^{2}+\sigma_{f} \sigma_{m}^{2}\right) \tau \sin \left(v_{r} \tau\right) \\
& \frac{\partial}{\partial v_{r}}\left(e^{v_{i} \tau}\right)=\frac{2\left(\mu^{2} \sigma_{N} L^{2}+\sigma_{f} \sigma_{m}^{2}\right)}{\left(\mu^{2} L^{2}+\sigma_{m}^{2}\right)} \frac{\tau \cos \left(v_{r} \tau\right) \sin \left(2 v_{r} \tau\right)-2 \tau \cos \left(2 v_{r} \tau\right) \sin \left(v_{r} \tau\right)}{\sin ^{2}\left(2 v_{r} \tau\right)} \\
& \left(v_{r}\right)_{1}=\left(v_{r}\right)_{0}-\frac{F\left[\left(v_{r}\right)_{0}\right]}{\left(\frac{\partial F}{\partial v_{r}}\right)_{\left(v_{r}\right)_{0}}}
\end{aligned}
$$

\section{Balance solution:}


Using $\hat{\omega}_{z}^{(\mathrm{n}+1)}-\hat{\omega}_{z}^{(\mathrm{n})}=0, \hat{D}=0$ (therefore, $\mathrm{w}=0$ ) and $\hat{B}^{(\mathrm{n}+1)}-\hat{B}^{(\mathrm{n})}=0$ in $(27)-(30)$ :

$0=-f \hat{\omega}_{z}^{(\mathrm{n})}-L^{2} \hat{P}$

$0=f \hat{\omega}_{z}^{(\mathrm{n})}+L^{2} \hat{P}$

$0=\left(\underset{\sim}{i m}-\frac{1}{2 H}\right) \hat{B}^{(\mathrm{n})}+\left(m^{2}+\frac{1}{4 H^{2}}\right) \hat{P} \quad$ or $\quad 0=\hat{B}^{(\mathrm{n})}-\left(\underset{\sim}{i} m+\frac{1}{2 H}\right) \hat{P}$

$0=-\left(\underset{\sim}{i} m-\frac{1}{2 H}\right) \hat{B}^{(\mathrm{n})}-\left(m^{2}+\frac{1}{4 H^{2}}\right) \hat{P} \quad$ or $\quad 0=\hat{B}^{(\mathrm{n})}-\left(\underset{\sim}{i} m+\frac{1}{2 H}\right) \hat{P}$

Using (48) and (49) in (46) and (47):

$$
0=L^{2} \hat{B}^{(\mathrm{n})}+f\left(\underset{\sim}{i m}+\frac{1}{2 H}\right) \hat{\boldsymbol{\omega}}_{z}^{(\mathrm{n})}
$$

There is a unique balance with scheme I. 


\section{Scheme II:}

Prediction-step on C grid:

$$
\begin{aligned}
& \hat{\omega}_{z}^{(*)}=\mu \hat{\omega}_{z}^{(\mathrm{n})}-\frac{\tau}{2} f \hat{D}^{(\mathrm{n})} \\
& \hat{D}^{(*)}=\mu \hat{D}^{(\mathrm{n})}+\frac{\tau}{2}\left(f \hat{\omega}_{z}^{(\mathrm{n})}+L^{2} \hat{P}_{C}\right) \\
& \hat{w}^{(*)}=\hat{w}^{(\mathrm{n})}+\frac{\tau}{2}\left[-\left(\underset{\sim}{\underset{\sim}{m}} m+\frac{1}{2 H}\right) \hat{P}_{C}+\hat{B}^{(\mathrm{n})}\right] \\
& \hat{B}^{(*)}=\hat{B}^{(\mathrm{n})}-\frac{\tau}{2} N^{2} \hat{w}^{(\mathrm{n})} \\
& \hat{D}^{(*)}+\left(\underset{\sim}{\underset{\sim}{i}} m-\frac{1}{2 H}\right) \hat{w}^{(*)}=0
\end{aligned}
$$

\section{Corrector-step on D grid:}

$$
\begin{aligned}
& \hat{\omega}_{z}^{(\mathrm{n}+1)}=\hat{\omega}_{z}^{(\mathrm{n})}-\tau f \hat{D}^{(*)} \\
& \hat{D}^{(\mathrm{n}+1)}=\hat{D}^{(\mathrm{n})}+\tau\left(f \hat{\omega}_{z}^{(*)}+\mu L^{2} \hat{P}_{D}\right) \\
& \hat{w}^{(\mathrm{n}+1)}=\hat{w}^{(\mathrm{n})}+\tau\left[-\left(\underset{\sim}{\operatorname{in}}+\frac{1}{2 H}\right) \hat{P}_{D}+\hat{B}^{(*)}\right] \\
& \hat{B}^{(\mathrm{n}+1)}=\hat{B}^{(\mathrm{n})}-\tau N^{2} \hat{w}^{(*)} \\
& \mu \hat{D}^{(\mathrm{n}+1)}+\left(\underset{\sim}{i m}-\frac{1}{2 H}\right) \hat{w}^{(\mathrm{n}+1)}=0
\end{aligned}
$$

\section{Prediction-step equations:}

Eliminating $\hat{P}_{C}$ between (61) and (62)

Eq. (61) becomes

$$
\begin{aligned}
-\left(L^{2}+m^{2}+\frac{1}{4 H^{2}}\right) \hat{D}^{(*)} & =-\mu\left(m^{2}+\frac{1}{4 H^{2}}\right) \hat{D}^{(\mathrm{n})} \\
& -\frac{\tau}{2} f\left(m^{2}+\frac{1}{4 H^{2}}\right) \hat{\omega}_{z}^{(\mathrm{n})}+L^{2}\left(\underset{\sim}{\operatorname{im}}-\frac{1}{2 H}\right) \hat{w}^{(\mathrm{n})}+\frac{\tau}{2}\left(\underset{\sim}{\underset{\sim}{m}}-\frac{1}{2 H}\right) L^{2} \hat{B}^{(\mathrm{n})}
\end{aligned}
$$

Eq. (62) becomes

$$
\begin{aligned}
\left(L^{2}+m^{2}+\frac{1}{4 H^{2}}\right) & \left(\underset{\sim}{i m}-\frac{1}{2 H}\right) \hat{w}^{(*)}=-\mu\left(m^{2}+\frac{1}{4 H^{2}}\right) \hat{D}^{(\mathrm{n})} \\
& -\frac{\tau}{2} f\left(m^{2}+\frac{1}{4 H^{2}}\right) \hat{\omega}_{z}^{(\mathrm{n})}+L^{2}\left(\underset{\sim}{\underset{i}{m}}-\frac{1}{2 H}\right) \hat{w}^{(\mathrm{n})}+\frac{\tau}{2}\left(\underset{\sim}{\underset{\sim}{m}}-\frac{1}{2 H}\right) L^{2} \hat{B}^{(\mathrm{n})}
\end{aligned}
$$

\section{Corrector-step equations:}

Using (71) in (65): 


$$
\begin{aligned}
\left(L^{2}+m^{2}+\frac{1}{4 H^{2}}\right) \hat{\omega}_{z}^{(\mathrm{n}+1)}= & {\left[\left(L^{2}+m^{2}+\frac{1}{4 H^{2}}\right)-\frac{\tau^{2} f^{2}}{2}\left(m^{2}+\frac{1}{4 H^{2}}\right)\right] \hat{\omega}_{z}^{(\mathrm{n})} } \\
& -\tau f \mu\left(L^{2}+m^{2}+\frac{1}{4 H^{2}}\right) \hat{D}^{(\mathrm{n})}+\tau f \frac{\tau}{2}\left(\underset{\sim}{i m}-\frac{1}{2 H}\right) L^{2} \hat{B}^{(\mathrm{n})}
\end{aligned}
$$

Using (60) and (63) in (66) and eliminating $\hat{P}_{D}$ and $w^{(\mathrm{n}+1)}\left(\right.$ and $\left.w^{(\mathrm{n})}\right)$ between (66), (67) and (69) $\left[\mu \hat{D}^{(\mathrm{n})}+\left(\underset{\sim}{i} m-\frac{1}{2 H}\right) \hat{w}^{(\mathrm{n})}=0\right]:$

$$
\begin{array}{r}
\left(\mu^{2} L^{2}+m^{2}+\frac{1}{4 H^{2}}\right) \hat{D}^{(\mathrm{n}+1)}=\left[\left(1-\frac{\tau^{2} f^{2}}{2}\right)\left(m^{2}+\frac{1}{4 H^{2}}\right)+\mu^{2}\left(1-\frac{\tau^{2} N^{2}}{2}\right) L^{2}\right] \hat{D}^{(\mathrm{n})} \\
+\tau f \mu\left(m^{2}+\frac{1}{4 H^{2}}\right) \hat{\omega}_{z}^{(\mathrm{n})}-\mu \tau\left(\underset{\sim}{i m}-\frac{1}{2 H}\right) L^{2} \hat{B}^{(\mathrm{n})}
\end{array}
$$

Using (62) and (69) in (68):

$$
\begin{array}{r}
\left(L^{2}+m^{2}+\frac{1}{4 H^{2}}\right)\left(\underset{\sim}{i} m-\frac{1}{2 H}\right) \hat{B}^{(\mathrm{n}+1)}=\left[\left(L^{2}+m^{2}+\frac{1}{4 H^{2}}\right)-\frac{\tau^{2} N^{2}}{2} L^{2}\right]\left(\underset{\sim}{i} m-\frac{1}{2 H}\right) \hat{B}^{(\mathrm{n})} \\
+\mu \tau N^{2}\left(L^{2}+m^{2}+\frac{1}{4 H^{2}}\right) \hat{D}^{(\mathrm{n})}+\frac{\tau}{2} f \tau N^{2}\left(m^{2}+\frac{1}{4 H^{2}}\right) \hat{\omega}_{z}^{(\mathrm{n})}
\end{array}
$$

Eqs. (73)-(75) are the equations of the system. Rewriting (73)-(75):

$$
\begin{aligned}
& \left(L^{2}+\sigma_{m}^{2}\right) \hat{\omega}_{z}^{(\mathrm{n}+1)}=\left(L^{2}+\sigma_{f} \sigma_{m}^{2}\right) \hat{\omega}_{z}^{(\mathrm{n})}-\tau f \mu\left(L^{2}+\sigma_{m}^{2}\right) \hat{D}^{(\mathrm{n})}+f \frac{\tau^{2}}{2} L^{2}\left(\underset{\sim}{i} m-\frac{1}{2 H}\right) \hat{B}^{(\mathrm{n})} \\
& \left(\mu^{2} L^{2}+\sigma_{m}^{2}\right) \hat{D}^{(\mathrm{n}+1)}=\left(\mu^{2} \sigma_{N} L^{2}+\sigma_{f} \sigma_{m}^{2}\right) \hat{D}^{(\mathrm{n})}+\tau \mu f \sigma_{m}^{2} \hat{\omega}_{z}^{(\mathrm{n})}-\tau \mu L^{2}\left(\underset{\sim}{i} m-\frac{1}{2 H}\right) \hat{B}^{(\mathrm{n})} \\
& \left(L^{2}+\sigma_{m}^{2}\right)\left(\underset{\sim}{i} m-\frac{1}{2 H}\right) \hat{B}^{(\mathrm{n}+1)}=\left(\sigma_{N} L^{2}+\sigma_{m}^{2}\right)\left(\underset{\sim}{i} m-\frac{1}{2 H}\right) \hat{B}^{(\mathrm{n})}+\mu \tau N^{2}\left(L^{2}+\sigma_{m}^{2}\right) \hat{D}^{(\mathrm{n})}+\frac{\tau}{2} f \tau N^{2} \sigma_{m}^{2} \hat{\omega}_{z}^{(\mathrm{n})}
\end{aligned}
$$

where

$$
\sigma_{f} \equiv 1-\frac{1}{2} \tau^{2} f^{2}, \sigma_{N} \equiv 1-\frac{1}{2} \tau^{2} N^{2} \text { and } \sigma_{m}^{2} \equiv m^{2}+\frac{1}{4 H^{2}}
$$

Using $\hat{\Phi}^{(\mathrm{n}+1)}=e^{-i v \tau} \hat{\Phi}^{(\mathrm{n})}$ in (77)-(79): 


$$
\begin{aligned}
& \left(L^{2}+\sigma_{m}^{2}\right) e^{-i v \tau} \hat{\omega}_{z}=\left(L^{2}+\sigma_{f} \sigma_{m}^{2}\right) \hat{\omega}_{z}-\tau f \mu\left(L^{2}+\sigma_{m}^{2}\right) \hat{D}+f \frac{\tau^{2}}{2} L^{2}\left(\underset{\sim}{i m}-\frac{1}{2 H}\right) \hat{B} \\
& \left(\mu^{2} L^{2}+\sigma_{m}^{2}\right) e^{-i v \tau} \hat{D}=\left(\mu^{2} \sigma_{N} L^{2}+\sigma_{f} \sigma_{m}^{2}\right) \hat{D}+\tau \mu f \sigma_{m}^{2} \hat{\omega}_{z}-\tau \mu L^{2}\left(\underset{\sim}{i} m-\frac{1}{2 H}\right) \hat{B} \\
& \left(L^{2}+\sigma_{m}^{2}\right)\left({\underset{\sim}{\sim}}_{i} m-\frac{1}{2 H}\right) e^{-i v \tau} \hat{B}=\left(\sigma_{N} L^{2}+\sigma_{m}^{2}\right)\left(\underset{\sim}{i} m-\frac{1}{2 H}\right) \hat{B}+\mu \tau N^{2}\left(L^{2}+\sigma_{m}^{2}\right) \hat{D}+\frac{\tau}{2} f \tau N^{2} \sigma_{m}^{2} \hat{\omega}_{z}
\end{aligned}
$$

Eliminating B between (80) and (81):

$$
\mu\left(L^{2}+\sigma_{m}^{2}\right)\left(e^{-i v \tau}-1\right) \hat{\omega}_{z}=\tau f\left[-\frac{1}{2}\left(\mu^{2} L^{2}+\sigma_{m}^{2}\right) e^{-i v \tau}-\mu^{2}\left(L^{2}+\sigma_{m}^{2}\right)+\frac{1}{2}\left(\mu^{2} \sigma_{N} L^{2}+\sigma_{f} \sigma_{m}^{2}\right)\right] \hat{D}
$$

Eliminating B between (81) and (82):

$$
\begin{array}{r}
\left\{\left[\left(L^{2}+\sigma_{m}^{2}\right) e^{-i v \tau}-\left(\sigma_{N} L^{2}+\sigma_{m}^{2}\right)\right]\left[\left(\mu^{2} L^{2}+\sigma_{m}^{2}\right) e^{-i v \tau}-\left(\mu^{2} \sigma_{N} L^{2}+\sigma_{f} \sigma_{m}^{2}\right)\right]+\mu^{2} \tau^{2} N^{2} L^{2}\left(L^{2}+\sigma_{m}^{2}\right)\right\} \hat{D}= \\
\left(L^{2}+\sigma_{m}^{2}\right)\left(e^{-i v \tau}-1\right) \tau \mu f \sigma_{m}^{2} \hat{\omega}_{z}
\end{array}
$$

Eliminating $\hat{D}$ between in (83) and (84):

$$
\begin{aligned}
& \left(L^{2}+\sigma_{m}^{2}\right)\left(e^{-i v \tau}-1\right) \mathcal{B} \hat{\omega}_{z}=\left(L^{2}+\sigma_{m}^{2}\right)\left(e^{-i v \tau}-1\right) \mathcal{A} \tau f \sigma_{m}^{2} \hat{\omega}_{z} \\
& \mathcal{A}=\tau f\left[-\frac{1}{2}\left(\mu^{2} L^{2}+\sigma_{m}^{2}\right) e^{-i v \tau}-\mu^{2}\left(L^{2}+\sigma_{m}^{2}\right)+\frac{1}{2}\left(\mu^{2} \sigma_{N} L^{2}+\sigma_{f} \sigma_{m}^{2}\right)\right] \\
& \mathcal{B}=\left[\left(L^{2}+\sigma_{m}^{2}\right) e^{-i v \tau}-\left(\sigma_{N} L^{2}+\sigma_{m}^{2}\right)\right]\left[\left(\mu^{2} L^{2}+\sigma_{m}^{2}\right) e^{-i v \tau}-\left(\mu^{2} \sigma_{N} L^{2}+\sigma_{f} \sigma_{m}^{2}\right)\right]+\mu^{2} \tau^{2} N^{2} L^{2}\left(L^{2}+\sigma_{m}^{2}\right)
\end{aligned}
$$

From (85). Nontrivial solutions:

\section{Solution 1:}

$$
e^{-i v \tau}-1=0
$$

\section{Solution 2:}

$$
\begin{array}{r}
{\left[\left(L^{2}+\sigma_{m}^{2}\right) e^{-i v \tau}-\left(\sigma_{N} L^{2}+\sigma_{m}^{2}\right)\right]\left[\left(\mu^{2} L^{2}+\sigma_{m}^{2}\right) e^{-i v \tau}-\left(\mu^{2} \sigma_{N} L^{2}+\sigma_{f} \sigma_{m}^{2}\right)\right]+\mu^{2} \tau^{2} N^{2} L^{2}\left(L^{2}+\sigma_{m}^{2}\right)=} \\
-\frac{1}{2} \tau^{2} f^{2} \sigma_{m}^{2}\left(\mu^{2} L^{2}+\sigma_{m}^{2}\right) e^{-i v \tau}-\tau^{2} f^{2} \sigma_{m}^{2} \mu^{2}\left(L^{2}+\sigma_{m}^{2}\right)+\frac{1}{2} \tau^{2} f^{2} \sigma_{m}^{2}\left(\mu^{2} \sigma_{N} L^{2}+\sigma_{f} \sigma_{m}^{2}\right)
\end{array}
$$


Arranging the terms (86b):

$$
\begin{array}{r}
{\left[\left(L^{2}+\sigma_{m}^{2}\right) e^{-i v \tau}-\left(\sigma_{N} L^{2}+\sigma_{m}^{2}\right)\right][} \\
\left.\left(\mu^{2} L^{2}+\sigma_{m}^{2}\right) e^{-i v \tau}-\left(\mu^{2} \sigma_{N} L^{2}+\sigma_{f} \sigma_{m}^{2}\right)\right]+\frac{1}{2} \tau^{2} f^{2} \sigma_{m}^{2}\left(\mu^{2} L^{2}+\sigma_{m}^{2}\right) e^{-i v \tau}= \\
-\mu^{2} \tau^{2}\left(N^{2} L^{2}+f^{2} \sigma_{m}^{2}\right)\left(L^{2}+\sigma_{m}^{2}\right)+\frac{1}{2} \tau^{2} f^{2} \sigma_{m}^{2}\left(\mu^{2} \sigma_{N} L^{2}+\sigma_{f} \sigma_{m}^{2}\right)
\end{array}
$$

Arranging the terms:

$$
\begin{array}{r}
\left(L^{2}+\sigma_{m}^{2}\right)\left(\mu^{2} L^{2}+\sigma_{m}^{2}\right) e^{-2 i v \tau}-\left[\left(L^{2}+\sigma_{m}^{2}\right)\left(\mu^{2} \sigma_{N} L^{2}+\sigma_{f} \sigma_{m}^{2}\right)+\left(\mu^{2} L^{2}+\sigma_{m}^{2}\right)\left(\sigma_{N} L^{2}+\sigma_{f} \sigma_{m}^{2}\right)\right] e^{-i v \tau} \\
+\left(\sigma_{N} L^{2}+\sigma_{f} \sigma_{m}^{2}\right)\left(\mu^{2} \sigma_{N} L^{2}+\sigma_{f} \sigma_{m}^{2}\right)+\mu^{2} \tau^{2}\left(N^{2} L^{2}+f^{2} \sigma_{m}^{2}\right)\left(L^{2}+\sigma_{m}^{2}\right)=0
\end{array}
$$

Rearranging the terms:

$$
\begin{aligned}
e^{-2 i v \tau}-\left[\left(\frac{\mu^{2} \sigma_{N} L^{2}+\sigma_{f} \sigma_{m}^{2}}{\mu^{2} L^{2}+\sigma_{m}^{2}}\right)\right. & \left.+\left(\frac{\sigma_{N} L^{2}+\sigma_{f} \sigma_{m}^{2}}{L^{2}+\sigma_{m}^{2}}\right)\right] e^{-i v \tau} \\
& +\left(\frac{\sigma_{N} L^{2}+\sigma_{f} \sigma_{m}^{2}}{L^{2}+\sigma_{m}^{2}}\right)\left(\frac{\mu^{2} \sigma_{N} L^{2}+\sigma_{f} \sigma_{m}^{2}}{\mu^{2} L^{2}+\sigma_{m}^{2}}\right)+\mu^{2} \tau^{2}\left(\frac{N^{2} L^{2}+f^{2} \sigma_{m}^{2}}{\mu^{2} L^{2}+\sigma_{m}^{2}}\right)=0
\end{aligned}
$$

From (89):

Solution 1:

$$
\begin{aligned}
& e^{v_{i} \tau} \cos \left(v_{r} \tau\right)-1=0 \text { and } e^{v_{i} \tau} \sin \left(v_{r} \tau\right)=0 \\
& v_{r}=0 \quad \text { and } \quad e^{v_{i} \tau}=1
\end{aligned}
$$

Solution 2:

$$
\begin{aligned}
& e^{2 v_{i} \tau} \cos \left(2 v_{r} \tau\right)-\left[\left(\frac{\mu^{2} \sigma_{N} L^{2}+\sigma_{f} \sigma_{m}^{2}}{\mu^{2} L^{2}+\sigma_{m}^{2}}\right)+\left(\frac{\sigma_{N} L^{2}+\sigma_{f} \sigma_{m}^{2}}{L^{2}+\sigma_{m}^{2}}\right)\right] e^{v_{i} \tau} \cos \left(v_{r} \tau\right) \\
&+\left(\frac{\sigma_{N} L^{2}+\sigma_{f} \sigma_{m}^{2}}{L^{2}+\sigma_{m}^{2}}\right)\left(\frac{\mu^{2} \sigma_{N} L^{2}+\sigma_{f} \sigma_{m}^{2}}{\mu^{2} L^{2}+\sigma_{m}^{2}}\right)+\mu^{2} \tau^{2}\left(\frac{N^{2} L^{2}+f^{2} \sigma_{m}^{2}}{\mu^{2} L^{2}+\sigma_{m}^{2}}\right)=0
\end{aligned}
$$




$$
e^{v_{i} \tau}=\left[\left(\frac{\mu^{2} \sigma_{N} L^{2}+\sigma_{f} \sigma_{m}^{2}}{\mu^{2} L^{2}+\sigma_{m}^{2}}\right)+\left(\frac{\sigma_{N} L^{2}+\sigma_{f} \sigma_{m}^{2}}{L^{2}+\sigma_{m}^{2}}\right)\right] \frac{\sin \left(v_{r} \tau\right)}{\sin \left(2 v_{r} \tau\right)}
$$

Balance solution:

Using $\hat{\omega}_{z}^{(\mathrm{n}+1)}-\hat{\omega}_{z}^{(\mathrm{n})}=0$ and $\hat{D}=0$ in (76)

$0=-f \sigma_{m}^{2} \hat{\omega}_{z}^{(\mathrm{n})}+L^{2}\left(\underset{\sim}{i m}-\frac{1}{2 H}\right) \hat{B}^{(\mathrm{n})} \quad$ or $\quad 0=f\left(\underset{\sim}{i m}+\frac{1}{2 H}\right) \hat{\omega}_{z}^{(\mathrm{n})}+L^{2} \hat{B}^{(\mathrm{n})}$

Using $\hat{D}=0$ in (77):

$0=f \sigma_{m}^{2} \hat{\omega}_{z}^{(\mathrm{n})}-L^{2}\left(\underset{\sim}{\operatorname{im}}-\frac{1}{2 H}\right) \hat{B}^{(\mathrm{n})} \quad$ or $\quad 0=f\left(\underset{\sim}{i m}+\frac{1}{2 H}\right) \hat{\omega}_{z}^{(\mathrm{n})}+L^{2} \hat{B}^{(\mathrm{n})}$

Using $\hat{B}^{(\mathrm{n}+1)}-\hat{B}^{(\mathrm{n})}=0$ and $\hat{D}=0$ in (78):

$0=-L^{2}\left(\underset{\sim}{i m}-\frac{1}{2 H}\right) \hat{B}^{(\mathrm{n})}+f \sigma_{m}^{2} \hat{\omega}_{z}^{(\mathrm{n})} \quad$ or $\quad 0=L^{2} \hat{B}^{(\mathrm{n})}+f\left(\underset{\sim}{\underset{i}{m}} m+\frac{1}{2 H}\right) \hat{\boldsymbol{\omega}}_{z}^{(\mathrm{n})}$

Eqs. (92)-(94) yield a unique solution.

Approximating (67) with $\mathrm{w}=0$ (because $\mathrm{D}=0$ ) and using (63):

$$
0=-\left(\underset{\sim}{i} m+\frac{1}{2 H}\right) \hat{P}_{D}+\hat{B}^{(\mathrm{n})}
$$

Approximating(66) and (60) with $\mathrm{w}=0$ and $\mathrm{D}=0$ :

$$
0=\hat{\omega}_{z}^{(\mathrm{n})}+L^{2} \hat{P}_{D}
$$

Using (95) in (92)-(94) yields (96). There is a unique balance solution with Scheme II. 
(a)

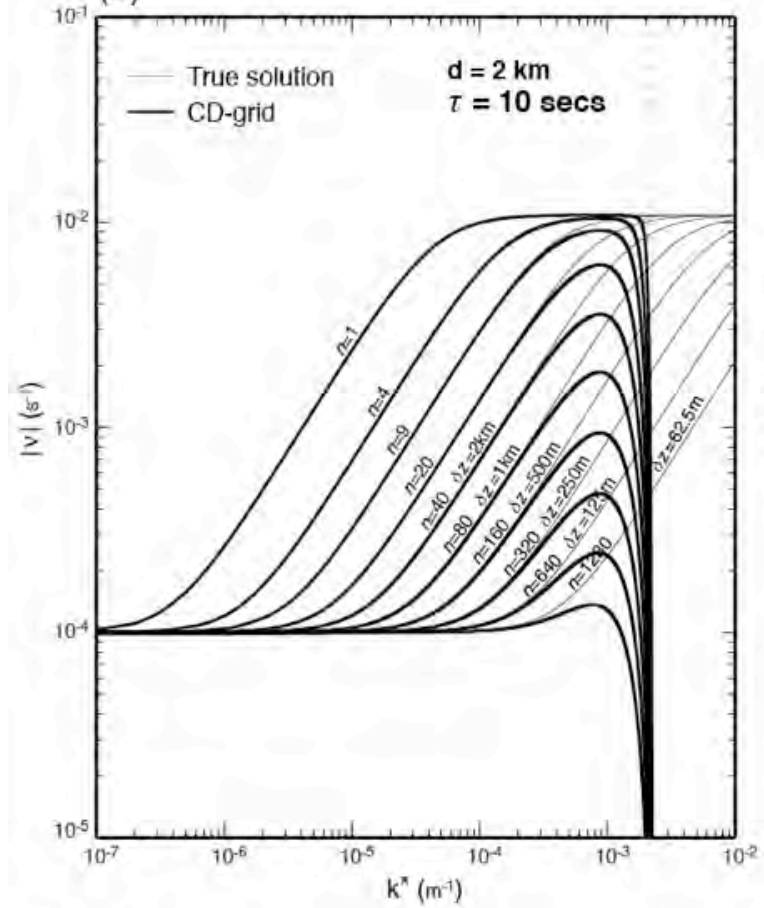

(c)

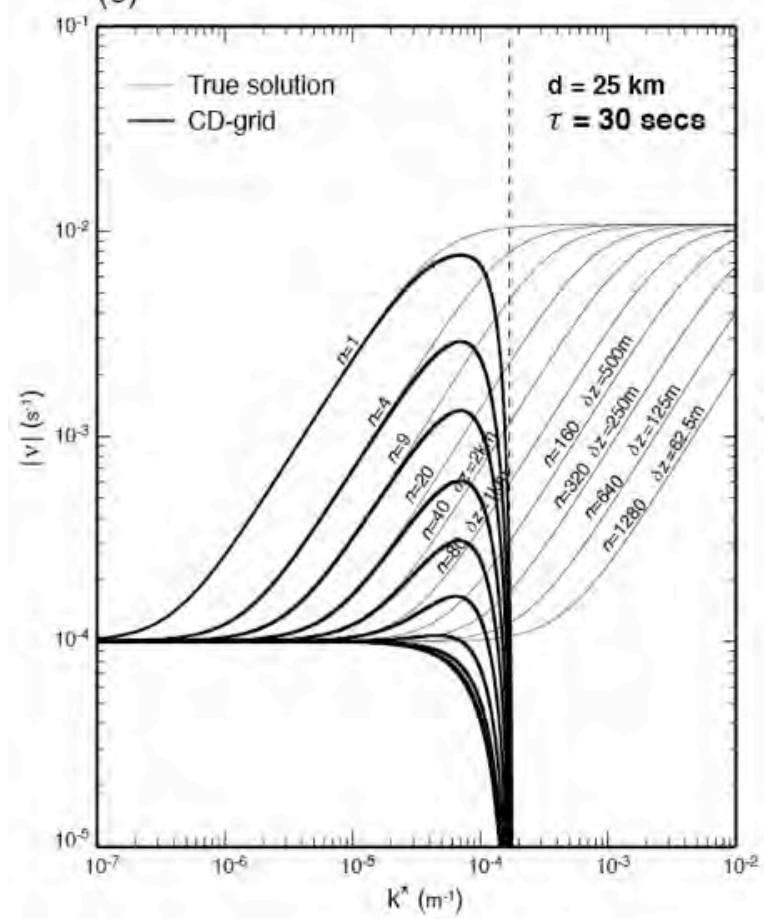

(b)

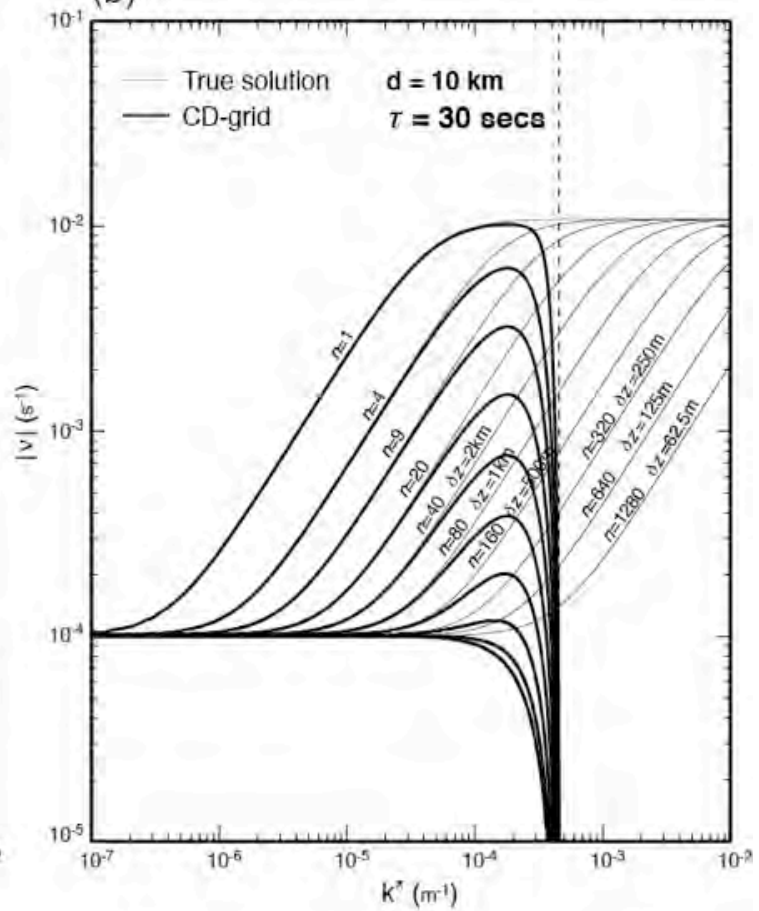

(d)

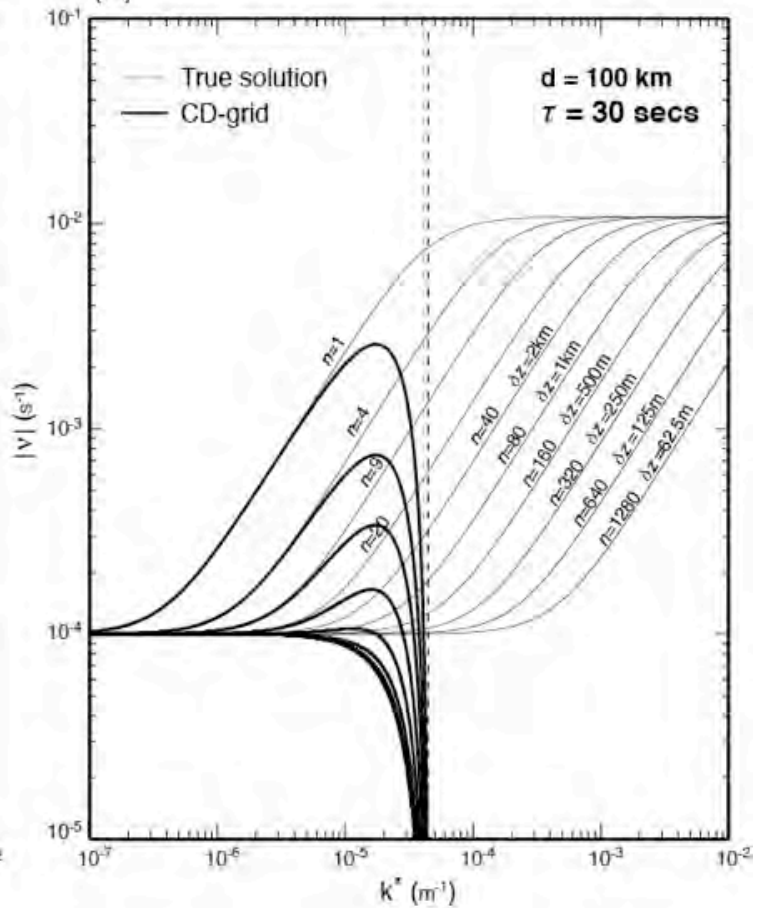

Figure. Scheme II. (runs_CD_grid_time_discrete_scheme_2) 


\section{Scheme III:}

Predictor-step on C grid:

$$
\begin{aligned}
& \hat{\omega}_{z}^{(*)}=\mu \hat{\omega}_{z}^{(\mathrm{n})}-\frac{\tau}{2} f \hat{D}^{(\mathrm{n})} \\
& \hat{D}^{(*)}=\mu \hat{D}^{(\mathrm{n})}+\frac{\tau}{2}\left(f \hat{\omega}_{z}^{(\mathrm{n})}+L^{2} \hat{P}_{C}\right) \\
& \hat{w}^{(*)}=\hat{w}^{(\mathrm{n})}+\frac{\tau}{2}\left[-\left(\underset{\sim}{i} m+\frac{1}{2 H}\right) \hat{P}_{C}+\hat{B}^{(\mathrm{n})}\right] \\
& \hat{B}^{(*)}=\hat{B}^{(\mathrm{n})}-\frac{\tau}{2} N^{2} \hat{w}^{(\mathrm{n})} \\
& \hat{D}^{(*)}+\left(\underset{\sim}{i} m-\frac{1}{2 H}\right) \hat{w}^{(*)}=0
\end{aligned}
$$

\section{Corrector-step on D grid:}

$$
\begin{aligned}
& \hat{\omega}_{z}^{(\mathrm{n}+1)}=\hat{\omega}_{z}^{(\mathrm{n})}-\tau f \hat{D}^{(*)} \\
& \hat{D}^{(\mathrm{n}+1)}=\hat{D}^{(\mathrm{n})}+\tau\left(f \mu \hat{\omega}_{z}^{(\mathrm{n}+1)}+\mu L^{2} \hat{P}_{D}\right) \\
& \hat{w}^{(\mathrm{n}+1)}=\hat{w}^{(\mathrm{n})}+\tau\left[-\left(\underset{\sim}{\underset{\sim}{m}} m+\frac{1}{2 H}\right) \hat{P}_{D}+\hat{B}^{(n+1)}\right] \\
& \hat{B}^{(\mathrm{n}+1)}=\hat{B}^{(\mathrm{n})}-\tau N^{2} \hat{w}^{(*)} \\
& \mu \hat{D}^{(\mathrm{n}+1)}+\left(\underset{\sim}{i m}-\frac{1}{2 H}\right) \hat{w}^{(\mathrm{n}+1)}=0
\end{aligned}
$$

\section{Predictor-step equations:}

Predictor-step is identical to scheme II. Rewriting (71) and (72):

$$
\begin{aligned}
-\left(L^{2}+m^{2}+\frac{1}{4 H^{2}}\right) \hat{D}^{(*)}=-\mu\left(m^{2}+\frac{1}{4 H^{2}}\right) \hat{D}^{(\mathrm{n})} & \\
& -\frac{\tau}{2} f\left(m^{2}+\frac{1}{4 H^{2}}\right) \hat{\omega}_{z}^{(\mathrm{n})}+L^{2}\left(\underset{\sim}{i} m-\frac{1}{2 H}\right) \hat{w}^{(\mathrm{n})}+\frac{\tau}{2}\left(\underset{\sim}{i} m-\frac{1}{2 H}\right) L^{2} \hat{B}^{(\mathrm{n})}
\end{aligned}
$$

and

$$
\begin{aligned}
\left(L^{2}+m^{2}\right. & \left.+\frac{1}{4 H^{2}}\right)\left(\underset{\sim}{i m}-\frac{1}{2 H}\right) \hat{w}^{(*)}=-\mu\left(m^{2}+\frac{1}{4 H^{2}}\right) \hat{D}^{(\mathrm{n})} \\
& -\frac{\tau}{2} f\left(m^{2}+\frac{1}{4 H^{2}}\right) \hat{\boldsymbol{\omega}}_{z}^{(\mathrm{n})}+L^{2}\left(\underset{\sim}{i} m-\frac{1}{2 H}\right) \hat{w}^{(\mathrm{n})}+\frac{\tau}{2}\left(\underset{\sim}{i} m-\frac{1}{2 H}\right) L^{2} \hat{B}^{(\mathrm{n})}
\end{aligned}
$$

Using (129) [ and $\mu \hat{D}^{(\mathrm{n})}+\left(\underset{\sim}{\underset{\sim}{i}}-\frac{1}{2 H}\right) \hat{w}^{(\mathrm{n})}=0$ ] in (130) and (131):

$$
\left(L^{2}+m^{2}+\frac{1}{4 H^{2}}\right) \hat{D}^{(*)}=\mu\left(L^{2}+m^{2}+\frac{1}{4 H^{2}}\right) \hat{D}^{(\mathrm{n})}+\frac{\tau}{2} f\left(m^{2}+\frac{1}{4 H^{2}}\right) \hat{\omega}_{z}^{(\mathrm{n})}-\frac{\tau}{2}\left(\underset{\sim}{i m}-\frac{1}{2 H}\right) L^{2} \hat{B}^{(\mathrm{n})}
$$

and 


$$
\begin{aligned}
\left(L^{2}+m^{2}+\frac{1}{4 H^{2}}\right) & \left(\underset{\sim}{i m}-\frac{1}{2 H}\right) \hat{w}^{(*)}= \\
& -\mu\left(L^{2}+m^{2}+\frac{1}{4 H^{2}}\right) \hat{D}^{(\mathrm{n})}-\frac{\tau}{2} f\left(m^{2}+\frac{1}{4 H^{2}}\right) \hat{\boldsymbol{\omega}}_{z}^{(\mathrm{n})}+\frac{\tau}{2}\left(\underset{\sim}{i m}-\frac{1}{2 H}\right) L^{2} \hat{B}^{(\mathrm{n})}
\end{aligned}
$$

\section{Corrector-step equations:}

Using (132) in (125):

$$
\begin{aligned}
\left(L^{2}+m^{2}+\frac{1}{4 H^{2}}\right)\left(\hat{\omega}_{z}^{(\mathrm{n}+1)}-\hat{\omega}_{z}^{(\mathrm{n})}\right) & +\frac{\tau^{2} f^{2}}{2}\left(m^{2}+\frac{1}{4 H^{2}}\right) \hat{\boldsymbol{\omega}}_{z}^{(\mathrm{n})}= \\
& -\mu \tau f\left(L^{2}+m^{2}+\frac{1}{4 H^{2}}\right) \hat{D}^{(\mathrm{n})}+\frac{\tau^{2}}{2} f\left(\underset{\sim}{i m}-\frac{1}{2 H}\right) L^{2} \hat{B}^{(\mathrm{n})}
\end{aligned}
$$

Eliminating $\hat{P}_{D}$ in (126) and (127):

$$
\left(\underset{\sim}{i m}+\frac{1}{2 H}\right) \hat{D}^{(\mathrm{n}+1)}+\mu L^{2} \hat{w}^{(\mathrm{n}+1)}=\left(\underset{\sim}{i} m+\frac{1}{2 H}\right) \hat{D}^{(\mathrm{n})}+\mu L^{2} \hat{w}^{(\mathrm{n})}+\mu \tau f\left(\underset{\sim}{i} m+\frac{1}{2 H}\right) \hat{\omega}_{z}^{(\mathrm{n}+1)}+\mu L^{2} \tau \hat{B}^{(\mathrm{n}+1)}
$$

Using (129) in (135):

$$
\left(\mu^{2} L^{2}+m^{2}+\frac{1}{4 H^{2}}\right)\left(\hat{D}^{(\mathrm{n}+1)}-\hat{D}^{(\mathrm{n})}\right)=\mu \tau f\left(m^{2}+\frac{1}{4 H^{2}}\right) \hat{\omega}_{z}^{(\mathrm{n}+1)}-\mu L^{2} \tau\left(\underset{\sim}{i} m-\frac{1}{2 H}\right) \hat{B}^{(\mathrm{n}+1)}
$$

Using (133) in (128):

$$
\begin{aligned}
& \left(L^{2}+m^{2}+\frac{1}{4 H^{2}}\right)\left(\underset{\sim}{i m}-\frac{1}{2 H}\right)\left(\hat{B}^{(\mathrm{n}+1)}-\hat{B}^{(\mathrm{n})}\right)+\frac{\tau^{2} N^{2}}{2}\left(\underset{\sim}{\operatorname{im}}-\frac{1}{2 H}\right) L^{2} \hat{B}^{(\mathrm{n})}= \\
& \mu \tau N^{2}\left(L^{2}+m^{2}+\frac{1}{4 H^{2}}\right) \hat{D}^{(\mathrm{n})}+\frac{\tau^{2} N^{2}}{2} f\left(m^{2}+\frac{1}{4 H^{2}}\right) \hat{\boldsymbol{\omega}}_{z}^{(\mathrm{n})}
\end{aligned}
$$

Eqs. (134), (136) and (137) are the equations of the system.

Using $\hat{\Phi}^{(\mathrm{n}+1)}=e^{-i v \tau} \hat{\Phi}^{(\mathrm{n})}$ in (134), (136) and (137):

$$
\left[\left(L^{2}+m^{2}+\frac{1}{4 H^{2}}\right)\left(e^{-i v \tau}-1\right)+\frac{\tau^{2} f^{2}}{2}\left(m^{2}+\frac{1}{4 H^{2}}\right)\right] \hat{\omega}_{z}=-\mu \tau f\left(L^{2}+m^{2}+\frac{1}{4 H^{2}}\right) \hat{D}+\frac{\tau^{2}}{2} f\left(\underset{\sim}{i} m-\frac{1}{2 H}\right) L^{2} \hat{B}
$$




$$
\begin{gathered}
\left(\mu^{2} L^{2}+m^{2}+\frac{1}{4 H^{2}}\right)\left(e^{-i v \tau}-1\right) \hat{D}=\mu \tau f\left(m^{2}+\frac{1}{4 H^{2}}\right) e^{-i v \tau} \hat{\omega}_{z}-\mu L^{2} \tau\left(\underset{\sim}{i} m-\frac{1}{2 H}\right) e^{-i v \tau} \hat{B} \\
{\left[\left(L^{2}+m^{2}+\frac{1}{4 H^{2}}\right)\left(e^{-i v \tau}-1\right)+\frac{\tau^{2} N^{2}}{2} L^{2}\right]\left(\underset{\sim}{i} m-\frac{1}{2 H}\right) \hat{B}=} \\
\mu \tau N^{2}\left(L^{2}+m^{2}+\frac{1}{4 H^{2}}\right) \hat{D}+\frac{\tau^{2} N^{2}}{2} f\left(m^{2}+\frac{1}{4 H^{2}}\right) \hat{\omega}_{z}
\end{gathered}
$$

Eliminating D between (138) and (140):

$$
\left(e^{-i v \tau}-1\right)\left(L^{2}+m^{2}+\frac{1}{4 H^{2}}\right)\left[N^{2} \hat{\omega}_{z}+f\left(\underset{\sim}{i} m-\frac{1}{2 H}\right) \hat{B}\right]=0
$$

Requiring

$$
\left(e^{-i v \tau}-1\right)\left(L^{2}+m^{2}+\frac{1}{4 H^{2}}\right) \neq 0
$$

Then rewriting (141):

$$
N^{2} \hat{\omega}_{z}+f\left(\underset{\sim}{i} m-\frac{1}{2 H}\right) \hat{B}=0
$$

Eliminating $D$ between (138) and (139) and $\sigma_{m}^{2} \equiv m^{2}+\frac{1}{4 H^{2}}$ :

$$
\begin{array}{r}
\left\{\left(\mu^{2} L^{2}+\sigma_{m}^{2}\right)\left(e^{-i v \tau}-1\right)\left[\left(L^{2}+\sigma_{m}^{2}\right)\left(e^{-i v \tau}-1\right)+\frac{\tau^{2} f^{2}}{2} \sigma_{m}^{2}\right]+\mu^{2} \tau^{2} f^{2}\left(L^{2}+\sigma_{m}^{2}\right) \sigma_{m}^{2} e^{-i v \tau}\right\} \hat{\omega}_{z}= \\
\tau^{2} f\left[\mu^{2}\left(L^{2}+\sigma_{m}^{2}\right) e^{-i v \tau}+\frac{1}{2}\left(\mu^{2} L^{2}+\sigma_{m}^{2}\right)\left(e^{-i v \tau}-1\right)\right]\left(\underset{\sim}{i m}-\frac{1}{2 H}\right) L^{2} \hat{B}
\end{array}
$$

Eliminating B between (144) and (143):

$$
\begin{array}{r}
\left\{\left(\mu^{2} L^{2}+\sigma_{m}^{2}\right)\left(e^{-i v \tau}-1\right)\left[\left(L^{2}+\sigma_{m}^{2}\right)\left(e^{-i v \tau}-1\right)+\frac{\tau^{2} f^{2}}{2} \sigma_{m}^{2}\right]+\mu^{2} \tau^{2} f^{2}\left(L^{2}+\sigma_{m}^{2}\right) \sigma_{m}^{2} e^{-i v \tau}\right\} \hat{\boldsymbol{\omega}}_{z}= \\
-\tau^{2}\left[\mu^{2}\left(L^{2}+\sigma_{m}^{2}\right) e^{-i v \tau}+\frac{1}{2}\left(\mu^{2} L^{2}+\sigma_{m}^{2}\right)\left(e^{-i v \tau}-1\right)\right] N^{2} L^{2} \hat{\boldsymbol{\omega}}_{z}
\end{array}
$$


Nontrivial solutions of (145):

$\left(\mu^{2} L^{2}+\sigma_{m}^{2}\right)\left(e^{-i v \tau}-1\right)\left[\left(L^{2}+\sigma_{m}^{2}\right)\left(e^{-i v \tau}-1\right)+\frac{\tau^{2} f^{2}}{2} \sigma_{m}^{2}+\frac{\tau^{2} N^{2}}{2} L^{2}\right]+\mu^{2} \tau^{2}\left(L^{2}+\sigma_{m}^{2}\right)\left(N^{2} L^{2}+f^{2} \sigma_{m}^{2}\right) e^{-i v \tau}=0$

Arranging the terms in (146) and using $\sigma_{f} \equiv 1-\frac{1}{2} \tau^{2} f^{2}$ and $\sigma_{N} \equiv 1-\frac{1}{2} \tau^{2} N^{2}$ :

$$
\begin{aligned}
& \left(L^{2}+\sigma_{m}^{2}\right)\left(\mu^{2} L^{2}+\sigma_{m}^{2}\right) e^{-2 i v \tau} \\
& +\left\{\mu^{2} \tau^{2}\left(L^{2}+\sigma_{m}^{2}\right)\left(N^{2} L^{2}+f^{2} \sigma_{m}^{2}\right)-\left(\mu^{2} L^{2}+\sigma_{m}^{2}\right)\left[\left(L^{2}+\sigma_{m}^{2}\right)+\left(\sigma_{N} L^{2}+\sigma_{f} \sigma_{m}^{2}\right)\right]\right\} e^{-i v \tau} \\
& +\left(\mu^{2} L^{2}+\sigma_{m}^{2}\right)\left(\sigma_{N} L^{2}+\sigma_{f} \sigma_{m}^{2}\right)=0
\end{aligned}
$$

Using $v \equiv v_{r}+\underset{\sim}{i} v_{i}$ in (147) and splitting real and imaginary parts:

$$
\begin{gathered}
\left(L^{2}+\sigma_{m}^{2}\right)\left(\mu^{2} L^{2}+\sigma_{m}^{2}\right) e^{2 v_{i} \tau} \cos \left(2 v_{r} \tau\right) \\
+\left\{\mu^{2} \tau^{2}\left(L^{2}+\sigma_{m}^{2}\right)\left(N^{2} L^{2}+f^{2} \sigma_{m}^{2}\right)-\left(\mu^{2} L^{2}+\sigma_{m}^{2}\right)\left[\left(L^{2}+\sigma_{m}^{2}\right)+\left(\sigma_{N} L^{2}+\sigma_{f} \sigma_{m}^{2}\right)\right]\right\} e^{v_{i} \tau} \cos \left(v_{r} \tau\right) \\
+\left(\mu^{2} L^{2}+\sigma_{m}^{2}\right)\left(\sigma_{N} L^{2}+\sigma_{f} \sigma_{m}^{2}\right)=0
\end{gathered}
$$

and

$$
e^{v_{i} \tau}=\frac{-\left\{\mu^{2} \tau^{2}\left(L^{2}+\sigma_{m}^{2}\right)\left(N^{2} L^{2}+f^{2} \sigma_{m}^{2}\right)-\left(\mu^{2} L^{2}+\sigma_{m}^{2}\right)\left[\left(L^{2}+\sigma_{m}^{2}\right)+\left(\sigma_{N} L^{2}+\sigma_{f} \sigma_{m}^{2}\right)\right]\right\}}{\left(L^{2}+\sigma_{m}^{2}\right)\left(\mu^{2} L^{2}+\sigma_{m}^{2}\right)} \frac{\sin \left(v_{r} \tau\right)}{\sin \left(2 v_{r} \tau\right)}
$$

\section{Balance solution:}

Writing (134), (136) and (137) with $\hat{\omega}_{z}^{(\mathrm{n}+1)}-\hat{\omega}_{z}^{(\mathrm{n})}=0, D^{(\mathrm{n}+1)}=D^{(\mathrm{n})}=0 \quad(\mathrm{w}=0)$ and $\hat{B}^{(\mathrm{n}+1)}-\hat{B}^{(\mathrm{n})}=0$ :

$$
\begin{gathered}
-f\left(\underset{\sim}{i} m+\frac{1}{2 H}\right) \hat{\omega}_{z}^{(\mathrm{n})}=L^{2} \hat{B}^{(\mathrm{n})} \\
0=-f\left(\underset{\sim}{\operatorname{i}} m+\frac{1}{2 H}\right) \hat{\omega}_{z}^{(\mathrm{n}+1)}-L^{2} \hat{B}^{(\mathrm{n}+1)} \\
\left(\underset{\sim}{\operatorname{i}} m-\frac{1}{2 H}\right) L^{2} \hat{B}^{(\mathrm{n})}=-f\left(\underset{\sim}{i} m+\frac{1}{2 H}\right) \hat{\omega}_{z}^{(\mathrm{n})}
\end{gathered}
$$




\section{From (127):}

$$
0=-\left(\underset{\sim}{i} m+\frac{1}{2 H}\right) \hat{P}_{D}+\hat{B}^{(\mathrm{n}+1)}
$$

From (126):

$$
f \hat{\omega}_{z}^{(\mathrm{n}+1)}+L^{2} \hat{P}_{D}=0
$$

Eqs. (153) with (154) satisfies (150)-(152). There is a unique balance solution with scheme III. 
(a)

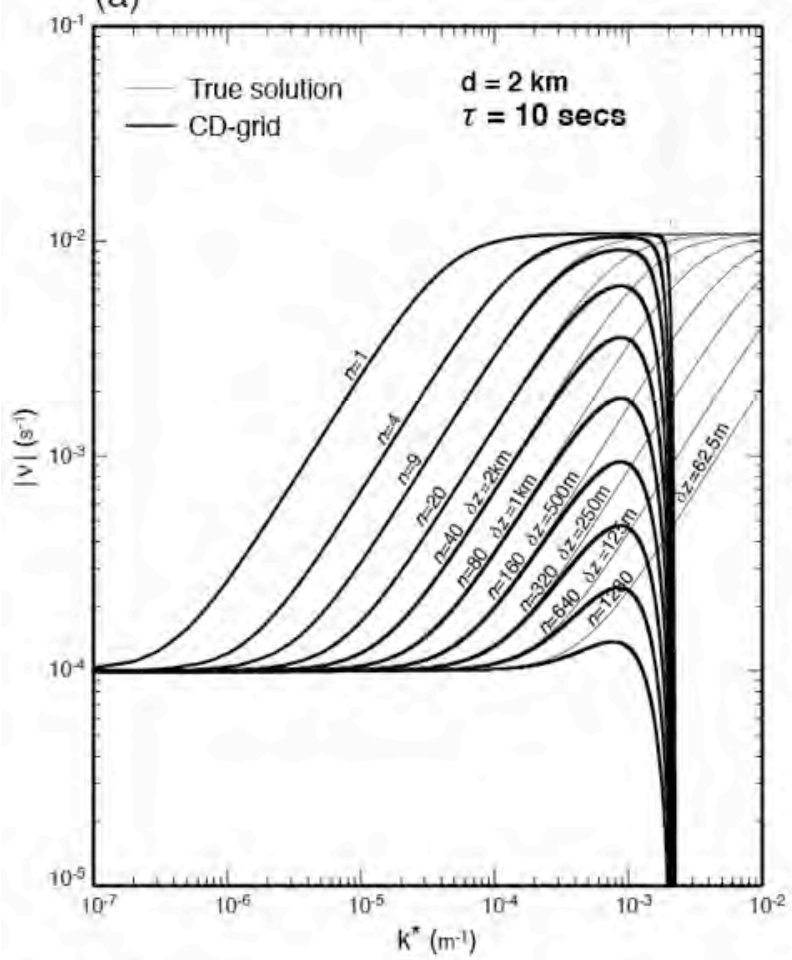

(c)

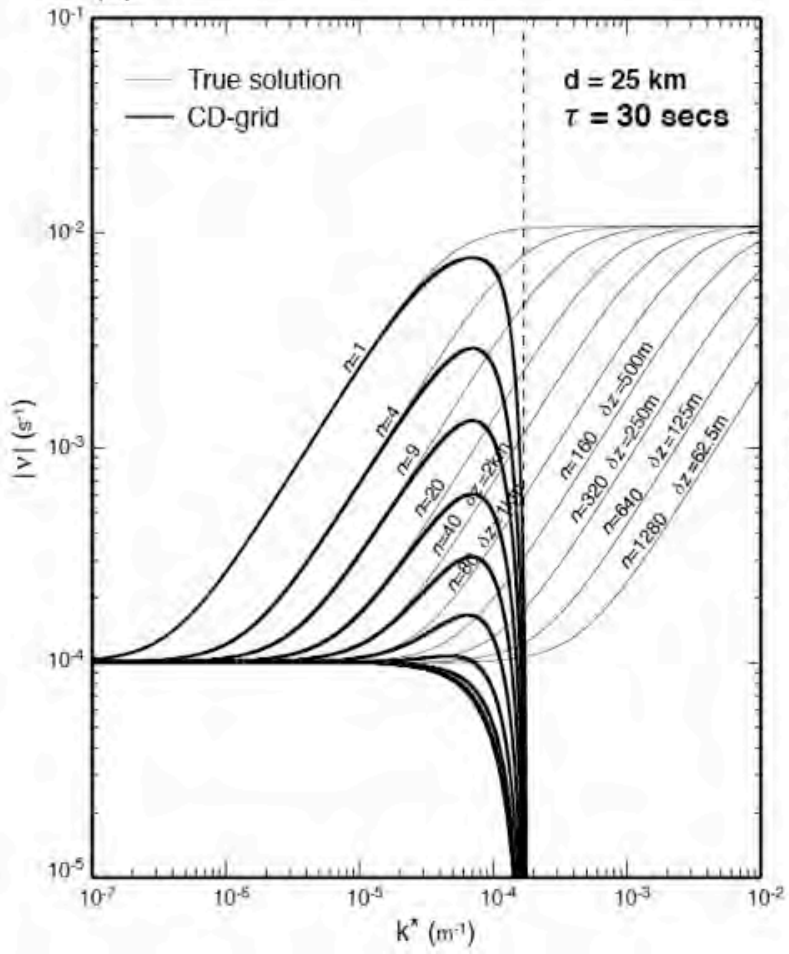

(b)

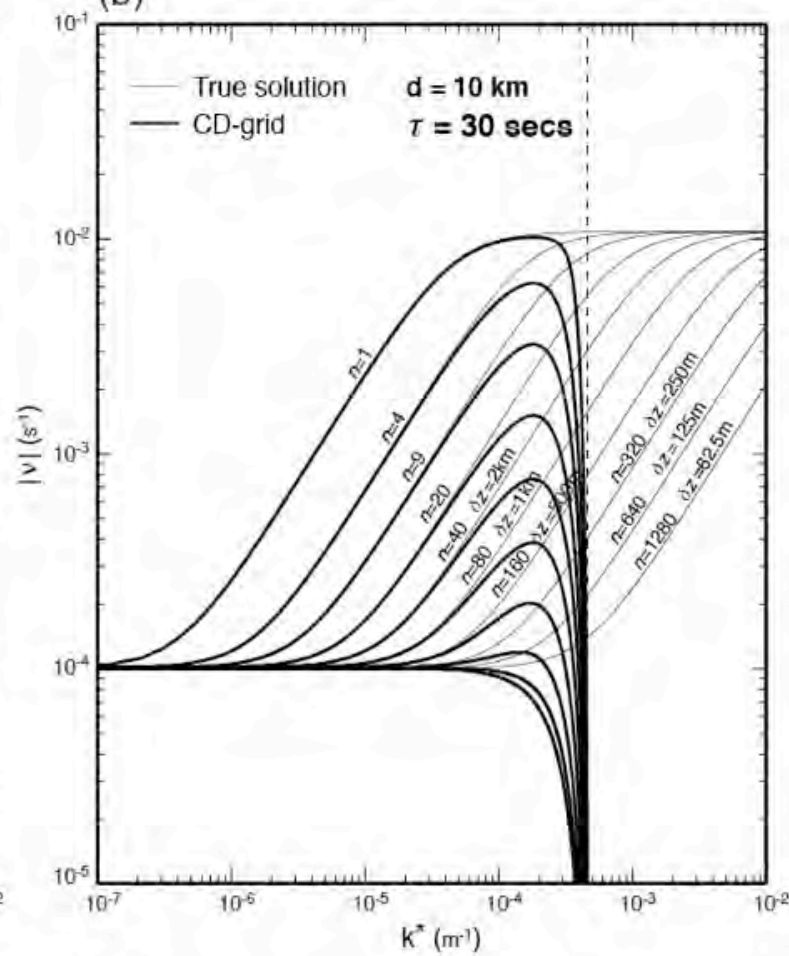

(d)

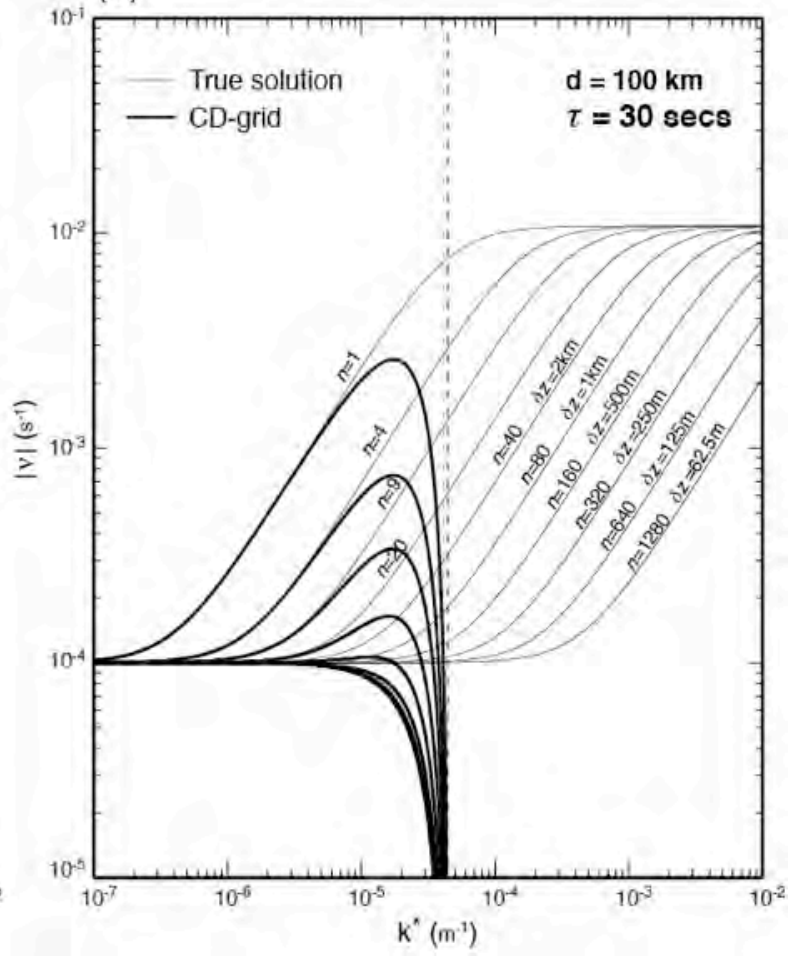

Figure. Scheme III. (runs_CD_grid_time_discrete_scheme_3) 


\section{Scheme IV:}

Predictor-step on the $\mathrm{C}$ grid:

$$
\begin{aligned}
& \hat{\omega}_{z}^{(*)}=\mu \hat{\omega}_{z}^{(\mathrm{n})}-\frac{\tau}{2} f \hat{D}^{(\mathrm{n})} \\
& \hat{D}^{(*)}=\mu \hat{D}^{(\mathrm{n})}+\frac{\tau}{2}\left(f \hat{\omega}_{z}^{(\mathrm{n})}+L^{2} \hat{P}_{C}\right) \\
& \hat{w}^{(*)}=\hat{w}^{(\mathrm{n})}+\frac{\tau}{2}\left[-\left(\underset{\sim}{\operatorname{im}}+\frac{1}{2 H}\right) \hat{P}_{C}+\hat{B}^{(*)}\right] \\
& \hat{B}^{(*)}=\hat{B}^{(\mathrm{n})}-\frac{\tau}{2} N^{2} \hat{w}^{(*)} \\
& \hat{D}^{(*)}+\left(\underset{\sim}{i m}-\frac{1}{2 H}\right) \hat{w}^{(*)}=0
\end{aligned}
$$

\section{Corrector-step on the D grid:}

$$
\begin{aligned}
& \hat{\omega}_{z}^{(\mathrm{n}+1)}=\hat{\omega}_{z}^{(\mathrm{n})}-\tau f \hat{D}^{(*)}(175) \\
& \hat{D}^{(\mathrm{n}+1)}=\hat{D}^{(\mathrm{n})}+\tau\left(f \hat{\omega}_{z}^{(*)}+\mu L^{2} \hat{P}_{D}\right)(176) \\
& \hat{w}^{(\mathrm{n}+1)}=\hat{w}^{(\mathrm{n})}+\tau\left[-\left({\underset{\sim}{i}}_{\sim}+\frac{1}{2 H}\right) \hat{P}_{D}+\hat{B}^{(\mathrm{n}+1)}\right] \\
& \hat{B}^{(\mathrm{n}+1)}=\hat{B}^{(\mathrm{n})}-\tau N^{2} \hat{w}^{(\mathrm{n}+1)}(178) \\
& \mu \hat{D}^{(\mathrm{n}+1)}+\left(\underset{\sim}{i m}-\frac{1}{2 H}\right) \hat{w}^{(\mathrm{n}+1)}=0
\end{aligned}
$$

Predictor-step equations:

Eliminating $\hat{P}_{C}$ between (171) and (172) and using (173), (174) and $\mu \hat{D}^{(\mathrm{n})}+\left(\underset{\sim}{\operatorname{im}}-\frac{1}{2 H}\right) \hat{w}^{(\mathrm{n})}=0$ :

$$
\left[\left(1+\frac{\tau^{2} N^{2}}{4}\right) L^{2}+m^{2}+\frac{1}{4 H^{2}}\right] \hat{D}^{(*)}=\mu\left(L^{2}+m^{2}+\frac{1}{4 H^{2}}\right) \hat{D}^{(\mathrm{n})}+\frac{\tau}{2} f\left(m^{2}+\frac{1}{4 H^{2}}\right) \hat{\omega}_{z}^{(\mathrm{n})}-\frac{\tau}{2}\left(\underset{\sim}{i m}-\frac{1}{2 H}\right) L^{2} \hat{B}^{(\mathrm{n})}
$$

\section{Corrector-step equations:}

Using (180) in (175):

$$
\begin{aligned}
{\left[\left(1+\frac{\tau^{2} N^{2}}{4}\right) L^{2}+m^{2}+\frac{1}{4 H^{2}}\right]\left(\hat{\omega}_{z}^{(\mathrm{n}+1)}-\right.} & \left.\hat{\omega}_{z}^{(\mathrm{n})}\right)+\frac{\tau^{2} f^{2}}{2}\left(m^{2}+\frac{1}{4 H^{2}}\right) \hat{\omega}_{z}^{(\mathrm{n})}= \\
& -\tau f \mu\left(L^{2}+m^{2}+\frac{1}{4 H^{2}}\right) \hat{D}^{(\mathrm{n})}+\frac{\tau^{2}}{2} f\left(\underset{\sim}{i m}-\frac{1}{2 H}\right) L^{2} \hat{B}^{(\mathrm{n})}
\end{aligned}
$$

Eliminating $\hat{P}_{D}$ between (176) and (177) and using (170), (178) and (179): 


$$
\begin{aligned}
{\left[\mu^{2}\left(1+\tau^{2} N^{2}\right) L^{2}+\left(m^{2}+\frac{1}{4 H^{2}}\right)\right] \hat{D}^{(\mathrm{n}+1)}=} & {\left[\mu^{2} L^{2}+\left(1-\frac{\tau^{2} f^{2}}{2}\right)\left(m^{2}+\frac{1}{4 H^{2}}\right)\right] \hat{D}^{(\mathrm{n})} } \\
& +\mu \tau f\left(m^{2}+\frac{1}{4 H^{2}}\right) \hat{\omega}_{z}^{(\mathrm{n})}-\mu \tau\left(\underset{\sim}{\underset{i}{m}}-\frac{1}{2 H}\right) L^{2} \hat{B}^{(\mathrm{n})}
\end{aligned}
$$

Using (179) in (178):

$$
\left(\underset{\sim}{i m}-\frac{1}{2 H}\right)\left(\hat{B}^{(\mathrm{n}+1)}-\hat{B}^{(\mathrm{n})}\right)=\mu \tau N^{2} \hat{D}^{(\mathrm{n}+1)}
$$

Eqs. (181), (182) and (183) are the equations of the system.

Using $\hat{\Phi}^{(\mathrm{n}+1)}=e^{-i v \tau} \hat{\Phi}^{(\mathrm{n})}$ in (181):

$$
\begin{aligned}
& {\left[\left(1+\frac{\tau^{2} N^{2}}{4}\right) L^{2}+m^{2}+\frac{1}{4 H^{2}}\right]\left(e^{-i v \tau}-1\right) \hat{\omega}_{z}+\frac{\tau^{2} f^{2}}{2}\left(m^{2}+\frac{1}{4 H^{2}}\right) \hat{\omega}_{z}=} \\
& \quad-\tau f \mu\left(L^{2}+m^{2}+\frac{1}{4 H^{2}}\right) \hat{D}+\frac{\tau^{2}}{2} f\left(\underset{\sim}{i m}-\frac{1}{2 H}\right) L^{2} \hat{B}
\end{aligned}
$$

Using $\hat{\Phi}^{(\mathrm{n}+1)}=e^{-i v \tau} \hat{\Phi}^{(\mathrm{n})}$ in (182):

$$
\begin{aligned}
{\left[\mu^{2}\left(1+\tau^{2} N^{2}\right) L^{2}+\left(m^{2}+\frac{1}{4 H^{2}}\right)\right] e^{-i v \tau} \hat{D}=} & {\left[\mu^{2} L^{2}+\left(1-\frac{\tau^{2} f^{2}}{2}\right)\left(m^{2}+\frac{1}{4 H^{2}}\right)\right] \hat{D} } \\
& +\mu \tau f\left(m^{2}+\frac{1}{4 H^{2}}\right) \hat{\omega}_{z}-\mu \tau\left(\underset{\sim}{i m-} \frac{1}{2 H}\right) L^{2} \hat{B}
\end{aligned}
$$

Using $\hat{\Phi}^{(\mathrm{n}+1)}=e^{-i v \tau} \hat{\Phi}^{(\mathrm{n})}$ in (183):

$$
\left(e^{-i v \tau}-1\right)\left(\underset{\sim}{i} m-\frac{1}{2 H}\right) \hat{B}=\mu \tau N^{2} e^{-i v \tau} \hat{D}
$$

Eliminating B between (184) and (186):

$$
\begin{aligned}
\left(e^{-i v \tau}-1\right)\left\{\left[\left(1+\frac{\tau^{2} N^{2}}{4}\right) L^{2}+m^{2}+\frac{1}{4 H^{2}}\right]\right. & \left.\left(e^{-i v \tau}-1\right)+\frac{\tau^{2} f^{2}}{2}\left(m^{2}+\frac{1}{4 H^{2}}\right)\right\} \hat{\omega}_{z}= \\
& -\tau f \mu\left[\left(e^{-i v \tau}-1\right)\left(L^{2}+m^{2}+\frac{1}{4 H^{2}}\right)-\frac{\tau^{2} N^{2}}{2} L^{2} e^{-i v \tau}\right] \hat{D}
\end{aligned}
$$

Eliminating B between (184) and (187): 


$$
\begin{gathered}
{\left[\mu^{2}\left(1+\tau^{2} N^{2}\right) L^{2}+\left(m^{2}+\frac{1}{4 H^{2}}\right)\right]\left(e^{-i v \tau}-1\right) e^{-i v \tau} \hat{D}=\left[\mu^{2} L^{2}+\left(1-\frac{\tau^{2} f^{2}}{2}\right)\left(m^{2}+\frac{1}{4 H^{2}}\right)\right]\left(e^{-i v \tau}-1\right) \hat{D}} \\
-\mu^{2} \tau^{2} N^{2} L^{2} e^{-i v \tau} \hat{D}+\mu \tau f\left(e^{-i v \tau}-1\right)\left(m^{2}+\frac{1}{4 H^{2}}\right) \hat{\omega}_{z}
\end{gathered}
$$

Rewriting (187) and (188) using $\sigma_{N}^{(+4)} \equiv 1+\frac{\tau^{2} N^{2}}{4}$ and $\sigma_{N}^{(+1)} \equiv 1+\tau^{2} N^{2}$ and definitions:

$a_{1} \equiv \sigma_{N}^{(+4)} L^{2}+\sigma_{m}^{2} ; \quad a_{2} \equiv \sigma_{N} L^{2}+\sigma_{m}^{2} ; \quad a_{3} \equiv L^{2}+\sigma_{m}^{2} ; \quad a_{4} \equiv \mu^{2} \sigma_{N}^{(+1)} L^{2}+\sigma_{m}^{2} ; \quad a_{5} \equiv \mu^{2} L^{2}+\sigma_{f} \sigma_{m}^{2}$

$\left[a_{1}\left(e^{-i v \tau}-1\right)+\left(1-\sigma_{f}\right) \sigma_{m}^{2}\right]\left(e^{-i v \tau}-1\right) \hat{\omega}_{z}=-\tau f \mu\left(a_{2} e^{-i v \tau}-a_{3}\right) \hat{D}$

and

$\left[\left(a_{4} e^{-i v \tau}-a_{5}\right)\left(e^{-i v \tau}-1\right)+\mu^{2} \tau^{2} N^{2} L^{2} e^{-i v \tau}\right] \hat{D}=\mu \tau f\left(e^{-i v \tau}-1\right) \sigma_{m}^{2} \hat{\omega}_{z}$

Eliminating $\hat{\omega}_{z}$ between (189) and (190):

$$
\begin{aligned}
{\left[a_{1}\left(e^{-i v \tau}-1\right)+\left(1-\sigma_{f}\right) \sigma_{m}^{2}\right]\left[\left(a_{4} e^{-i v \tau}-a_{5}\right)\left(e^{-i v \tau}-1\right)\right.} & \left.+\mu^{2} \tau^{2} N^{2} L^{2} e^{-i v \tau}\right] \hat{D}= \\
& -\mu^{2} \tau^{2} f^{2}\left(a_{2} e^{-i v \tau}-a_{3}\right) \sigma_{m}^{2} \hat{D}
\end{aligned}
$$

Arranging the terms:

$$
\begin{aligned}
a_{1}\left(e^{-i v \tau}-1\right)\left[\left(a_{4} e^{-i v \tau}-a_{5}\right)\left(e^{-i v \tau}-1\right)+\mu^{2} \tau^{2} N^{2} L^{2} e^{-i v \tau}\right] & \\
+\left(1-\sigma_{f}\right) \sigma_{m}^{2}\left[\left(a_{4} e^{-i v \tau}-a_{5}\right)\left(e^{-i v \tau}-1\right)\right. & \left.+\mu^{2} \tau^{2} N^{2} L^{2} e^{-i v \tau}\right]= \\
& -\mu^{2} \tau^{2} f^{2} a_{2} e^{-i v \tau} \sigma_{m}^{2}+\mu^{2} \tau^{2} f^{2} a_{3} \sigma_{m}^{2}
\end{aligned}
$$

Rearranging the terms:

$$
\begin{aligned}
a_{1}\left(a_{4} e^{-i v \tau}-a_{5}\right)\left(e^{-i v \tau}-1\right)^{2}+a_{1} \mu^{2} \tau^{2} N^{2} L^{2}\left(e^{-i v \tau}-1\right) e^{-i v \tau} & \\
+\left(1-\sigma_{f}\right) \sigma_{m}^{2}\left(a_{4} e^{-i v \tau}-a_{5}\right)\left(e^{-i v \tau}-1\right) & +\left(1-\sigma_{f}\right) \sigma_{m}^{2} \mu^{2} \tau^{2} N^{2} L^{2} e^{-i v \tau}= \\
& -\mu^{2} \tau^{2} f^{2} a_{2} e^{-i v \tau} \sigma_{m}^{2}+\mu^{2} \tau^{2} f^{2} a_{3} \sigma_{m}^{2}
\end{aligned}
$$

Rearranging the terms: 


$$
\begin{gathered}
a_{1} a_{4} e^{-i v \tau}\left(e^{-i v \tau}-1\right)^{2}-a_{1} a_{5}\left(e^{-i v \tau}-1\right)^{2}+a_{1} \mu^{2} \tau^{2} N^{2} L^{2} e^{-2 i v \tau}-a_{1} \mu^{2} \tau^{2} N^{2} L^{2} e^{-i v \tau} \\
+\left(1-\sigma_{f}\right) \sigma_{m}^{2}\left(a_{4} e^{-i v \tau}-a_{5}\right) e^{-i v \tau}-\left(1-\sigma_{f}\right) \sigma_{m}^{2}\left(a_{4} e^{-i v \tau}-a_{5}\right)+\left(1-\sigma_{f}\right) \sigma_{m}^{2} \mu^{2} \tau^{2} N^{2} L^{2} e^{-i v \tau}= \\
-\mu^{2} \tau^{2} f^{2} a_{2} e^{-i v \tau} \sigma_{m}^{2}+\mu^{2} \tau^{2} f^{2} a_{3} \sigma_{m}^{2}
\end{gathered}
$$

Rearranging the terms:

$$
\begin{aligned}
a_{1} a_{4} e^{-i v \tau}\left(e^{-i v \tau}-1\right)^{2}-a_{1} a_{5}\left(e^{-i v \tau}-1\right)^{2} & +a_{1} \mu^{2} \tau^{2} N^{2} L^{2} e^{-2 i v \tau}-a_{1} \mu^{2} \tau^{2} N^{2} L^{2} e^{-i v \tau} \\
+\left(1-\sigma_{f}\right) \sigma_{m}^{2}\left(a_{4} e^{-i v \tau}-a_{5}\right) e^{-i v \tau}- & a_{4}\left(1-\sigma_{f}\right) \sigma_{m}^{2} e^{-i v \tau}+\left(1-\sigma_{f}\right) \sigma_{m}^{2} \mu^{2} \tau^{2} N^{2} L^{2} e^{-i v \tau}= \\
& -\mu^{2} \tau^{2} f^{2} a_{2} e^{-i v \tau} \sigma_{m}^{2}+\mu^{2} \tau^{2} f^{2} a_{3} \sigma_{m}^{2}-a_{5}\left(1-\sigma_{f}\right) \sigma_{m}^{2}
\end{aligned}
$$

Rearranging the terms:

$$
\begin{gathered}
a_{1} a_{4} e^{-i v \tau}\left(e^{-i v \tau}-1\right)^{2}-a_{1} a_{5}\left(e^{-i v \tau}-1\right)^{2}+a_{1} \mu^{2} \tau^{2} N^{2} L^{2} e^{-2 i v \tau}-a_{1} \mu^{2} \tau^{2} N^{2} L^{2} e^{-i v \tau} \\
+a_{4}\left(1-\sigma_{f}\right) \sigma_{m}^{2} e^{-2 i v \tau}-a_{5}\left(1-\sigma_{f}\right) \sigma_{m}^{2} e^{-i v \tau}-a_{4}\left(1-\sigma_{f}\right) \sigma_{m}^{2} e^{-i v \tau}+\left(1-\sigma_{f}\right) \sigma_{m}^{2} \mu^{2} \tau^{2} N^{2} L^{2} e^{-i v \tau}= \\
-\mu^{2} \tau^{2} f^{2} a_{2} e^{-i v \tau} \sigma_{m}^{2}+\mu^{2} \tau^{2} f^{2} a_{3} \sigma_{m}^{2}-a_{5}\left(1-\sigma_{f}\right) \sigma_{m}^{2}
\end{gathered}
$$

Rearranging the terms:

$$
\begin{aligned}
& a_{1} a_{4} e^{-i v \tau}\left(e^{-i v \tau}-1\right)^{2}-a_{1} a_{5}\left(e^{-i v \tau}-1\right)^{2}+a_{1} \mu^{2} \tau^{2} N^{2} L^{2} e^{-2 i v \tau}+a_{4}\left(1-\sigma_{f}\right) \sigma_{m}^{2} e^{-2 i v \tau} \\
& -a_{1} \mu^{2} \tau^{2} N^{2} L^{2} e^{-i v \tau}-a_{5}\left(1-\sigma_{f}\right) \sigma_{m}^{2} e^{-i v \tau}-a_{4}\left(1-\sigma_{f}\right) \sigma_{m}^{2} e^{-i v \tau} \\
& +\left(1-\sigma_{f}\right) \sigma_{m}^{2} \mu^{2} \tau^{2} N^{2} L^{2} e^{-i v \tau}+\mu^{2} \tau^{2} f^{2} a_{2} \sigma_{m}^{2} e^{-i v \tau}=\mu^{2} \tau^{2} f^{2} a_{3} \sigma_{m}^{2}-a_{5}\left(1-\sigma_{f}\right) \sigma_{m}^{2}
\end{aligned}
$$

Rearranging the terms:

$$
\begin{gathered}
a_{1} a_{4} e^{-i v \tau}\left(e^{-2 i v \tau}-2 e^{-i v \tau}+1\right)-a_{1} a_{5}\left(e^{-2 i v \tau}-2 e^{-i v \tau}+1\right)+a_{1} \mu^{2} \tau^{2} N^{2} L^{2} e^{-2 i v \tau}+a_{4}\left(1-\sigma_{f}\right) \sigma_{m}^{2} e^{-2 i v \tau} \\
-a_{1} \mu^{2} \tau^{2} N^{2} L^{2} e^{-i v \tau}-a_{5}\left(1-\sigma_{f}\right) \sigma_{m}^{2} e^{-i v \tau}-a_{4}\left(1-\sigma_{f}\right) \sigma_{m}^{2} e^{-i v \tau} \\
+\left(1-\sigma_{f}\right) \sigma_{m}^{2} \mu^{2} \tau^{2} N^{2} L^{2} e^{-i v \tau}+\mu^{2} \tau^{2} f^{2} a_{2} \sigma_{m}^{2} e^{-i v \tau}=\mu^{2} \tau^{2} f^{2} a_{3} \sigma_{m}^{2}-a_{5}\left(1-\sigma_{f}\right) \sigma_{m}^{2}
\end{gathered}
$$

Rearranging the terms: 


$$
\begin{aligned}
& a_{1} a_{4} e^{-3 i v \tau}+ {\left[a_{1} \mu^{2} \tau^{2} N^{2} L^{2}-2 a_{1} a_{4}-a_{1} a_{5}+a_{4}\left(1-\sigma_{f}\right) \sigma_{m}^{2}\right] e^{-2 i v \tau} } \\
&+2 a_{1} a_{5} e^{-i v \tau}+a_{1} a_{4} e^{-i v \tau} \\
&-a_{1} \mu^{2} \tau^{2} N^{2} L^{2} e^{-i v \tau}-a_{5}\left(1-\sigma_{f}\right) \sigma_{m}^{2} e^{-i v \tau}-a_{4}\left(1-\sigma_{f}\right) \sigma_{m}^{2} e^{-i v \tau} \\
&+\left(1-\sigma_{f}\right) \sigma_{m}^{2} \mu^{2} \tau^{2} N^{2} L^{2} e^{-i v \tau}+\mu^{2} \tau^{2} f^{2} a_{2} \sigma_{m}^{2} e^{-i v \tau}=\mu^{2} \tau^{2} f^{2} a_{3} \sigma_{m}^{2}-a_{5}\left(1-\sigma_{f}\right) \sigma_{m}^{2}+a_{1} a_{5}
\end{aligned}
$$

Rearranging the terms:

$$
\begin{aligned}
& a_{1} a_{4} e^{-3 i v \tau}+\left[a_{1} \mu^{2} \tau^{2} N^{2} L^{2}-2 a_{1} a_{4}-a_{1} a_{5}+a_{4}\left(1-\sigma_{f}\right) \sigma_{m}^{2}\right] e^{-2 i v \tau} \\
& +2 a_{1} a_{5} e^{-i v \tau}+a_{1} a_{4} e^{-i v \tau} \\
& -a_{1} \mu^{2} \tau^{2} N^{2} L^{2} e^{-i v \tau}-\left(a_{4}+a_{5}\right)\left(1-\sigma_{f}\right) \sigma_{m}^{2} e^{-i v \tau} \\
& \quad+\left(1-\sigma_{f}\right) \sigma_{m}^{2} \mu^{2} \tau^{2} N^{2} L^{2} e^{-i v \tau}+\mu^{2} \tau^{2} f^{2} a_{2} \sigma_{m}^{2} e^{-i v \tau}=\mu^{2} \tau^{2} f^{2} a_{3} \sigma_{m}^{2}-a_{5}\left(1-\sigma_{f}\right) \sigma_{m}^{2}+a_{1} a_{5}
\end{aligned}
$$

Rearranging the terms:

$$
\begin{aligned}
& a_{1} a_{4} e^{-3 i v \tau}+\left\{\left(\mu^{2} \tau^{2} N^{2} L^{2}+a_{5}\right) a_{1}+\left[\left(1-\sigma_{f}\right) \sigma_{m}^{2}-2 a_{1}\right] a_{4}\right\} e^{-2 i v \tau} \\
& +\left\{\mu^{2} \tau^{2}\left[\left(1-\sigma_{f}\right) \sigma_{m}^{2}-a_{1}\right] N^{2} L^{2}+f^{2} \mu^{2} \tau^{2} a_{2} \sigma_{m}^{2}-\left(a_{4}+a_{5}\right)\left(1-\sigma_{f}\right) \sigma_{m}^{2}+\left(a_{4}-2 a_{5}\right) a_{1}\right\} e^{-i v \tau} \\
& =\mu^{2} \tau^{2} f^{2} a_{3} \sigma_{m}^{2}-\left[\left(1-\sigma_{f}\right) \sigma_{m}^{2}+a_{1}\right] a_{5}
\end{aligned}
$$

Then:

$$
\begin{aligned}
& \mathcal{A} e^{-3 i v \tau}+\mathcal{B} e^{-2 i v \tau}+\mathcal{C} e^{-i v \tau}+\mathcal{D}=0 \\
& \mathcal{A} \equiv a_{1} a_{4} \\
& \mathcal{B} \equiv\left(\mu^{2} \tau^{2} N^{2} L^{2}+a_{5}\right) a_{1}+\left[\left(1-\sigma_{f}\right) \sigma_{m}^{2}-2 a_{1}\right] a_{4} \\
& \mathcal{C} \equiv \mu^{2} \tau^{2}\left[\left(1-\sigma_{f}\right) \sigma_{m}^{2}-a_{1}\right] N^{2} L^{2}+f^{2} \mu^{2} \tau^{2} a_{2} \sigma_{m}^{2}-\left(a_{4}+a_{5}\right)\left(1-\sigma_{f}\right) \sigma_{m}^{2}+\left(a_{4}-2 a_{5}\right) a_{1} \\
& \mathcal{D} \equiv-\mu^{2} \tau^{2} f^{2} a_{3} \sigma_{m}^{2}+\left[\left(1-\sigma_{f}\right) \sigma_{m}^{2}+a_{1}\right] a_{5} \\
& a_{1} \equiv \sigma_{N}^{(+4)} L^{2}+\sigma_{m}^{2} ; \quad a_{2} \equiv \sigma_{N} L^{2}+\sigma_{m}^{2} ; \quad a_{3} \equiv L^{2}+\sigma_{m}^{2} ; \quad a_{4} \equiv \mu^{2} \sigma_{N}^{(+1)} L^{2}+\sigma_{m}^{2} ; \quad a_{5} \equiv \mu^{2} L^{2}+\sigma_{f} \sigma_{m}^{2}
\end{aligned}
$$

Then: 


$$
\begin{aligned}
& \mathcal{A} e^{3 v_{i} \tau} \cos \left(3 v_{r} \tau\right)+\mathcal{B} e^{2 v_{i} \tau} \cos \left(2 v_{r} \tau\right)+\mathcal{C} e^{v_{i} \tau} \cos \left(v_{r} \tau\right)+\mathcal{D}=0 \\
& \mathcal{A} \sin \left(3 v_{r} \tau\right) e^{2 v_{i} \tau}-\mathcal{B} \sin \left(2 v_{r} \tau\right) e^{v_{i} \tau}+\mathcal{C} \sin \left(v_{r} \tau\right)=0 \\
& e^{v_{i} \tau}=\frac{-\mathcal{B} \sin \left(2 v_{r} \tau\right) \mp \sqrt{\mathcal{B}^{2} \sin ^{2}\left(2 v_{r} \tau\right)-4 \mathcal{A C} \sin \left(3 v_{r} \tau\right) \sin \left(v_{r} \tau\right)}}{2 \mathcal{A} \sin \left(3 v_{r} \tau\right)} \\
& e^{v_{i} \tau}=\frac{-\mathcal{B} \sin \left(2 v_{r} \tau\right)-\sqrt{\mathcal{B}^{2} \sin ^{2}\left(2 v_{r} \tau\right)-4 \mathcal{A C} \sin \left(3 v_{r} \tau\right) \sin \left(v_{r} \tau\right)}}{2 \mathcal{A} \sin \left(3 v_{r} \tau\right)} \quad \text { (solution) } \\
& e^{v_{i} \tau}=\frac{-\mathcal{B} \sin \left(2 v_{r} \tau\right)+\sqrt{\mathcal{B}^{2} \sin ^{2}\left(2 v_{r} \tau\right)-4 \mathcal{A C} \sin \left(3 v_{r} \tau\right) \sin \left(v_{r} \tau\right)}}{2 \mathcal{A} \sin \left(3 v_{r} \tau\right)} \quad \text { (no solution) }
\end{aligned}
$$

\section{Balance solution:}

Eqs. (181), (182) and (183):

$$
\begin{gathered}
-f\left(\underset{\sim}{i} m+\frac{1}{2 H}\right) \hat{\omega}_{z}^{(\mathrm{n})}=L^{2} \hat{B}^{(\mathrm{n})} \\
0=-f\left({\underset{\sim}{i}} m+\frac{1}{2 H}\right) \hat{\omega}_{z}^{(\mathrm{n})}-L^{2} \hat{B}^{(\mathrm{n})} \\
0=0
\end{gathered}
$$

Eqs. (176) and (177) with balance approximation:

$$
0=f \hat{\omega}_{z}^{(\mathrm{n})}+L^{2} \hat{P}_{D} \text { and } 0=\left(\underset{\sim}{i m}+\frac{1}{2 H}\right) \hat{P}_{D}+\hat{B}^{(\mathrm{n}+1)}
$$

From (209):

$$
0=-f\left(\underset{\sim}{i m}+\frac{1}{2 H}\right) \hat{\omega}_{z}^{(\mathrm{n})}+L^{2} \hat{B}^{(\mathrm{n}+1)}
$$

There is a balance within one time step difference. 
(a)

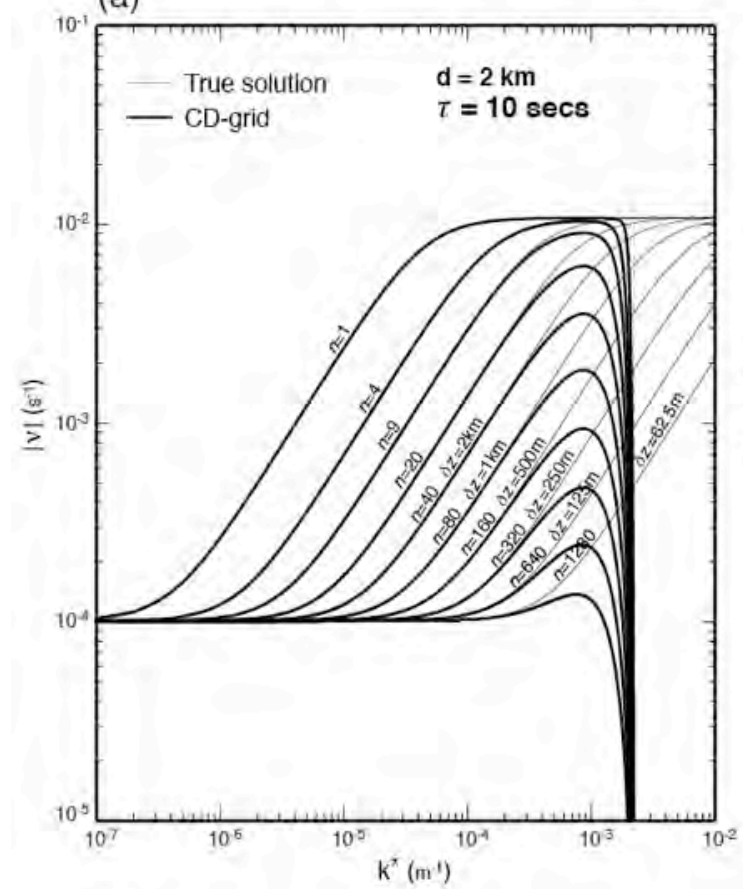

(c)

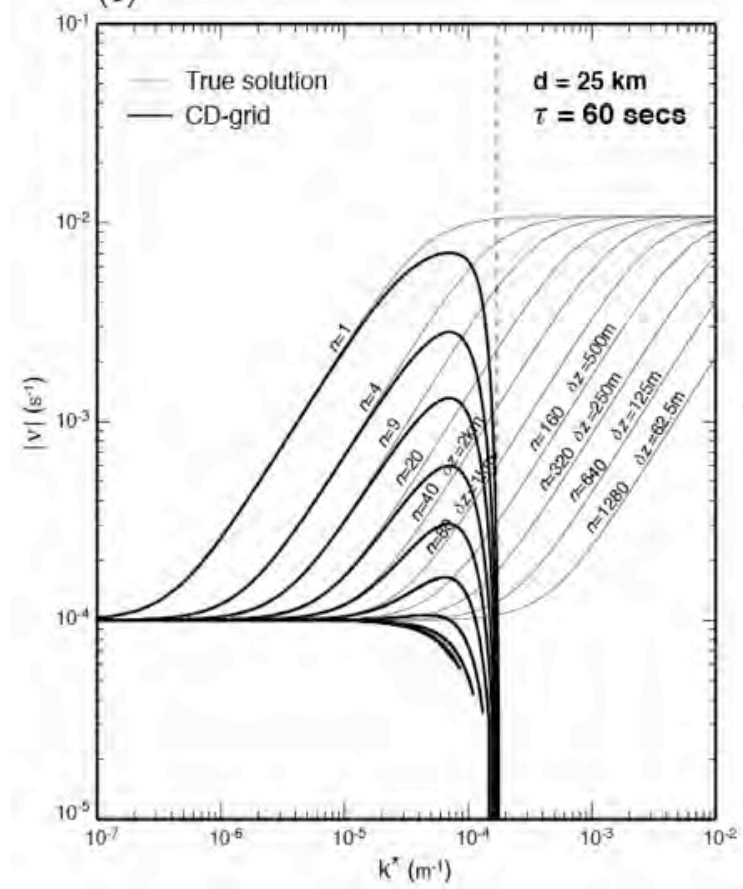

(b)

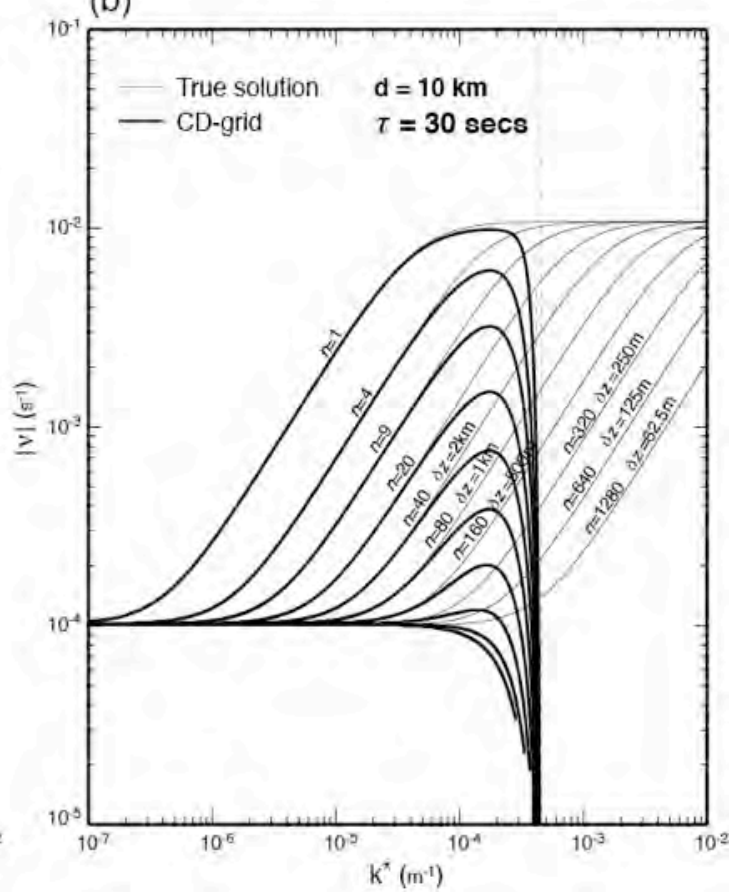

(d)

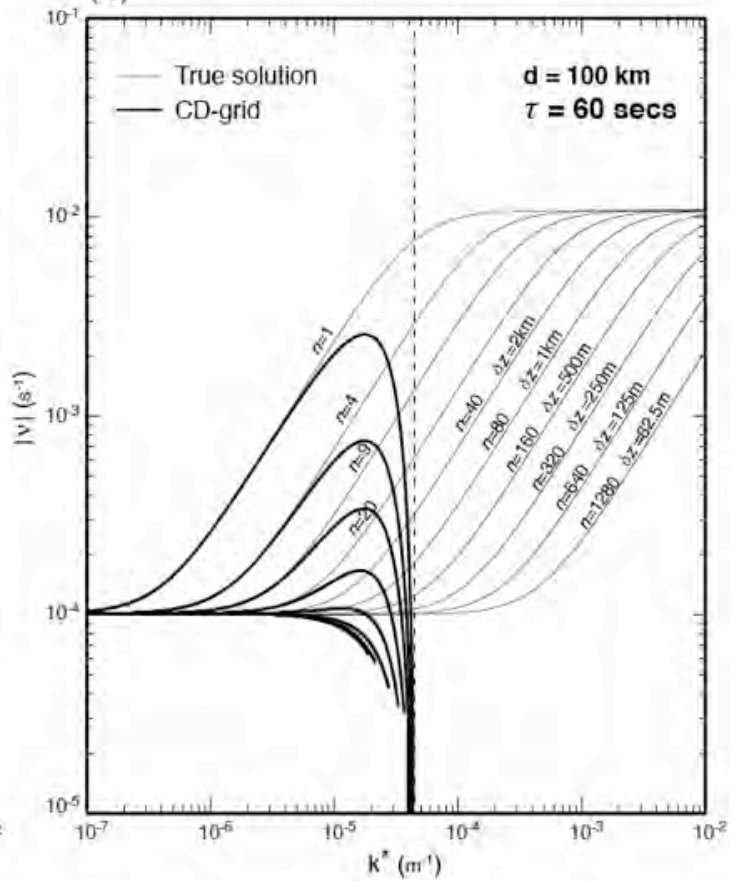

Figure Scheme IV. (runs_CD_grid_time_discrete_scheme_7) 


\section{Scheme V:}

$$
\begin{aligned}
& \hat{\omega}_{z}^{(*)}=\mu \hat{\omega}_{z}^{(\mathrm{n})}-\frac{\tau}{2} f \hat{D}^{(\mathrm{n})} \\
& \hat{D}^{(*)}=\mu \hat{D}^{(\mathrm{n})}+\frac{\tau}{2}\left(f \mu \hat{\omega}_{z}^{(*)}+L^{2} \hat{P}_{C}\right) \\
& \hat{w}^{(*)}=\hat{w}^{(\mathrm{n})}+\frac{\tau}{2}\left[-\left(\underset{\sim}{i m}+\frac{1}{2 H}\right) \hat{P}_{C}+\hat{B}^{(\mathrm{n})}\right] \\
& \hat{B}^{(*)}=\hat{B}^{(\mathrm{n})}-\frac{\tau}{2} N^{2} \hat{w}^{(\mathrm{n})} \\
& \hat{D}^{(*)}+\left(\underset{\sim}{i} m-\frac{1}{2 H}\right) \hat{w}^{(*)}=0
\end{aligned}
$$

$$
\begin{aligned}
& \hat{\omega}_{z}^{(n+1)}=\hat{\omega}_{z}^{(\mathrm{n})}-\tau f \hat{D}^{(*)}(305) \\
& \hat{D}^{(\mathrm{n}+1)}=\hat{D}^{(\mathrm{n})}+\tau\left(f \mu \hat{\omega}_{z}^{(\mathrm{n}+1)}+\mu L^{2} \hat{P}_{D}\right)(306) \\
& \hat{w}^{(\mathrm{n}+1)}=\hat{w}^{(\mathrm{n})}+\tau\left[-\left(\underset{\sim}{i m}+\frac{1}{2 H}\right) \hat{P}_{D}+\hat{B}^{(n+1)}\right](307) \\
& \hat{B}^{(\mathrm{n}+1)}=\hat{B}^{(\mathrm{n})}-\tau N^{2} \hat{w}^{(*)}(308) \\
& \mu \hat{D}^{(\mathrm{n}+1)}+\left(\underset{\sim}{i m}-\frac{1}{2 H}\right) \hat{w}^{(\mathrm{n}+1)}=0(309)
\end{aligned}
$$

\section{Predictor-step equations:}

Eliminating $\hat{P}_{C}$ between (301) and (302) using (300), (304) and (309):

$$
\begin{array}{r}
\left(L^{2}+m^{2}+\frac{1}{4 H^{2}}\right) \hat{D}^{(*)}=\mu\left[L^{2}+\left(m^{2}+\frac{1}{4 H^{2}}\right)\left(1-\frac{\tau^{2} f^{2}}{4}\right)\right] \hat{D}^{(\mathrm{n})}+\frac{\tau}{2} f \mu^{2}\left(m^{2}+\frac{1}{4 H^{2}}\right) \hat{\omega}_{z}^{(\mathrm{n})} \\
-\frac{\tau}{2}\left(\underset{\sim}{\left.\underset{\sim}{i} m-\frac{1}{2 H}\right) L^{2} \hat{B}^{(\mathrm{n})}}\right.
\end{array}
$$

Using (304) in (3.10):

$$
\begin{aligned}
-\left(\underset{\sim}{i m}-\frac{1}{2 H}\right)\left(L^{2}+m^{2}+\frac{1}{4 H^{2}}\right) \hat{w}^{(*)}= & \mu\left[L^{2}+\left(m^{2}+\frac{1}{4 H^{2}}\right)\left(1-\frac{\tau^{2} f^{2}}{4}\right)\right] \hat{D}^{(\mathrm{n})} \\
& +\frac{\tau}{2} f \mu^{2}\left(m^{2}+\frac{1}{4 H^{2}}\right) \hat{\omega}_{z}^{(\mathrm{n})}-\frac{\tau}{2}\left(\underset{\sim}{i m}-\frac{1}{2 H}\right) L^{2} \hat{B}^{(\mathrm{n})}
\end{aligned}
$$

\section{Corrector-step equations:}

Using (310) in (305):

$$
\begin{aligned}
\left(L^{2}+m^{2}+\frac{1}{4 H^{2}}\right)\left(\hat{\boldsymbol{\omega}}_{z}^{(\mathrm{n}+1)}-\hat{\boldsymbol{\omega}}_{z}^{(\mathrm{n})}\right) & =-\mu^{2} \frac{\tau^{2} f^{2}}{2}\left(m^{2}+\frac{1}{4 H^{2}}\right) \hat{\boldsymbol{\omega}}_{z}^{(\mathrm{n})} \\
& -\tau f \mu\left[L^{2}+\left(m^{2}+\frac{1}{4 H^{2}}\right)\left(1-\frac{\tau^{2} f^{2}}{4}\right)\right] \hat{D}^{(\mathrm{n})}+\tau f \frac{\tau}{2}\left(\underset{\sim}{i m} m-\frac{1}{2 H}\right) L^{2} \hat{B}^{(\mathrm{n})}
\end{aligned}
$$

Eliminating $\hat{P}_{D}$ between (306) and (307) using (305), (308) and (309): 


$$
\left(\mu^{2} L^{2}+m^{2}+\frac{1}{4 H^{2}}\right)\left(\hat{D}^{(n+1)}-\hat{D}^{(\mathrm{n})}\right)=\tau f \mu\left(m^{2}+\frac{1}{4 H^{2}}\right) \hat{\omega}_{z}^{(\mathrm{n}+1)}-\tau \mu L^{2}\left(\underset{\sim}{i m}-\frac{1}{2 H}\right) \hat{B}^{(n+1)}
$$

Using (311) in (308):

$$
\begin{aligned}
& \left(L^{2}+m^{2}+\frac{1}{4 H^{2}}\right)\left(\underset{\sim}{i m}-\frac{1}{2 H}\right)\left(\hat{B}^{(\mathrm{n}+1)}-\hat{B}^{(\mathrm{n})}\right)+\frac{\tau^{2} N^{2}}{2} L^{2}\left(\underset{\sim}{i m}-\frac{1}{2 H}\right) \hat{B}^{(\mathrm{n})}= \\
& \tau N^{2} \mu\left[L^{2}+\left(m^{2}+\frac{1}{4 H^{2}}\right)\left(1-\frac{\tau^{2} f^{2}}{4}\right)\right] \hat{D}^{(\mathrm{n})}+f \mu^{2} \frac{\tau^{2} N^{2}}{2}\left(m^{2}+\frac{1}{4 H^{2}}\right) \hat{\omega}_{z}^{(\mathrm{n})}
\end{aligned}
$$

Eqs. (312)-(314) are the equations of the system.

Defining $\sigma_{f}^{(-4)} \equiv 1-\frac{\tau^{2} f^{2}}{4}$ and using $\hat{\Phi}^{(n+1)}=e^{-i v \tau} \hat{\Phi}^{(\mathrm{n})}$ in (312)-(314):

$$
\begin{gathered}
\left(L^{2}+\sigma_{m}^{2}\right)\left(e^{-i v \tau}-1\right) \hat{\omega}_{z}=-\mu^{2}\left(1-\sigma_{f}\right) \sigma_{m}^{2} \hat{\omega}_{z}-\tau f \mu\left(L^{2}+\sigma_{f}^{(-4)} \sigma_{m}^{2}\right) \hat{D}+\tau f \frac{\tau}{2}\left(\underset{\sim}{i} m-\frac{1}{2 H}\right) L^{2} \hat{B} \\
\left(\mu^{2} L^{2}+\sigma_{m}^{2}\right)\left(e^{-i v \tau}-1\right) \hat{D}=\tau f \mu \sigma_{m}^{2} e^{-i v \tau} \hat{\omega}_{z}-\tau \mu e^{-i v \tau}\left(\underset{\sim}{i m}-\frac{1}{2 H}\right) L^{2} \hat{B} \\
\left(L^{2}+\sigma_{m}^{2}\right)\left(e^{-i v \tau}-1\right)\left(\underset{\sim}{i} m-\frac{1}{2 H}\right) \hat{B}+\left(1-\sigma_{N}\right)\left(\underset{\sim}{i} m-\frac{1}{2 H}\right) L^{2} \hat{B}= \\
\tau N^{2} \mu\left(L^{2}+\sigma_{f}^{(-4)} \sigma_{m}^{2}\right) \hat{D}+f \mu^{2}\left(1-\sigma_{N}\right) \sigma_{m}^{2} \hat{\omega}_{z}
\end{gathered}
$$

Eliminating D between (315) and (317):

$$
N^{2} \hat{\omega}_{z}+f\left(\underset{\sim}{\operatorname{im}}-\frac{1}{2 H}\right) \hat{B}=0
$$

Using (318) in (316):

$$
f\left(\mu^{2} L^{2}+\sigma_{m}^{2}\right)\left(e^{-i v \tau}-1\right) \hat{D}=\tau \mu\left(N^{2} L^{2}+f^{2} \sigma_{m}^{2}\right) e^{-i v \tau} \hat{\omega}_{z}
$$

Using (318) in (317):

$$
\left[\left(L^{2}+\sigma_{m}^{2}\right)\left(e^{-i v \tau}-1\right) N^{2}+\left(1-\sigma_{N}\right)\left(L^{2} N^{2}+f^{2} \mu^{2} \sigma_{m}^{2}\right)\right] \hat{\omega}_{z}=-\tau f N^{2} \mu\left(L^{2}+\sigma_{f}^{(-4)} \sigma_{m}^{2}\right) \hat{D}
$$

Eliminating $\hat{\omega}_{z}(319)$ in (320): 


$$
\begin{array}{r}
{\left[\left(L^{2}+\sigma_{m}^{2}\right)\left(e^{-i v \tau}-1\right) N^{2}+\left(1-\sigma_{N}\right)\left(L^{2} N^{2}+f^{2} \mu^{2} \sigma_{m}^{2}\right)\right]\left(\mu^{2} L^{2}+\sigma_{m}^{2}\right)\left(e^{-i v \tau}-1\right) \hat{D}=} \\
-\tau^{2} \mu^{2} N^{2}\left(N^{2} L^{2}+f^{2} \sigma_{m}^{2}\right)\left(L^{2}+\sigma_{f}^{(-4)} \sigma_{m}^{2}\right) e^{-i v \tau} \hat{D}
\end{array}
$$

Nontrivial solutions:

$$
\begin{array}{r}
\left(L^{2}+\sigma_{m}^{2}\right)\left(\mu^{2} L^{2}+\sigma_{m}^{2}\right)\left(e^{-i v \tau}-1\right)^{2}+\frac{\tau^{2}}{2} \\
\left(\mu^{2} L^{2}+\sigma_{m}^{2}\right)\left(N^{2} L^{2}+f^{2} \mu^{2} \sigma_{m}^{2}\right)\left(e^{-i v \tau}-1\right)= \\
-\tau^{2} \mu^{2}\left(N^{2} L^{2}+f^{2} \sigma_{m}^{2}\right)\left(L^{2}+\sigma_{f}^{(-4)} \sigma_{m}^{2}\right) e^{-i v \tau}
\end{array}
$$

Arranging the terms:

$$
\begin{aligned}
\left(L^{2}+\sigma_{m}^{2}\right)\left(\mu^{2} L^{2}+\sigma_{m}^{2}\right)\left(e^{-2 i v \tau}-2 e^{-i v \tau}+1\right) & \\
+\frac{\tau^{2}}{2}\left(\mu^{2} L^{2}+\sigma_{m}^{2}\right)\left(N^{2} L^{2}+f^{2} \mu^{2} \sigma_{m}^{2}\right) e^{-i v \tau}+\tau^{2} \mu^{2}\left(N^{2} L^{2}+f^{2} \sigma_{m}^{2}\right)\left(L^{2}+\sigma_{f}^{(-4)} \sigma_{m}^{2}\right) e^{-i v \tau} & -\frac{\tau^{2}}{2}\left(\mu^{2} L^{2}+\sigma_{m}^{2}\right)\left(N^{2} L^{2}+f^{2} \mu^{2} \sigma_{m}^{2}\right)=0
\end{aligned}
$$

Rearranging the terms:

$$
\begin{aligned}
\left(L^{2}+\sigma_{m}^{2}\right)\left(\mu^{2} L^{2}+\sigma_{m}^{2}\right) e^{-2 i v \tau} & \\
+\left\{-\left(\mu^{2} L^{2}+\sigma_{m}^{2}\right)\left[2\left(L^{2}+\sigma_{m}^{2}\right)-\frac{\tau^{2}}{2}(\right.\right. & \left.\left.\left.N^{2} L^{2}+f^{2} \mu^{2} \sigma_{m}^{2}\right)\right]+\tau^{2} \mu^{2}\left(N^{2} L^{2}+f^{2} \sigma_{m}^{2}\right)\left(L^{2}+\sigma_{f}^{(-4)} \sigma_{m}^{2}\right)\right\} e^{-i v \tau} \\
& +\left(\mu^{2} L^{2}+\sigma_{m}^{2}\right)\left[\left(L^{2}+\sigma_{m}^{2}\right)-\frac{\tau^{2}}{2}\left(N^{2} L^{2}+f^{2} \mu^{2} \sigma_{m}^{2}\right)\right]=0
\end{aligned}
$$

Using $\sigma_{f}^{(\mu)} \equiv 1-\mu^{2} \frac{\tau^{2} f^{2}}{2}$ in (324) and arranging the terms:

$$
\begin{aligned}
\left(L^{2}+\sigma_{m}^{2}\right)\left(\mu^{2} L^{2}+\sigma_{m}^{2}\right) e^{-2 i v \tau} & \\
+\left[\tau^{2} \mu^{2}\left(N^{2} L^{2}+f^{2} \sigma_{m}^{2}\right)\left(L^{2}+\sigma_{f}^{(-4)} \sigma_{m}^{2}\right)\right. & \left.-\left(\mu^{2} L^{2}+\sigma_{m}^{2}\right)\left(L^{2}+\sigma_{m}^{2}+\sigma_{N} L^{2}+\sigma_{f}^{(\mu)} \sigma_{m}^{2}\right)\right] e^{-i v \tau} \\
& +\left(\mu^{2} L^{2}+\sigma_{m}^{2}\right)\left(\sigma_{N} L^{2}+\sigma_{f}^{(\mu)} \sigma_{m}^{2}\right)=0
\end{aligned}
$$

Then 


$$
\begin{array}{r}
\left(L^{2}+\sigma_{m}^{2}\right) e^{-2 i v \tau}+\left[\tau^{2} \mu^{2}\left(\frac{N^{2} L^{2}+f^{2} \sigma_{m}^{2}}{\mu^{2} L^{2}+\sigma_{m}^{2}}\right)\left(L^{2}+\sigma_{f}^{(-4)} \sigma_{m}^{2}\right)-\left(L^{2}+\sigma_{m}^{2}+\sigma_{N} L^{2}+\sigma_{f}^{(\mu)} \sigma_{m}^{2}\right)\right] e^{-i v \tau} \\
+\left(\sigma_{N} L^{2}+\sigma_{f}^{(\mu)} \sigma_{m}^{2}\right)=0
\end{array}
$$

Then:

$$
\begin{aligned}
\left(L^{2}+\sigma_{m}^{2}\right) e^{2 v_{i} \tau} \cos \left(2 \nu_{r} \tau\right) & \\
+ & {\left[\tau^{2} \mu^{2}\left(\frac{N^{2} L^{2}+f^{2} \sigma_{m}^{2}}{\mu^{2} L^{2}+\sigma_{m}^{2}}\right)\left(L^{2}+\sigma_{f}^{(-4)} \sigma_{m}^{2}\right)-\left(L^{2}+\sigma_{m}^{2}+\sigma_{N} L^{2}+\sigma_{f}^{(\mu)} \sigma_{m}^{2}\right)\right] e^{v_{i} \tau} \cos \left(v_{r} \tau\right) } \\
+\left(\sigma_{N} L^{2}+\sigma_{f}^{(\mu)} \sigma_{m}^{2}\right) & =0
\end{aligned}
$$

and

$$
e^{v_{i} \tau}=-\left[\tau^{2} \mu^{2}\left(\frac{N^{2} L^{2}+f^{2} \sigma_{m}^{2}}{\mu^{2} L^{2}+\sigma_{m}^{2}}\right)\left(\frac{L^{2}+\sigma_{f}^{(-4)} \sigma_{m}^{2}}{L^{2}+\sigma_{m}^{2}}\right)-\left(1+\frac{\sigma_{N} L^{2}+\sigma_{f}^{(\mu)} \sigma_{m}^{2}}{L^{2}+\sigma_{m}^{2}}\right)\right] \frac{\sin \left(v_{r} \tau\right)}{\sin \left(2 \nu_{r} \tau\right)}
$$

\section{Balance solution:}

Eqs. (312)-(314) with balance assumption:

$$
\begin{gathered}
0=\mu^{2} f\left(\underset{\sim}{i} m+\frac{1}{2 H}\right) \hat{\omega}_{z}^{(\mathrm{n})}+L^{2} \hat{B}^{(\mathrm{n})} \\
0=-f\left({\underset{\sim}{i}}_{\sim} m+\frac{1}{2 H}\right) \hat{\omega}_{z}^{(\mathrm{n}+1)}-L^{2} \hat{B}^{(n+1)} \\
L^{2} \hat{B}^{(\mathrm{n})}=-\mu^{2} f\left(\sim_{\sim}^{i} m+\frac{1}{2 H}\right) \hat{\omega}_{z}^{(\mathrm{n})}
\end{gathered}
$$

There is no unique balance solution. 
(a)

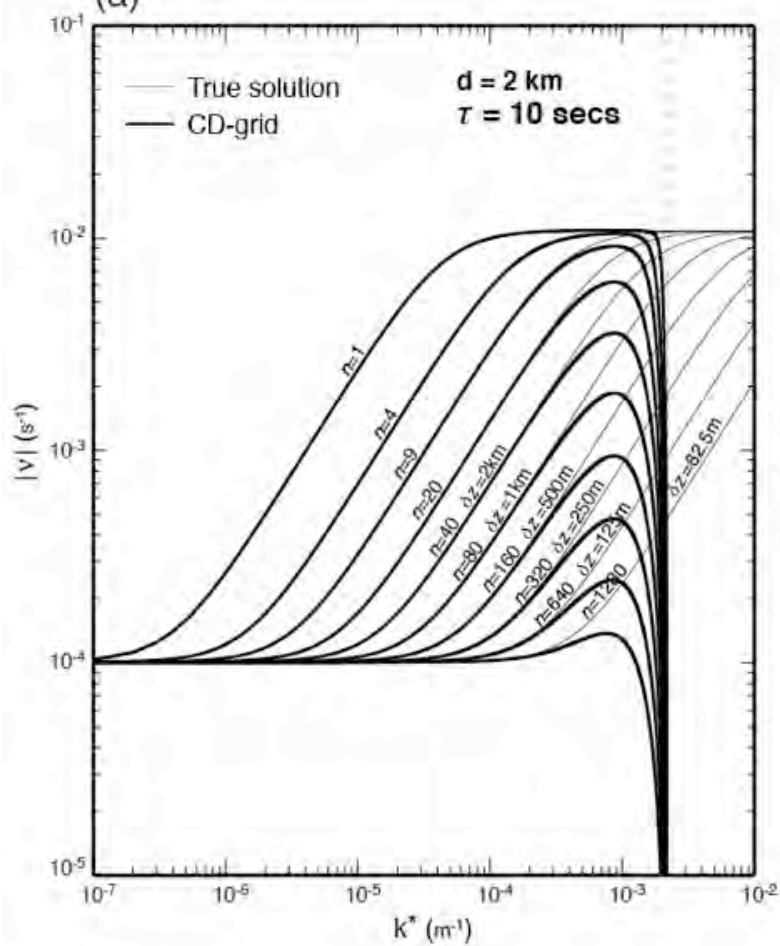

(c)

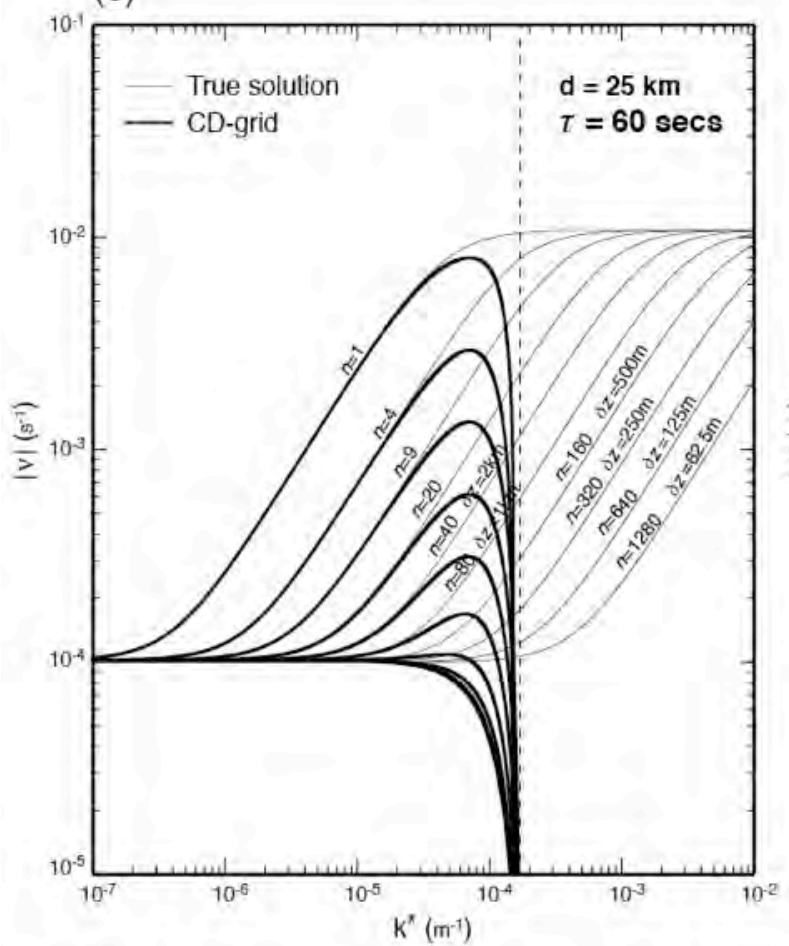

(b)

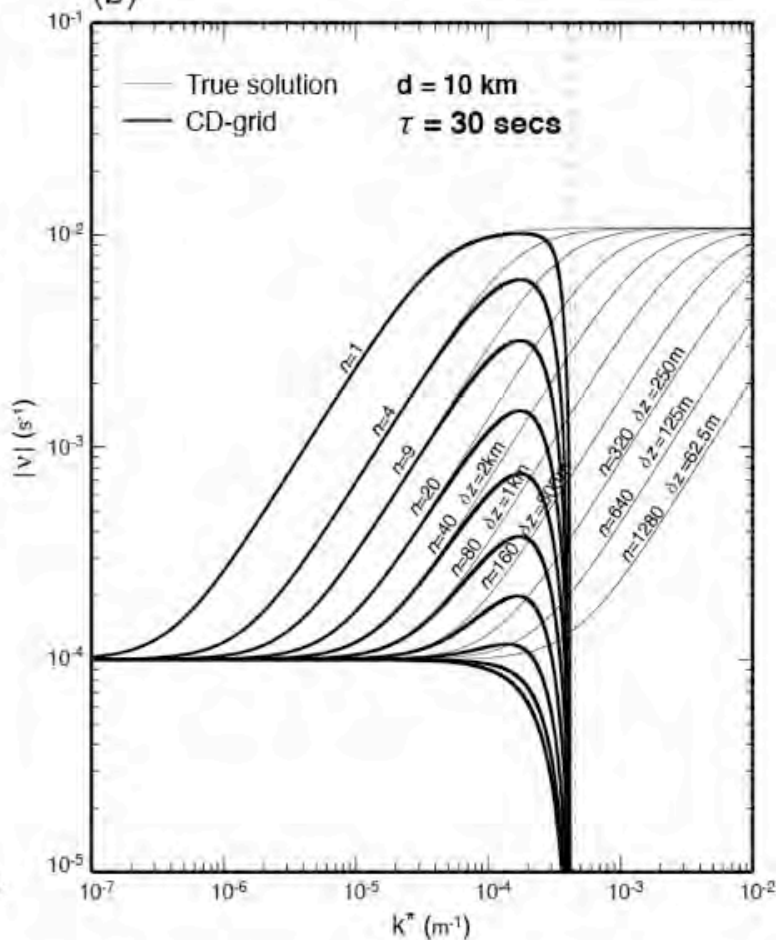

(d)

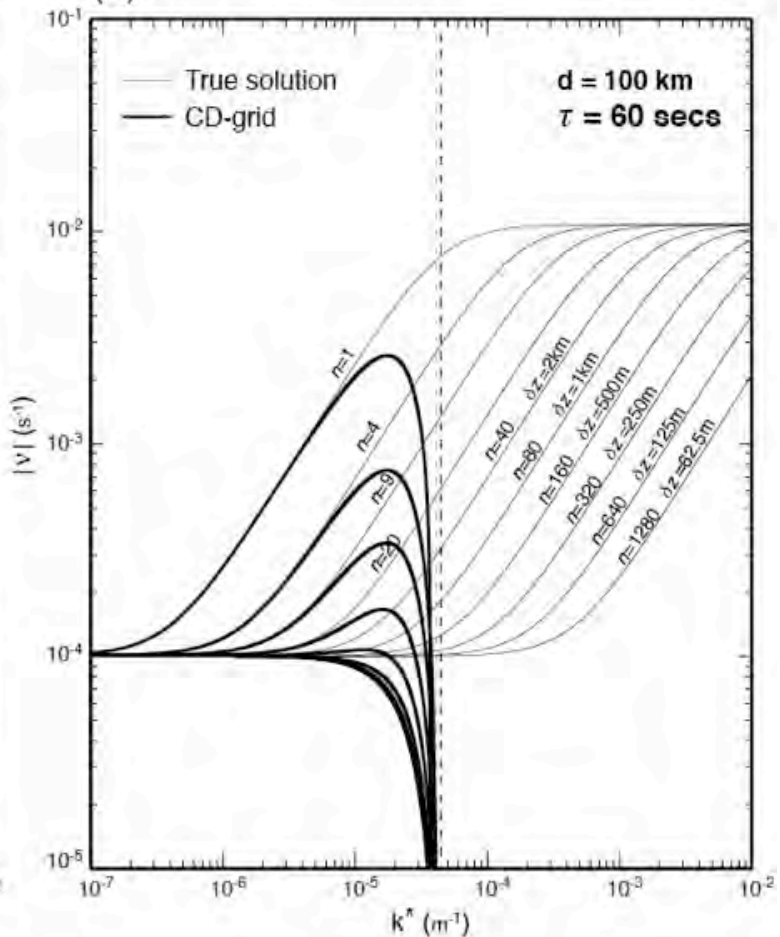

Figure. Scheme V: (runs_CD_grid_time_discrete_scheme_5) 


\section{Rossby modes}

\section{Scheme R1:}

Baroclinic modes:

C-grid equations:

Assumptions $v=\frac{1}{f_{0}} \frac{\partial P}{\partial x}, \frac{\partial D}{\partial t}=0$ and $\frac{\partial w}{\partial t}=0$.

Thus, $D$ is a diagnostic variable. It is assumed that $D$ is defined on the CD-grid's corners.

$\mathrm{P}$ is defined at the centers. Thus, it is averaged to corners.

$$
\frac{\partial}{\partial t}\left(\omega_{z}\right)_{i+1 / 2, j+1 / 2}=-f_{0} D_{i+1 / 2, j+1 / 2}-\frac{\beta}{f_{0}} \frac{\bar{P}_{i+3 / 2, j+1 / 2}-\bar{P}_{i-1 / 2, j+1 / 2}}{2 d}
$$

where

$$
\bar{P}_{i+1 / 2, j+1 / 2} \equiv \frac{1}{4}\left(P_{i+1, j+1}+P_{i+1, j-1}+P_{i-1, j+1}+P_{i-1, j-1}\right)
$$

Divergence equation from (M.8):

$$
0=-\frac{P_{i+1, j}+P_{i-1, j}+P_{i, j+1}+P_{i, j-1}-4 P_{i, j}}{d^{2}}+f \frac{1}{4}\left[\left(\omega_{z}\right)_{i+1 / 2, j+1 / 2}+\left(\omega_{z}\right)_{i-1 / 2, j+1 / 2}+\left(\omega_{z}\right)_{i+1 / 2, j-1 / 2}+\left(\omega_{z}\right)_{i-1 / 2, j-1 / 2}\right]
$$

Vertical momentum equation:

$$
0=-\left[\frac{\partial}{\partial z}-\left(\frac{1}{2 \rho_{0}} \frac{\partial \rho_{0}}{\partial z}\right)\right] P_{i, j}+B_{i, j}
$$

Thermodynamic equation: 


$$
\frac{\partial B_{i, j}}{\partial t}=-N^{2} w_{i, j}
$$

Mass continuity equation:

$$
\frac{1}{4}\left(D_{i+1 / 2, j+1 / 2}+D_{i-1 / 2, j+1 / 2}+D_{i+1 / 2, j-1 / 2}+D_{i-1 / 2, j-1 / 2}\right)+\left[\frac{\partial}{\partial z}+\left(\frac{1}{2 \rho_{0}} \frac{\partial \rho_{0}}{\partial z}\right)\right] w_{i, j}=0
$$

\section{D-grid equations:}

Assumptions $v=\frac{1}{f_{0}} \frac{\partial P}{\partial x}, \frac{\partial D}{\partial t}=0$ and $\frac{\partial w}{\partial t}=0$.

Thus, $D$ is a diagnostic variable. It is assumed that $D$ is defined on the CD-grid's corners.

$$
\frac{\partial}{\partial t}\left(\omega_{z}\right)_{i, j}=-f_{0} \frac{1}{4}\left(D_{i+1 / 2, j+1 / 2}+D_{i-1 / 2, j+1 / 2}+D_{i+1 / 2, j-1 / 2}+D_{i-1 / 2, j-1 / 2}\right)-\frac{\beta}{f_{0}} \frac{P_{i+1, j}-P_{i-1, j}}{2 d}
$$

Divergence equation from (M.8):

$$
0=-\frac{P_{i+1, j}+P_{i-1, j}+P_{i, j+1}+P_{i, j-1}-4 P_{i, j}}{d^{2}}+f_{0}\left(\omega_{z}\right)_{i, j}
$$

Vertical momentum equation:

$$
0=-\left[\frac{\partial}{\partial z}-\left(\frac{1}{2 \rho_{0}} \frac{\partial \rho_{0}}{\partial z}\right)\right] P_{i, j}+B_{i, j}
$$

Thermodynamic equation:

$$
\frac{\partial B_{i, j}}{\partial t}=-N^{2} w_{i, j}
$$


Mass continuity equation:

$$
\frac{1}{4}\left(D_{i+1 / 2, j+1 / 2}+D_{i-1 / 2, j+1 / 2}+D_{i+1 / 2, j-1 / 2}+D_{i-1 / 2, j-1 / 2}\right)+\left[\frac{\partial}{\partial z}+\left(\frac{1}{2 \rho_{0}} \frac{\partial \rho_{0}}{\partial z}\right)\right] w_{i, j}=0
$$

\section{C-grid equations:}

$$
\begin{gathered}
\frac{\partial}{\partial t} \hat{\omega}_{z}=-f \hat{D}-i \mu \frac{\beta}{f_{0}} \tilde{\xi}_{k} \hat{P} \\
0=\left(\xi^{2} k^{2}+\eta^{2} \ell^{2}\right) \hat{P}+\mu f_{0} \hat{\omega}_{z} \\
0=-\left[\frac{\partial}{\partial z}-\left(\frac{1}{2 \rho_{0}} \frac{\partial \rho_{0}}{\partial z}\right)\right] \hat{P}+\hat{B} \\
\frac{\partial}{\partial t} \hat{B}=-N^{2} \hat{w} \\
\mu \hat{D}+\left[\frac{\partial}{\partial z}+\left(\frac{1}{2 \rho_{0}} \frac{\partial \rho_{0}}{\partial z}\right)\right] \hat{w}=0
\end{gathered}
$$

\section{D-grid equations:}

$$
\frac{\partial}{\partial t} \hat{\omega}_{z}=-\mu f_{0} \hat{D}-\frac{\beta}{f_{0}} \tilde{\xi}_{k} \hat{P}
$$

$D$ is defined at the corners, thus averaged in this equation

$$
\begin{gathered}
0=\left(\xi^{2} k^{2}+\eta^{2} \ell^{2}\right) \hat{P}+f_{0} \hat{\omega}_{z} \\
0=-\left[\frac{\partial}{\partial z}-\left(\frac{1}{2 \rho_{0}} \frac{\partial \rho_{0}}{\partial z}\right)\right] \hat{P}+\hat{B}
\end{gathered}
$$




$$
\begin{gathered}
\frac{\partial}{\partial t} \hat{B}=-N^{2} \hat{w} \\
\mu \hat{D}+\left[\frac{\partial}{\partial z}+\left(\frac{1}{2 \rho_{0}} \frac{\partial \rho_{0}}{\partial z}\right)\right] \hat{w}=0
\end{gathered}
$$

\section{Predictor-step equations on the C-grid:}

$$
\hat{\omega}_{z}^{(*)}=\mu \hat{\omega}_{z}^{(\mathrm{n})}-\frac{1}{2} \tau f_{0} \hat{D}-\underset{\sim}{i} \frac{1}{2} \tau \mu \frac{\beta}{f_{0}} \tilde{\xi}_{k} \hat{P}
$$

$\hat{\omega}_{z}^{(*)}$ is defined at the corners of the CD grid. $\hat{\omega}_{z}^{(\mathrm{n})}$ and $\hat{P}$ are defined at the centers, thus they are averaged (multiplied by $\mu$ ).

$$
0=L^{2} \hat{P}+f_{0} \hat{\boldsymbol{\omega}}_{z}^{(\mathrm{n})}
$$

$\hat{\omega}_{z}^{(\mathrm{n})}$ is defined at the corners, thus it is not averaged.

$$
\begin{gathered}
0=-\left(\underset{\sim}{i} m+\frac{1}{2 H}\right) \hat{P}+\hat{B}^{(\mathrm{n})} \\
\hat{B}^{(*)}=\hat{B}^{(\mathrm{n})}-\frac{1}{2} \tau N^{2} \hat{w} \\
\mu \hat{D}+\left(\underset{\sim}{i} m-\frac{1}{2 H}\right) \hat{w}=0 \\
\hat{\omega}_{z}^{(\mathrm{n}+1)}=\hat{\omega}_{z}^{(\mathrm{n})}-\tau f_{0} \mu \hat{D}-\underset{\sim}{i} \tau \frac{\beta}{f_{0}} \tilde{\xi}_{k} \hat{P}
\end{gathered}
$$




\section{Corrector-step equations on the D-grid:}

$$
\hat{\omega}_{z}^{(\mathrm{n}+1)}=\hat{\omega}_{z}^{(\mathrm{n})}-\tau f_{0} \mu \hat{D}-\underset{\sim}{i} \tau \frac{\beta}{f_{0}} \tilde{\xi}_{k} \hat{P}
$$

$D$ is defined at the corners, thus it is averaged to the centers.

$$
0=f_{0} \hat{\omega}_{z}^{(*)}+\mu L^{2} \hat{P}
$$

(RD.14) is applied to the corners. $\hat{\omega}_{z}^{(*)}$ is defined at the corners. $\hat{P}$ is defined at the centers, thus it is averaged to the corners.

$$
\begin{gathered}
0=-\left(\underset{\sim}{\operatorname{im}} m+\frac{1}{2 H}\right) \hat{P}+\hat{B}^{(*)} \\
\hat{B}^{(\mathrm{n}+1)}=\hat{B}^{(\mathrm{n})}-\tau N^{2} \hat{w} \\
\mu \hat{D}+\left(\underset{\sim}{\operatorname{im}}-\frac{1}{2 H}\right) \hat{w}=0
\end{gathered}
$$

Rewriting equations in a table: 


\begin{tabular}{|ll|ll|}
\hline \multicolumn{1}{l}{ Predictor step on the C-grid: } & \multicolumn{1}{l}{ Corrector step on the D-grid: } \\
$\hat{\omega}_{z}^{(*)}=\mu \hat{\omega}_{z}^{(\mathrm{n})}-\frac{1}{2} \tau f_{0} \hat{D}-\underset{\sim}{i} \frac{1}{2} \tau \mu \frac{\beta}{f_{0}} \tilde{\xi}_{k} \hat{P}$ & $(\mathrm{RC} .13)$ & $\hat{\omega}_{z}^{(\mathrm{n}+1)}=\hat{\omega}_{z}^{(\mathrm{n})}-\tau f_{0} \mu \hat{D}-\underset{\sim}{i} \tau \frac{\beta}{f_{0}} \tilde{\xi}_{k} \hat{P}$
\end{tabular}

Using (RC.13) in (RD.14):

$$
0=\mu L^{2} \hat{P}+f_{0}\left(\mu \hat{\omega}_{z}^{(n)}-\frac{1}{2} \tau f_{0} \hat{D}^{(n)}-i \frac{1}{2} \tau \mu \frac{\beta}{f_{0}} \tilde{\xi}_{k} \hat{P}\right)
$$

Using (RC.16) in (RD.15):

$$
0=-\left(i m+\frac{1}{2 H}\right) \hat{P}+\hat{B}^{(\mathrm{n})}-\frac{1}{2} \tau N^{2} \hat{w}
$$

Using $\hat{\Phi}^{(\mathrm{n}+1)}=e^{-i \nu \tau} \hat{\Phi}^{(\mathrm{n})}$ in (RD.13) and dropping the time stamp (n):

$$
\left(e^{-i v \tau}-1\right) \hat{\omega}_{z}=-\tau f_{0} \mu \hat{D}-i \tau \frac{\beta}{f_{0}} \tilde{\xi}_{k} \hat{P}
$$

Using $\hat{\Phi}^{(\mathrm{n}+1)}=e^{-i v \tau} \hat{\Phi}^{(\mathrm{n})}$ in (Ro.1) and dropping the time stamp (n):

$$
0=\mu L^{2} \hat{P}+f_{0} \mu \hat{\omega}_{z}-\frac{1}{2} \tau f_{0}^{2} \hat{D}-i \frac{1}{2} \tau \mu \beta \tilde{\xi} k \hat{P}
$$

Using (RD.17) in (Ro.2) with $\hat{\Phi}^{(\mathrm{n}+1)}=e^{-i v \tau} \hat{\Phi}^{(\mathrm{n})}$ and dropping the time stamp (n):

$$
0=-\left(\underset{\sim}{i} m-\frac{1}{2 H}\right)\left(i m+\frac{1}{2 H}\right) \hat{P}+\left(\underset{\sim}{i} m-\frac{1}{2 H}\right) \hat{B}+\frac{1}{2} \tau N^{2} \mu \hat{D}
$$

Rewriting (Ro.5): 


$$
0=\left(m^{2}+\frac{1}{4 H^{2}}\right) \hat{P}+\left(\underset{\sim}{i} m-\frac{1}{2 H}\right) \hat{B}+\frac{1}{2} \tau N^{2} \mu \hat{D}
$$

Using $\hat{\Phi}^{(\mathrm{n}+1)}=e^{-i v \tau} \hat{\Phi}^{(\mathrm{n})}$ in (RD.16) and dropping the time stamp (n):

$$
\left(e^{-i v \tau}-1\right) \hat{B}=-\tau N^{2} \hat{w}
$$

Using (RD.17) in (Ro.7):

$$
\left(e^{-i v \tau}-1\right)\left(\underset{\sim}{i m}-\frac{1}{2 H}\right) \hat{B}=\tau N^{2} \mu \hat{D}
$$

Using (Ro.8) in (Ro.6):

$$
0=\left(e^{-i v \tau}-1\right)\left(m^{2}+\frac{1}{4 H^{2}}\right) \hat{P}+\frac{1}{2} \tau N^{2} \mu\left(e^{-i v \tau}+1\right) \hat{D}
$$

Eqs. (Ro.3), (Ro.4) and (Ro.9) are the equations of the system.

Eliminating $\hat{\omega}$ between (Ro.3) and (Ro.4)

$$
0=\left(e^{-i v \tau}-1\right) \mu L^{2} \hat{P}-i \frac{1}{2} \tau \mu \beta \tilde{\xi} k\left(e^{-i v \tau}+1\right) \hat{P}-\tau f_{0}^{2}\left[\mu^{2}+\frac{1}{2}\left(e^{-i v \tau}-1\right)\right] \hat{D}
$$

Eliminating $\hat{D}$ between (Ro.9) and (Ro.10):

$$
\begin{aligned}
0=N^{2} \mu\left(e^{-2 i v \tau}-1\right) \mu L^{2} \hat{P}-i \frac{1}{2} N^{2} \mu\left(e^{-i v \tau}+1\right)^{2} \tau \mu \beta \tilde{\xi}_{k} \hat{P} \\
+f_{0}^{2}\left[2 \mu^{2}\left(e^{-i v \tau}-1\right)+\left(e^{-i v \tau}-1\right)^{2}\right]\left(m^{2}+\frac{1}{4 H^{2}}\right) \hat{P}
\end{aligned}
$$

Nontrivial solutions:

$$
\begin{aligned}
0=N^{2} \mu\left(e^{-2 i v \tau}-1\right) & \mu L^{2}-\underset{\sim}{i} \frac{1}{2} N^{2} \mu\left(e^{-2 i v \tau}+2 e^{-i v \tau}+1\right) \tau \mu \beta \tilde{\xi}_{k} \\
& +f_{0}^{2}\left[2 \mu^{2}\left(e^{-i v \tau}-1\right)+\left(e^{-i v \tau}-1\right)^{2}\right]\left(m^{2}+\frac{1}{4 H^{2}}\right)
\end{aligned}
$$

Arranging the terms of (Ro.12): 


$$
\begin{aligned}
0= & N^{2} \mu^{2} L^{2} e^{-2 i v \tau}-\underset{\sim}{i} \frac{1}{2} \tau N^{2} \mu^{2} \beta \tilde{\xi} k e^{-2 i v \tau}-\underset{\sim}{i} \tau N^{2} \mu^{2} \beta \tilde{\xi} k e^{-i v \tau}-\underset{\sim}{i} \frac{1}{2} \tau N^{2} \mu^{2} \beta \tilde{\xi} k-N^{2} \mu^{2} L^{2} \\
& +f_{0}^{2}\left(m^{2}+\frac{1}{4 H^{2}}\right) e^{-2 i v \tau}+2\left(\mu^{2}-1\right) f_{0}^{2}\left(m^{2}+\frac{1}{4 H^{2}}\right) e^{-i v \tau}+\left(1-2 \mu^{2}\right) f_{0}^{2}\left(m^{2}+\frac{1}{4 H^{2}}\right)
\end{aligned}
$$

Rearranging the terms in (Ro.13):

$$
\begin{aligned}
0=\left[N^{2} \mu^{2} L^{2}+\right. & \left.f_{0}^{2}\left(m^{2}+\frac{1}{4 H^{2}}\right)\right] e^{-2 \underset{\nu}{i} \tau}-\underset{\sim}{i} \frac{1}{2} \tau N^{2} \mu^{2} \beta \tilde{\xi} k e^{-2 i v \tau} \\
-\underset{\sim}{i} \tau N^{2} & \mu^{2} \beta \tilde{\xi} k e^{-i v \tau}+2\left(\mu^{2}-1\right) f_{0}^{2}\left(m^{2}+\frac{1}{4 H^{2}}\right) e^{-i v \tau} \\
& -\underset{\sim}{i} \frac{1}{2} \tau N^{2} \mu^{2} \beta \tilde{\xi} k+\left(1-2 \mu^{2}\right) f_{0}^{2}\left(m^{2}+\frac{1}{4 H^{2}}\right)-N^{2} \mu^{2} L^{2}
\end{aligned}
$$

Defining:

$$
e^{-i v \tau}=e^{v_{i} \tau}\left[\cos \left(v_{r} \tau\right)-\underset{\sim}{i} \sin \left(v_{r} \tau\right)\right]
$$

Using (Ro.15) in (Ro.14):

$$
\begin{array}{r}
0=\left[N^{2} \mu^{2} L^{2}+f_{0}^{2}\left(m^{2}+\frac{1}{4 H^{2}}\right)\right] e^{2 v_{i} \tau}\left[\cos \left(2 v_{r} \tau\right)-\underset{\sim}{i} \sin \left(2 v_{r} \tau\right)\right]-\underset{\sim}{i} \frac{1}{2} \tau N^{2} \mu^{2} \beta \tilde{\xi} k e^{2 v_{i} \tau}\left[\cos \left(2 v_{r} \tau\right)-\underset{\sim}{i} \sin \left(2 v_{r} \tau\right)\right] \\
-\underset{\sim}{i} \tau N^{2} \mu^{2} \beta \tilde{\xi}_{k} k e^{v_{i} \tau}\left[\cos \left(v_{r} \tau\right)-\underset{\sim}{i} \sin \left(v_{r} \tau\right)\right]+2\left(\mu^{2}-1\right) f_{0}^{2}\left(m^{2}+\frac{1}{4 H^{2}}\right) e^{v_{i} \tau}\left[\cos \left(v_{r} \tau\right)-\underset{\sim}{i} \sin \left(v_{r} \tau\right)\right] \\
-\underset{\sim}{i} \frac{1}{2} \tau N^{2} \mu^{2} \beta \tilde{\xi}_{k}+\left(1-2 \mu^{2}\right) f_{0}^{2}\left(m^{2}+\frac{1}{4 H^{2}}\right)-N^{2} \mu^{2} L^{2}
\end{array}
$$

Arranging terms:

$$
\begin{array}{r}
0=\left[N^{2} \mu^{2} L^{2}+f_{0}^{2}\left(m^{2}+\frac{1}{4 H^{2}}\right)\right] e^{2 v_{i} \tau} \cos \left(2 v_{r} \tau\right)-\underset{\sim}{i} \frac{1}{2} \tau N^{2} \mu^{2} \beta \tilde{\xi}_{k e^{2 v_{i} \tau}} \cos \left(2 v_{r} \tau\right) \\
-\underset{\sim}{i} \tau N^{2} \mu^{2} \beta \tilde{\xi} k e^{v_{i} \tau} \cos \left(v_{r} \tau\right)+2\left(\mu^{2}-1\right) f_{0}^{2}\left(m^{2}+\frac{1}{4 H^{2}}\right) e^{v_{i} \tau} \cos \left(v_{r} \tau\right) \\
-\underset{\sim}{i} \frac{1}{2} \tau N^{2} \mu^{2} \beta \tilde{\xi}_{k}+\left(1-2 \mu^{2}\right) f_{0}^{2}\left(m^{2}+\frac{1}{4 H^{2}}\right)-N^{2} \mu^{2} L^{2}
\end{array}
$$




$$
\begin{aligned}
-i\left[N^{2} \mu^{2} L^{2}\right. & \left.+f_{0}^{2}\left(m^{2}+\frac{1}{4 H^{2}}\right)\right] e^{2 v_{i} \tau} \sin \left(2 v_{r} \tau\right)-\frac{1}{2} \tau N^{2} \mu^{2} \beta \tilde{\xi}_{k} k e^{2 v_{i} \tau} \sin \left(2 v_{r} \tau\right) \\
& -\tau N^{2} \mu^{2} \beta \tilde{\xi} k e^{v_{i} \tau} \sin \left(v_{r} \tau\right)-\underset{\sim}{i}\left(\mu^{2}-1\right) f_{0}^{2}\left(m^{2}+\frac{1}{4 H^{2}}\right) e^{v_{i} \tau} \sin \left(v_{r} \tau\right)
\end{aligned}
$$

Rearranging terms:

$$
\begin{aligned}
& 0=\left[N^{2} \mu^{2} L^{2}+f_{0}^{2}\left(m^{2}+\frac{1}{4 H^{2}}\right)\right] e^{2 v_{i} \tau} \cos \left(2 v_{r} \tau\right)+2\left(\mu^{2}-1\right) f_{0}^{2}\left(m^{2}+\frac{1}{4 H^{2}}\right) e^{v_{i} \tau} \cos \left(v_{r} \tau\right)
\end{aligned}
$$

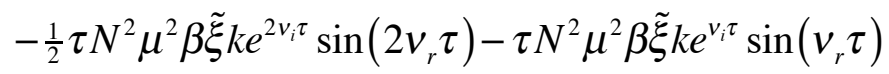

$$
\begin{aligned}
& +\left(1-2 \mu^{2}\right) f_{0}^{2}\left(m^{2}+\frac{1}{4 H^{2}}\right)-N^{2} \mu^{2} L^{2} \\
& -\underset{\sim}{i} \tau N^{2} \mu^{2} \beta \tilde{\xi} k e^{v_{i} \tau} \cos \left(v_{r} \tau\right)-\underset{\sim}{i} \frac{1}{2} \tau N^{2} \mu^{2} \beta \tilde{\xi}_{k} e^{2 v_{i} \tau} \cos \left(2 v_{r} \tau\right)-\underset{\sim}{i} \frac{1}{2} \tau N^{2} \mu^{2} \beta \tilde{\xi}_{k} \\
& -i\left[N^{2} \mu^{2} L^{2}+f_{0}^{2}\left(m^{2}+\frac{1}{4 H^{2}}\right)\right] e^{2 v_{i} \tau} \sin \left(2 v_{r} \tau\right)-\underset{\sim}{i} 2\left(\mu^{2}-1\right) f_{0}^{2}\left(m^{2}+\frac{1}{4 H^{2}}\right) e^{v_{i} \tau} \sin \left(v_{r} \tau\right)
\end{aligned}
$$

Then, two equations are obtained:

$$
\begin{aligned}
& {\left[N^{2} \mu^{2} L^{2}+f_{0}^{2}\left(m^{2}+\frac{1}{4 H^{2}}\right)\right] e^{2 v_{i} \tau} \cos \left(2 v_{r} \tau\right)+2\left(\mu^{2}-1\right) f_{0}^{2}\left(m^{2}+\frac{1}{4 H^{2}}\right) e^{v_{i} \tau} \cos \left(v_{r} \tau\right)} \\
& -\frac{1}{2} \tau N^{2} \mu^{2} \beta \tilde{\xi}_{k e^{2 v_{i} \tau}} \sin \left(2 v_{r} \tau\right)-\tau N^{2} \mu^{2} \beta \tilde{\xi}_{k e^{v_{i} \tau}} \sin \left(v_{r} \tau\right)+\left(1-2 \mu^{2}\right) f_{0}^{2}\left(m^{2}+\frac{1}{4 H^{2}}\right)-N^{2} \mu^{2} L^{2}=0
\end{aligned}
$$

and

$$
\begin{gathered}
\tau N^{2} \mu^{2} \beta \tilde{\xi}_{k} e^{v_{i} \tau} \cos \left(v_{r} \tau\right)+\frac{1}{2} \tau N^{2} \mu^{2} \beta \tilde{\xi}_{k} e^{2 v_{i} \tau} \cos \left(2 v_{r} \tau\right)+\frac{1}{2} \tau N^{2} \mu^{2} \beta \tilde{\xi}_{k} \\
+\left[N^{2} \mu^{2} L^{2}+f_{0}^{2}\left(m^{2}+\frac{1}{4 H^{2}}\right)\right] e^{2 v_{i} \tau} \sin \left(2 v_{r} \tau\right)+2\left(\mu^{2}-1\right) f_{0}^{2}\left(m^{2}+\frac{1}{4 H^{2}}\right) e^{v_{i} \tau} \sin \left(v_{r} \tau\right)=0
\end{gathered}
$$

Using $\sigma_{m}^{2} \equiv m^{2}+\frac{1}{4 H^{2}}$ in (Ro.19a) and (Ro.19b):

$$
\begin{aligned}
& \left(\mu^{2} N^{2} L^{2}+f_{0}^{2} \sigma_{m}^{2}\right) e^{2 v_{i} \tau} \cos \left(2 v_{r} \tau\right)+2\left(\mu^{2}-1\right) f_{0}^{2} \sigma_{m}^{2} e^{v_{i} \tau} \cos \left(v_{r} \tau\right) \\
& -\frac{1}{2} \tau N^{2} \mu^{2} \beta \tilde{\xi} k e^{2 v_{i} \tau} \sin \left(2 v_{r} \tau\right)-\tau N^{2} \mu^{2} \beta \tilde{\xi} k e^{v_{i} \tau} \sin \left(v_{r} \tau\right)+\left(1-2 \mu^{2}\right) f_{0}^{2} \sigma_{m}^{2}-N^{2} \mu^{2} L^{2}=0
\end{aligned}
$$

and 


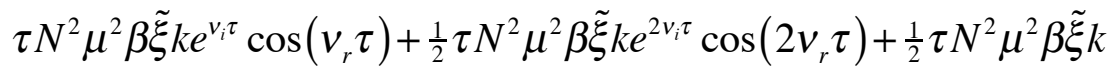

$$
\begin{aligned}
& +\left(\mu^{2} N^{2} L^{2}+f_{0}^{2} \sigma_{m}^{2}\right) e^{2 v_{i} \tau} \sin \left(2 v_{r} \tau\right)+2\left(\mu^{2}-1\right) f_{0}^{2} \sigma_{m}^{2} e^{v_{i} \tau} \sin \left(v_{r} \tau\right)=0
\end{aligned}
$$

Rewriting (Ro.20a):

$$
\begin{aligned}
& a e^{2 v_{i} \tau}+b e^{v_{i} \tau}+c=0 \\
& a \equiv\left(N^{2} \mu^{2} L^{2}+f_{0}^{2} \sigma_{m}^{2}\right) \cos \left(2 v_{r} \tau\right)-\frac{1}{2} \tau N^{2} \mu^{2} \beta \tilde{\xi}_{k} \sin \left(2 v_{r} \tau\right) \\
& b \equiv 2\left(\mu^{2}-1\right) f_{0}^{2} \sigma_{m}^{2} \cos \left(v_{r} \tau\right)-\tau N^{2} \mu^{2} \beta \tilde{\xi}_{k} \sin \left(v_{r} \tau\right) \\
& c \equiv\left(1-2 \mu^{2}\right) f_{0}^{2} \sigma_{m}^{2}-N^{2} \mu^{2} L^{2}
\end{aligned}
$$

Roots of (Ro.21.a)

$$
e^{v_{i} \tau}=\frac{-b+\sqrt{b^{2}-4 a c}}{2 a} \quad \text { (Positive root is selected because } \tau \rightarrow 0, e^{v_{i} \tau} \rightarrow 1 \text { ) (Ro.21.a.a) }
$$


FINAL SOLUTION (Newton-Raphson method):

$$
\begin{aligned}
& e^{v_{i} \tau}=\frac{-b+\sqrt{b^{2}-4 a c}}{2 a} \\
& a \equiv \mu^{2} N^{2} \cos \left(2 v_{r} \tau\right) L^{2}-\frac{1}{2} \mu^{2} N^{2} \sin \left(2 v_{r} \tau\right) \tau \beta \tilde{\xi}_{k}+f_{0}^{2} \cos \left(2 v_{r} \tau\right) \sigma_{m}^{2} \\
& b \equiv 2 f_{0}^{2}\left(\mu^{2}-1\right) \cos \left(v_{r} \tau\right) \sigma_{m}^{2}-\mu^{2} N^{2} \sin \left(v_{r} \tau\right) \tau \beta \tilde{\xi}_{k} \\
& c \equiv f_{0}^{2}\left(1-2 \mu^{2}\right) \sigma_{m}^{2}-\mu^{2} N^{2} L^{2} \\
& \frac{\partial a}{\partial v_{r}} \equiv-2 \tau \mu^{2} N^{2} \sin \left(2 v_{r} \tau\right) L^{2}-\mu^{2} N^{2} \tau^{2} \cos \left(2 v_{r} \tau\right) \beta \tilde{\xi}_{k}-2 \tau f_{0}^{2} \sin \left(2 v_{r} \tau\right) \sigma_{m}^{2} \\
& \frac{\partial b}{\partial v_{r}} \equiv-2 \tau f_{0}^{2}\left(\mu^{2}-1\right) \sin \left(v_{r} \tau\right) \sigma_{m}^{2}-\mu^{2} N^{2} \tau^{2} \cos \left(v_{r} \tau\right) \beta \tilde{\xi}_{k}
\end{aligned}
$$$$
\frac{\partial e^{v_{i} \tau}}{\partial v_{r}}=\frac{a\left[-\frac{\partial b}{\partial v_{r}}+\frac{1}{2}\left(b^{2}-4 a c\right)^{-1 / 2}\left(2 b \frac{\partial b}{\partial v_{r}}-4 c \frac{\partial a}{\partial v_{r}}\right)\right]-\frac{\partial a}{\partial v_{r}}\left[-b+\left(b^{2}-4 a c\right)^{1 / 2}\right]}{2 a^{2}}
$$

$$
\begin{aligned}
F\left(v_{r}\right) \equiv\left[\mu^{2} N^{2} \sin \left(2 v_{r} \tau\right) L^{2}+\frac{1}{2} \mu^{2} N^{2} \cos \left(2 v_{r} \tau\right) \tau \beta \tilde{\xi}_{k}+f_{0}^{2} \sin \left(2 v_{r} \tau\right) \sigma_{m}^{2}\right] e^{2 v_{i} \tau} \\
+\left[\mu^{2} N^{2} \cos \left(v_{r} \tau\right) \tau \beta \tilde{\xi}_{k}+2 f_{0}^{2}\left(\mu^{2}-1\right) \sin \left(v_{r} \tau\right) \sigma_{m}^{2}\right] e^{v_{i} \tau}+\frac{1}{2} \mu^{2} N^{2} \tau \beta \tilde{\xi}_{k}
\end{aligned}
$$

$\frac{\partial F}{\partial \nu_{r}}=\left[2 \tau \mu^{2} N^{2} L^{2} \cos \left(2 v_{r} \tau\right)-\mu^{2} N^{2} \tau^{2} \beta \tilde{\xi}_{k} \sin \left(2 v_{r} \tau\right)+2 \tau f_{0}^{2} \sigma_{m}^{2} \cos \left(2 v_{r} \tau\right)\right] e^{2 v_{i} \tau}$

$$
\begin{aligned}
& +2\left[\mu^{2} N^{2} L^{2} \sin \left(2 v_{r} \tau\right)+\frac{1}{2} \mu^{2} N^{2} \tau \beta \tilde{\xi} k \cos \left(2 v_{r} \tau\right)+f_{0}^{2} \sigma_{m}^{2} \sin \left(2 v_{r} \tau\right)\right] e^{v_{i} \tau} \frac{\partial e^{v_{i} \tau}}{\partial v_{r}} \\
& +\left[-\mu^{2} N^{2} \tau^{2} \sin \left(v_{r} \tau\right) \beta \tilde{\xi}_{k}+2 \tau f_{0}^{2}\left(\mu^{2}-1\right) \sigma_{m}^{2} \cos \left(v_{r} \tau\right)\right] e^{v_{i} \tau} \\
& +\left[\mu^{2} N^{2} \cos \left(v_{r} \tau\right) \tau \beta \tilde{\xi}_{k}+2 f_{0}^{2}\left(\mu^{2}-1\right) \sin \left(v_{r} \tau\right) \sigma_{m}^{2}\right] \frac{\partial e^{v_{i} \tau}}{\partial v_{r}}
\end{aligned}
$$

$$
\left(v_{r}\right)_{1}=\left(v_{r}\right)_{0}-\frac{F\left[\left(v_{r}\right)_{0}\right]}{\left(\frac{\partial F}{\partial v_{r}}\right)_{0}}
$$




\section{Barotropic mode:}

Rewriting equations (RC.13)-(RD.17) with $\mathrm{D}=0(\mathrm{w}=0)$ :

Predictor step on the C-grid:

$\hat{\omega}_{z}^{(*)}=\mu \hat{\omega}_{z}^{(\mathrm{n})}-\underset{\sim}{i} \frac{1}{2} \tau \mu \frac{\beta}{f_{0}} \tilde{\xi}_{k} \hat{P}$
Corrector step on the D-grid:

$\hat{\omega}_{z}^{(\mathrm{n}+1)}=\hat{\omega}_{z}^{(\mathrm{n})}-\underset{\sim}{i \tau} \frac{\beta}{f_{0}} \tilde{\xi}_{k} \hat{P}$

(RBtpcD.1)

$0=f_{0} \hat{\boldsymbol{\omega}}_{z}^{(*)}+\mu L^{2} \hat{P}$

Writing (RBtpcD.1) without changing:

$$
\hat{\omega}_{z}^{(\mathrm{n}+1)}=\hat{\omega}_{z}^{(\mathrm{n})}-\underset{\sim}{i \tau} \frac{\beta}{f_{0}} \tilde{\xi}_{k} \hat{P}
$$

Using (RBtpcC.1) in (RBtpcD.2):

$$
0=\mu f_{0} \hat{\omega}_{z}^{(\mathrm{n})}+\mu\left(L^{2}-\underset{\sim}{i} \frac{1}{2} \tau \beta \tilde{\xi}_{k}\right) \hat{P}
$$

Using $\hat{\Phi}^{(\mathrm{n}+1)}=e^{-i v \tau} \hat{\Phi}^{(\mathrm{n})}$ in (RBtpcCD.1) and (RBtpcCD.2), and dropping the time stamp (n):

$$
\left(e^{i v \tau}-1\right) \hat{\omega}_{z}=-\underset{\sim}{i} \tau \frac{\beta}{f_{0}} \tilde{\xi}_{k} \hat{P}
$$

and

$$
0=\mu f_{0} \hat{\omega}_{z}+\mu\left(L^{2}-\underset{\sim}{i} \frac{1}{2} \tau \beta \tilde{\xi}_{k}\right) \hat{P}
$$

Using (RBtpcCD.3) in (RBtpcCD.4):

$$
\left(e^{i v \tau}-1\right)\left(L^{2}-\underset{\sim}{i} \frac{1}{2} \tau \beta \tilde{\xi}_{k}\right) \hat{P}=\underset{\sim}{i} \tau \beta \tilde{\xi} k \hat{P}
$$

Using $e^{-i v \tau}=e^{v_{i} \tau}\left[\cos \left(v_{r} \tau\right)-\underset{\sim}{i} \sin \left(v_{r} \tau\right)\right]$ in (RBtpcCD.5), and seeking non trivial solutions:

$$
\left[e^{v_{i} \tau} \cos \left(v_{r} \tau\right)-1-\underset{\sim}{i} e^{v_{i} \tau} \sin \left(v_{r} \tau\right)\right]\left(L^{2}-\underset{\sim}{i} \frac{1}{2} \tau \beta \tilde{\xi}_{k}\right)=\underset{\sim}{i} \tau \beta \tilde{\xi} k
$$


Arranging the terms of (RBtpcCD.6):

$$
\left[e^{v_{i} \tau} \cos \left(v_{r} \tau\right)-1\right] L^{2}-e^{v_{i} \tau} \sin \left(v_{r} \tau\right) \frac{1}{2} \tau \beta \tilde{\xi}_{k}-\underset{\sim}{i}\left[e^{v_{i} \tau} \cos \left(v_{r} \tau\right)-1\right] \frac{1}{2} \tau \beta \tilde{\xi}_{k}-\underset{\sim}{i} e^{v_{i} \tau} \sin \left(v_{r} \tau\right) L^{2}-\underset{\sim}{i} \tau \beta \tilde{\xi}_{k}=0
$$

(RBtpcCD.7)

Rearranging the terms of (RBtpcCD.7):

$$
\left[e^{v_{i} \tau} \cos \left(v_{r} \tau\right)-1\right] L^{2}-e^{v_{i} \tau} \sin \left(v_{r} \tau\right) \frac{1}{2} \tau \beta \tilde{\xi}_{k}+\underset{\sim}{i}\left\{-\left[e^{v_{i} \tau} \cos \left(v_{r} \tau\right)+1\right] \frac{1}{2} \tau \beta \tilde{\xi}_{k}-e^{v_{i} \tau} \sin \left(v_{r} \tau\right) L^{2}\right\}=0
$$

(RBtpcCD.7)

Splitting the real and imaginary parts of (RBtpcCD.7):

$$
\left[e^{\nu_{i} \tau} \cos \left(v_{r} \tau\right)-1\right] L^{2}-e^{v_{i} \tau} \sin \left(v_{r} \tau\right) \frac{1}{2} \tau \beta \tilde{\xi}_{k}=0
$$

and

$$
-\left[e^{v_{i} \tau} \cos \left(v_{r} \tau\right)+1\right] \frac{1}{2} \tau \beta \tilde{\xi}_{k}-e^{v_{i} \tau} \sin \left(v_{r} \tau\right) L^{2}=0
$$

Rewriting (RBtpcCD.8a):

$$
e^{v_{i} \tau}=\frac{L^{2}}{L^{2} \cos \left(v_{r} \tau\right)-\sin \left(v_{r} \tau\right) \frac{1}{2} \tau \beta \tilde{\xi}_{k}}
$$

Rewriting (RBtpcCD.8b):

$$
\frac{1}{2} \tau \beta \tilde{\xi}_{k e^{v_{i} \tau}} \cos \left(v_{r} \tau\right)+\frac{1}{2} \tau \beta \tilde{\xi}_{k}+e^{v_{i} \tau} \sin \left(v_{r} \tau\right) L^{2}=0
$$

Using (RBtpcCD.9a) in (RBtpcCD.9b):

$$
\tau \beta \tilde{\xi}_{k L^{2}} \cos \left(v_{r} \tau\right)+\left[\left(L^{2}\right)^{2}-\left(\frac{1}{2} \tau \beta \tilde{\xi}_{k}\right)^{2}\right] \sin \left(v_{r} \tau\right)=0
$$

Rewriting (RBtpcCD.10):

$$
\frac{\sin \left(v_{r} \tau\right)}{\cos \left(v_{r} \tau\right)}=\frac{-\tau \beta \tilde{\xi}_{k L^{2}}}{\left(L^{2}\right)^{2}-\left(\frac{1}{2} \tau \beta \tilde{\xi}_{k}\right)^{2}}
$$

Rewriting (RBtpcCD.11): 


$$
v_{r}=\frac{1}{\tau} \operatorname{Arctan}\left[\frac{-\tau \beta \tilde{\xi}_{k L^{2}}}{\left(L^{2}\right)^{2}-\left(\frac{1}{2} \tau \beta \tilde{\xi}_{k}\right)^{2}}\right]
$$

(a)

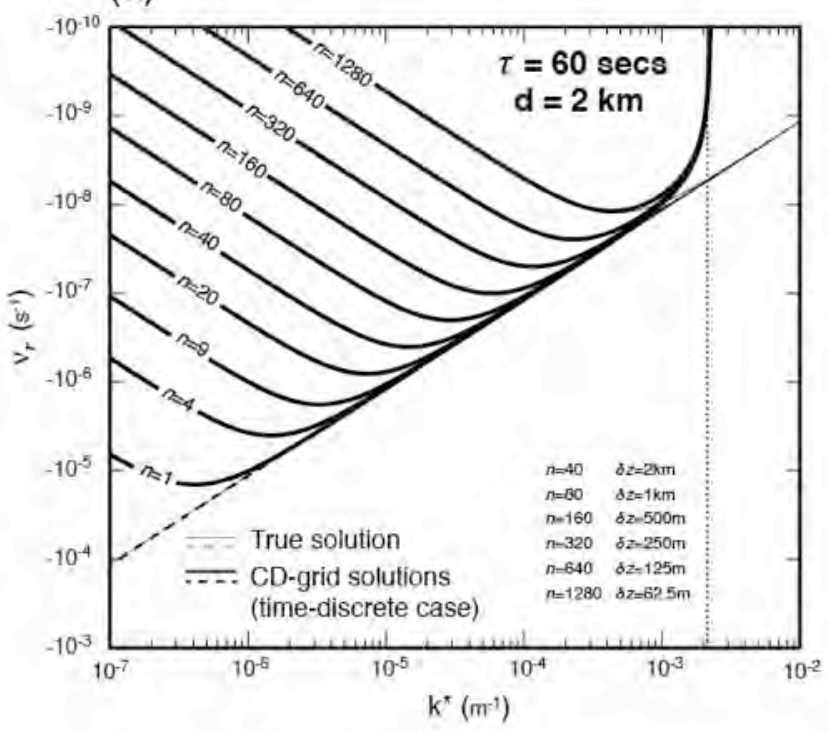

(c)

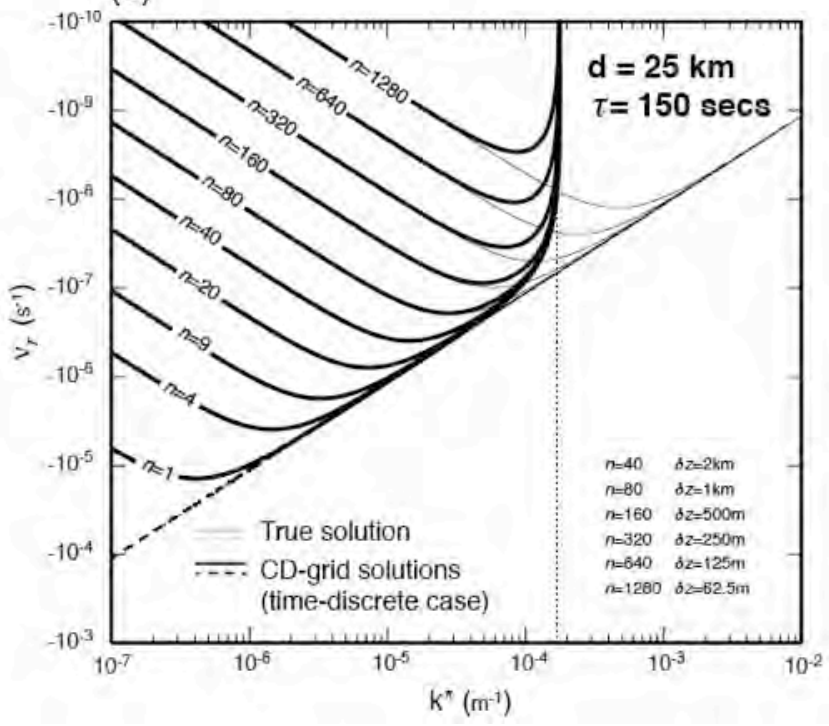

(b)

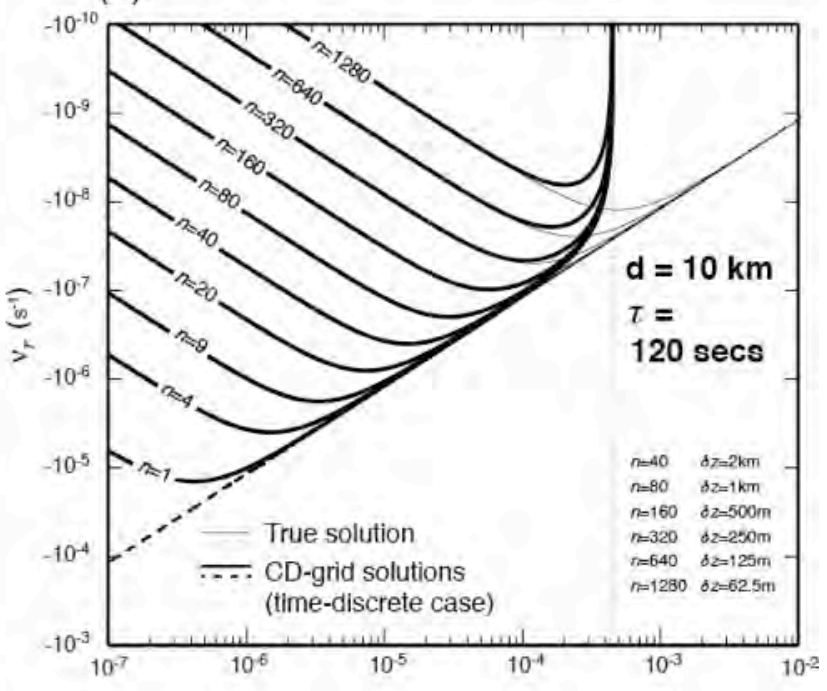

(d)

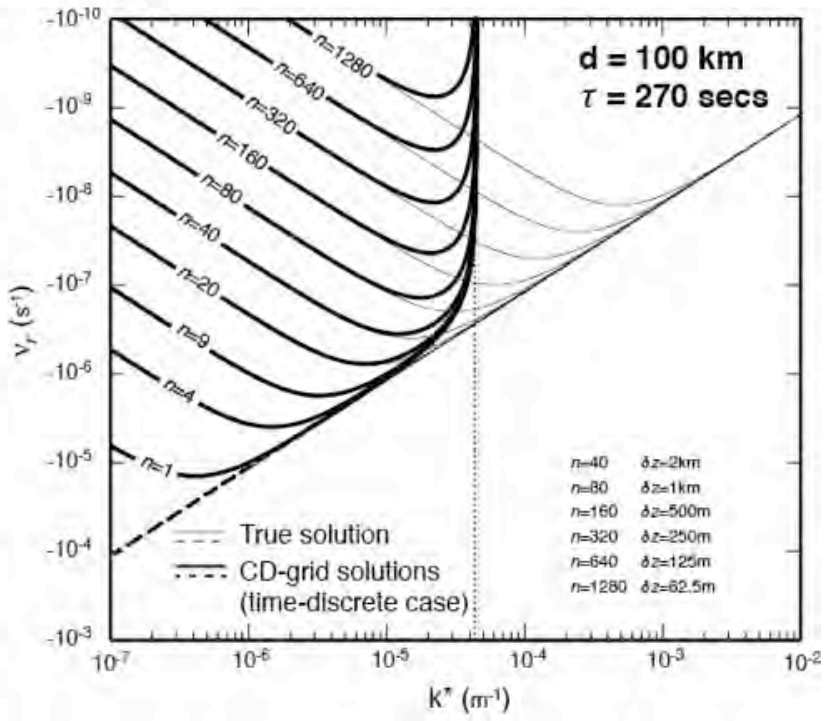

Figure (runs_CD_grid_time_discrete_beta_NEW) 


\section{Scheme R2}

Baroclinic modes:

Predictor-step on the $\mathrm{C}$ grid:

$D$ is diagnostic and local to $C$ grid.

$$
\begin{aligned}
& \hat{\omega}_{z}^{(*)}=\mu \hat{\omega}_{z}^{(\mathrm{n})}-\frac{\tau}{2} \mu f_{0} \hat{D}-\underset{\sim}{i} \frac{\tau}{2} \mu \frac{\beta}{f_{0}} \tilde{\xi}_{k} \hat{P} \\
& 0=f_{0} \hat{\omega}_{z}^{(*)}+L^{2} \hat{P} \\
& 0=-\left(\underset{\sim}{i} m+\frac{1}{2 H}\right) \hat{P}+\hat{B}^{(*)} \\
& \hat{B}^{(*)}=\hat{B}^{(\mathrm{n})}-\frac{\tau}{2} N^{2} \hat{w} \\
& \hat{D}+(\underbrace{i m}_{\sim}-\frac{1}{2 H}) \hat{w}=0
\end{aligned}
$$

Eliminate the diagnostic variables $\mathrm{D}(\mathrm{w})$ and $\mathrm{P}$ between the predictor-step equations.

Using (RS1P.2) in (RS1P.1):

$$
\left(L^{2}-\underset{i}{\sim} \frac{\tau}{2} \mu \frac{\beta}{f_{0}} \tilde{\xi}_{k f_{0}}\right) \hat{\boldsymbol{\omega}}_{z}^{(*)}=\mu L^{2} \hat{\boldsymbol{\omega}}_{z}^{(\mathrm{n})}-\frac{\tau}{2} \mu f_{0} L^{2} \hat{D}
$$

Using (RS1P.3) in (RS1P.2):

$$
0=f_{0}\left(\underset{\sim}{i m}+\frac{1}{2 H}\right) \hat{\boldsymbol{\omega}}_{z}^{(*)}+L^{2} \hat{B}^{(*)}
$$

Using (RS1P.5) in (RS1P.4):

$$
\left(\underset{\sim}{i m}-\frac{1}{2 H}\right) \hat{B}^{(*)}=\left(\underset{\sim}{i m}-\frac{1}{2 H}\right) \hat{B}^{(\mathrm{n})}+\frac{\tau}{2} N^{2} \hat{D}
$$


Eliminating D between (RS1P.6) and (RS1P.8):

$$
N^{2}\left(L^{2}-\underset{\sim}{i} \frac{\tau}{2} \mu \frac{\beta}{f_{0}} \tilde{\xi}_{k f_{0}}\right) \hat{\boldsymbol{\omega}}_{z}^{(*)}+\mu f_{0}\left(\underset{\sim}{i m}-\frac{1}{2 H}\right) L^{2} \hat{B}^{(*)}=\mu N^{2} L^{2} \hat{\boldsymbol{\omega}}_{z}^{(\mathrm{n})}+\mu f_{0}\left(\underset{\sim}{i m}-\frac{1}{2 H}\right) L^{2} \hat{B}^{(\mathrm{n})}
$$

Eliminating $\hat{B}^{(*)}$ between (RS1P.9) and (RS1P.7):

$$
\left[N^{2}\left(L^{2}-\underset{i}{i} \frac{\tau}{2} \mu \frac{\beta}{f_{0}} \tilde{\xi}_{k f_{0}}\right)+\mu f_{0}^{2}\left(m^{2}+\frac{1}{4 H^{2}}\right)\right] \hat{\boldsymbol{\omega}}_{z}^{(*)}=\mu N^{2} L^{2} \hat{\boldsymbol{\omega}}_{z}^{(\mathrm{n})}+\mu f_{0}\left(\underset{\sim}{i m}-\frac{1}{2 H}\right) L^{2} \hat{B}^{(\mathrm{n})}
$$

Eliminating $\hat{\omega}_{z}^{(*)}$ between (RS1P.9) and (RS1P.7):

$$
\left[N^{2}\left(L^{2}-\underset{\sim}{i} \frac{\tau}{2} \mu \frac{\beta}{f_{0}} \tilde{\xi}_{k f_{0}}\right)+\mu f_{0}^{2}\left(m^{2}+\frac{1}{4 H^{2}}\right)\right] \hat{B}^{(*)}=-\mu f_{0} N^{2}\left(\underset{\sim}{i m}+\frac{1}{2 H}\right) \hat{\omega}_{z}^{(\mathrm{n})}+\mu f_{0}^{2}\left(m^{2}+\frac{1}{4 H^{2}}\right) \hat{B}^{(\mathrm{n})}
$$

(RS1P.11)

Corrector-step on the D grid (1):

$$
\begin{aligned}
& \hat{\omega}_{z}^{(\mathrm{n}+1)}=\hat{\omega}_{z}^{(\mathrm{n})}-\tau \mu f_{0} \hat{D}-\underset{\sim}{i} \tau \frac{\beta}{f_{0}} \tilde{\xi}_{k} \hat{P} \\
& 0=f_{0} \hat{\omega}_{z}^{(*)}+\mu L^{2} \hat{P} \\
& 0=-\left(\underset{\sim}{i m}+\frac{1}{2 H}\right) \hat{P}+\hat{B}^{(*)} \\
& \hat{B}^{(\mathrm{n}+1)}=\hat{B}^{(\mathrm{n})}-\tau N^{2} \hat{w} \\
& \mu \hat{D}+\left(\underset{\sim}{i m}-\frac{1}{2 H}\right) \hat{w}=0
\end{aligned}
$$

(RS1P.10) and (RS1C.1.2):

$$
-\left[N^{2}\left(L^{2}-\underset{\sim}{i} \frac{\tau}{2} \mu \frac{\beta}{f_{0}} \tilde{\xi}_{k f_{0}}\right)+\mu f_{0}^{2}\left(m^{2}+\frac{1}{4 H^{2}}\right)\right] \mu \hat{P}=\mu N^{2} f_{0} \hat{\omega}_{z}^{(\mathrm{n})}+\mu f_{0}^{2}\left(\underset{\sim}{i m}-\frac{1}{2 H}\right) \hat{B}^{(\mathrm{n})}
$$

(RS1P.11) and (RS1C.1.3): 


$$
\left[N^{2}\left(L^{2}-\underset{\sim}{i} \frac{\tau}{2} \mu \frac{\beta}{f_{0}} \tilde{\xi}_{k f}\right)+\mu f_{0}^{2}\left(m^{2}+\frac{1}{4 H^{2}}\right)\right]\left(\underset{\sim}{\underset{\sim}{i}} m+\frac{1}{2 H}\right) \hat{P}=-\mu f_{0} N^{2}\left(\underset{\sim}{i} m+\frac{1}{2 H}\right) \hat{\omega}_{z}^{(\mathrm{n})}+\mu f_{0}^{2}\left(m^{2}+\frac{1}{4 H^{2}}\right) \hat{B}^{(\mathrm{n})}
$$

(RS1C.1.7)

Multiplying (RS1C.1.6) by $\left(\underset{\sim}{i} m+\frac{1}{2 H}\right)$ :

$$
-\left[N^{2}\left(L^{2}-\underset{\sim}{i} \frac{\tau}{2} \mu \frac{\beta}{f_{0}} \tilde{\xi}_{k f_{0}}\right)+\mu f_{0}^{2}\left(m^{2}+\frac{1}{4 H^{2}}\right)\right] \mu\left(\underset{\sim}{i} m+\frac{1}{2 H}\right) \hat{P}=\mu N^{2} f_{0}\left(\underset{\sim}{i} m+\frac{1}{2 H}\right) \hat{\omega}_{z}^{(\mathrm{n})}-\mu f_{0}^{2}\left(m^{2}+\frac{1}{4 H^{2}}\right) \hat{B}^{(\mathrm{n})}
$$

(RS1C.1.8)

(RS1C.1.7) and (RS1C.1.8) do not match to each other. No solution for the corrector (1) case.

Corrector-step on the D grid (2):

$$
\begin{aligned}
& \hat{\omega}_{z}^{(\mathrm{n}+1)}=\hat{\omega}_{z}^{(\mathrm{n})}-\tau \mu f_{0} \hat{D}-\underset{\sim}{i} \tau \frac{\beta}{f_{0}} \tilde{\xi}_{k} \hat{P} \\
& 0=\mu f_{0} \hat{\omega}_{z}^{(n+1)}+\mu L^{2} \hat{P} \\
& 0=-\left(\underset{\sim}{i m} m+\frac{1}{2 H}\right) \hat{P}+\hat{B}^{(\mathrm{n}+1)} \\
& \hat{B}^{(\mathrm{n}+1)}=\hat{B}^{(\mathrm{n})}-\tau N^{2} \hat{w} \\
& \mu \hat{D}+\left({\underset{\sim}{i}}^{i m}-\frac{1}{2 H}\right) \hat{w}=0
\end{aligned}
$$

Using $\hat{\Phi}^{(\mathrm{n}+1)}=e^{-i v \tau} \hat{\Phi}^{(\mathrm{n})}$ in (RS1C.2.1)-(RS1C.2.4):

$$
\begin{gathered}
\left(e^{-i v \tau}-1\right) \hat{\omega}_{z}=-\tau \mu f_{0} \hat{D}-\underset{\sim}{i} \tau \frac{\beta}{f_{0}} \tilde{\xi}_{k} \hat{P} \\
0=\mu f_{0} e^{-i v \tau} \hat{\omega}_{z}+\mu L^{2} \hat{P}
\end{gathered}
$$




$$
\begin{gathered}
0=-\left(\underset{\sim}{i} m+\frac{1}{2 H}\right) \hat{P}+e^{-i v \tau} \hat{B} \\
\left(e^{-i v \tau}-1\right) \hat{B}=-\tau N^{2} \hat{w} \\
\mu \hat{D}+\left(\underset{\sim}{i} m-\frac{1}{2 H}\right) \hat{w}=0
\end{gathered}
$$

Eliminating $\hat{\omega}_{z}$ between (RS1C.2.5) and (RS1C.2.6):

$$
\left[\left(e^{-i v \tau}-1\right) L^{2}-\underset{\sim}{i} \tau \beta \tilde{\xi} k e^{-i v \tau}\right] \hat{P}=\tau \mu f_{0}^{2} e^{-i v \tau} \hat{D}
$$

Eliminating w between (RS1C.2.9) and (RS1C.2.8):

$$
\left(e^{-i v \tau}-1\right)\left(\underset{\sim}{i} m-\frac{1}{2 H}\right) \hat{B}=\tau N^{2} \mu \hat{D}
$$

Eliminating B between (RS1C.2.7) and (RS1C.2.11):

$$
0=\left(e^{-i v \tau}-1\right)\left(m^{2}+\frac{1}{4 H^{2}}\right) \hat{P}+e^{-i v \tau} \tau N^{2} \mu \hat{D}
$$

Eliminating D between (RS1C.2.10) and (RS1C.2.12):

$$
N^{2}\left[\left(e^{-i v \tau}-1\right) L^{2}-\underset{\sim}{i} \tau \beta \tilde{\xi} k e^{-i v \tau}\right] \hat{P}=-f_{0}^{2}\left(e^{-i v \tau}-1\right)\left(m^{2}+\frac{1}{4 H^{2}}\right) \hat{P}
$$

Nontrivial solutions and arrange (RS1C.2.13):

$$
\left(e^{-i v \tau}-1\right) N^{2} L^{2}-\underset{\sim}{i} \tau N^{2} \beta \tilde{\xi} k e^{-i v \tau}=-f_{0}^{2}\left(e^{-i v \tau}-1\right)\left(m^{2}+\frac{1}{4 H^{2}}\right)
$$

Rearranging the terms in (RS1C.2.14):

$$
N^{2} L^{2} e^{-i v \tau}+f_{0}^{2}\left(m^{2}+\frac{1}{4 H^{2}}\right) e^{-i v \tau}-\underset{\sim}{i} \tau N^{2} \beta \tilde{\xi} k e^{-i v \tau}=N^{2} L^{2}+f_{0}^{2}\left(m^{2}+\frac{1}{4 H^{2}}\right)
$$

Rearranging the terms in (RS1C.2.15): 


$$
\left[N^{2} L^{2}+f_{0}^{2}\left(m^{2}+\frac{1}{4 H^{2}}\right)-\underset{\sim}{i} \tau N^{2} \beta \tilde{\xi} k\right] e^{-i v \tau}=N^{2} L^{2}+f_{0}^{2}\left(m^{2}+\frac{1}{4 H^{2}}\right)
$$

Using $e^{i v \tau} \equiv e^{v_{i} \tau}\left[\cos \left(v_{r} \tau\right)-\underset{\sim}{i} \sin \left(v_{r} \tau\right)\right]$ in (RS1C.2.16):

$$
\left[N^{2} L^{2}+f_{0}^{2}\left(m^{2}+\frac{1}{4 H^{2}}\right)-\underset{\sim}{i} \tau N^{2} \beta \tilde{\xi} k\right] e^{\nu_{i} \tau}\left[\cos \left(v_{r} \tau\right)-\underset{\sim}{i} \sin \left(v_{r} \tau\right)\right]=N^{2} L^{2}+f_{0}^{2}\left(m^{2}+\frac{1}{4 H^{2}}\right)
$$

(RS1C.2.17)

Rearrange the terms in (RS1C.2.17):

$$
\begin{aligned}
& {\left[N^{2} L^{2}+f_{0}^{2}\left(m^{2}+\frac{1}{4 H^{2}}\right)-\underset{\sim}{i} \tau N^{2} \beta \tilde{\xi} k\right] e^{\nu_{i} \tau} \cos \left(v_{r} \tau\right)} \\
& -\left[N^{2} L^{2}+f_{0}^{2}\left(m^{2}+\frac{1}{4 H^{2}}\right)-\underset{\sim}{i} \tau N^{2} \beta \tilde{\xi} k\right] \underset{\sim}{i} e^{v_{i} \tau} \sin \left(v_{r} \tau\right)=N^{2} L^{2}+f_{0}^{2}\left(m^{2}+\frac{1}{4 H^{2}}\right)
\end{aligned}
$$

Then,

$$
\begin{aligned}
& {\left[N^{2} L^{2}+f_{0}^{2}\left(m^{2}+\frac{1}{4 H^{2}}\right)\right] e^{v_{i} \tau} \cos \left(v_{r} \tau\right)-\underset{\sim}{i} \tau N^{2} \beta \tilde{\xi} k e^{v_{i} \tau} \cos \left(v_{r} \tau\right)} \\
& -\underset{\sim}{i}\left[N^{2} L^{2}+f_{0}^{2}\left(m^{2}+\frac{1}{4 H^{2}}\right)\right] e^{v_{i} \tau} \sin \left(v_{r} \tau\right)-\tau N^{2} \beta \tilde{\xi} k e^{v_{i} \tau} \sin \left(v_{r} \tau\right)=N^{2} L^{2}+f_{0}^{2}\left(m^{2}+\frac{1}{4 H^{2}}\right)
\end{aligned}
$$

(RS1C.2.19)

Then,

$$
\begin{aligned}
& {\left[N^{2} L^{2}+f_{0}^{2}\left(m^{2}+\frac{1}{4 H^{2}}\right)\right] e^{v_{i} \tau} \cos \left(v_{r} \tau\right)} \\
& -\underset{\sim}{i} \tau N^{2} \beta \tilde{\xi} \tilde{\xi}_{k}^{v_{i} \tau} \cos \left(v_{r} \tau\right)-\underset{\sim}{i}\left[N^{2} L^{2}+f_{0}^{2}\left(m^{2}+\frac{1}{4 H^{2}}\right)\right] e^{v_{i} \tau} \sin \left(v_{r} \tau\right) \\
& -\tau N^{2} \beta \tilde{\xi} k e^{v_{i} \tau} \sin \left(v_{r} \tau\right)=N^{2} L^{2}+f_{0}^{2}\left(m^{2}+\frac{1}{4 H^{2}}\right)
\end{aligned}
$$

Separating the real and imaginary parts:

$$
\left[N^{2} L^{2}+f_{0}^{2}\left(m^{2}+\frac{1}{4 H^{2}}\right)\right] e^{v_{i} \tau} \cos \left(v_{r} \tau\right)-\tau N^{2} \beta \tilde{\xi} k e^{v_{i} \tau} \sin \left(v_{r} \tau\right)=N^{2} L^{2}+f_{0}^{2}\left(m^{2}+\frac{1}{4 H^{2}}\right)
$$

(RS1C.2.21a) 
and

$-\underset{\sim}{i} \tau N^{2} \beta \tilde{\xi}_{k} e^{v_{i} \tau} \cos \left(v_{r} \tau\right)-\underset{\sim}{i}\left[N^{2} L^{2}+f_{0}^{2}\left(m^{2}+\frac{1}{4 H^{2}}\right)\right] e^{v_{i} \tau} \sin \left(v_{r} \tau\right)=0$

The dispersion relation:

$$
\frac{\sin \left(v_{r} \tau\right)}{\cos \left(v_{r} \tau\right)}=\frac{-\tau \beta \tilde{\xi}_{k}}{L^{2}+\frac{f_{0}^{2}}{N^{2}}\left(m^{2}+\frac{1}{4 H^{2}}\right)}
$$

and

$$
e^{v_{i} \tau}=\frac{L^{2}+\frac{f_{0}^{2}}{N^{2}}\left(m^{2}+\frac{1}{4 H^{2}}\right)}{\left[L^{2}+\frac{f_{0}^{2}}{N^{2}}\left(m^{2}+\frac{1}{4 H^{2}}\right)\right] \cos \left(v_{r} \tau\right)-\tau \beta \tilde{\xi}_{k} \sin \left(v_{r} \tau\right)}
$$

Rewriting (RS1C.2.22a)

$$
v_{r}=\frac{1}{\tau} \operatorname{Arctan}\left[\frac{-\tau \beta \tilde{\xi}_{k}}{L^{2}+\frac{f_{0}^{2}}{N^{2}}\left(m^{2}+\frac{1}{4 H^{2}}\right)}\right]
$$
dispersion_run2_CD_grid_discrete_beta_2_NEW_Scheme_2_2.f90

FINAL SOLUTION (Newton-Raphson method):

$$
\begin{aligned}
& F\left(v_{r}\right)=\frac{\sin \left(v_{r} \tau\right)}{\cos \left(v_{r} \tau\right)}+\frac{\tau \beta \tilde{\xi}_{k}}{N^{2} L^{2}+\frac{f_{0}^{2}}{N^{2}}\left(m^{2}+\frac{1}{4 H^{2}}\right)} \\
& \frac{\partial F}{\partial v_{r}}=\frac{\tau \cos ^{2}\left(v_{r} \tau\right)+\tau \sin ^{2}\left(v_{r} \tau\right)}{\cos ^{2}\left(v_{r} \tau\right)}=\frac{\tau}{\cos ^{2}\left(v_{r} \tau\right)} \\
& \left(v_{r}\right)_{1}=\left(v_{r}\right)_{0}-\frac{F\left[\left(v_{r}\right)_{0}\right]}{\left(\frac{\partial F}{\partial v_{r}}\right)_{0}}
\end{aligned}
$$




\section{Barotropic modes:}

From (RS1C.2.1) and (RS1C.2.2) without divergence:

$\hat{\omega}_{z}^{(\mathrm{n}+1)}=\hat{\omega}_{z}^{(\mathrm{n})}-\underset{\sim}{i \tau} \frac{\beta}{f_{0}} \tilde{\xi}_{k} \hat{P}$

and

$0=\mu f_{0} \hat{\omega}_{z}^{(n+1)}+\mu L^{2} \hat{P}$

Using $\hat{\Phi}^{(\mathrm{n}+1)}=e^{-i v \tau} \hat{\Phi}^{(\mathrm{n})}$ in (RS1C.2.24)-(RS1C.2.25):

$$
\left(e^{-i v \tau}-1\right) \hat{\omega}_{z}=-\underset{\sim}{i} \tau \frac{\beta}{f_{0}} \tilde{\xi}_{k} \hat{P}
$$

and

$$
0=\mu f_{0} e^{-i v \tau} \hat{\omega}_{z}+\mu L^{2} \hat{P}
$$

Using (RS1C.2.26) in (RS1C.2.27):

$$
\left(e^{-i v \tau}-1\right) L^{2} \hat{P}=\underset{\sim}{i} \tau f_{0} \frac{\beta}{f_{0}} \tilde{\xi}_{k e^{-i v \tau}} \hat{P}
$$

Nontrivial solutions:

$$
e^{-i v \tau} L^{2}-\underset{\sim}{i} \tau f_{0} \frac{\beta}{f_{0}} \tilde{\xi}_{k} e^{-i v \tau}-L^{2}=0
$$

Using $e^{-i v \tau}=e^{v_{i} \tau}\left[\cos \left(v_{r} \tau\right)-\underset{\sim}{i} \sin \left(v_{r} \tau\right)\right]$ in (RS1C.2.29):

$$
L^{2} e^{v_{i} \tau} \cos \left(v_{r} \tau\right)-\tau \beta \tilde{\xi} k e^{v_{i} \tau} \sin \left(v_{r} \tau\right)-L^{2}-\underset{\sim}{i} L^{2} e^{v_{i} \tau} \sin \left(v_{r} \tau\right)-\underset{\sim}{i} \tau \beta \tilde{\xi} k e^{v_{i} \tau} \cos \left(v_{r} \tau\right)=0
$$

Splitting the real and imaginary parts:

$$
L^{2} e^{v_{i} \tau} \cos \left(v_{r} \tau\right)-\tau \beta \tilde{\xi} k e^{v_{i} \tau} \sin \left(v_{r} \tau\right)-L^{2}=0
$$

and

$$
\frac{\sin \left(v_{r} \tau\right)}{\cos \left(v_{r} \tau\right)}=\frac{-\tau \beta \tilde{\xi}_{k}}{L^{2}}
$$


Rewrite (RS1C.2.31a):

$$
e^{v_{i} \tau}=\frac{L^{2}}{L^{2} e^{v_{i} \tau} \cos \left(v_{r} \tau\right)-\tau \beta \tilde{\xi} k e^{v_{i} \tau} \sin \left(v_{r} \tau\right)}
$$

Rewrite (RS1C.2.31b):

$$
\nu_{r}=\frac{1}{\tau} \operatorname{Arctan}\left(\frac{-\tau \beta \tilde{\xi}_{k}}{L^{2}}\right)
$$

dispersion_run2_CD_grid_discrete_beta_2_NEW_Scheme_2_2_barotropic.f90

(a)

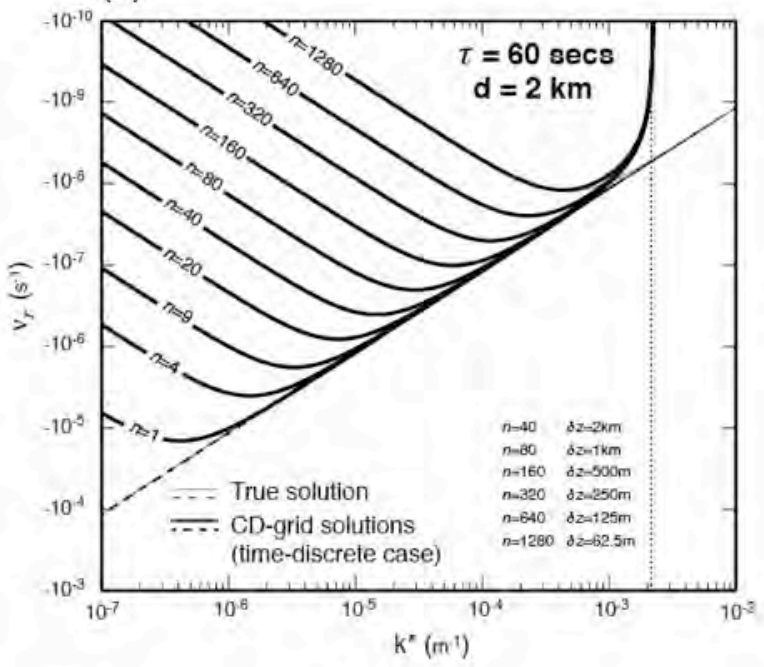

(c)

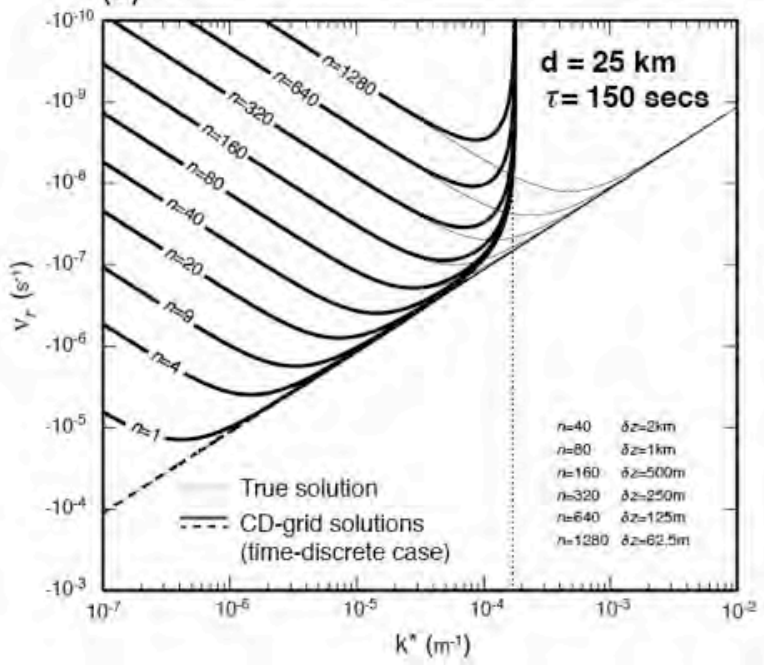

(b)

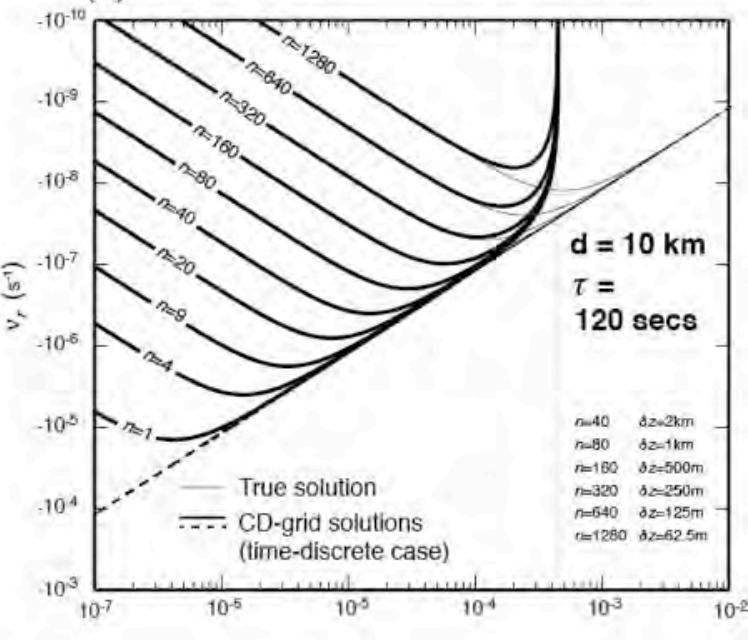

(d)

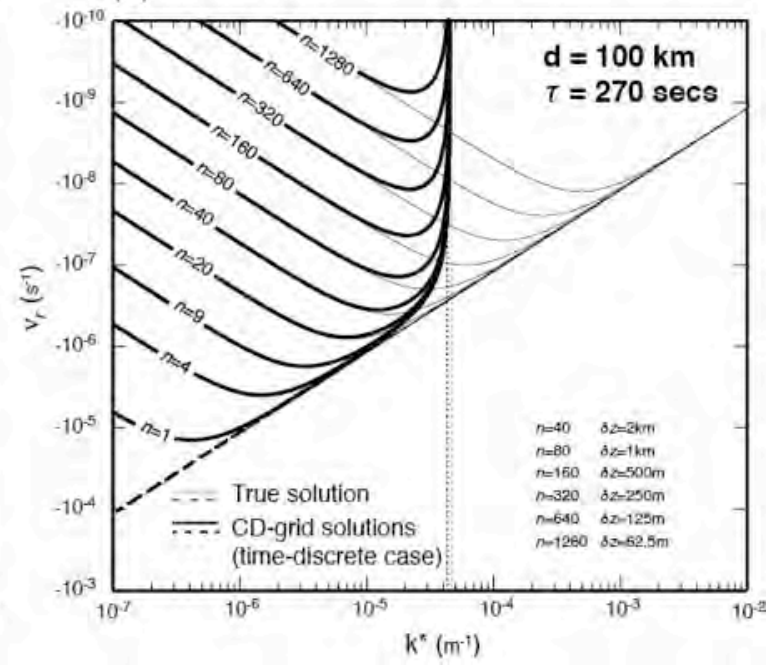

Figure beta scheme II. (runs_CD_grid_time_discrete_beta_NEW_Scheme_2) 


\section{A-grid}

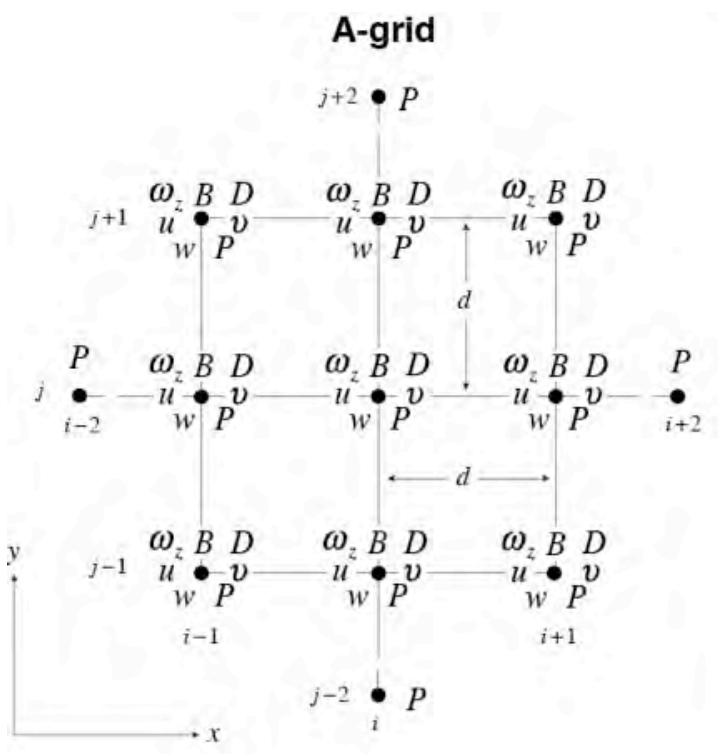

\section{A-grid (Vorticity-divergence point of view)}

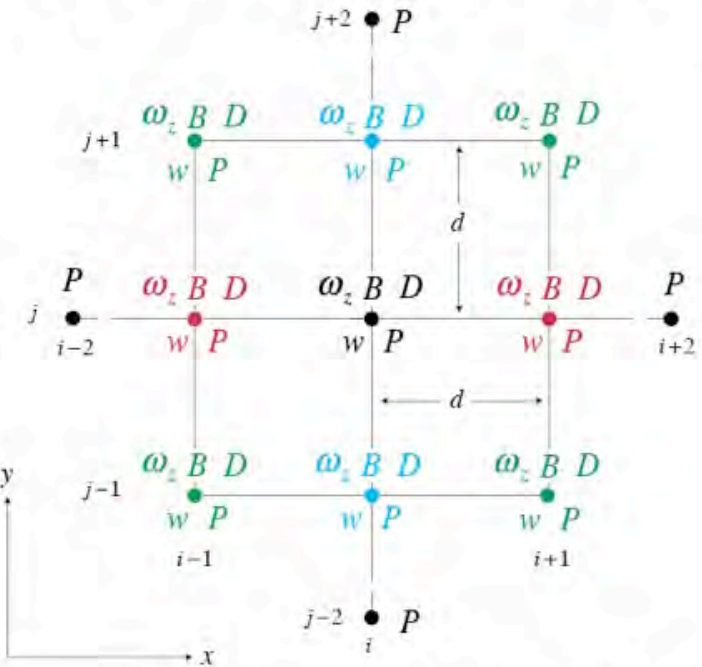

A-grid is a superposition of four independent and noninteracting Z-grids.

\section{A-grid (Combination of C-grids)}

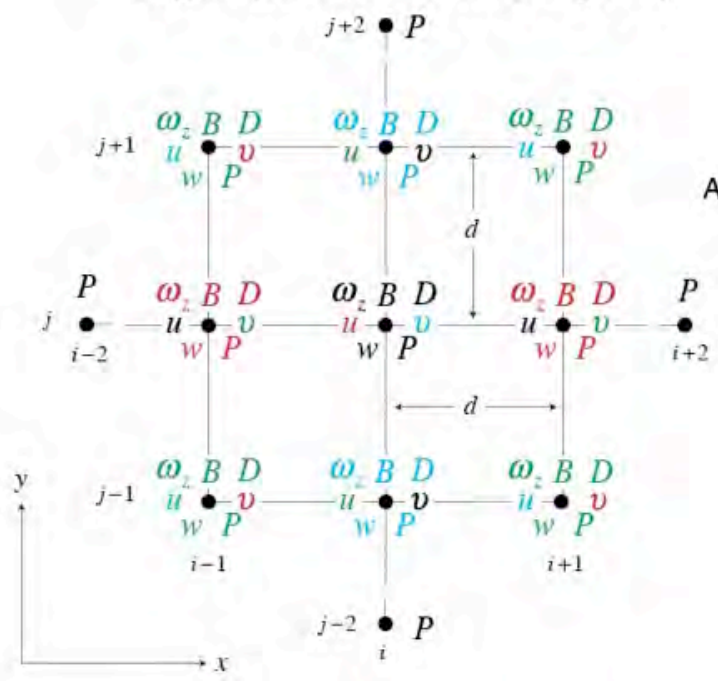

A-grid is a superposition of four C-grids.

A grid

\section{Inertia-gravity modes}

Horizontal momentum equation: 


$$
\begin{gathered}
\frac{\partial u_{i, j}}{\partial t}=-\frac{P_{i+1, j}-P_{i-1, j}}{2 d}+f v_{i, j} \\
\frac{\partial v_{i, j}}{\partial t}=-\frac{P_{i, j+1}-P_{i, j-1}}{2 d}-f u_{i, j}
\end{gathered}
$$

To derive divergence equation for center points $(i, j)$ :

$$
\begin{aligned}
& \frac{\partial}{\partial t}\left(\frac{u_{i+1, j}-u_{i-1, j}}{2 d}\right)=-\frac{P_{i+2, j}+2 P_{i, j}-P_{i-2, j}}{4 d^{2}}+f\left(\frac{v_{i+1, j}-v_{i-1, j}}{2 d}\right) \\
& \frac{\partial}{\partial t}\left(\frac{v_{i, j+1}-v_{i, j-1}}{2 d}\right)=-\frac{P_{i, j+2}+2 P_{i, j}-P_{i, j-2}}{4 d^{2}}-f\left(\frac{u_{i, j+1}-u_{i, j-1}}{2 d}\right)
\end{aligned}
$$

Define:

$$
D_{i, j} \equiv \frac{1}{2 d}\left(u_{i+1, j}-u_{i-1, j}+v_{i, j+1}-v_{i, j-1}\right)
$$

Divergence equation (1):

$$
\frac{\partial}{\partial t}\left(\frac{u_{i+1, j}-u_{i-1, j}}{2 d}+\frac{v_{i, j+1}-v_{i, j-1}}{2 d}\right)=-\frac{P_{i+2, j}+P_{i-2, j}+P_{i, j+2}+P_{i, j-2}-4 P_{i, j}}{4 d^{2}}+f\left(-\frac{u_{i, j+1}-u_{i, j-1}}{2 d}+\frac{v_{i+1, j}-v_{i-1, j}}{2 d}\right)
$$

Define:

$$
\left(\omega_{z}\right)_{i, j} \equiv \frac{1}{2 d}\left(v_{i+1, j}-v_{i-1, j}-u_{i, j+1}+u_{i, j-1}\right)
$$

Divergence equation (2):

Consider (M.6) and (M.7):

$$
\frac{\partial}{\partial t} D_{i, j}=-\frac{P_{i+2, j}+P_{i-2, j}+P_{i, j+2}+P_{i, j-2}-4 P_{i, j}}{4 d^{2}}+f\left(\omega_{z}\right)_{i, j}
$$


To derive vorticity equation for center point $(i, j)$ :

$$
\begin{aligned}
& \frac{\partial}{\partial t}\left(\frac{v_{i+1, j}-v_{i-1, j}}{2 d}\right)=-\frac{P_{i+1, j+1}+P_{i-1, j+1}-P_{i+1, j-1}-P_{i-1, j-1}}{2 d^{2}}-f\left(\frac{u_{i+1, j}-u_{i-1, j}}{2 d}\right) \\
& \frac{\partial}{\partial t}\left(\frac{-u_{i, j+1}+u_{i, j-1}}{2 d}\right)=\frac{P_{i+1, j+1}-P_{i-1, j+1}-P_{i+1, j-1}+P_{i-1, j-1}}{2 d^{2}}-f\left(\frac{v_{i, j+1}-v_{i, j-1}}{2 d}\right)
\end{aligned}
$$

The vorticity equation (1):

$$
\frac{\partial}{\partial t}\left(\frac{v_{i+1, j}-v_{i-1, j}}{2 d}+\frac{-u_{i, j+1}+u_{i, j-1}}{2 d}\right)=-f\left(\frac{u_{i+1, j}-u_{i-1, j}}{2 d}+\frac{v_{i, j+1}-v_{i, j-1}}{2 d}\right)
$$

The vorticity equation (2):

Using (M.7) and (M.5) in (M.11):

$$
\frac{\partial}{\partial t}\left(\omega_{z}\right)_{i, j}=-f D_{i, j}
$$

Vorticity equation from (M.12):

$$
\frac{\partial}{\partial t}\left(\omega_{z}\right)_{i, j}=-f D_{i, j}
$$

Divergence equation from (M.8):

$$
\frac{\partial}{\partial t} D_{i, j}=-\frac{P_{i+2, j}+P_{i-2, j}+P_{i, j+2}+P_{i, j-2}-4 P_{i, j}}{4 d^{2}}+f\left(\omega_{z}\right)_{i, j}
$$

If we use (1) in (2), we obtain

$$
\left(\frac{\partial^{2}}{\partial t^{2}}+f^{2}\right) D_{i, j}=-\frac{\partial}{\partial t}\left(\frac{P_{i+2, j}+P_{i-2, j}+P_{i, j+2}+P_{i, j-2}-4 P_{i, j}}{4 d^{2}}\right)
$$

Vertical momentum equation: 


$$
\frac{\partial w_{i, j}}{\partial t}=-\left[\frac{\partial}{\partial z}-\left(\frac{1}{2 \rho_{0}} \frac{\partial \rho_{0}}{\partial z}\right)\right] P_{i, j}+B_{i, j}
$$

Thermodynamic equation:

$$
\frac{\partial B_{i, j}}{\partial t}=-N^{2} w_{i, j}
$$

Mass continuity equation:

$$
D_{i, j}+\left[\frac{\partial}{\partial z}+\left(\frac{1}{2 \rho_{0}} \frac{\partial \rho_{0}}{\partial z}\right)\right] w_{i, j}=0
$$

From (4)-(6):

$$
\left(\frac{\partial^{2}}{\partial t^{2}}+N^{2}\right) D_{i, j}-\left[\frac{\partial^{2}}{\partial z^{2}}-\left(\frac{1}{2 \rho_{0}} \frac{\partial \rho_{0}}{\partial z}\right)^{2}\right] \frac{\partial}{\partial t} P_{i, j}=0
$$

Using (2') in (7), we obtain the following two equations:

$\left[\frac{\partial^{2}}{\partial z^{2}}-\left(\frac{1}{2 \rho_{0}} \frac{\partial \rho_{0}}{\partial z}\right)^{2}\right]\left(\frac{\partial^{2}}{\partial t^{2}}+f^{2}\right) D_{i, j}=-\left(\frac{\partial^{2}}{\partial t^{2}}+N^{2}\right)\left(\frac{D_{i+2, j}+D_{i-2, j}+D_{i, j+2}+D_{i, j-2}-4 D_{i, j}}{4 d^{2}}\right)$

and

$\left(\frac{\partial^{2}}{\partial t^{2}}+f^{2}\right)\left[\frac{\partial^{2}}{\partial z^{2}}-\left(\frac{1}{2 \rho_{0}} \frac{\partial \rho_{0}}{\partial z}\right)^{2}\right] \frac{\partial}{\partial t} P_{i, j}=-\left(\frac{\partial^{2}}{\partial t^{2}}+N^{2}\right) \frac{\partial}{\partial t}\left(\frac{P_{i+2, j}+P_{i-2, j}+P_{i, j+2}+P_{i, j-2}-4 P_{i, j}}{4 d^{2}}\right)$

This shows that there are four independent and noninteracting solutions for the A-grid. Each solution is a Z-grid solution over two grid spacings. In Figure, four independent solutions on Zgrids are indicated by different colors.

Define: 
$\phi_{i, j}(z, t) \equiv \operatorname{Re}\left\{\hat{\Phi} e^{i(k d i+\ell d j+m z-v t)}\right\} \quad$ and $\quad \phi_{i+1 / 2, j+1 / 2}(z, t) \equiv \operatorname{Re}\left\{\hat{\Phi} e^{i[k d(i+1 / 2)+\ell d(j+1 / 2)+m z-v t]}\right\}$

Using (8) in (1):

$$
-i v \hat{\omega}_{z}=-f \hat{D}
$$

Using (8) in (2):

$$
-\underset{\sim}{i} v \hat{D}=f \hat{\omega}_{z}-\left(\frac{e^{i 2 k d}+e^{-i 2 k d}+e^{i 2 \ell d}+e^{-i 2 \ell d}-4}{4 d^{2}}\right) \hat{P}
$$

Using (8) in (7):

$$
\left(-v^{2}+N^{2}\right) \hat{D}-\left[m^{2}+\left(\frac{1}{2 \rho_{0}} \frac{\partial \rho_{0}}{\partial z}\right)^{2}\right] i v P_{i, j}=0
$$

In (10):

$$
\frac{e^{i 2 k d}+e^{-i 2 k d}+e^{i 2 \ell d}+e^{-i 2 \ell d}-4}{4 d^{2}}=\frac{2 \cos (2 k d)+2 \cos (2 \ell d)-4}{4 d^{2}}=\left(\frac{-\sin ^{2}(k d)}{(k d)^{2}} k^{2}+\frac{-\sin ^{2}(\ell d)}{(\ell d)^{2}} \ell^{2}\right)
$$

Rewrite (13):

$$
\frac{e^{i 2 k d}+e^{-i 2 k d}+e^{i 2 \ell d}+e^{-i 2 \ell d}-4}{4 d^{2}}=-\tilde{\xi}^{2} k^{2}-\tilde{\eta}^{2} \ell^{2}
$$

where

$$
\tilde{\xi} \equiv \frac{\sin (k d)}{k d} \quad \text { and } \quad \tilde{\eta} \equiv \frac{\sin (\ell d)}{\ell d}
$$

Using (12) and (14a) in (9)-(11):

$$
\begin{gathered}
-i v \hat{\omega}_{z}=-f \hat{D} \\
-i v \hat{D}=\left(\tilde{\xi}^{2} k^{2}+\tilde{\eta}^{2} \ell^{2}\right) \hat{P}+f \hat{\omega}_{z}
\end{gathered}
$$




$$
\mu\left(-v^{2}+N^{2}\right) \hat{D}+\left[\frac{\partial^{2}}{\partial z^{2}}-\left(\frac{1}{2 \rho_{0}} \frac{\partial \rho_{0}}{\partial z}\right)^{2}\right] i v \hat{P}=0
$$

From (20) and (21):

$$
\left(v^{2}-f^{2}\right) \hat{D}=\left(\tilde{\xi}^{2} k^{2}+\tilde{\eta}^{2} \ell^{2}\right) i v \hat{P}
$$

From (22) and (23):

$$
v^{2}=\frac{N^{2}\left(\tilde{\xi}^{2} k^{2}+\tilde{\eta}^{2} \ell^{2}\right)+f^{2}\left(m^{2}+\frac{1}{4 H^{2}}\right)}{\left(\tilde{\xi}^{2} k^{2}+\tilde{\eta}^{2} \ell^{2}\right)+\left(m^{2}+\frac{1}{4 H^{2}}\right)}
$$

\section{Rossby modes}

Baroclinic modes:

Adding the beta effect to the vorticity equation (1):

$$
\frac{\partial\left(\omega_{z}\right)_{i, j}}{\partial t}=-f_{0} D_{i, j}-\frac{\beta}{f_{0}} \frac{P_{i+1, j}-P_{i-1, j}}{2 d}
$$

Dropping $\partial D / \partial t$ in (2):

$$
0=-\frac{P_{i+2, j}+P_{i-2, j}+P_{i, j+2}+P_{i, j-2}-4 P_{i, j}}{4 d^{2}}+f\left(\omega_{z}\right)_{i, j}
$$

Dropping $\partial^{2} D / \partial t^{2}$ in (7):

$$
N^{2} D_{i, j}-\left[\frac{\partial^{2}}{\partial z^{2}}-\left(\frac{1}{2 \rho_{0}} \frac{\partial \rho_{0}}{\partial z}\right)^{2}\right] \frac{\partial}{\partial t} P_{i, j}=0
$$

From (30)-(32): 


$$
\begin{gathered}
-\underset{\sim}{i v} \hat{\omega}_{z}=-f_{0} \hat{D}-\frac{\beta}{f_{0}} \frac{e^{i k d}-e^{-i k d}}{2 d} \hat{P} \\
0=\left(\tilde{\xi}^{2} k^{2}+\tilde{\eta}^{2} \ell^{2}\right) \hat{P}+f_{0} \hat{\omega}_{z} \\
N^{2} \hat{D}-\left[m^{2}+\left(\frac{1}{2 \rho_{0}} \frac{\partial \rho_{0}}{\partial z}\right)^{2}\right] \underset{\sim}{i} v \hat{P}=0
\end{gathered}
$$

Rewrite (40):

$$
-\underset{\sim}{i v \hat{\omega}_{z}}=-f_{0} \hat{D}-\underset{\sim}{i} \frac{\beta}{f_{0}} \frac{2 \sin (k d)}{2 d} \hat{P}
$$

Rewrite (43):

$$
-\underset{\sim}{v} v \hat{\omega}_{z}=-f_{0} \hat{D}-\underset{\sim}{i} \frac{\beta}{f_{0}} \tilde{\xi}_{k} \hat{P}
$$

where $\tilde{\xi} \equiv \frac{\sin (k d)}{k d}$ is given by $(14 \mathrm{~b})$.

From (41), (42) and (44):

$$
v N^{2}\left(\tilde{\xi}^{2} k^{2}+\tilde{\eta}^{2} \ell^{2}\right) \hat{P}=-f_{0}^{2}\left[m^{2}+\left(\frac{1}{2 \rho_{0}} \frac{\partial \rho_{0}}{\partial z}\right)^{2}\right] v \hat{P}-N^{2} \beta \tilde{\xi} k \hat{P}
$$

Using $\frac{1}{\rho_{0}} \frac{\partial \rho_{0}}{\partial z}=-\frac{1}{H}$ in (46) and for nontrivial solutions $(\hat{P} \neq 0)$ :

$$
v=\frac{-\beta \tilde{\xi}_{k}}{\left(\tilde{\xi}^{2} k^{2}+\tilde{\eta}^{2} \ell^{2}\right)+\frac{f_{0}^{2}}{N^{2}}\left(m^{2}+\frac{1}{4 H}\right)}
$$

Barotropic modes: 
Assuming $D=0$ in (30)

$$
\frac{\partial\left(\omega_{z}\right)_{i, j}}{\partial t}=-\frac{\beta}{f_{0}} \frac{P_{i+1, j}-P_{i-1, j}}{2 d}
$$

Writing (31) again:

$$
0=-\frac{P_{i+2, j}+P_{i-2, j}+P_{i, j+2}+P_{i, j-2}-4 P_{i, j}}{4 d^{2}}+f\left(\omega_{z}\right)_{i, j}
$$

Assuming $D=0$ in (32)

$$
-\left[\frac{\partial^{2}}{\partial z^{2}}-\left(\frac{1}{2 \rho_{0}} \frac{\partial \rho_{0}}{\partial z}\right)^{2}\right] \frac{\partial}{\partial t} P_{i, j}=0
$$

( $\mathrm{P}$ is vertically constant).

From (50) and (51):

$$
0=v\left(\tilde{\xi}^{2} k^{2}+\tilde{\eta}^{2} \ell^{2}\right) \hat{P}+\beta \tilde{\xi} k \hat{P}
$$

From (53) and for nontrivial solutions $(\hat{P} \neq 0)$ :

$$
v=\frac{-\beta \tilde{\xi}_{k}}{\tilde{\xi}^{2} k^{2}+\tilde{\eta}^{2} \ell^{2}}
$$




\section{E-grid}

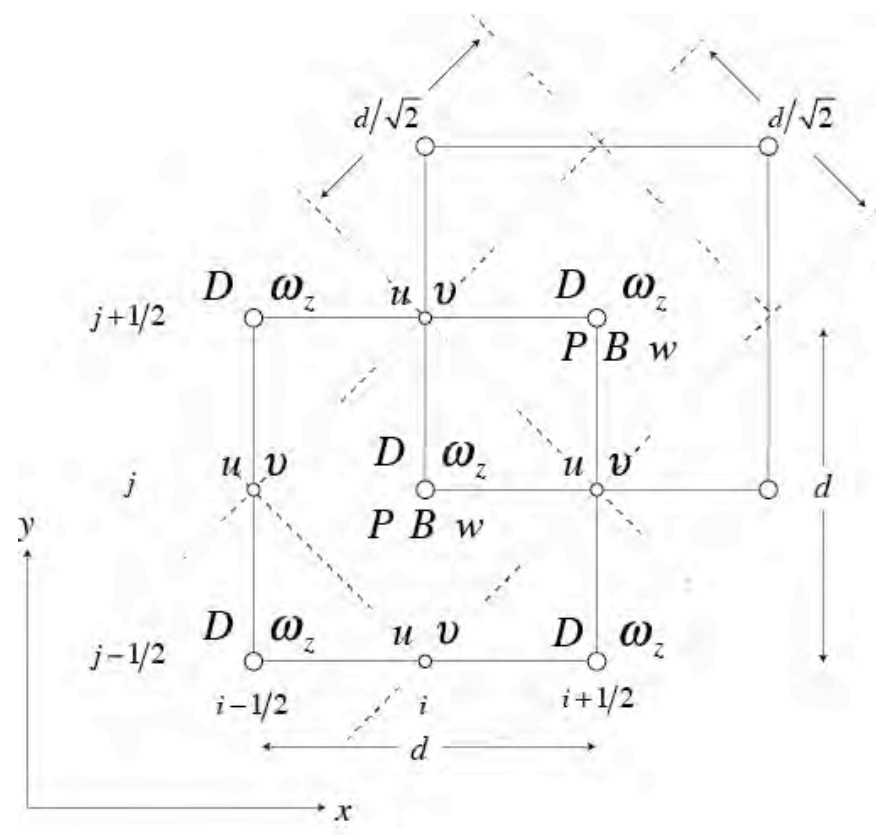

For $(\mathbf{i}, \mathrm{j})$ points (black grid points in Figure):

\section{Inertia-gravity modes}

Horizontal momentum equation:

$\frac{\partial u_{i, j+1 / 2}}{\partial t}=-\frac{P_{i+1 / 2, j+1 / 2}-P_{i-1 / 2, j+1 / 2}}{d}+f v_{i, j+1 / 2}$

$\frac{\partial u_{i+1 / 2, j}}{\partial t}=-\frac{P_{i+1, j}-P_{i, j}}{d}+f v_{i+1 / 2, j}$

$\frac{\partial v_{i, j+1 / 2}}{\partial t}=-\frac{P_{i, j+1}-P_{i, j}}{d}-f u_{i, j+1 / 2}$ 
$\frac{\partial v_{i+1 / 2, j}}{\partial t}=-\frac{P_{i+1 / 2, j+1 / 2}-P_{i+1 / 2, j-1 / 2}}{d}-f u_{i+1 / 2, j}$

To derive divergence equation for corner points $(i, j)$ :

$\begin{aligned} \frac{\partial}{\partial t}\left(\frac{u_{i, j+1 / 2}+u_{i+1 / 2, j}}{2}\right) & =-\frac{P_{i+1 / 2, j+1 / 2}-P_{i-1 / 2, j+1 / 2}}{2 d}-\frac{P_{i+1, j}-P_{i, j}}{2 d}+f\left(\frac{v_{i, j+1 / 2}+v_{i+1 / 2, j}}{2}\right) \\ \frac{\partial}{\partial t}\left(\frac{v_{i, j+1 / 2}+v_{i+1 / 2, j}}{2}\right) & =-\frac{P_{i, j+1}-P_{i, j}}{2 d}-\frac{P_{i+1 / 2, j+1 / 2}-P_{i+1 / 2, j-1 / 2}}{2 d}-f\left(\frac{u_{i, j+1 / 2}+u_{i+1 / 2, j}}{2}\right)\end{aligned}$

$\frac{\partial}{\partial t}\left(\frac{u_{i-1 / 2, j}+u_{i, j+1 / 2}}{2}\right)=-\frac{P_{i, j}-P_{i-1, j}}{2 d}-\frac{P_{i+1 / 2, j+1 / 2}-P_{i-1 / 2, j+1 / 2}}{2 d}+f\left(\frac{v_{i-1 / 2, j}+v_{i, j+1 / 2}}{2}\right)$

$\frac{\partial}{\partial t}\left(\frac{v_{i-1 / 2, j}+v_{i, j+1 / 2}}{2}\right)=-\frac{P_{i-1 / 2, j+1 / 2}-P_{i-1 / 2, j-1 / 2}}{2 d}-\frac{P_{i, j+1}-P_{i, j}}{2 d}-f\left(\frac{u_{i-1 / 2, j}+u_{i, j+1 / 2}}{2}\right)$

$\frac{\partial}{\partial t}\left(\frac{u_{i, j-1 / 2}+u_{i-1 / 2, j}}{2}\right)=-\frac{P_{i+1 / 2, j-1 / 2}-P_{i-1 / 2, j-1 / 2}}{2 d}-\frac{P_{i, j}-P_{i-1, j}}{2 d}+f\left(\frac{v_{i, j-1 / 2}+v_{i-1 / 2, j}}{2}\right)$

$\frac{\partial}{\partial t}\left(\frac{v_{i, j-1 / 2}+v_{i-1 / 2, j}}{2}\right)=-\frac{P_{i, j}-P_{i, j-1}}{2 d}-\frac{P_{i-1 / 2, j+1 / 2}-P_{i-1 / 2, j-1 / 2}}{2 d}-f\left(\frac{u_{i, j-1 / 2}+u_{i-1 / 2, j}}{2}\right)$

$\frac{\partial}{\partial t}\left(\frac{u_{i+1 / 2, j}+u_{i, j-1 / 2}}{2}\right)=-\frac{P_{i+1, j}-P_{i, j}}{2 d}-\frac{P_{i+1 / 2, j-1 / 2}-P_{i-1 / 2, j-1 / 2}}{2 d}+f\left(\frac{v_{i+1 / 2, j}+v_{i, j-1 / 2}}{2}\right)$

$\frac{\partial}{\partial t}\left(\frac{v_{i+1 / 2, j}+v_{i, j-1 / 2}}{2}\right)=-\frac{P_{i+1 / 2, j+1 / 2}-P_{i+1 / 2, j-1 / 2}}{2 d}-\frac{P_{i, j}-P_{i, j-1}}{2 d}-f\left(\frac{u_{i+1 / 2, j}+u_{i, j-1 / 2}}{2}\right)$

Define divergence at $(i, j)$, [walls of the cell $(i, j)$ is marked by the dashed lines, the cell area is $d^{2} / 2$, cell wall length is $d / \sqrt{2}$ ]:

$$
\begin{aligned}
D_{i, j} & =\alpha \frac{d}{\sqrt{2}}\left(\frac{u_{i, j+1 / 2}+u_{i+1 / 2, j}}{2 d^{2} / 2}+\frac{v_{i, j+1 / 2}+v_{i+1 / 2, j}}{2 d^{2} / 2}\right)+\alpha \frac{d}{\sqrt{2}}\left(-\frac{u_{i-1 / 2, j}+u_{i, j+1 / 2}}{2 d^{2} / 2}+\frac{v_{i-1 / 2, j}+v_{i, j+1 / 2}}{2 d^{2} / 2}\right) \\
& +\alpha \frac{d}{\sqrt{2}}\left(-\frac{u_{i, j-1 / 2}+u_{i-1 / 2, j}}{2 d^{2} / 2}-\frac{v_{i, j-1 / 2}+v_{i-1 / 2, j}}{2 d^{2} / 2}\right)+\alpha \frac{d}{\sqrt{2}}\left(\frac{u_{i+1 / 2, j}+u_{i, j-1 / 2}}{2 d^{2} / 2}-\frac{v_{i+1 / 2, j}+v_{i, j-1 / 2}}{2 d^{2} / 2}\right)
\end{aligned}
$$


where

$$
\alpha \equiv \cos \left(45^{\circ}\right)=\sin \left(45^{\circ}\right)=\frac{\sqrt{2}}{2}
$$

[The normal component of velocity to cell walls (dashed lines) uses alpha] Simplifying (M.8):

$$
D_{i, j}=\frac{1}{d}\left(u_{i+1 / 2, j}+v_{i, j+1 / 2}-u_{i-1 / 2, j}-v_{i, j-1 / 2}\right)
$$

Divergence equation (1):

Using $\partial / \partial t$ of (M.10) then using (M.1.a), (M.1.b), (M.2.a) and (M.2.b):

$$
\frac{\partial}{\partial t} D_{i, j}=-\frac{1}{d^{2}}\left(P_{i+1, j}+P_{i-1, j}+P_{i, j+1}+P_{i, j-1}-4 P_{i, j}\right)+f \frac{1}{d}\left(v_{i+1 / 2, j}-u_{i, j+1 / 2}-v_{i-1 / 2, j}+u_{i, j-1 / 2}\right)
$$

Define:

$$
\left(\omega_{z}\right)_{i, j} \equiv \frac{1}{d}\left(v_{i+1 / 2, j}-u_{i, j+1 / 2}-v_{i-1 / 2, j}+u_{i, j-1 / 2}\right)
$$

Divergence equation (2):

Consider (M.6) and (M.7):

$$
\frac{\partial}{\partial t} D_{i, j}=-\frac{1}{d^{2}}\left(P_{i+1, j}+P_{i-1, j}+P_{i, j+1}+P_{i, j-1}-4 P_{i, j}\right)+f\left(\omega_{z}\right)_{i, j}
$$

To derive vorticity equation for center point $(i, j)$. take $\partial / \partial t$ (M.7) and use (M.1.a)-(M.2.b): 


$$
\begin{gathered}
\frac{\partial}{\partial t}\left(\omega_{z}\right)_{i, j} \equiv \frac{1}{d}\left(-\frac{P_{i+1 / 2, j+1 / 2}-P_{i+1 / 2, j-1 / 2}}{d}+\frac{P_{i+1 / 2, j+1 / 2}-P_{j-1 / 2, j+1 / 2}}{d}+\frac{P_{i-1 / 2, j+1 / 2}-P_{i-1 / 2, j-1 / 2}}{d}-\frac{P_{i+1 / 2, j-1 / 2}-P_{i-1 / 2, j-1 / 2}}{d}\right) \\
-\frac{f}{d}\left(u_{i+1 / 2, j}+v_{i, j+1 / 2}-u_{i-1 / 2, j}-v_{i, j-1 / 2}\right)
\end{gathered}
$$

The vorticity equation:

Using (M.10) in (M.9):

$$
\frac{\partial}{\partial t}\left(\omega_{z}\right)_{i, j}=-f D_{i, j}
$$

Vorticity equation from (M.12):

$$
\frac{\partial}{\partial t}\left(\omega_{z}\right)_{i, j}=-f D_{i, j}
$$

Divergence equation from (M.8):

$$
\frac{\partial}{\partial t} D_{i, j}=-\frac{1}{d^{2}}\left(P_{i+1, j}+P_{i-1, j}+P_{i, j+1}+P_{i, j-1}-4 P_{i, j}\right)+f\left(\omega_{z}\right)_{i, j}
$$

From (1) and (2), we obtain

$$
\left(\frac{\partial^{2}}{\partial t^{2}}+f^{2}\right) D_{i, j}=-\frac{\partial}{\partial t}\left[\frac{1}{d^{2}}\left(P_{i+1, j}+P_{i-1, j}+P_{i, j+1}+P_{i, j-1}-4 P_{i, j}\right)\right]
$$

Vertical momentum equation:

$$
\frac{\partial w_{i, j}}{\partial t}=-\left[\frac{\partial}{\partial z}-\left(\frac{1}{2 \rho_{0}} \frac{\partial \rho_{0}}{\partial z}\right)\right] P_{i, j}+B_{i, j}
$$

Thermodynamic equation: 


$$
\frac{\partial B_{i, j}}{\partial t}=-N^{2} w_{i, j}
$$

Mass continuity equation:

$$
D_{i, j}+\left[\frac{\partial}{\partial z}+\left(\frac{1}{2 \rho_{0}} \frac{\partial \rho_{0}}{\partial z}\right)\right] w_{i, j}=0
$$

From (4)-(6):

$$
\left(\frac{\partial^{2}}{\partial t^{2}}+N^{2}\right) D_{i, j}-\left[\frac{\partial^{2}}{\partial z^{2}}-\left(\frac{1}{2 \rho_{0}} \frac{\partial \rho_{0}}{\partial z}\right)^{2}\right] \frac{\partial}{\partial t} P_{i, j}=0
$$

From (2') and (7), we can write the following two equations:

$$
\left[\frac{\partial^{2}}{\partial z^{2}}-\left(\frac{1}{2 \rho_{0}} \frac{\partial \rho_{0}}{\partial z}\right)^{2}\right]\left(\frac{\partial^{2}}{\partial t^{2}}+f^{2}\right) D_{i, j}=-\left(\frac{\partial^{2}}{\partial t^{2}}+N^{2}\right)\left[\frac{1}{d^{2}}\left(D_{i+1, j}+D_{i-1, j}+D_{i, j+1}+D_{i, j-1}-4 D_{i, j}\right)\right]
$$

and

$$
\left(\frac{\partial^{2}}{\partial t^{2}}+f^{2}\right)\left[\frac{\partial^{2}}{\partial z^{2}}-\left(\frac{1}{2 \rho_{0}} \frac{\partial \rho_{0}}{\partial z}\right)^{2}\right] \frac{\partial}{\partial t} P_{i, j}=-\left(\frac{\partial^{2}}{\partial t^{2}}+N^{2}\right) \frac{\partial}{\partial t}\left[\frac{1}{d^{2}}\left(P_{i+1, j}+P_{i-1, j}+P_{i, j+1}+P_{i, j-1}-4 P_{i, j}\right)\right]
$$

Horizontal differencing does not use information from half-integer grid points. The solution is independent from the solution on the half-integer grid points.

Define:

$\phi_{i, j}(z, t) \equiv \operatorname{Re}\left\{\hat{\Phi} e^{i(k d i+\ell d j+m z-v t)}\right\} \quad$ and $\quad \phi_{i+1 / 2, j+1 / 2}(z, t) \equiv \operatorname{Re}\left\{\hat{\Phi} e^{i[k d(i+1 / 2)+\ell d(j+1 / 2)+m z-v t]}\right\}$

Using (8) in (1):

$$
-i v \hat{\omega}_{z}=-f \hat{D}
$$


Using (8) in (2):

$$
-\underset{\sim}{i v} \hat{D}=f \hat{\omega}_{z}-\left(\frac{e^{i k d}+e^{-i k d}+e^{i \ell d}+e^{-i \ell d}-4}{d^{2}}\right) \hat{P}
$$

Using (8) in (7):

$$
\left(-v^{2}+N^{2}\right) \hat{D}-\left[m^{2}+\left(\frac{1}{2 \rho_{0}} \frac{\partial \rho_{0}}{\partial z}\right)^{2}\right] i v P_{i, j}=0
$$

In (10):

$$
\begin{aligned}
\frac{e^{i k d}+e^{-i k d}+e^{i \ell l d}+e^{-i \ell l d}-4}{d^{2}} & =\frac{2 \cos (k d)+2 \cos (\ell d)-4}{d^{2}} \\
& =\left(\frac{-\sin ^{2}\left(\frac{1}{2} k d\right)}{\left(\frac{1}{2} k d\right)^{2}} k^{2}+\frac{-\sin ^{2}\left(\frac{1}{2} \ell d\right)}{\left(\frac{1}{2} \ell d\right)^{2}} \ell^{2}\right)
\end{aligned}
$$

Rewrite (13):

$$
\frac{e^{i k d}+e^{-i k d}+e^{i \ell d}+e^{-i \ell d}-4}{d^{2}}=-\xi^{2} k^{2}-\eta^{2} \ell^{2}
$$

where

$$
\xi \equiv \frac{\sin \left(\frac{1}{2} k d\right)}{\frac{1}{2} k d} \quad \text { and } \quad \eta \equiv \frac{\sin \left(\frac{1}{2} \ell d\right)}{\frac{1}{2} \ell d}
$$

Using (12) and (14a) in (9)-(11):

$$
\begin{gathered}
-i v \hat{\omega}_{z}=-f \hat{D} \\
-i v \hat{D}=\left(\xi^{2} k^{2}+\eta^{2} \ell^{2}\right) \hat{P}+f \hat{\omega}_{z} \\
\left(-v^{2}+N^{2}\right) \hat{D}+\left[\frac{\partial^{2}}{\partial z^{2}}-\left(\frac{1}{2 \rho_{0}} \frac{\partial \rho_{0}}{\partial z}\right)^{2}\right] i v \hat{P}=0
\end{gathered}
$$


From (20) and (21):

$$
\left(v^{2}-f^{2}\right) \hat{D}=\left(\xi^{2} k^{2}+\eta^{2} \ell^{2}\right) i v \hat{P}
$$

From (22) and (23):

$$
v^{2}=\frac{N^{2}\left(\xi^{2} k^{2}+\eta^{2} \ell^{2}\right)+f^{2}\left(m^{2}+\frac{1}{4 H^{2}}\right)}{\left(\xi^{2} k^{2}+\eta^{2} \ell^{2}\right)+\left(m^{2}+\frac{1}{4 H^{2}}\right)}
$$

\section{Rossby modes}

Baroclinic modes:

Adding the beta effect to the vorticity equation (1):

$$
\frac{\partial\left(\omega_{z}\right)_{i, j}}{\partial t}=-f_{0} D_{i, j}-\frac{\beta}{f_{0}} \frac{P_{i+1, j}-P_{i-1, j}}{2 d}
$$

Dropping $\partial D / \partial t$ in (2):

$$
0=-\frac{1}{d^{2}}\left(P_{i+1, j}+P_{i-1, j}+P_{i, j+1}+P_{i, j-1}-4 P_{i, j}\right)+f\left(\omega_{z}\right)_{i, j}
$$

Dropping $\partial^{2} D / \partial t^{2}$ in (7):

$$
N^{2} D_{i, j}-\left[\frac{\partial^{2}}{\partial z^{2}}-\left(\frac{1}{2 \rho_{0}} \frac{\partial \rho_{0}}{\partial z}\right)^{2}\right] \frac{\partial}{\partial t} P_{i, j}=0
$$

From (30)-(32):

$$
-\underset{\sim}{i} v \hat{\omega}_{z}=-f_{0} \hat{D}-\frac{\beta}{f_{0}} \frac{e^{i k d}-e^{-i k d}}{2 d} \hat{P}
$$




$$
\begin{gathered}
0=\left(\xi^{2} k^{2}+\eta^{2} \ell^{2}\right) \hat{P}+f_{0} \hat{\omega}_{z} \\
N^{2} \hat{D}-\left[m^{2}+\left(\frac{1}{2 \rho_{0}} \frac{\partial \rho_{0}}{\partial z}\right)^{2}\right] \underset{\sim}{i} \nu \hat{P}=0
\end{gathered}
$$

Rewrite (40):

$$
-\underset{\sim}{i} v \hat{\omega}_{z}=-f_{0} \hat{D}-\underset{\sim}{i} \frac{\beta}{f_{0}} \frac{2 \sin (k d)}{2 d} \hat{P}
$$

Rewrite (43):

$$
-\underset{\sim}{v} v \hat{\omega}_{z}=-f_{0} \hat{D}-\underset{\sim}{i} \frac{\beta}{f_{0}} \tilde{\xi}_{k} \hat{P}
$$

where

$$
\tilde{\xi} \equiv \frac{\sin (k d)}{k d}
$$

From (41), (42) and (44):

$$
v N^{2}\left(\xi^{2} k^{2}+\eta^{2} \ell^{2}\right) \hat{P}=-f_{0}^{2}\left[m^{2}+\left(\frac{1}{2 \rho_{0}} \frac{\partial \rho_{0}}{\partial z}\right)^{2}\right] v \hat{P}-N^{2} \beta \tilde{\xi}_{k} \hat{P}
$$

Using $\frac{1}{\rho_{0}} \frac{\partial \rho_{0}}{\partial z}=-\frac{1}{H}$ in (46) and for nontrivial solutions $(\hat{P} \neq 0)$ :

$$
v=\frac{-\beta \tilde{\xi}_{k}}{\left(\xi^{2} k^{2}+\eta^{2} \ell^{2}\right)+\frac{f_{0}^{2}}{N^{2}}\left(m^{2}+\frac{1}{4 H}\right)}
$$

Barotropic modes:

Assuming $D=0$ in (30)

$$
\frac{\partial\left(\omega_{z}\right)_{i, j}}{\partial t}=-\frac{\beta}{f_{0}} \frac{P_{i+1, j}-P_{i-1, j}}{2 d}
$$


Writing (31) again:

$$
0=-\frac{1}{d^{2}}\left(P_{i+1, j}+P_{i-1, j}+P_{i, j+1}+P_{i, j-1}-4 P_{i, j}\right)+f\left(\omega_{z}\right)_{i, j}
$$

Assuming $D=0$ in (32)

$$
-\left[\frac{\partial^{2}}{\partial z^{2}}-\left(\frac{1}{2 \rho_{0}} \frac{\partial \rho_{0}}{\partial z}\right)^{2}\right] \frac{\partial}{\partial t} P_{i, j}=0
$$

( $P$ is vertically constant).

From (50) and (51):

$$
0=v\left(\xi^{2} k^{2}+\eta^{2} \ell^{2}\right) \hat{P}+\beta \tilde{\xi}_{k} \hat{P}
$$

From (53) and for nontrivial solutions $(\hat{P} \neq 0)$ :

$$
v=\frac{-\beta \tilde{\xi}_{k}}{\xi^{2} k^{2}+\eta^{2} \ell^{2}}
$$

\section{For $(i+1 / 2, j+1 / 2)$ points (red grid points in Figure):}

Following derivations parallel to $\left(7^{\prime}\right)$ and $\left(7^{\prime \prime}\right)$, we obtain

$$
\begin{aligned}
& {\left[\frac{\partial^{2}}{\partial z^{2}}-\left(\frac{1}{2 \rho_{0}} \frac{\partial \rho_{0}}{\partial z}\right)^{2}\right]\left(\frac{\partial^{2}}{\partial t^{2}}+f^{2}\right) D_{i+1 / 2, j+1 / 2}=} \\
& \quad-\left(\frac{\partial^{2}}{\partial t^{2}}+N^{2}\right)\left[\frac{1}{d^{2}}\left(D_{i+3 / 2, j+1 / 2}+D_{i-3 / 2, j+1 / 2}+D_{i+1 / 2, j+3 / 2}+D_{i+1 / 2, j-3 / 2}-4 D_{i+1 / 2, j+1 / 2}\right)\right]
\end{aligned}
$$

and 


$$
\begin{aligned}
\left(\frac{\partial^{2}}{\partial t^{2}}+f^{2}\right) & {\left[\frac{\partial^{2}}{\partial z^{2}}-\left(\frac{1}{2 \rho_{0}} \frac{\partial \rho_{0}}{\partial z}\right)^{2}\right] \frac{\partial}{\partial t} P_{i+1 / 2, j+1 / 2}=} \\
& -\left(\frac{\partial^{2}}{\partial t^{2}}+N^{2}\right) \frac{\partial}{\partial t}\left[\frac{1}{d^{2}}\left(P_{i+3 / 2, j+1 / 2}+P_{i-3 / 2, j+1 / 2}+P_{i+1 / 2, j+3 / 2}+P_{i+1 / 2, j-3 / 2}-4 P_{i+1 / 2, j+1 / 2}\right)\right]
\end{aligned}
$$

Horizontal differencing does not use information from the integer grid points. The solution is independent from the solution on the integer grid points.

\section{Inertia-gravity modes}

$$
v^{2}=\frac{N^{2}\left(\xi^{2} k^{2}+\eta^{2} \ell^{2}\right)+f^{2}\left(m^{2}+\frac{1}{4 H^{2}}\right)}{\left(\xi^{2} k^{2}+\eta^{2} \ell^{2}\right)+\left(m^{2}+\frac{1}{4 H^{2}}\right)}
$$

\section{Rossby modes}

Baroclinic modes:

$$
v=\frac{-\beta \tilde{\xi}_{k}}{\left(\xi^{2} k^{2}+\eta^{2} \ell^{2}\right)+\frac{f_{0}^{2}}{N^{2}}\left(m^{2}+\frac{1}{4 H}\right)}
$$

Barotropic modes:

$$
v=\frac{-\beta \tilde{\xi}_{k}}{\xi^{2} k^{2}+\eta^{2} \ell^{2}}
$$




\section{B-grid}
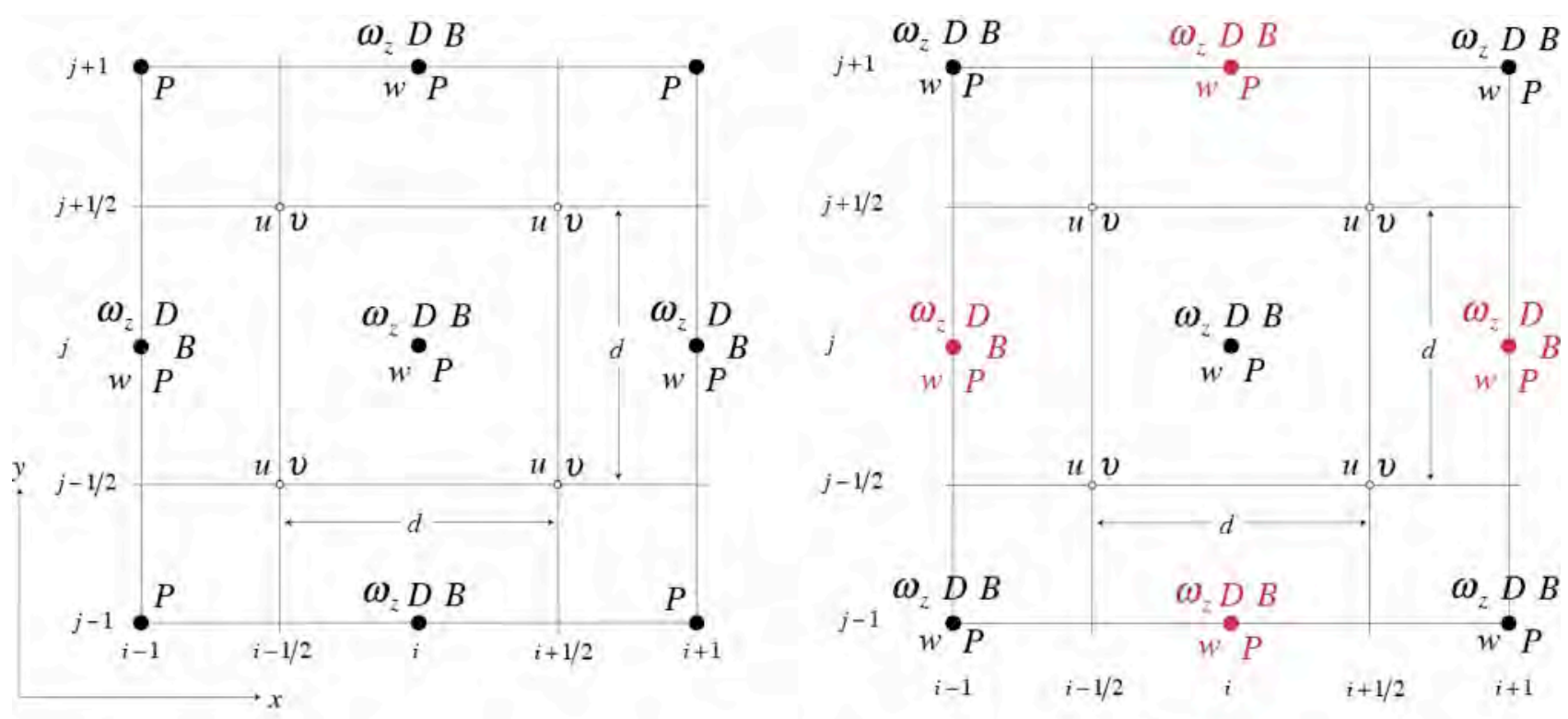

B-grid produces two independent and noninteracting solutions.

Figure. B grid

\section{Inertia-gravity modes}

Horizontal momentum equation:

$$
\begin{aligned}
& \frac{\partial u_{i+1 / 2, j+1 / 2}}{\partial t}=-\frac{\frac{1}{2}\left(P_{i+1, j+1}+P_{i+1, j}\right)-\frac{1}{2}\left(P_{i, j+1}+P_{i, j}\right)}{d}+f v_{i+1 / 2, j+1 / 2} \\
& \frac{\partial v_{i+1 / 2, j+1 / 2}}{\partial t}=-\frac{\frac{1}{2}\left(P_{i+1, j+1}+P_{i, j+1}\right)-\frac{1}{2}\left(P_{i+1, j}+P_{i, j}\right)}{d}-f u_{i+1 / 2, j+1 / 2}
\end{aligned}
$$

To derive divergence equation for center points $(i, j)$ : 


$$
\begin{aligned}
& \frac{\partial}{\partial t}\left(\frac{u_{i+1 / 2, j+1 / 2}+u_{i+1 / 2, j-1 / 2}}{2 d}-\frac{u_{i-1 / 2, j+1 / 2}+u_{i-1 / 2, j-1 / 2}}{2 d}\right)= \\
& -\frac{\frac{1}{2}\left(P_{i+1, j+1}+P_{i+1, j}\right)-\frac{1}{2}\left(P_{i, j+1}+P_{i, j}\right)}{2 d}-\frac{\frac{1}{2}\left(P_{i+1, j}+P_{i+1, j-1}\right)-\frac{1}{2}\left(P_{i, j}+P_{i, j-1}\right)}{2 d} \\
& +\frac{\frac{1}{2}\left(P_{i, j+1}+P_{i, j}\right)-\frac{1}{2}\left(P_{i-1, j+1}+P_{i-1, j}\right)}{2 d}+\frac{\frac{1}{2}\left(P_{i, j}+P_{i, j-1}\right)-\frac{1}{2}\left(P_{i-1, j}+P_{i-1, j-1}\right)}{2 d} \\
& +f\left(\frac{v_{i+1 / 2, j+1 / 2}+v_{i+1 / 2, j-1 / 2}}{2 d}-\frac{v_{i-1 / 2, j+1 / 2}+v_{i-1 / 2, j-1 / 2}}{2 d}\right)
\end{aligned}
$$

$$
\begin{aligned}
\frac{\partial}{\partial t}\left(\frac{v_{i+1 / 2, j+1 / 2}+v_{i-1 / 2, j+1 / 2}}{2 d}-\frac{\left.v_{i+1 / 2, j-1 / 2}+v_{i-1 / 2, j-1 / 2}\right)}{2 d}\right)= & \\
& -\frac{\frac{1}{2}\left(P_{i+1, j+1}+P_{i, j+1}\right)-\frac{1}{2}\left(P_{i+1, j}+P_{i, j}\right)}{2 d}-\frac{\frac{1}{2}\left(P_{i, j+1}+P_{i-1, j+1}\right)-\frac{1}{2}\left(P_{i, j}+P_{i-1, j}\right)}{2 d} \\
& +\frac{\frac{1}{2}\left(P_{i+1, j}+P_{i, j}\right)-\frac{1}{2}\left(P_{i+1, j-1}+P_{i, j-1}\right)}{2 d}+\frac{\frac{1}{2}\left(P_{i, j}+P_{i-1, j}\right)-\frac{1}{2}\left(P_{i, j-1}+P_{i-1, j-1}\right)}{2 d} \\
& -f\left(\frac{u_{i+1 / 2, j+1 / 2}+u_{i-1 / 2, j+1 / 2}}{2 d}-\frac{u_{i+1 / 2, j-1 / 2}+u_{i-1 / 2, j-1 / 2}}{2 d}\right)
\end{aligned}
$$

Define:

$$
D_{i+1 / 2, j+1 / 2} \equiv \frac{1}{d}\left(\frac{u_{i+1 / 2, j+1 / 2}+u_{i+1 / 2, j-1 / 2}}{2}-\frac{u_{i-1 / 2, j+1 / 2}+u_{i-1 / 2, j-1 / 2}}{2}+\frac{v_{i+1 / 2, j+1 / 2}+v_{i-1 / 2, j+1 / 2}}{2}-\frac{v_{i+1 / 2, j-1 / 2}+v_{i-1 / 2, j-1 / 2}}{2}\right)
$$

Divergence equation (1):

$$
\begin{aligned}
\frac{\partial}{\partial t} D_{i, j} & =-\frac{P_{i+1, j+1}+P_{i-1, j+1}+P_{i+1, j-1}+P_{i-1, j-1}-4 P_{i, j}}{2 d^{2}} \\
& +f\left(\frac{v_{i+1 / 2, j+1 / 2}+v_{i+1 / 2, j-1 / 2}}{2 d}-\frac{u_{i+1 / 2, j+1 / 2}+u_{i-1 / 2, j+1 / 2}}{2 d}-\frac{v_{i-1 / 2, j+1 / 2}+v_{i-1 / 2, j-1 / 2}}{2 d}+\frac{u_{i+1 / 2, j-1 / 2}+u_{i-1 / 2, j-1 / 2}}{2 d}\right)
\end{aligned}
$$

\section{Define:}




$$
\left(\omega_{z}\right)_{i, j} \equiv\left(\frac{v_{i+1 / 2, j+1 / 2}+v_{i+1 / 2, j-1 / 2}}{2 d}-\frac{u_{i+1 / 2, j+1 / 2}+u_{i-1 / 2, j+1 / 2}}{2 d}-\frac{v_{i-1 / 2, j+1 / 2}+v_{i-1 / 2, j-1 / 2}}{2 d}+\frac{u_{i+1 / 2, j-1 / 2}+u_{i-1 / 2, j-1 / 2}}{2 d}\right)
$$

Divergence equation (2):

Consider (M.6) and (M.7):

$$
\frac{\partial}{\partial t} D_{i, j}=-\frac{P_{i+1, j+1}+P_{i-1, j+1}+P_{i+1, j-1}+P_{i-1, j-1}-4 P_{i, j}}{2 d^{2}}+f\left(\omega_{z}\right)_{i, j}
$$

To derive vorticity equation for center point $(i, j)$ :

$$
\begin{array}{r}
\frac{\partial}{\partial t}\left(\frac{v_{i+1 / 2, j+1 / 2}+v_{i+1 / 2, j-1 / 2}}{2}-\frac{v_{i-1 / 2, j+1 / 2}+v_{i-1 / 2, j-1 / 2}}{2}\right) \\
2 \\
-f\left(\frac{u_{i+1 / 2, j+1 / 2}+u_{i+1 / 2, j-1 / 2}}{2}-\frac{u_{i-1 / 2, j+1 / 2}+u_{i-1 / 2, j-1 / 2}}{2}\right) \\
\frac{\partial}{\partial t}\left(-\frac{u_{i+1 / 2, j+1 / 2}+u_{i-1 / 2, j+1 / 2}}{2 d}+\frac{u_{i+1 / 2, j+1 / 2}+u_{i-1 / 2, j-1 / 2}}{2 d}\right)=\frac{1}{4 d}\left(P_{i+1, j+1}-P_{i-1, j+1}-P_{i+1, j-1}+P_{i-1, j-1}\right) \\
-f\left(\frac{v_{i+1 / 2, j+1 / 2}+v_{i-1 / 2, j+1 / 2}}{2}-\frac{v_{i+1 / 2, j-1 / 2}+v_{i-1 / 2, j-1 / 2}}{2}\right)
\end{array}
$$

The vorticity equation (1):

$$
\begin{gathered}
\frac{\partial}{\partial t}\left(\frac{v_{i+1 / 2, j+1 / 2}+v_{i+1 / 2, j-1 / 2}}{2 d}-\frac{v_{i-1 / 2, j+1 / 2}+v_{i-1 / 2, j-1 / 2}}{2 d}-\frac{u_{i+1 / 2, j+1 / 2}+u_{i-1 / 2, j+1 / 2}}{2 d}+\frac{u_{i+1 / 2, j+1 / 2}+u_{i-1 / 2, j-1 / 2}}{2 d}\right)= \\
-f\left(\frac{u_{i+1 / 2, j+1 / 2}+u_{i+1 / 2, j-1 / 2}}{2 d}-\frac{u_{i-1 / 2, j+1 / 2}+u_{i-1 / 2, j-1 / 2}}{2 d}+\frac{v_{i+1 / 2, j+1 / 2}+v_{i-1 / 2, j+1 / 2}}{2 d}-\frac{v_{i+1 / 2, j-1 / 2}+v_{i-1 / 2, j-1 / 2}}{2 d}\right)
\end{gathered}
$$

The vorticity equation (2):

Using (M.7) and (M.5) in (M.11):

$$
\frac{\partial}{\partial t}\left(\omega_{z}\right)_{i, j}=-f \frac{1}{4}\left(D_{i+1 / 2, j+1 / 2}+D_{i-1 / 2, j+1 / 2}+D_{i+1 / 2, j-1 / 2}+D_{i-1 / 2, j-1 / 2}\right)
$$


Vorticity equation from (M.12):

$$
\frac{\partial\left(\omega_{z}\right)_{i, j}}{\partial t}=-f D_{i, j}
$$

Divergence equation from (M.8):

$$
\frac{\partial}{\partial t} D_{i, j}=f\left(\omega_{z}\right)_{i, j}-\frac{P_{i+1, j+1}+P_{i-1, j+1}+P_{i+1, j-1}+P_{i-1, j-1}-4 P_{i, j}}{2 d^{2}}
$$

Using (1) in (2), we obtain

$$
\left(\frac{\partial^{2}}{\partial t^{2}}+f^{2}\right) D_{i, j}=-\frac{\partial}{\partial t} \frac{P_{i+1, j+1}+P_{i-1, j+1}+P_{i+1, j-1}+P_{i-1, j-1}-4 P_{i, j}}{2 d^{2}}
$$

Vertical momentum equation:

$$
\frac{\partial w_{i, j}}{\partial t}=-\left[\frac{\partial}{\partial z}-\left(\frac{1}{2 \rho_{0}} \frac{\partial \rho_{0}}{\partial z}\right)\right] P_{i, j}+B_{i, j}
$$

Thermodynamic equation:

$$
\frac{\partial B_{i, j}}{\partial t}=-N^{2} w_{i, j}
$$

Mass continuity equation:

$$
D_{i, j}+\left[\frac{\partial}{\partial z}+\left(\frac{1}{2 \rho_{0}} \frac{\partial \rho_{0}}{\partial z}\right)\right] w_{i, j}=0
$$


From (4)-(6):

$$
\left(\frac{\partial^{2}}{\partial t^{2}}+N^{2}\right) D_{i, j}-\left[\frac{\partial^{2}}{\partial z^{2}}-\left(\frac{1}{2 \rho_{0}} \frac{\partial \rho_{0}}{\partial z}\right)^{2}\right] \frac{\partial}{\partial t} P_{i, j}=0
$$

Using (2') in (7) we obtain the following two equations:

$$
\left[\frac{\partial^{2}}{\partial z^{2}}-\left(\frac{1}{2 \rho_{0}} \frac{\partial \rho_{0}}{\partial z}\right)^{2}\right]\left(\frac{\partial^{2}}{\partial t^{2}}+f^{2}\right) D_{i, j}=-\left(\frac{\partial^{2}}{\partial t^{2}}+N^{2}\right) \frac{D_{i+1, j+1}+D_{i-1, j+1}+D_{i+1, j-1}+D_{i-1, j-1}-4 D_{i, j}}{2 d^{2}}
$$

and

$$
\left(\frac{\partial^{2}}{\partial t^{2}}+f^{2}\right)\left[\frac{\partial^{2}}{\partial z^{2}}-\left(\frac{1}{2 \rho_{0}} \frac{\partial \rho_{0}}{\partial z}\right)^{2}\right] \frac{\partial}{\partial t} P_{i, j}=-\left(\frac{\partial^{2}}{\partial t^{2}}+N^{2}\right) \frac{\partial}{\partial t} \frac{P_{i+1, j+1}+P_{i-1, j+1}+P_{i+1, j-1}+P_{i-1, j-1}-4 P_{i, j}}{2 d^{2}}
$$

This shows that there are two independent and noninteracting solutions for the B-grid. In Figure, two independent solutions are indicated by different colors.

Define:

$\phi_{i, j}(z, t) \equiv \operatorname{Re}\left\{\hat{\Phi} e^{i(k d i+\ell d j+m z-v t)}\right\} \quad$ and $\quad \phi_{i+1 / 2, j+1 / 2}(z, t) \equiv \operatorname{Re}\left\{\hat{\Phi} e^{i[k d(i+1 / 2)+\ell d(j+1 / 2)+m z-v t]}\right\}$

Using (8) in (1):

$$
-i v \hat{\omega}_{z}=-f \hat{D}
$$

Using (8) in (2):

$$
-\underset{\sim}{v} \hat{D}=f \hat{\omega}_{z}-\left(\frac{e^{i k d} e^{i \ell d}+e^{i k d} e^{-i \ell d}+e^{i \ell d} e^{-i k d}+e^{-i \ell d} e^{-i k d}-4}{2 d^{2}}\right) \hat{P}
$$

Using (8) in (7): 


$$
\left(-v^{2}+N^{2}\right) \hat{D}-\left[m^{2}+\left(\frac{1}{2 \rho_{0}} \frac{\partial \rho_{0}}{\partial z}\right)^{2}\right] i v P_{i, j}=0
$$

$\ln (10)$ :

$$
\begin{gathered}
\frac{\left(e^{i k d}+e^{-i k d}\right)\left(e^{i \ell d}+e^{-i \ell d}\right)-4}{2 d^{2}}=\frac{4 \cos (k d) \cos (\ell d)-4}{2 d^{2}}=\frac{4\left[1-2 \sin ^{2}\left(\frac{1}{2} k d\right)\right]\left[1-2 \sin ^{2}\left(\frac{1}{2} \ell d\right)\right]-4}{2 d^{2}} \\
=-\frac{\sin ^{2}\left(\frac{1}{2} \ell d\right)}{\left(\frac{1}{2} \ell d\right)^{2}} \ell^{2}-\frac{\sin ^{2}\left(\frac{1}{2} k d\right)}{\left(\frac{1}{2} k d\right)^{2}} k^{2}+\frac{1}{2} d^{2} \frac{\sin ^{2}\left(\frac{1}{2} k d\right)}{\left(\frac{1}{2} k d\right)^{2}} k^{2} \frac{\sin ^{2}\left(\frac{1}{2} \ell d\right)}{\left(\frac{1}{2} \ell d\right)^{2}} \ell^{2}
\end{gathered}
$$

Rewrite (13):

$$
\frac{\left(e^{i k d}+e^{-i k d}\right)\left(e^{i \ell d}+e^{-i \ell d}\right)-4}{2 d^{2}}=-\xi^{2} k^{2}-\eta^{2} \ell^{2}+\frac{1}{2} d^{2} \xi^{2} k^{2} \eta^{2} \ell^{2}
$$

where

$$
\xi \equiv \frac{\sin \left(\frac{1}{2} k d\right)}{\frac{1}{2} k d} \quad \text { and } \quad \eta \equiv \frac{\sin \left(\frac{1}{2} \ell d\right)}{\frac{1}{2} \ell d}
$$

Using (14a) in (10) and repeating (9) and (11):

$$
\begin{gathered}
-i v \hat{\omega}_{z}=-f \hat{D} \\
-i v \hat{D}=\left(\xi^{2} k^{2}+\eta^{2} \ell^{2}-\frac{1}{2} d^{2} \xi^{2} k^{2} \eta^{2} \ell^{2}\right) \hat{P}+f \hat{\omega}_{z} \\
\left(-v^{2}+N^{2}\right) \hat{D}+\left[\frac{\partial^{2}}{\partial z^{2}}-\left(\frac{1}{2 \rho_{0}} \frac{\partial \rho_{0}}{\partial z}\right)^{2}\right] i v \hat{P}=0
\end{gathered}
$$

From (20) and (21):

$$
\left(v^{2}-f^{2}\right) \hat{D}=\left(\xi^{2} k^{2}+\eta^{2} \ell^{2}-\frac{1}{2} d^{2} \xi^{2} k^{2} \eta^{2} \ell^{2}\right) i v \hat{P}
$$


From (22) and (23):

$$
v^{2}=\frac{N^{2}\left(\xi^{2} k^{2}+\eta^{2} \ell^{2}-\frac{1}{2} d^{2} \xi^{2} k^{2} \eta^{2} \ell^{2}\right)+f^{2}\left(m^{2}+\frac{1}{4 H^{2}}\right)}{\left(\xi^{2} k^{2}+\eta^{2} \ell^{2}-\frac{1}{2} d^{2} \xi^{2} k^{2} \eta^{2} \ell^{2}\right)+\left(m^{2}+\frac{1}{4 H^{2}}\right)}
$$

\section{Rossby modes}

Baroclinic modes:

Adding the beta effect to the vorticity equation (1):

$$
\frac{\partial\left(\omega_{z}\right)_{i, j}}{\partial t}=-f_{0} D_{i, j}-\frac{\beta}{f_{0}} \frac{P_{i+1, j}-P_{i-1, j}}{2 d}
$$

Dropping $\partial D / \partial t$ in (2):

$$
0=f_{0}\left(\omega_{z}\right)_{i, j}-\frac{P_{i+1, j+1}+P_{i-1, j+1}+P_{i+1, j-1}+P_{i-1, j-1}-4 P_{i, j}}{2 d^{2}}
$$

Dropping $\partial^{2} D / \partial t^{2}$ in (7):

$$
N^{2} D_{i, j}-\left[\frac{\partial^{2}}{\partial z^{2}}-\left(\frac{1}{2 \rho_{0}} \frac{\partial \rho_{0}}{\partial z}\right)^{2}\right] \frac{\partial}{\partial t} P_{i, j}=0
$$

From (30)-(32):

$$
\begin{aligned}
& -i v \hat{\omega}_{z}=-\mu f_{0} \hat{D}-\frac{\beta}{f_{0}} \frac{e^{i k d}-e^{-i k d}}{2 d} \hat{P} \\
& 0=\left(\xi^{2} k^{2}+\eta^{2} \ell^{2}-\frac{1}{2} d^{2} \xi^{2} k^{2} \eta^{2} \ell^{2}\right) \hat{P}+f_{0} \hat{\omega}_{z}
\end{aligned}
$$




$$
\mu \hat{D} N^{2}-\left[m^{2}+\left(\frac{1}{2 \rho_{0}} \frac{\partial \rho_{0}}{\partial z}\right)^{2}\right] \underset{\sim}{i} v \hat{P}=0
$$

Rewrite (40):

$$
-\underset{\sim}{v} v \hat{\omega}_{z}=-\mu f_{0} \hat{D}-\underset{\sim}{i} \frac{\beta}{f_{0}} \frac{2 \sin (k d)}{2 d} \hat{P}
$$

Rewrite (43):

$$
-\underset{\sim}{i} v \hat{\omega}_{z}=-\mu f_{0} \hat{D}-\underset{\sim}{i} \frac{\beta}{f_{0}} \tilde{\xi}_{k} \hat{P}
$$

where

$$
\tilde{\xi} \equiv \frac{\sin (k d)}{k d}
$$

From (41), (42) and (44):

$$
v N^{2}\left(\xi^{2} k^{2}+\eta^{2} \ell^{2}-\frac{1}{2} d^{2} \xi^{2} k^{2} \eta^{2} \ell^{2}\right) \hat{P}=-f_{0}^{2}\left[m^{2}+\left(\frac{1}{2 \rho_{0}} \frac{\partial \rho_{0}}{\partial z}\right)^{2}\right] v \hat{P}-N^{2} \beta \tilde{\xi} k \hat{P}
$$

Using $\frac{1}{\rho_{0}} \frac{\partial \rho_{0}}{\partial z}=-\frac{1}{H}$ in (46) and for nontrivial solutions $(\hat{P} \neq 0)$ :

$$
v=\frac{-\beta \tilde{\xi}_{k}}{\left(\xi^{2} k^{2}+\eta^{2} \ell^{2}-\frac{1}{2} d^{2} \xi^{2} k^{2} \eta^{2} \ell^{2}\right)+\frac{f_{0}^{2}}{N^{2}}\left(m^{2}+\frac{1}{4 H}\right)}
$$

Barotropic modes:

Assuming $D=0$ in (30)

$$
\frac{\partial\left(\omega_{z}\right)_{i, j}}{\partial t}=-\frac{\beta}{f_{0}} \frac{P_{i+1, j}-P_{i-1, j}}{2 d}
$$

Writing (31) again: 


$$
0=f_{0}\left(\omega_{z}\right)_{i, j}-\frac{P_{i+1, j+1}+P_{i-1, j+1}+P_{i+1, j-1}+P_{i-1, j-1}-4 P_{i, j}}{2 d^{2}}
$$

Assuming $D=0$ in (32)

$$
-\left[\frac{\partial^{2}}{\partial z^{2}}-\left(\frac{1}{2 \rho_{0}} \frac{\partial \rho_{0}}{\partial z}\right)^{2}\right] \frac{\partial}{\partial t} P_{i, j}=0
$$

( $P$ is vertically constant).

From (50) and (51):

$$
0=v\left(\xi^{2} k^{2}+\eta^{2} \ell^{2}-\frac{1}{2} d^{2} \xi^{2} k^{2} \eta^{2} \ell^{2}\right) \hat{P}+\beta \tilde{\xi}_{k} \hat{P}
$$

From (53) and for nontrivial solutions $(\hat{P} \neq 0)$ :

$$
v=\frac{-\beta \tilde{\xi}_{k}}{\xi^{2} k^{2}+\eta^{2} \ell^{2}-\frac{1}{2} d^{2} \xi^{2} k^{2} \eta^{2} \ell^{2}}
$$




\section{Numerical solutions and computational modes}

In this section, we first discuss the construction of linear anelastic numerical models based on the horizontal grids, and then present results from the analyses of the simulations of the inertia-gravity modes on a midlatitude $f$-plane with these models. Our purpose is to confirm the results of the normal mode analyses of the discrete equations, and to investigate the aspects of the horizontal grids, which are not completely disclosed by the normal mode analysis. For better understanding, we categorize the grids into two groups based on the source of the computational modes with them. The first group is comprised of the A-, B-, E-grids, in which the cause of the computational modes is the existence of multiple solutions in the discrete Laplacian operators. The second group includes C-, D-, CD-grids, in which the computational mode is caused by averagings of the variables to each other's grid points. The Z-grid does not fit to any of the two groups because it does not have a computational mode as far as the inertiagravity modes are concerned. For convenience, we include the Z-grid with the discussion of the A, B and E grids.

5.a. Equations of the Z-, A-, B- and E-grid Models

We write the horizontally discrete version equations (2.2)-(2.6) and (2.8) on the Z-, A-, B- and E-grids as 


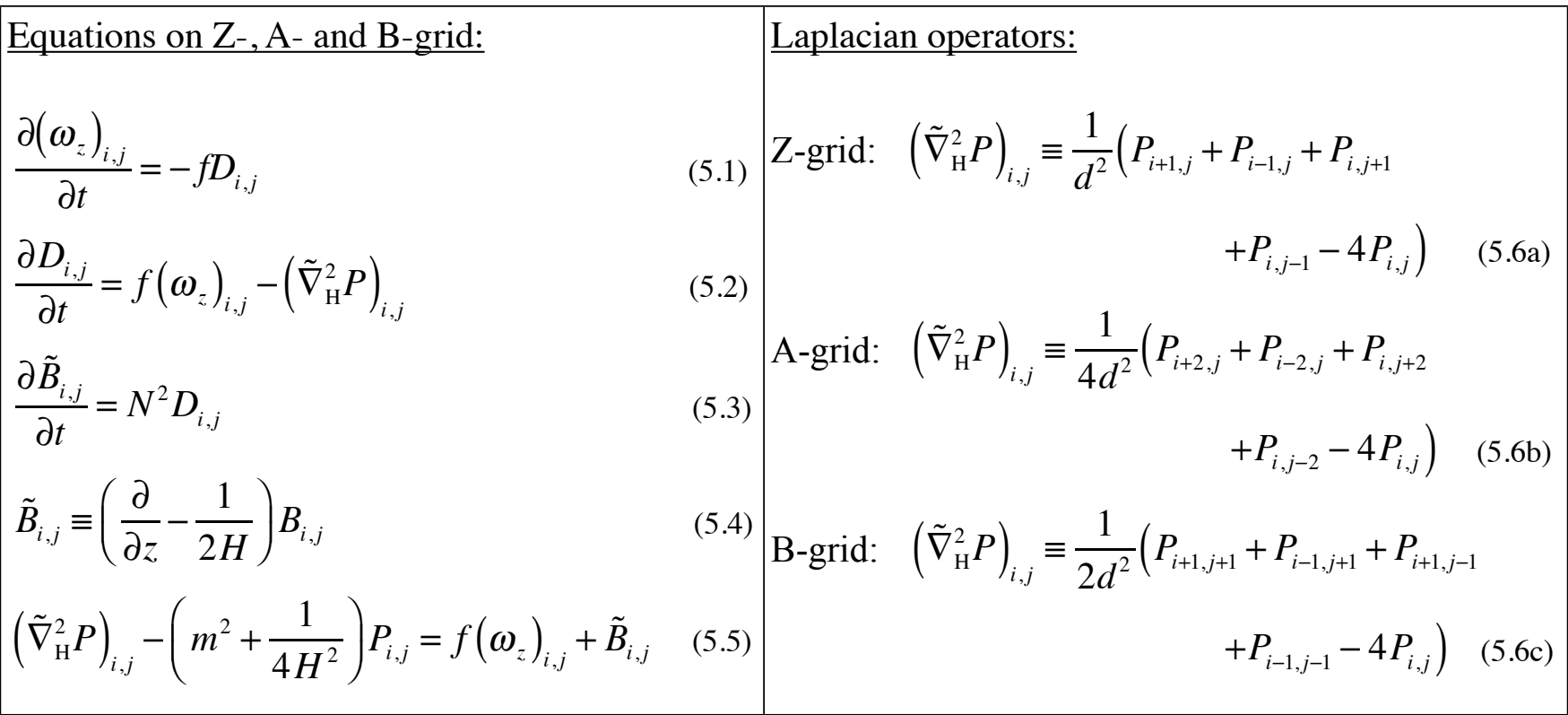

Equations for integer points on E-grid:

$\frac{\partial\left(\omega_{z}\right)_{i, j}}{\partial t}=-f D_{i, j}$

$\frac{\partial D_{i, j}}{\partial t}=f\left(\omega_{z}\right)_{i, j}-\left(\tilde{\nabla}_{\mathrm{H}}^{2} P\right)_{i, j}$

$\frac{\partial \tilde{B}_{i, j}}{\partial t}=N^{2} D_{i, j}$

$\left(\tilde{\nabla}_{\mathrm{H}}^{2} P\right)_{i, j}-\left(m^{2}+\frac{1}{4 H^{2}}\right) P_{i, j}=f\left(\omega_{z}\right)_{i, j}+\tilde{B}_{i, j}$

$\left(\tilde{\nabla}_{\mathrm{H}}^{2} P\right)_{i, j} \equiv \frac{1}{d^{2}}\left(P_{i+1, j}+P_{i-1, j}+P_{i, j+1}+\right.$

$$
\left.P_{i, j-1}-4 P_{i, j}\right)
$$

Equations for half-integer points on E-grid:

$\frac{\partial\left(\omega_{z}\right)_{i+1 / 2, j+1 / 2}}{\partial t}=-f D_{i+1 / 2, j+1 / 2}$ $\frac{\partial D_{i+1 / 2, j+1 / 2}}{\partial t}=f\left(\omega_{z}\right)_{i+1 / 2, j+1 / 2}-\left(\tilde{\nabla}_{\mathrm{H}}^{2} P\right)_{i+1 / 2, j+1 / 2}$

$\frac{\partial \tilde{B}_{i+1 / 2, j+1 / 2}}{\partial t}=N^{2} D_{i+1 / 2, j+1 / 2}$

$\left(\tilde{\nabla}_{\mathrm{H}}^{2} P\right)_{i+1 / 2, j+1 / 2}-\left(m^{2}+\frac{1}{4 H^{2}}\right) P_{i+1 / 2, j+1 / 2}=$

$$
f\left(\omega_{z}\right)_{i+1 / 2, j+1 / 2}+\tilde{B}_{i+1 / 2, j+1 / 2}
$$

$\left(\tilde{\nabla}_{\mathrm{H}}^{2} P\right)_{i+1 / 2, j+1 / 2} \equiv \frac{1}{d^{2}}\left(P_{i+3 / 2, j+1 / 2}+P_{i-1 / 2, j+1 / 2}+\right.$

$$
\left.P_{i+1 / 2, j+3 / 2}+P_{i+1 / 2, j-1 / 2}-4 P_{i+1 / 2, j+1 / 2}\right)
$$

The prognostic variables of the systems are $\omega_{z}, D$ and $\tilde{B}$. The diagnostic variable $P$ is obtained by solving an elliptic equation. In these models, the vertical structure of the numerical solutions is continuous that is prescribed by the vertical wavenumber $m=\pi n / z_{T}$. 
The five-point stencils used in the horizontal finite-differencing of the discrete Laplacian operator in the A, B and $\mathrm{Z}$ grids given by (5.6c), (5.6b) and (5.6c) respectively, differ from each other. This difference greatly impacts the numerical solutions. In the Z-grid, the Laplacian stencil uses information from the neighboring grid points through the cell walls, which leaves no gap in connectivity. The stencils for the neighboring grid points overlaps to participate in the same solution. This is partially the reason why there is no computational mode in the inertia-gravity wave solutions with the Z-grid.

The five-point stencils of the discrete Laplacian operator of the A-grid given by (5.6b) use information from the points that are two-grid distance away, which leave gaps in connectivity. As a result, two separate grid networks are created as shown in Fig. 5.1.a through red or blue colored grid points and dashed lines. No information is exchanged between these networks, and therefore two separate solutions coexist in the A-grid solutions. This is the cause of the computational mode in the Agrid solutions.

The inspection of the five-point stencil on the B-grid shown in Fig.5.1.b indicates that the Laplacian operator uses information from the neighboring points through the corners. The red and blue networks of grid points show the existence of two independent solutions similar to the case with the Agrid, which is the cause of the computational mode in the B-grid solutions.

Now we inspect the stencil on the E-grid. The discrete equations on the E-grid given by (5.7)(5.16) immediately indicate the existence of two separate solutions, one for the cell centers and the other for the cell corners, which are indicated by red and blue grid points and dashed lines in Fig. 5.1.c. As in the A and B grids, the computational mode in the E-grid is also a consequence of the existence of two independent solutions. 
(a)

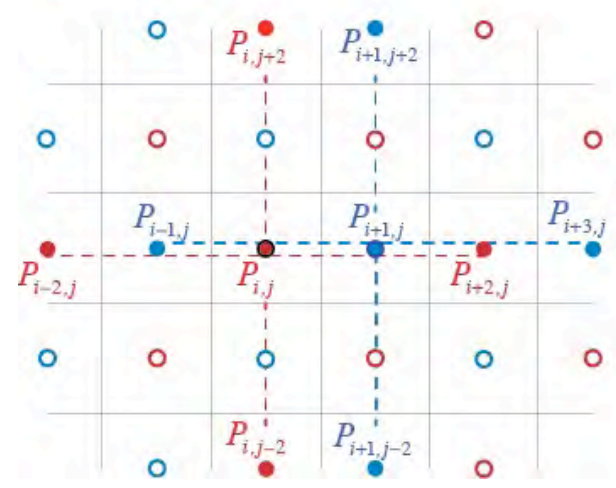

(b)

B-grid

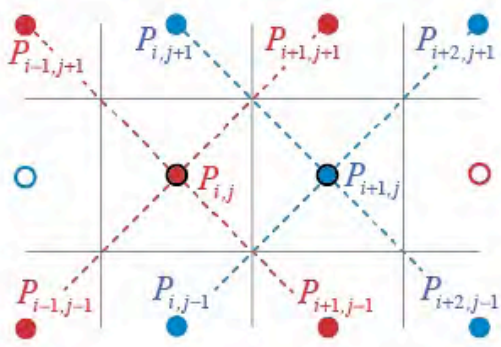

(c) E-grid

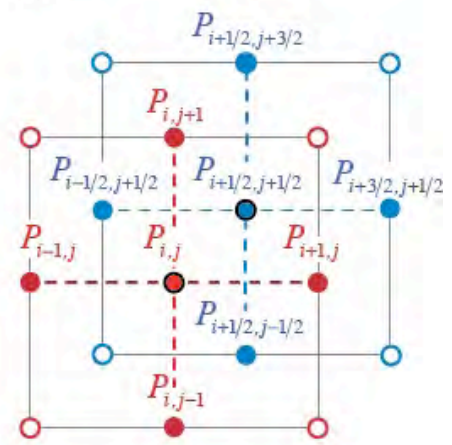

Fig. 5.1.
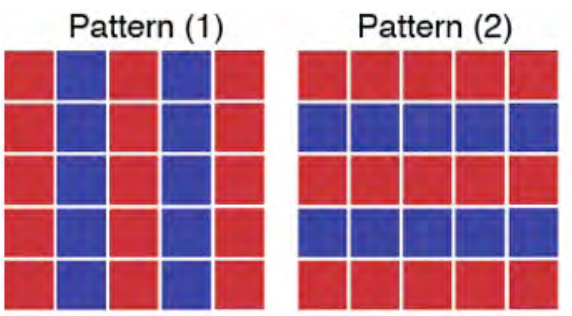

Pattern (3)

Pattern (4)

Pattern (5)
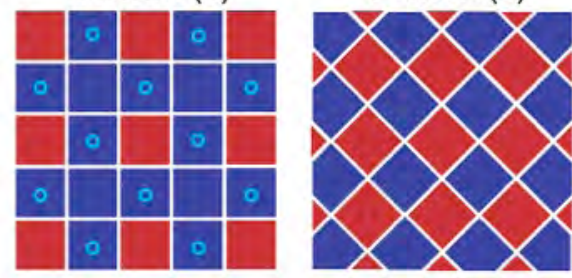

Pattern (6)
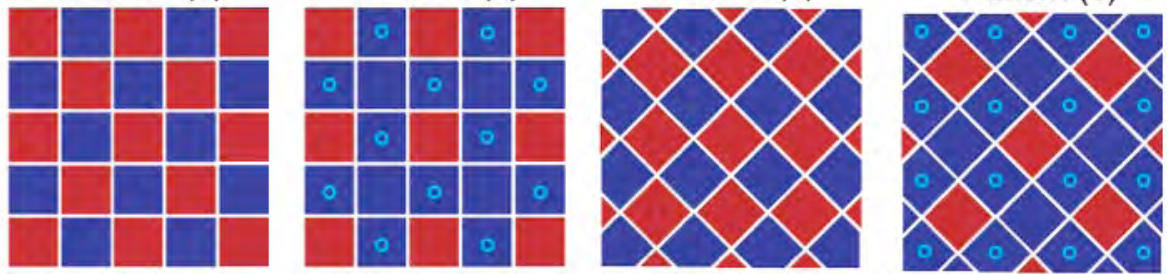

Fig. 5.2.

Based on the discussion above, we identify some perturbation patters that cannot be recognized by the dynamics discretized on the A, B and E grids. Regardless of they are given at the beginning or created later, these perturbation patters remain unchanged in the solutions throughout the integration. Thus, they are called the computational modes. The patterns (1)-(4) shown in Fig.5.2, in which the red and blue denote positive and negative perturbations, respectively, are the computational modes of the Agrid because these patterns of perturbation generate no response from the discrete Laplacian shown in Fig. 5.1.a. The pattern (3) in Fig.5.2 is a computational mode for the B-grid. Since Pattern (3) is included in Pattern (4), the cells with circular markings in the pattern (4) are also computational modes 
for the B-grid. This network of points marked with circles may generate their own solution independent of the solution in the unmarked grid points. The pattern (5) that is a 45-deg rotated version of the Pattern (3) is the computational mode for the E-grid. Recall that the Pattern (3) is a computational mode of the B-grid. Also, the network of cells marked by a circle in the Pattern (6) is a computational mode of the Egrid.

5.c. Equations of the C-, D- and CD-grid Models

We write the horizontally discrete version equations (2.2)-(2.6) and (2.8) on the C- and D-grids as

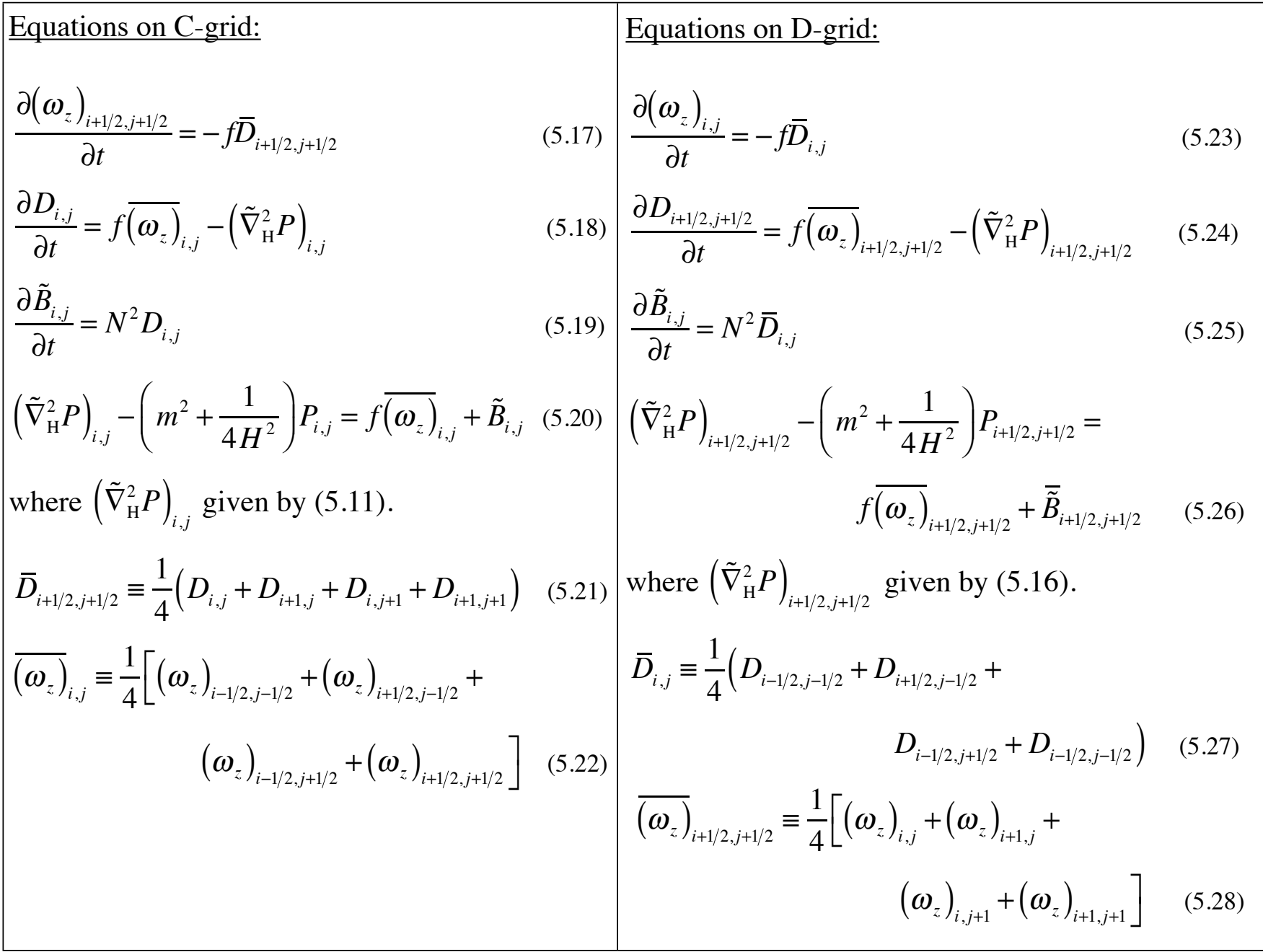


The five-point stencil used by the discrete Laplacian of the C-grid equations is identical to that used in the Z-grid, and therefore it does not cause the problems seen with the A and B grids. The C-grid does neither have the problem seen in the E-grid because there is only one Laplacian in the C-grid. However, the C-grid involves in two averagings used in two equations, one is the averaging of $D$ from centers to the $\omega_{z}$-points at cell corners in the vorticity equation (5.17), and the other the averaging of $\omega_{z}$ from corners to the $D$-points at cell centers in the divergence equation (5.18). The four-point stencils used in the averagings are indicated by the red and blue grid points and arrows in Fig. 5.3.a.

Let us consider the perturbation patterns (1), (2) and (3) for the buoyancy $\tilde{B}$ (or divergence $D$ ) on the C-grid. The existence of such a perturbation given at the cell centers cannot be recognized because of four-point averaging at the cell corners where the vorticity is predicted. These patterns are treated as pure gravity modes, but not as the inertia-gravity modes. Therefore, they are the computational modes of the C-grid.

The D-grid requires four separate four-point averagings used in four equations. The two of these averagings are the averaging of $D$ from corners to the $\omega_{z}$ - and $B$-points at cell centers and the third one is that the averaging of $\omega_{z}$ from the centers to the $D$-points at the corners. The fourth one is that the buoyancy is averaged to the corners to be used on the right hand side of the elliptic equation. In comparison to the $\mathrm{C}$-grid, the $\mathrm{D}$-grid requires two additional averagings in the continuity or thermodynamic equation and in the elliptic equation that determines the pressure $P$. This results in a computational mode that is non-moving or non-oscillating type for the patterns (1), (2) and (3). 
(a)

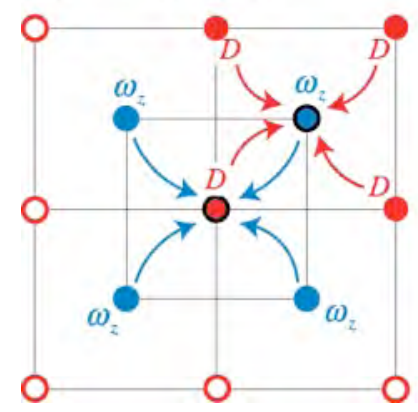

(b) D-grid

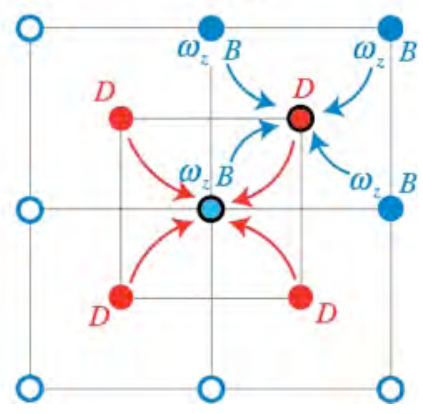

Fig. 5.3.

Now we write the temporally and horizontally discrete version equations (2.2)-(2.6) and (2.8) on the $\mathrm{CD}$-grids as 


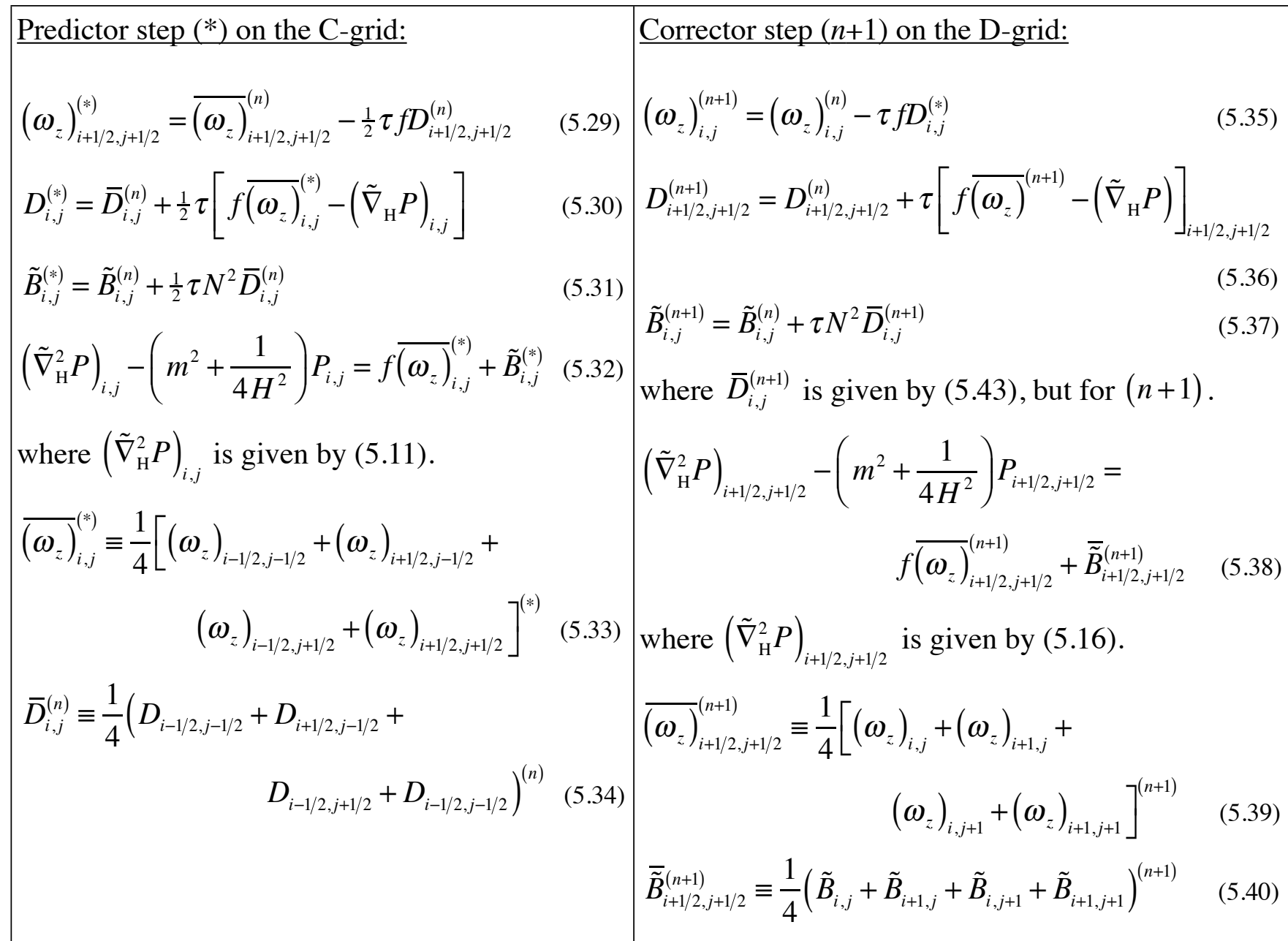

On the CD-grid, the principal prognostic variables that are denoted by $(n)$ and $(n+1)$ reside at the Dgrid, i.e. $\omega_{z}$ and $\tilde{B}$ are placed at the cell centers and $D$ is placed at the cell corners. The variables $\omega_{z}$ and $D$ are averaged to their points on the C-grid at the beginning of the predictor step to predict their provisional values denoted by $(*)$. At the correction step on the D-grid, the provisional value of $D^{(*)}$ is directly used in the prediction of $\omega_{z}$ for the time level $(n+1)$, and all other variables are predicted following a backward scheme, i.e. the latest predicted values on at $(n+1)$ are used in subsequent predictions of other variables.

The patterns of computational modes on the CD-grid are similar to those on the D-grid. Thus, the computational modes on the D-grid are non-moving or non-oscillating type for the patterns (1), (2) and (3). 


\section{5.d. Numerical integrations}

We have integrated the equations of the models based on the different grids described above to validate the normal mode solutions discussed in Section 3 and to illustrate the behavior of computational modes associated with the different grids. For the time discretization of the A-, B-, C- and E-grid models, we have tested different schemes, and have found that the time-integration schemes primarily affect the stability of the solutions, but not the simulated frequency. Among the tested time-integration schemes, a simple forward time-integration scheme that produced neutral solutions with short time steps has been selected.

We first present results from the standing oscillation simulations to validate the frequencies obtained through the normal mode analyses, and also to examine the sensitivity of these simulations to the horizontal grid spacing. The standing oscillations appear stationary because they are produced by the inertial-gravity waves that propagate with the same rate of speed to all directions on the horizontal plane. In these simulations, the vertical structure is continuous, and the vertical integer wavenumber $n$ is prescribed. The simulations start from the initial buoyancy fields shown in Fig. 5.4, for which we have selected the horizontal wave lengths of $L=4 \mathrm{~km}, L=20 \mathrm{~km}, L=50 \mathrm{~km}$ and $L=200 \mathrm{~km}$ in both $x$ - and $y$-directions. These wave lengths correspond to the smallest resolvable horizontal scales used in the frequency plots of the inertia-gravity modes presented in Section 3. The vertical integer wavenumbers used in the simulations are $\mathrm{n}=320,640$ and 1280 for $\mathrm{L}=4 \mathrm{~km} ; \mathrm{n}=160,320$ and 640 for $\mathrm{L}=20 \mathrm{~km} ; \mathrm{n}=80$, $160,320,640$ for $\mathrm{L}=50 \mathrm{~km}$; and $\mathrm{n}=80,160$ and 320 for $\mathrm{L}=200 \mathrm{~km}$. The sensitivity of the numerical solutions to the grid spacing is examined for the three selections: $d=L / 80, d=L / 4$ and $d=L / 2$. The

appearance of the initial buoyancy $\tilde{B}$ field for these three horizontal resolutions is shown in Fig. 5.4. For the highest horizontal resolution $(\mathrm{d}=\mathrm{L} / 80)$ shown in Fig. 5.4.a, the initial waves are well resolved. For the grid spacing $\mathrm{d}=\mathrm{L} / 4$, the initial field that consists of ones, zeros and negative ones appears like alternating upside and inverted "pyramids" due to the poor resolution in the plot shown in Fig. 5.4.b. 
The grid points marked by plus signs $(+)$ are at the positive and negative extremes, the wall centers and the corners of the (upside and inverted) pyramids for this case. For the grid spacing $d=L / 2$, which is the shortest horizontal grid distance to resolve this wave, the initial perturbation also looks like upside and inverted pyramids, but the grid points are only at the extremes of the (upside and inverted) pyramids in this case (Fig. 5.4.c).

(a)

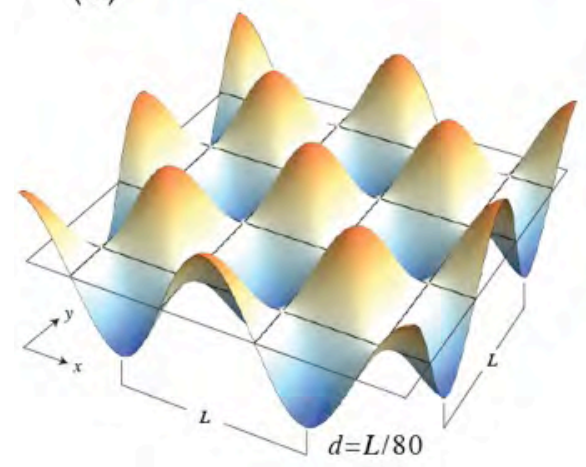

(b)

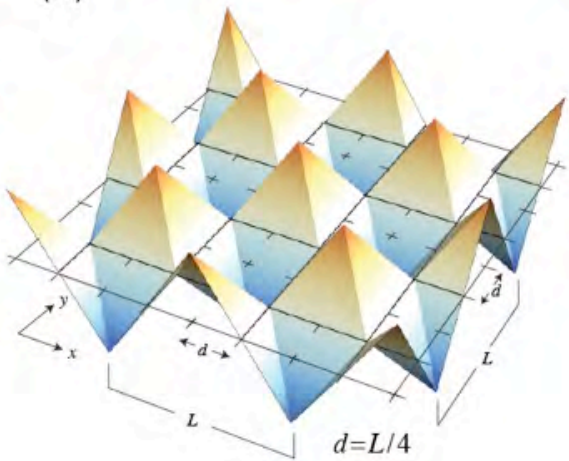

(c)

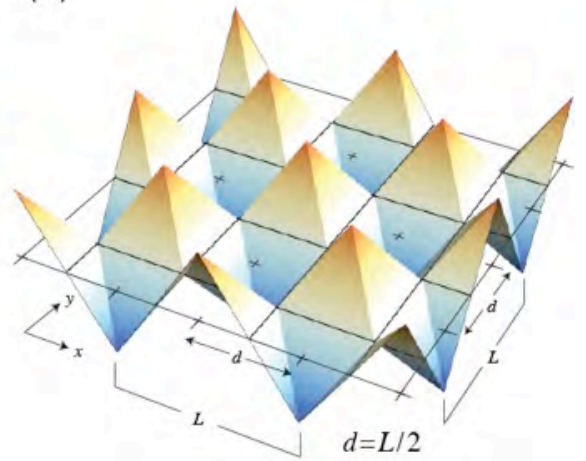

Fig. 5.4 .

The numerical frequencies of the standing oscillations for the high horizontal resolution case $(\mathrm{d}=\mathrm{L} / 80)$ simulated by A-, B- and E-grid models are tabulated in Table 1a. For comparison purposes, we add to the table the true frequency obtained from (2.11) and the numerical frequency obtained by the Zgrid model. Since the simulated is the standing wave, no oscillation is expected at the grid points that have zero perturbation initially. So that, the tabulated results represent the oscillation frequency at the points with non-zero initial perturbation. The numerical oscillation frequencies simulated by the Z-, A-, B-, E-grid models are very close to the true frequencies, which is expected due to the use of high horizontal resolutions in the simulations. All variables oscillate with the same frequency. However, there are still two separate solutions in the A-, B-, E-grid simulations as discusses above. In this case, these two solutions yield the same frequency values because the wavy structure of the initial perturbation is uniform. 
Table 1a.

\begin{tabular}{|c|c|c|c|c|c|c|c|}
\hline \multirow{3}{*}{$\begin{array}{l}\text { Horizontal } \\
\text { scale } \\
\text { of } \\
\text { perturbation }\end{array}$} & \multirow{3}{*}{$\begin{array}{c}\text { Vertical } \\
\text { wavenumber } \\
\text { (Vertically } \\
\text { continuous } \\
\text { solutions) }\end{array}$} & \multirow{2}{*}{$\begin{array}{c}\text { True frequency } v \\
\text { (analytic) } \\
\left(10^{-4} s^{-1}\right)\end{array}$} & \multirow{3}{*}{$\begin{array}{l}\text { Horizontal } \\
\text { grid } \\
\text { distance } \\
(d)\end{array}$} & \multicolumn{4}{|c|}{ Numerical frequency $v\left(10^{-4} s^{-1}\right)$} \\
\hline & & & & Z-grid & A-grid & B-grid & E-grid \\
\hline & & $\begin{array}{ccc}\tilde{B} & D & \omega_{z} \\
\end{array}$ & & $\bar{B} \quad D \quad \omega_{z}$ & $\tilde{B} \quad D \quad \omega_{z}$ & $\tilde{B} \quad D \quad \omega_{z}$ & $\begin{array}{lll}\tilde{B} & D & \omega_{z}\end{array}$ \\
\hline \multirow{3}{*}{$\begin{array}{c}L=4 k m \\
k=\frac{\pi}{2 k m}\end{array}$} & $\begin{array}{l}n=320 \\
\delta z=250 \mathrm{~m}\end{array}$ & 18.84724224 & \multirow{3}{*}{$\begin{array}{c}50 \mathrm{~m} \\
\text { (For Egrid, } \\
70.71 \mathrm{~m} \text { ) }\end{array}$} & 18.84578676 & 18.82320343 & 18.82320343 & 18.84013585 \\
\hline & $\begin{array}{r}n=640 \\
\delta z=125 \mathrm{~m}\end{array}$ & 9.57153193 & & 9.56927399 & 9.56053759 & 9.56053759 & 9.56781682 \\
\hline & $\begin{array}{l}n=1280 \\
\delta z=62.5 \mathrm{~m}\end{array}$ & 4.87709384 & & 4.87597804 & 4.87219704 & 4.87219704 & 4.87559967 \\
\hline \multirow{3}{*}{$\begin{array}{c}L=20 \mathrm{~km} \\
k=\frac{\pi}{10 \mathrm{~km}}\end{array}$} & $\begin{array}{l}n=160 \\
\delta z=500 \mathrm{~m}\end{array}$ & 7.69121581 & \multirow{3}{*}{$\begin{array}{l}250 \mathrm{~m} \\
\text { (For E-grid. } \\
353.55 \mathrm{~m} \text { ) }\end{array}$} & 7.68867511 & 7.68491353 & 7.68491353 & 7.68867511 \\
\hline & $\begin{array}{l}n=320 \\
\delta z=250 m\end{array}$ & 3.94883169 & & 3.94771632 & 3.94474215 & 3.94474215 & 3.94771632 \\
\hline & $\begin{array}{l}n=640 \\
d z=125 m\end{array}$ & 2.15679036 & & 2.15635435 & 2.15502308 & 2.15517092 & 2.15635435 \\
\hline \multirow{2}{*}{$L=50 \mathrm{~km}$} & $\begin{array}{l}n=80 \\
\delta z=1 \mathrm{~km}\end{array}$ & 6.18745719 & & 6.18545511 & 6.18180372 & 6.18058755 & 6.18545511 \\
\hline & $\begin{array}{c}n=160 \\
\delta z=500 m\end{array}$ & 3.21618041 & $625 \mathrm{~m}$ & 3.21555031 & 3.21291946 & 3.21291946 & 3.21555031 \\
\hline \multirow{2}{*}{$k=\frac{\pi}{25 \mathrm{~km}}$} & $\begin{array}{l}n=320 \\
\delta z=250 m\end{array}$ & 1.82684828 & $\begin{array}{l}\text { (For E-gnid, } \\
883.88 \mathrm{~m} \text { ) }\end{array}$ & 1.82650735 & 1.82544605 & 1.82544605 & 1.82650735 \\
\hline & $\begin{array}{l}n=640 \\
\delta z=125 \mathrm{~m}\end{array}$ & 1.25874243 & & 1.25860047 & 1.25824761 & 1.25824761 & 1.25860047 \\
\hline$L=200 \mathrm{~km}$ & $\begin{array}{l}n=80 \\
\delta z=1 \mathrm{~km}\end{array}$ & 1.82682191 & & 1.82650735 & 1.82544605 & 1.82544605 & 1.82650735 \\
\hline$k=\pi$ & $\begin{array}{l}n=160 \\
\delta z=560 m\end{array}$ & 1.25874004 & $\begin{array}{l}2.5 \mathrm{~km} \\
\text { (For E-gnid }\end{array}$ & 1.25860047 & 1.25824761 & 1. 25824761 & 1.25860047 \\
\hline $100 \mathrm{~km}$ & $\begin{array}{l}n=320 \\
\delta z=250 \mathrm{~mm}\end{array}$ & 1.07056681 & $3.53 \mathrm{~km})$ & 1.07053521 & 1.07046226 & 1.07046226 & 1.07053521 \\
\hline
\end{tabular}

Table $1 \mathrm{~b}$ is the follow-up for Table 1a, which shows the numerical frequencies for the $\mathrm{C}, \mathrm{CD}$ and

D grids. The numerical frequencies simulated by the C-, CD-, D-grid models are also very close to the 
Table 1b.

\begin{tabular}{|c|c|c|c|c|c|c|c|}
\hline \multirow{3}{*}{$\begin{array}{l}\text { Horizontal } \\
\text { scale } \\
\text { of } \\
\text { perturbation }\end{array}$} & \multirow{3}{*}{$\begin{array}{c}\text { Vertical } \\
\text { wavenumber } \\
\text { (Vertically } \\
\text { continuous } \\
\text { solutions) }\end{array}$} & \multirow{3}{*}{$\begin{array}{c}\text { True frequency } v \\
\text { (analytic) } \\
\left(10^{-4} s^{-1}\right)\end{array}$} & \multirow{3}{*}{\begin{tabular}{|} 
Horizontal \\
grid \\
distance \\
(d)
\end{tabular}} & \multicolumn{4}{|c|}{ Numerical frequency $v\left(10^{-4} s^{-1}\right)$} \\
\hline & & & & Z-grid & C-grid & CD-grid & D-grid \\
\hline & & & & $\bar{B} \quad D \quad \omega_{2}$ & $\tilde{B} \quad D \quad \omega_{z}$ & $\tilde{B} \quad D \quad \omega_{z}$ & $\begin{array}{llll}\tilde{B} & D & \omega_{z}\end{array}$ \\
\hline \multirow{3}{*}{$\begin{array}{l}L=4 k m \\
k=\frac{\pi}{2 k m}\end{array}$} & $\begin{array}{l}n=320 \\
\delta z=250 \mathrm{~m}\end{array}$ & 18.84724224 & \multirow{3}{*}{$50 \mathrm{~m}$} & 18.84578676 & 18.84578676 & 18.81193205 & 18.81193205 \\
\hline & $\begin{array}{c}n=640 \\
\delta z=125 \mathrm{~m}\end{array}$ & 9.57153193 & & 9.56927399 & 9.56927399 & 9.55472218 & 9.55472218 \\
\hline & $\begin{array}{l}n=1280 \\
\delta z=62.5 \mathrm{~m}\end{array}$ & 4.87709384 & & 4.87597804 & 4.87522137 & 4.86842190 & 4.86842190 \\
\hline \multirow{3}{*}{$\begin{array}{c}L=20 \mathrm{~km} \\
k=\frac{\pi}{10 \mathrm{~km}}\end{array}$} & $\begin{array}{l}n=160 \\
\delta z=500 \mathrm{~m}\end{array}$ & 7.69121581 & \multirow{3}{*}{$250 \mathrm{~m}$} & 7.68867511 & 7.68867511 & 7.67740140 & 7.67740140 \\
\hline & $\begin{array}{l}n=320 \\
\delta_{z}=250 \mathrm{~m}\end{array}$ & 3.94883169 & & 3.94771632 & 3.94771632 & 3.94177246 & 3.94177246 \\
\hline & $\begin{array}{l}n=640 \\
\delta z=125 m\end{array}$ & 2.15679036 & & 2.15635435 & 2.15561455 & 2.15310304 & 2.15310304 \\
\hline$t-50 / m$ & $\begin{array}{l}n=80 \\
\delta z=1 \mathrm{~km}\end{array}$ & 6.18745719 & & 6.18545511 & 6.18423750 & 6.17572764 & 6.17572764 \\
\hline L- & $\begin{array}{l}n=160 \\
\delta z=500 \mathrm{~m}\end{array}$ & 3.21618041 & $625 \mathrm{~m}$ & 3.21555031 & 3.21489219 & 3.21062100 & 3.21062100 \\
\hline$k=\frac{\pi}{25 k m}$ & $\begin{array}{l}n=320 \\
\delta z=250 m\end{array}$ & 1.82684828 & $0<2 \mathrm{~m}$ & 1.82650735 & 1.82565821 & 1.82364465 & 1.82364465 \\
\hline & $\begin{array}{l}n=640 \\
\delta z=125 \mathrm{~m}\end{array}$ & 1.25874243 & & 1.25860047 & 1.25739149 & 1.25668732 & 1.25668732 \\
\hline$L=200 \mathrm{~km}$ & $\begin{array}{l}n=80 \\
\delta z=1 \mathrm{~km}\end{array}$ & 1.82682191 & & 1.82650735 & 1.82565821 & 1.82364465 & 1.82364465 \\
\hline$k=\frac{\pi}{n}$ & $\begin{array}{l}n=160 \\
\delta z=500 \mathrm{~m}\end{array}$ & 1.25874004 & $2.5 \mathrm{~km}$ & 1.25860047 & 1.25739149 & 1.25668732 & 1.25668732 \\
\hline $100 \mathrm{~km}$ & $\begin{array}{l}n=320 \\
d z=250 \mathrm{~m}\end{array}$ & 1.07056681 & & 1. 07053521 & 1.06907801 & 1. 06885977 & 1.06885977 \\
\hline
\end{tabular}

true frequencies as expected due to the use of high horizontal resolutions in the simulations. All variables oscillate with the same frequency, and there is only one solution in the solutions with these grids. It is a noticeable fact that the numerical frequencies obtained by the CD-grid is identical to ones obtained by the D-grid.

We have repeated the same simulation by using lower horizontal resolutions down to (d $>$ L/4), and found no unexpected differences between the high and lower resolution simulations with the exception that the accuracy of the numerically calculated frequency degrades with lowering resolution. For the horizontal resolution $(\mathrm{d}=\mathrm{L} / 4)$, which is the half of the shortest spacing to resolve the initial perturbation, the effect of non-moving and non-oscillating computational modes with the A, B and E grids can be identified easier than the high resolution cases. Table 2 a tabulates the true frequency and numerical frequencies obtained by the Z-, A-, B- and E-grids for the horizontal resolution (d=L/4). All variables at all the grid points oscillate with the same frequency in the Z-grid simulation. Obviously, the 
grid points that have zero perturbation do not show oscillation because of the standing wave simulation. If the horizontal distribution of the initial perturbation is modified, all the grid points physically respond to the change. The numerically obtained frequency by the Z-grid is generally smaller than the true frequency, but it is close. In the table, the non-moving and non-oscillating solutions that corresponds to the computational mode is indicated with red numbers. In A-, B- and E-grids, all grid points at the corners of the "pyramids" in Fig. 5.4b that have zero perturbation initially produce no oscillation even with the case that the perturbation values in the other grid points are modified. The solutions at the other points for short horizontal scale perturbations yield much smaller frequencies than the Z-grid frequency.

The numerical frequencies obtained by the C-, CD- and D-grid models for the same horizontal resolution are tabulated in Table $2 \mathrm{~b}$. The table shows that all variables of the modes are oscillating with the same frequency, but some of the modes are oscillating with lower frequency than that of the inertial frequency (blue numbers), which indicates that these modes cannot recognize the rotation properly due to the averaging of the divergence and vorticity to each other's grid points. The modes with the short horizontal scales simulated with the $\mathrm{C}$-grid have frequencies similar to those obtained by the Z-grid. The $\mathrm{CD}$ - and D-grid produce almost identical frequencies, which are much lower than those produces by the Z- and C-grids.

Finally we tabulate results obtained by using the shortest possible horizontal grid spacing ( $d=\mathrm{L} /$ 2) to resolve the initial perturbation shown Fig. 5.4c in Table 3. This is the horizontal resolution that the errors due to finite-differencing is the highest, and the computational modes impact the solutions the most. It is evident from the non-oscillating solutions (indicated by red zeros in Table 3) that the A-, Band E-grid simulations cannot recognize the initial buoyancy perturbations because the two separate networks with these grids recognize the initial perturbation as horizontally uniform fields. However, if the initial perturbation is given to the vorticity instead of the buoyancy, the solutions are oscillatory with the frequency of inertial oscillation (indicated by the green numbers in Table 3), which is the solution that the normal mode analysis produces for the SRHS. The D- and CD-grid solutions do not yield oscillation either, but the reason for this is different from the A-, B- and E-grid cases. Averaging of the initial buoyancy perturbation to the divergence points completely wipes out the wave in the D- and CDgrids. Thus, the initial perturbation pattern is the computational mode, which is identical to the Pattern (3) in Fig 5.2. Non-oscillating solutions are also obtained by starting from the vorticity perturbations as 
indicated by green zeros in Table 3 . In the C-grid solution, the buoyancy and divergence recognizes the initial perturbation and they produce oscillations although the vorticity is decoupled from the others and it does not oscillate. This is due to the averaging of the divergence to the vorticity points, which wipes out the initial perturbation at the vorticity points. For the short horizontal scales, the frequencies of the buoyancy and divergence in the $\mathrm{C}$-grid solutions is very close to those in the Z-grid solutions. For the long horizontal and short vertical scales, the frequency of buoyancy and divergence in the C-grid solutions is considerably smaller than that in the Z-grid solutions and rotational frequency (indicated by blue numbers in Table 3). If the initial perturbation is given to the vorticity instead of the buoyancy in the $\mathrm{C}, \mathrm{CD}$ and $\mathrm{D}$ grid simulations, the solutions are non-oscillatory for all variables (indicated by the green numbers in Table 3). The Z-grid solution produces numerical frequencies that are very close to the true frequencies although the initial perturbation is poorly resolved. 
Table 2a.

\begin{tabular}{|c|c|c|c|c|c|c|c|}
\hline \multirow{3}{*}{$\begin{array}{l}\text { Horizontal } \\
\text { scale } \\
\text { of } \\
\text { perturbation }\end{array}$} & \multirow{3}{*}{$\begin{array}{c}\text { Vertical } \\
\text { wavenumber } \\
\text { (Vertically } \\
\text { continuous } \\
\text { solutions) }\end{array}$} & \multirow{2}{*}{$\begin{array}{c}\text { True frequency } v \\
\text { (analytic) } \\
\left(10^{-4} s^{-1}\right)\end{array}$} & \multirow{3}{*}{$\begin{array}{c}\text { Horizontal } \\
\text { grid } \\
\text { distance } \\
(d)\end{array}$} & \multicolumn{4}{|c|}{ Numerical frequency $v\left(10^{-4} s^{-1}\right)$} \\
\hline & & & & Z-grid & A-grid & B-grid & E-grid \\
\hline & & $\tilde{B} \quad D \quad \omega_{z}$ & & $\tilde{B} \quad D \quad \omega_{z}$ & $\tilde{B} \quad D \quad \omega_{z}$ & $\tilde{B} \quad D \quad \omega_{z}$ & $\tilde{B} \quad D \quad \omega_{1}$ \\
\hline \multirow[t]{3}{*}{$L=4 / m$} & $\begin{array}{l}n=320 \\
\delta z=250 \mathrm{~m}\end{array}$ & 18.84724224 & \multirow{3}{*}{$\begin{array}{c}1 \mathrm{~km} \\
\text { (For E-grid, } \\
1.41 \mathrm{~km} \text { ) }\end{array}$} & 17.01837840 & $\begin{array}{c}12.13438645 \\
0 . \emptyset\end{array}$ & $\begin{array}{c}12.13438645 \\
0 . \emptyset\end{array}$ & $\begin{array}{c}12.13204345 \\
0.0\end{array}$ \\
\hline & $\begin{array}{c}n=640 \\
\delta z=125 m\end{array}$ & 9.57153193 & & 8.63549382 & $\begin{array}{c}6.15636420 \\
0.0\end{array}$ & $\begin{array}{c}6.15636420 \\
0.0\end{array}$ & $\begin{array}{c}6.15576105 \\
0.0\end{array}$ \\
\hline & $\begin{array}{l}n=1280 \\
\delta z=62.5 \mathrm{~m}\end{array}$ & 4.87709384 & & 4.41296903 & $\begin{array}{c}3.20080759 \\
0.0\end{array}$ & $\begin{array}{c}3.20080759 \\
0.0\end{array}$ & $\begin{array}{c}3.20097065 \\
0.0\end{array}$ \\
\hline \multirow[t]{2}{*}{$L=20 \mathrm{~km}$} & $\begin{array}{l}n=160 \\
\delta z=500 m\end{array}$ & 7.69121581 & \multirow{3}{*}{$\begin{array}{l}5 \mathrm{~km} \\
\text { (For E-grid. } \\
7.07 \mathrm{~km} \text { ) }\end{array}$} & 6.94121222 & $\begin{array}{c}4.96380574 \\
\emptyset . \emptyset\end{array}$ & $\begin{array}{c}4.96380574 \\
6.0\end{array}$ & $\begin{array}{c}4.96341362 \\
0.0\end{array}$ \\
\hline & $\begin{array}{l}n=320 \\
\delta z=250 m\end{array}$ & 3.94883169 & & 3.58220370 & $\begin{array}{c}2.63026846 \\
0.0\end{array}$ & $\begin{array}{c}2.63026846 \\
0.0\end{array}$ & $\begin{array}{c}2.63026846 \\
0.0\end{array}$ \\
\hline$\kappa=\overline{10 k m}$ & $\begin{array}{l}n=640 \\
\delta z=125 m\end{array}$ & 2.15679036 & & 1.98998711 & $\begin{array}{c}1.57489104 \\
\emptyset . \emptyset\end{array}$ & $\begin{array}{c}1.57489104 \\
0.0\end{array}$ & $\begin{array}{c}1.57485156 \\
0.0\end{array}$ \\
\hline \multirow{4}{*}{$\begin{array}{c}L=50 \mathrm{~km} \\
k=\frac{\pi}{25 \mathrm{~km}}\end{array}$} & $\begin{array}{l}n=80 \\
\delta z=1 \mathrm{~km}\end{array}$ & 6.18745719 & \multirow{4}{*}{$\begin{array}{l}12.5 \mathrm{~km} \\
\text { (For E-grid, } \\
17.67 \mathrm{~km} \text { ) }\end{array}$} & 5.58902802 & $\begin{array}{c}4.01738191 \\
0.0\end{array}$ & $\begin{array}{c}4.01738191 \\
0.0\end{array}$ & $\begin{array}{c}4.01738191 \\
0.0\end{array}$ \\
\hline & $\begin{array}{l}n=160 \\
\delta z=500 m\end{array}$ & 3.21618041 & & 2.92840478 & $\begin{array}{c}2.18834818 \\
0.0\end{array}$ & $\begin{array}{c}2.18834818 \\
\emptyset . \emptyset\end{array}$ & $\begin{array}{c}2.18827196 \\
0.0\end{array}$ \\
\hline & $\begin{array}{l}n=320 \\
\delta z=250 \mathrm{~m}\end{array}$ & 1.82684828 & & 1.70137701 & $\begin{array}{c}1.39551912 \\
0.0\end{array}$ & $\begin{array}{c}1.39551912 \\
0.0\end{array}$ & $\begin{array}{c}1.39548813 \\
\emptyset .0\end{array}$ \\
\hline & $\begin{array}{l}n=640 \\
\delta z=125 m\end{array}$ & 1.25874243 & & 1.21395442 & $\begin{array}{c}1.11214693 \\
0 . \emptyset\end{array}$ & $\begin{array}{c}1.11214693 \\
0 . \emptyset\end{array}$ & $\begin{array}{c}1.11211272 \\
\emptyset .0\end{array}$ \\
\hline \multirow{3}{*}{$\begin{array}{l}L=200 \mathrm{~km} \\
k=\frac{\pi}{100 \mathrm{~km}}\end{array}$} & $\begin{array}{l}n=80 \\
\delta z=1 \mathrm{~km}\end{array}$ & 1.82682191 & \multirow{3}{*}{$\begin{array}{l}50 \mathrm{~km} \\
\text { (For E-grid, } \\
70.71 \mathrm{~km} \text { ) }\end{array}$} & 1.70137701 & $\begin{array}{c}1.39545713 \\
\varnothing . \varnothing\end{array}$ & $\begin{array}{c}1.39545713 \\
0.0\end{array}$ & $\begin{array}{c}1.39545713 \\
0.0\end{array}$ \\
\hline & $\begin{array}{l}n=160 \\
\delta z=500 m\end{array}$ & 1.25874004 & & 1.21395442 & $\begin{array}{c}1.11214693 \\
\emptyset . \emptyset\end{array}$ & $\begin{array}{c}1.11214693 \\
0.0\end{array}$ & $\begin{array}{c}1.11211272 \\
0.0\end{array}$ \\
\hline & $\begin{array}{l}n=320 \\
\delta z=250 \mathrm{~m}\end{array}$ & 1.07056681 & & 1.05756165 & $\begin{array}{c}1.02918678 \\
0,0\end{array}$ & $\begin{array}{c}1.02918678 \\
0.0\end{array}$ & $\begin{array}{c}1.02916992 \\
0.0\end{array}$ \\
\hline
\end{tabular}


Table 2b.

\begin{tabular}{|c|c|c|c|c|c|c|c|}
\hline \multirow{3}{*}{$\begin{array}{c}\text { Horizontal } \\
\text { scale } \\
\text { of } \\
\text { perturbation }\end{array}$} & \multirow{3}{*}{$\begin{array}{c}\text { Vertical } \\
\text { wavenumber } \\
\text { (Vertically } \\
\text { continuous } \\
\text { solutions) }\end{array}$} & \multirow{2}{*}{$\begin{array}{c}\text { True frequency } V \\
\text { (analytic) } \\
\left(10^{-4} s^{-1}\right)\end{array}$} & \multirow{3}{*}{$\begin{array}{l}\text { Horizontal } \\
\text { grid } \\
\text { distance } \\
\text { (d) }\end{array}$} & \multicolumn{4}{|c|}{ Numerical frequency $v\left(10^{-4} s^{-1}\right)$} \\
\hline & & & & Z-grid & C-grid & CD-grid & D-grid \\
\hline & & $\tilde{B} \quad D \quad \omega_{z}$ & & $\begin{array}{lll}\bar{B} & D & \omega_{z} \\
\end{array}$ & $\tilde{B} \quad D \quad \omega_{z}$ & $\begin{array}{lll}\tilde{B} & D & \omega_{z} \\
\end{array}$ & $\tilde{B} \quad D \quad \omega_{z}$ \\
\hline \multirow{3}{*}{$\begin{array}{l}L=4 k m \\
k=\frac{\pi}{2 k m}\end{array}$} & $\begin{array}{l}n=320 \\
\delta z=250 m\end{array}$ & 18.84724224 & \multirow{3}{*}{$1 \mathrm{~km}$} & 17.01837840 & 16.99996024 & 8.51149459 & 8.51149459 \\
\hline & $\begin{array}{r}n=640 \\
\delta z=125 m\end{array}$ & 9.57153193 & & 8.63549382 & 8.59062798 & 4. 31715357 & 4.31715357 \\
\hline & $\begin{array}{l}n=1280 \\
\delta z=62.5 \mathrm{~m}\end{array}$ & 4.87709384 & & 4.41296903 & 4.32726260 & 2.20663949 & 2.20663949 \\
\hline \multirow{3}{*}{$k=\frac{\pi}{10 \mathrm{~km}}$} & $\begin{array}{l}n=160 \\
\delta z=500 \mathrm{~m}\end{array}$ & 7.69121581 & \multirow{3}{*}{$5 \mathrm{~km}$} & 6.94121222 & 6.88794705 & 3.47098956 & 3.47060611 \\
\hline & $\begin{array}{l}n=320 \\
\delta z=250 \mathrm{ml}\end{array}$ & 3.94883169 & & 3.58220370 & 3.47598213 & 1.79110185 & 1.79110185 \\
\hline & $\begin{array}{l}n=640 \\
\delta z=125 \mathrm{~m}\end{array}$ & 2.15679036 & & 1.98998711 & 1.79171475 & 0.99499355 & 0.99499355 \\
\hline \multirow{2}{*}{$L=50 \mathrm{~km}$} & $\begin{array}{l}n=80 \\
\delta z=1 \mathrm{~km}\end{array}$ & 6.18745719 & \multirow{4}{*}{$12.5 \mathrm{~km}$} & 5.58902802 & 5.522222298 & 2.79451401 & 2.79476261 \\
\hline & $\begin{array}{c}n=160 \\
\delta z=500 \mathrm{~m}\end{array}$ & 3.21618041 & & 2.92840478 & 2.79750013 & 1.46420239 & 1.46413415 \\
\hline$k=\frac{\pi}{25 \mathrm{~km}}$ & $\begin{array}{l}n=320 \\
\delta z=250 \mathrm{~m}\end{array}$ & 1.82684828 & & 1.70137701 & 1.46454368 & 0.85068850 & 0.85068850 \\
\hline $25 K \pi$ & $\begin{array}{l}n=640 \\
\delta z=125 \mathrm{~m}\end{array}$ & 1.25874243 & & 1.21395442 & 0.85073457 & 0.69701239 & 0.60698893 \\
\hline$L=200 \mathrm{~km}$ & $\begin{array}{l}n=80 \\
\delta z=1 \mathrm{~km}\end{array}$ & 1.82682191 & & 1.70137701 & 1.46447541 & 0.85068850 & $\emptyset .85066547$ \\
\hline$k=\frac{\pi}{1}$ & $\begin{array}{l}n=160 \\
\delta z=500 m\end{array}$ & 1.25874004 & $50 \mathrm{~km}$ & 1.21395442 & 0.85073457 & 0.60700066 & ๑. 60698893 \\
\hline $100 \mathrm{~km}$ & $\begin{array}{l}n=320 \\
\delta z=250 m\end{array}$ & 1. 07056681 & & 1.05756165 & 0.60700066 & 0.52879862 & 0.52878082 \\
\hline
\end{tabular}


Table 3.

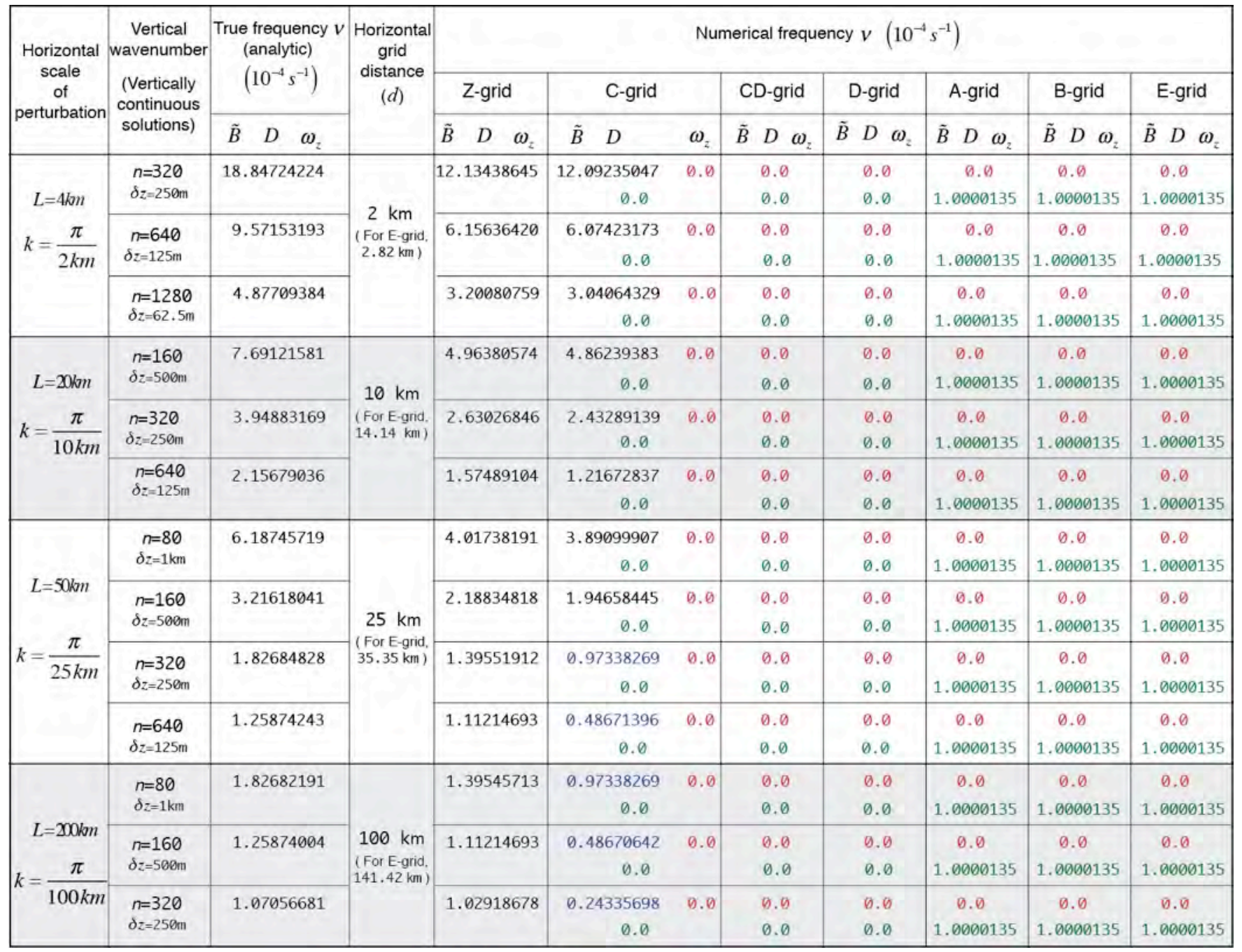

We have also made simulations to demonstrate the behavior of the computational modes during the propagation of inertia-gravity modes with the seven grids we are discussing in this paper. These simulations start from a gaussian buoyancy perturbation with a positive amplitude placed in the middle of the horizontal domain with rapidly decaying amplitude away from the center. To superimpose a gridscale noise on the initial condition, the perturbation at every other grid point is set to zero. The horizontal domain is 280 by 280 grid points, the horizontal grid spacing is $\mathrm{d}=50 \mathrm{~m}$ (for E-grid $\mathrm{d}=70.71$ $\mathrm{m})$. The radius of the perturbation that is the distance between the peak of the perturbation to the distance where the perturbation becomes zero is $950 \mathrm{~m}$. In the vertical, the perturbation is continuous with the vertical integer wavenumber of $n=320$. The buoyancy field after 100 mins of integration in the 
124-by-124 wide corner-end portion of the horizontal domain is shown in Fig. 5.5. For reference purposes, we show Z-grid result obtained without the superimposed grid-scale noise on the initial perturbation. No major difference can be seen between the Z-grid solutions started with the computational mode and without it in the portion of the domain shown in Fig 5.5. In the solution with the grid-scale noise, there is, however, a remnant of the initial noise taking a form of grid-scale standing inertia-gravity oscillation at and near the center of the domain where the peak of the initial perturbation is placed. This is not visible in Fig. 5.5 because it is out of the plot domain. The noise gradually subsides in time as the bulk of the initial perturbation propagate outward and vacate the center of the domain. The $\mathrm{C}$-grid simulation result is very close to the Z-grid result with the initial noise. It is evident that the existence of initial noise does not leave a permanent effect on the solutions with the Z- and C-grids. The $\mathrm{CD}$ - and D-grid simulation results resemble the C-grid result with an exception that the noise is apparent near the center of the domain in both simulations (see Fig. 5.5). Since the noise triggers the computational mode, it tends to stay for a long time in the CD- and D-grid simulations. By prescribing the initial perturbation at every other grid point to zero, one of the two independent solutions in the A-, B- and E-grid simulations is set to zero. The other solution in these grids recognizes the initial gaussian perturbation and yields a propagating wave as shown in the last three figures of the lower panel of Fig. 5.5 similar to those with the Z-, C-, CD- and D-grids.

In summary, the $\mathrm{Z}$ grid is the only grid, which generates unique solutions in the three numerical simulations discussed in this section. Also, the simulated frequencies with the $\mathrm{Z}$ grid are remarkably accurate in the sense that they are very close to the true frequencies. The numerical simulations with the $\mathrm{C}$ grid are overall very close to those with the $\mathrm{Z}$ grid. However, the $\mathrm{C}$ grid generates multiple solutions with all horizontal grid resolutions for the modes with the SRHS. The D and CD grid results are virtually identical to each other. The modes with the SRHS regardless of the variable are completely unrecognized by the $\mathrm{D}$ and $\mathrm{CD}$ grid. The $\mathrm{A}, \mathrm{B}$ and $\mathrm{E}$ grids have multiple solutions for all resolutions, and, they produce mixed solutions depending on the variable for the SRHS. 


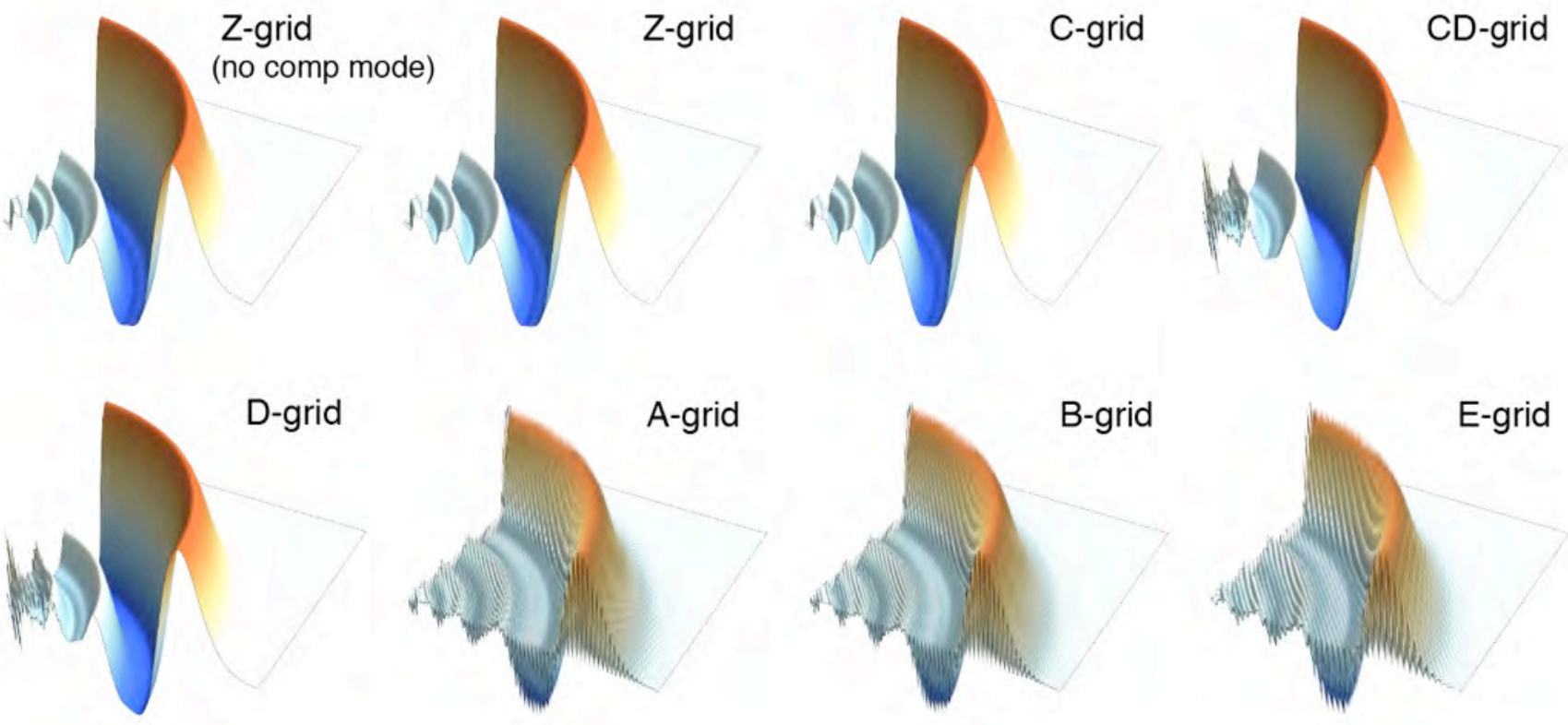

Fig. 5.5. 


\section{Summary and Conclusions}

We have discussed the horizontal and vertical discretization of the nonhydrostatic linearized anelastic equations on the $\mathrm{A}, \mathrm{B}, \mathrm{C}, \mathrm{CD}, \mathrm{D}, \mathrm{E}$, and $\mathrm{Z}$ horizontal grids and the $\mathrm{L}$ and $\mathrm{CP}$ vertical grids, with an emphasis on the middle-latitude inertia-gravity and Rossby waves. We have followed a two tier approach in our analyses. The impact of the discretization on the physical modes is demonstrated through comparisons of the normal mode analyses applied to the continuous and discrete equations. The source and behavior of computational modes in the horizontally discrete systems are mostly investigated by the analyses of the numerical solutions obtained by the models based on these grids and, small part, by the normal mode analysis to the extent deemed possible.

The $\mathrm{Z}$ grid yields the closest inertia-gravity wave dispersion to the true solutions among the seven horizontal grids we considered in this paper without any computational modes. Although the frequency and group velocity of inertia-gravity modes in the Z-grid solutions are lower than the true ones, the numerical frequency never goes below the inertial frequency and the group velocity never reverses. In the Z-grid solution of the quasi-geostrophic baroclinic and barotropic Rossby waves, there is, however, a non-oscillating computational mode appears. The shortest horizontal zonal scale cannot recognize the beta effect, which generates a non-oscillating computational mode.

The $\mathrm{C}$ grid produces mixed dispersion results in the inertia-gravity solutions. For the cloudresolving applications with small horizontal grid spacing represented by $d=2 \mathrm{~km}$ in our analyses, the accuracy of the physical modes are nearly identical to that of the physical modes with the Z-grid unless very high vertical resolutions are used. Of course, there is a computational mode that decouples the divergence and vorticity for the SRHS in the C-grid solution. The vorticity and divergence are placed at different grid points on the C-grid. Thus, averagings of the vorticity and divergence to each other's grid points leads to the computational mode. The impact of this computational mode may not be severe in a linear system because this mode disperses like a pure gravity mode, which minimizes the potential problems. However, since the three-dimensional enstrophy cascades to the SRHS in the nonlinear cloudresolving models, the impact of the computational mode can be severe, and an explicit process may be needed to damp the enstrophy accumulated in the SRHS. For the mesoscale applications, i.e. horizontal 
grid spacing represented by $10 \mathrm{~km}$ and $25 \mathrm{~km}$, the inertia-gravity modes in the C-grid solution behave similar to that in the Z-grid solutions if the vertical wavenumbers are equal to $n=320$ and $n=156$ or smaller for $10 \mathrm{~km}$ and $25 \mathrm{~km}$ grid spacings, respectively. The wavenumbers $n=320$ and $n=156$ correspond to $\delta z=250 \mathrm{~m}$ and $\delta z=512 \mathrm{~m}$ for the domain height of $z_{T}=80 \mathrm{~km}$, respectively. The inertia-gravity modes in the C-grid solutions with a typical climate model horizontal grid spacing of 100 $\mathrm{km}$ are simulated as accurate as the Z-grid solutions for the vertical wavenumbers $n=39$ and below, which corresponds to $\delta z=2051 \mathrm{~m}$ and higher. For the vertical wavenumbers higher than $n=39$, the reversal of group velocity takes place for the modes with rather wide range of horizontal scales causing stability problems.

The performance of the C-grid in simulating middle-latitude Rossby waves is comparable to the Z-grid. In summary, the C-grid performs the best in the horizontally high resolutions models, and it yields as accurate physical solutions as the Z-grid. The computational mode impacts the solutions less for the high resolution applications than the low resolution ones.

The inertia-gravity and Rossby mode solutions with the $\mathrm{D}$ and CD grids are almost identical. On these grids, the divergence and the mass (buoyancy, pressure and vertical velocity) are placed in different grid points. The vorticity is placed at the same grid points with the mass. With this staggering, not only the vorticity and divergence are averaged to each other's grid points, but also the divergence is averaged to mass (and vertical velocity) points and the pressure is averaged to divergence points. The result is the large errors in the dispersion of the inertia-gravity modes of all vertical scales near the SRHS for all horizontal resolutions. At the SRHS, there are non-moving and non-oscillating computational modes of all variables. The group velocity reverses with a high rate of speed near the SRHS. The D and CD grids may require explicit diffusion in nonlinear models to clear the noise in short horizontal scales due to the computational mode and reversal of the group velocity. The $\mathrm{D}$ and $\mathrm{CD}$ grids along with the $\mathrm{Z}$ grid produce the best dispersion of the middle-latitude Rossby waves among all the horizontal grids.

The dispersions of the inertia-gravity waves with the A, B and E grids are similar. All suffer from the existence of double physical solutions that are independent of each other as demonstrated by the 
numerical simulations. To avoid the separation between these two solutions, a horizontal mixing process is needed with these grids. The computational modes with the SRHS display a complicated behavior. There are two possible solutions, one of which is non-oscillating computational mode, and the other is a different computational mode that oscillates with the inertial frequency. 


\section{DC-grid}
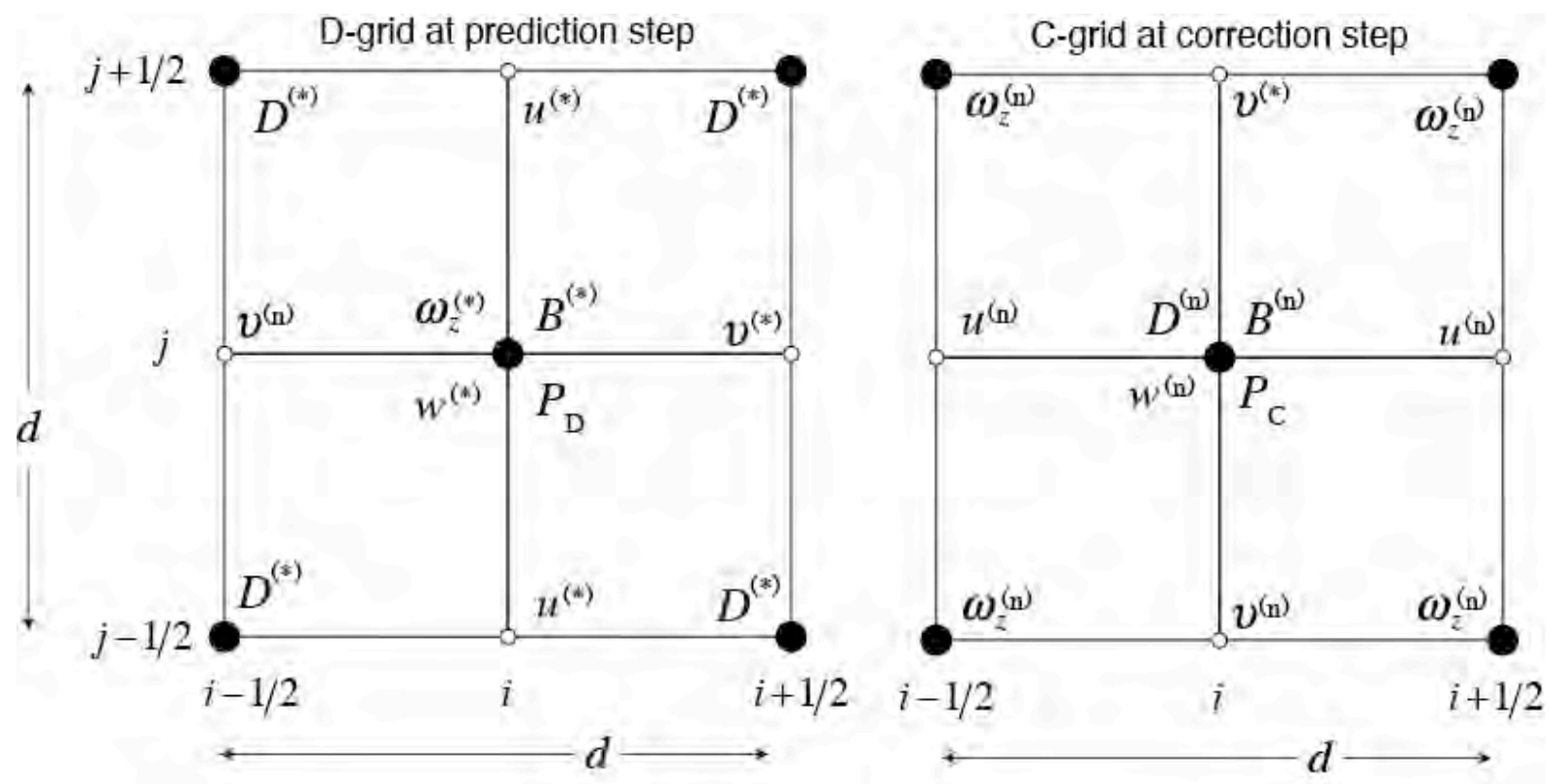

The DC-grid

Normal mode analysis for the DC-grid (Inertia-gravity modes):

\begin{tabular}{|c|c|c|c|}
\hline \multicolumn{2}{|l|}{ Predictor step $(*)$ on the D-grid: } & \multicolumn{2}{|l|}{ Corrector step $(n+1)$ on the $C$-grid: } \\
\hline$\hat{\omega}_{z}^{(*)}=\mu \hat{\omega}_{z}^{(n)}-\frac{1}{2} \tau f \hat{D}^{(n)}$ & (DC.1) & $\hat{\omega}_{z}^{(\mathrm{n}+1)}=\hat{\omega}_{z}^{(\mathrm{n})}-\tau f \hat{D}^{(*)}$ & (DC.6) \\
\hline$\hat{D}^{(*)}=\mu \hat{D}^{(\mathrm{n})}+\frac{1}{2} \tau\left(L^{2} \hat{P}_{D}+f \hat{\omega}_{z}^{(\mathrm{n})}\right)$ & (DC.2) & $\hat{D}^{(\mathrm{n}+1)}=\hat{D}^{(\mathrm{n})}+\tau\left(f \hat{\omega}_{z}^{(*)}+L^{2} \hat{P}_{C}\right)$ & (DC.7) \\
\hline$\hat{w}^{(*)}=\hat{w}^{(\mathrm{n})}+\frac{1}{2} \tau\left[-\mu\left(\underset{\sim}{i} m+\frac{1}{2 H}\right) \hat{P}_{D}+\hat{B}^{(\mathrm{n})}\right]$ & (DC.3) & $\hat{w}^{(\mathrm{n}+1)}=\hat{w}^{(\mathrm{n})}+\tau\left[-\left(\underset{\sim}{\operatorname{im}}+\frac{1}{2 H}\right) \hat{P}_{C}+\hat{B}^{(*)}\right.$ & (DC.8) \\
\hline$\hat{B}^{(*)}=\hat{B}^{(\mathrm{n})}-\frac{1}{2} \tau N^{2} \hat{w}^{(\mathrm{n})}$ & (DC.4) & $\hat{B}^{(\mathrm{n}+1)}=\hat{B}^{(\mathrm{n})}-\tau N^{2} \hat{w}^{(\mathrm{n}+1)}$ & (DC.9) \\
\hline$\mu \hat{D}^{(*)}+\left(\underset{\sim}{i m}-\frac{1}{2 H}\right) \hat{w}^{(*)}=0$ & (DC.5) & $\hat{D}^{(\mathrm{n}+1)}+\left(\underset{\sim}{i m}-\frac{1}{2 H}\right) \hat{w}^{(\mathrm{n}+1)}=0$ & (DC.10) \\
\hline
\end{tabular}


Scheme l:

We choose

$$
P \equiv P_{D}=P_{C} .
$$

First we use (DC.2), (DC.11) and $\sigma_{f} \equiv 1-\frac{1}{2} \tau^{2} f^{2}$ in (DC.6).

$$
\hat{\omega}_{z}^{(\mathrm{n}+1)}=\sigma_{f} \hat{\boldsymbol{\omega}}_{z}^{(n)}-\mu \tau f \hat{D}^{(n)}-\frac{\tau^{2}}{2} f L^{2} \hat{P} .
$$

Then we use (DC.1), (DC.11) and $\sigma_{f} \equiv 1-\frac{1}{2} \tau^{2} f^{2}$ in (DC.7).

$$
\hat{D}^{(\mathrm{n}+1)}=\sigma_{f} \hat{D}^{(\mathrm{n})}+\mu \tau f \hat{\omega}_{z}^{(\mathrm{n})}+\tau L^{2} \hat{P} .
$$

We use (DC.4), (DC.11) and $\sigma_{N} \equiv 1-\frac{1}{2} \tau^{2} N^{2}$ in (DC.8).

$$
\hat{w}^{(\mathrm{n}+1)}=\sigma_{N} \hat{w}^{(\mathrm{n})}-\tau\left(\underset{\sim}{i m}+\frac{1}{2 H}\right) \hat{P}+\tau \hat{B}^{(\mathrm{n})} .
$$

From (DC.10),

$$
\hat{D}^{(\mathrm{n})}+\left(\underset{\sim}{i} m-\frac{1}{2 H}\right) \hat{w}^{(\mathrm{n})}=0
$$

By using

$$
\hat{\Phi}^{(\mathrm{n}+1)}=e^{-i v \tau} \hat{\Phi}^{(\mathrm{n})}
$$

in (DC.12)-(DC.15) and (DC.9), we write

$$
\left(e^{-i v \tau}-\sigma_{f}\right) \hat{\omega}_{z}=-\mu \tau f \hat{D}-\frac{1}{2} \tau^{2} f L^{2} \hat{P},
$$




$$
\begin{gathered}
\left(e^{-i v \tau}-\sigma_{f}\right) \hat{D}=\tau \mu f \hat{\omega}_{z}+\tau L^{2} \hat{P}, \\
\left(e^{-i v \tau}-\sigma_{N}\right) \hat{w}=-\tau\left({\underset{\sim}{i m}}^{i}+\frac{1}{2 H}\right) \hat{P}+\tau \hat{B}, \\
\left(e^{-i v \tau}-\sigma_{N}\right) \hat{B}=-\tau N^{2} e^{-i v \tau} \hat{w}
\end{gathered}
$$

and

$$
\hat{D}+\left(\underset{\sim}{i} m-\frac{1}{2 H}\right) \hat{w}=0 \text {. }
$$

Eliminate $\hat{\omega}_{z}$ between (DC.17) and (DC.18).

$$
\left[\left(e^{-i v \tau}-\sigma_{f}\right)^{2}+\tau^{2} \mu^{2} f^{2}\right] \hat{D}=\tau\left(e^{-i v \tau}-\sigma_{f}-\mu \frac{1}{2} \tau^{2} f^{2}\right) L^{2} \hat{P}
$$

Eliminate $\hat{w}$ between (DC.19) and (DC.21).

$$
-\left(e^{-i v \tau}-\sigma_{N}\right) \hat{D}=\tau \sigma_{m}^{2} \hat{P}+\tau\left(\underset{\sim}{i} m-\frac{1}{2 H}\right) \hat{B},
$$

where $\sigma_{m}^{2} \equiv m^{2}+1 /\left(4 H^{2}\right)$.

Eliminate $\hat{B}$ between (DC.20), (DC.21) and (DC.23).

$$
-\left[\left(e^{-i v \tau}-\sigma_{N}\right)^{2}+\tau^{2} N^{2} e^{-i v \tau}\right] \hat{D}=\tau\left(e^{-i v \tau}-\sigma_{N}\right) \sigma_{m}^{2} \hat{P}
$$

Eliminate $\hat{P}$ between (DC.22) and (DC.24).

$$
\begin{aligned}
\left(e^{-i v \tau}-\sigma_{N}\right)\left[\left(e^{-i v \tau}-\sigma_{f}\right)^{2}+\mu^{2} \tau^{2} f^{2}\right] \sigma_{m}^{2} \hat{D} & \\
& +\left[\left(e^{-i v \tau}-\sigma_{f}\right)-\mu \frac{1}{2} \tau^{2} f^{2}\right]\left[\left(e^{-i v \tau}-\sigma_{N}\right)^{2}+\tau^{2} N^{2} e^{-i v \tau}\right] L^{2} \hat{D}=0
\end{aligned}
$$

Eliminate $\hat{D}$ between (DC.22) and (DC.24). 


$$
\begin{aligned}
\left(e^{-i v \tau}-\sigma_{N}\right)\left[\left(e^{-i v \tau}-\sigma_{f}\right)^{2}+\mu^{2} \tau^{2} f^{2}\right] \sigma_{m}^{2} \hat{P} \\
+\left[\left(e^{-i v \tau}-\sigma_{N}\right)^{2}+\tau^{2} N^{2} e^{-i v \tau}\right]\left(e^{-i v \tau}-\sigma_{f}-\mu \frac{1}{2} \tau^{2} f^{2}\right) L^{2} \hat{P}=0
\end{aligned}
$$

Both (CD.25a) and (CD.25b) produce third-order equations for the frequency. Their solutions are more challenging than the solution of a second-order equation. We will obtain the frequency for this case through numerical simulations of inertia-gravity modes.

\section{Scheme II:}

We eliminate $\hat{P}_{D}$ and $\hat{w}$ between (DC.2), (DC.3), (DC.5) and DC.10).

$$
\mu\left(L^{2}+\sigma_{m}^{2}\right) \hat{D}^{(*)}=\left(L^{2}+\mu^{2} \sigma_{m}^{2}\right) \hat{D}^{(\mathrm{n})}+\mu \frac{1}{2} \tau f \sigma_{m}^{2} \hat{\omega}_{z}^{(\mathrm{n})}-\frac{1}{2} \tau\left(\underset{\sim}{\underset{i}{m}}-\frac{1}{2 H}\right) L^{2} \hat{B}^{(\mathrm{n})} .
$$

Now we eliminate $\hat{w}$ between (DC.4) and (DC.10).

$$
\left(\underset{\sim}{i m}-\frac{1}{2 H}\right) \hat{B}^{(*)}=\left(\underset{\sim}{i m}-\frac{1}{2 H}\right) \hat{B}^{(\mathrm{n})}+\frac{1}{2} \tau N^{2} \hat{D}^{(\mathrm{n})} .
$$

The equations of the predictor step on the D-grid consist of (DC.1), (DC.26) and (DC.27).

We eliminate $\hat{P}_{C}, \hat{w}^{(\mathrm{n})}$ and $\hat{w}^{(\mathrm{n}+1)}$ between (DC.7), (DC.8) and (DC.10).

$$
\left(L^{2}+\sigma_{m}^{2}\right)\left(\hat{D}^{(\mathrm{n}+1)}-\hat{D}^{(\mathrm{n})}\right)=\tau f \sigma_{m}^{2} \hat{\omega}_{z}^{(*)}-\tau\left(\underset{\sim}{i} m-\frac{1}{2 H}\right) L^{2} \hat{B}^{(*)}
$$

Equations (DC.6), (DC.9) and (DC.28) are the equations of the correction step on the C-grid.

Now we use (DC.28) in (DC.6) to eliminate $\hat{D}^{(*)}$.

$$
\mu\left(L^{2}+\sigma_{m}^{2}\right)\left(\hat{\omega}_{z}^{(\mathrm{n}+1)}-\hat{\omega}_{z}^{(\mathrm{n})}\right)+\mu \frac{1}{2} \tau^{2} f^{2} \sigma_{m}^{2} \hat{\omega}_{z}^{(\mathrm{n})}=-\tau f\left(L^{2}+\mu^{2} \sigma_{m}^{2}\right) \hat{D}^{(\mathrm{n})}+\frac{1}{2} \tau^{2} f\left({\underset{\sim}{i}}^{i}-\frac{1}{2 H}\right) L^{2} \hat{B}^{(\mathrm{n})} .
$$

By using (DC.1) and (DC.27) in (DC.28), we obtain 


$$
\left(L^{2}+\sigma_{m}^{2}\right)\left(\hat{D}^{(\mathrm{n}+1)}-\hat{D}^{(\mathrm{n})}\right)+\frac{1}{2} \tau^{2}\left(f^{2} \sigma_{m}^{2}+N^{2} L^{2}\right) \hat{D}^{(\mathrm{n})}=\mu \tau f \sigma_{m}^{2} \hat{\omega}_{z}^{(\mathrm{n})}-\tau\left(\underset{\sim}{i m}-\frac{1}{2 H}\right) L^{2} \hat{B}^{(\mathrm{n})}
$$

Now we use (DC.10) in (DC.9).

$$
\left(\underset{\sim}{i m}-\frac{1}{2 H}\right)\left(\hat{B}^{(\mathrm{n}+1)}-\hat{B}^{(\mathrm{n})}\right)=\tau N^{2} \hat{D}^{(\mathrm{n}+1)}
$$

By using (DC.16) in (DC.29)-(DC.31), we write

$$
\begin{gathered}
{\left[\mu\left(L^{2}+\sigma_{m}^{2}\right)\left(e^{i v \tau}-1\right)+\mu \frac{1}{2} \tau^{2} f^{2} \sigma_{m}^{2}\right] \hat{\omega}_{z}=-\tau f\left(L^{2}+\mu^{2} \sigma_{m}^{2}\right) \hat{D}+\frac{1}{2} \tau^{2} f L^{2}\left(\underset{\sim}{i m}-\frac{1}{2 H}\right) \hat{B}} \\
{\left[\left(L^{2}+\sigma_{m}^{2}\right)\left(e^{i v \tau}-1\right)+\frac{1}{2} \tau^{2}\left(N^{2} L^{2}+f^{2} \sigma_{m}^{2}\right)\right] \hat{D}=\mu \tau f \sigma_{m}^{2} \hat{\omega}_{z}-\tau L^{2}\left(\underset{\sim}{i} m-\frac{1}{2 H}\right) \hat{B}} \\
\left(e^{i v \tau}-1\right)\left(\underset{\sim}{i} m-\frac{1}{2 H}\right) \hat{B}=\tau N^{2} e^{i v \tau} \hat{D}
\end{gathered}
$$

By eliminating $\hat{\omega}_{z}$ between (DC.32) and (DC.33), we write

$$
\begin{aligned}
& \left\{\left[\left(L^{2}+\sigma_{m}^{2}\right)\left(e^{i v \tau}-1\right)+\frac{1}{2} \tau^{2} f^{2} \sigma_{m}^{2}\right]\left[\left(L^{2}+\sigma_{m}^{2}\right)\left(e^{i v \tau}-1\right)+\frac{1}{2} \tau^{2}\left(N^{2} L^{2}+f^{2} \sigma_{m}^{2}\right)\right]+\tau^{2} f^{2} \sigma_{m}^{2}\left(L^{2}+\mu^{2} \sigma_{m}^{2}\right)\right\} \hat{D}= \\
& -\tau\left(L^{2}+\sigma_{m}^{2}\right)\left(e^{i v \tau}-1\right)\left(\underset{\sim}{i m}-\frac{1}{2 H}\right) L^{2} \hat{B}
\end{aligned}
$$

(DC.35)

By eliminating $\hat{B}$ between (DC.34) and (DC.35), we write

$$
\begin{array}{r}
\left\{\left[\left(L^{2}+\sigma_{m}^{2}\right)\left(e^{i v \tau}-1\right)+\frac{1}{2} \tau^{2} f^{2} \sigma_{m}^{2}\right]\left[\left(L^{2}+\sigma_{m}^{2}\right)\left(e^{i v \tau}-1\right)+\frac{1}{2} \tau^{2}\left(N^{2} L^{2}+f^{2} \sigma_{m}^{2}\right)\right]+\tau^{2} f^{2} \sigma_{m}^{2}\left(L^{2}+\mu^{2} \sigma_{m}^{2}\right)\right\} \hat{D}= \\
-\tau^{2}\left(L^{2}+\sigma_{m}^{2}\right) N^{2} L^{2} e^{i v \tau} \hat{D}
\end{array}
$$

(DC.38) 
After some manipulations, we rewrite (DC.38) as

$$
\begin{aligned}
& \left(L^{2}+\sigma_{m}^{2}\right)^{2}\left(e^{-i v \tau}-1\right)^{2} \\
& +\left[\frac{1}{2} \tau^{2}\left(N^{2} L^{2}+f^{2} \sigma_{m}^{2}\right)+\frac{1}{2} \tau^{2} f^{2} \sigma_{m}^{2}\right]\left(L^{2}+\sigma_{m}^{2}\right)\left(e^{-i v \tau}-1\right)+\tau^{2} L^{2}\left(L^{2}+\sigma_{m}^{2}\right) N^{2} e^{-i v \tau} \\
& +\frac{1}{4} \tau^{4} f^{2} \sigma_{m}^{2}\left(N^{2} L^{2}+f^{2} \sigma_{m}^{2}\right)+\tau^{2} f^{2} \sigma_{m}^{2}\left(L^{2}+\mu^{2} \sigma_{m}^{2}\right)=0
\end{aligned}
$$

and then

$$
\begin{aligned}
& e^{-2 i v \tau}-\left[2-\frac{1}{2} \tau^{2}\left(\frac{N^{2} L^{2}+f^{2} \sigma_{m}^{2}}{L^{2}+\sigma_{m}^{2}}\right)-\frac{1}{2} \tau^{2}\left(\frac{f^{2} \sigma_{m}^{2}+2 L^{2} N^{2}}{L^{2}+\sigma_{m}^{2}}\right)\right] e^{-i v \tau} \\
& +\left[1+\left(\frac{\tau^{2} f^{2} \sigma_{m}^{2}}{L^{2}+\sigma_{m}^{2}}\right)\left(\frac{L^{2}+\mu^{2} \sigma_{m}^{2}}{L^{2}+\sigma_{m}^{2}}\right)-\frac{1}{2} \tau^{2}\left(\frac{N^{2} L^{2}+f^{2} \sigma_{m}^{2}}{L^{2}+\sigma_{m}^{2}}\right)-\frac{\frac{1}{2} \tau^{2} f^{2} \sigma_{m}^{2}}{L^{2}+\sigma_{m}^{2}}+\left(\frac{\frac{1}{4} \tau^{4} f^{2} \sigma_{m}^{2}}{L^{2}+\sigma_{m}^{2}}\right)\left(\frac{N^{2} L^{2}+f^{2} \sigma_{m}^{2}}{L^{2}+\sigma_{m}^{2}}\right)\right]=0
\end{aligned}
$$

(DC.40)

Real and imaginary parts of this equation produce

$$
\begin{aligned}
& e^{2 v_{i} \tau} \cos \left(2 v_{r} \tau\right)-e^{v_{i} \tau}\left[2-\frac{\frac{1}{2} \tau^{2} f^{2} \sigma_{m}^{2}+\tau^{2} L^{2} N^{2}}{L^{2}+\sigma_{m}^{2}}-\frac{1}{2} \tau^{2}\left(\frac{N^{2} L^{2}+f^{2} \sigma_{m}^{2}}{L^{2}+\sigma_{m}^{2}}\right)\right] \cos \left(v_{r} \tau\right)+ \\
& +\left[1+\left(\frac{\tau^{2} f^{2} \sigma_{m}^{2}}{L^{2}+\sigma_{m}^{2}}\right)\left(\frac{L^{2}+\mu^{2} \sigma_{m}^{2}}{L^{2}+\sigma_{m}^{2}}\right)-\frac{1}{2} \tau^{2}\left(\frac{N^{2} L^{2}+f^{2} \sigma_{m}^{2}}{L^{2}+\sigma_{m}^{2}}\right)-\frac{\frac{1}{2} \tau^{2} f^{2} \sigma_{m}^{2}}{L^{2}+\sigma_{m}^{2}}+\left(\frac{\frac{1}{4} \tau^{4} f^{2} \sigma_{m}^{2}}{L^{2}+\sigma_{m}^{2}}\right)\left(\frac{N^{2} L^{2}+f^{2} \sigma_{m}^{2}}{L^{2}+\sigma_{m}^{2}}\right)\right]=0
\end{aligned}
$$

(DC.41a)

and

$$
e^{v_{i} \tau}=\frac{\sin \left(v_{r} \tau\right)}{\sin \left(2 v_{r} \tau\right)}\left[2-\frac{\frac{1}{2} \tau^{2} f^{2} \sigma_{m}^{2}+\tau^{2} L^{2} N^{2}}{L^{2}+\sigma_{m}^{2}}-\frac{1}{2} \tau^{2}\left(\frac{N^{2} L^{2}+f^{2} \sigma_{m}^{2}}{L^{2}+\sigma_{m}^{2}}\right)\right] .
$$


The solution of (DC.41a) and (DC.41b) is shown in the figure. 
(a)

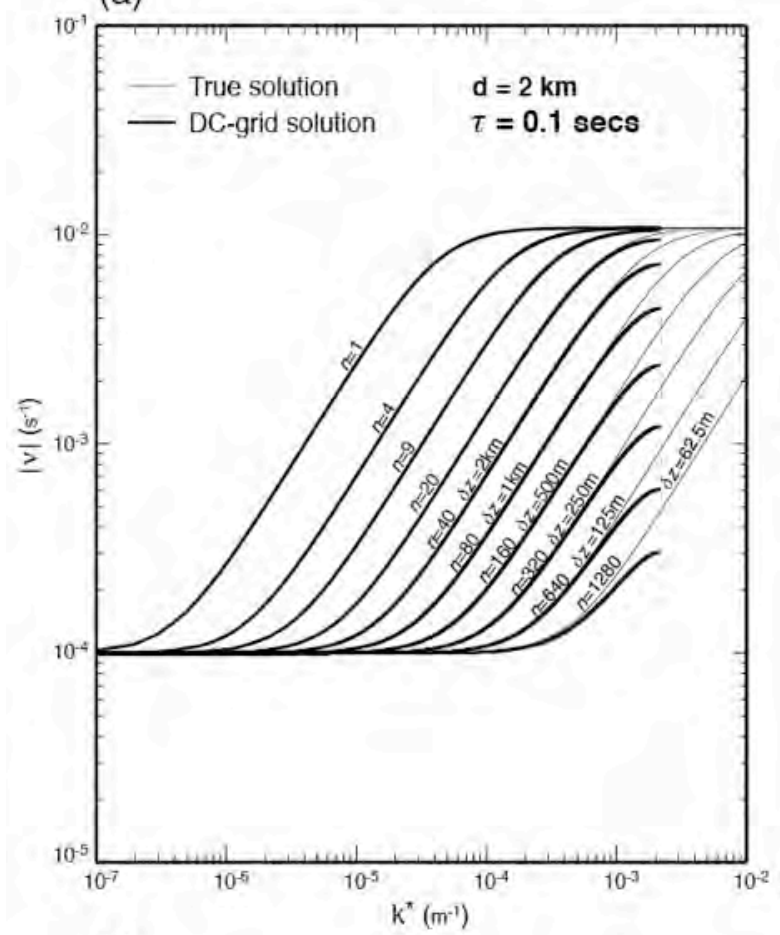

(c)

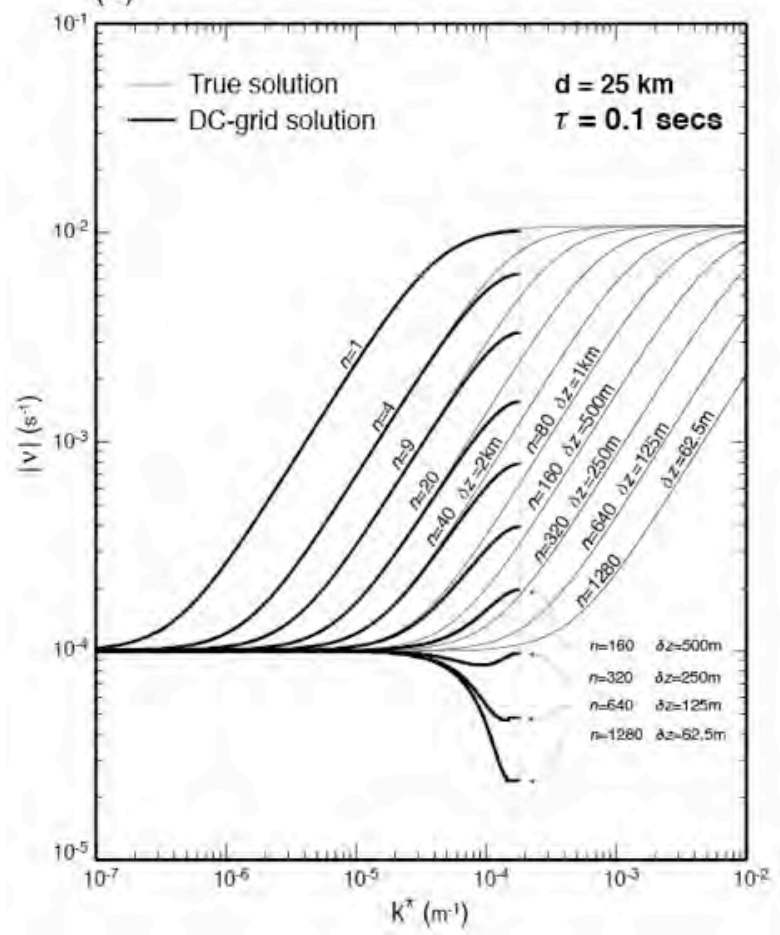

(b)

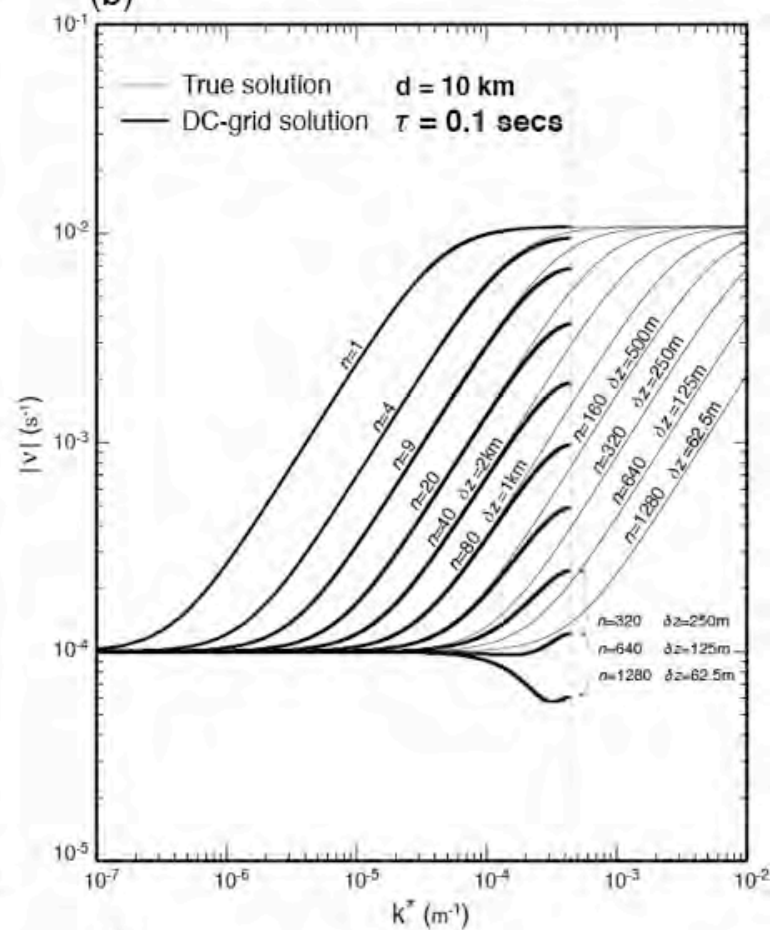

(d)

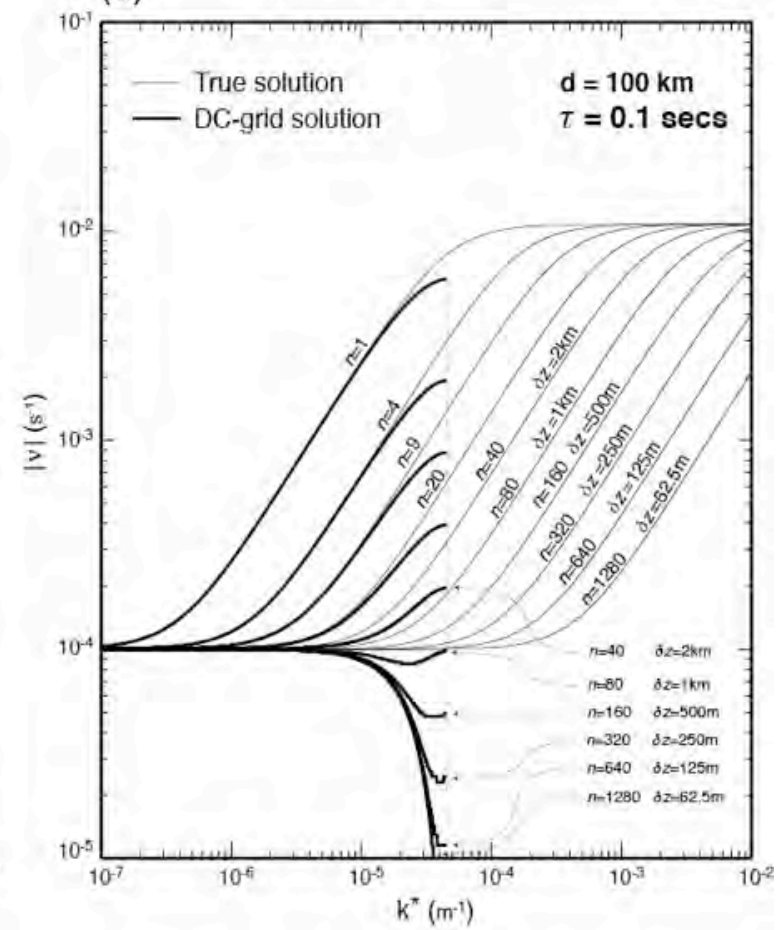

Frequency of inertia-gravity modes on the DC-grid using scheme II. 
Numerical model results (Inertia-gravity modes):

A numerical model inspired by the scheme II discussed above is constructed on the DCgrid.

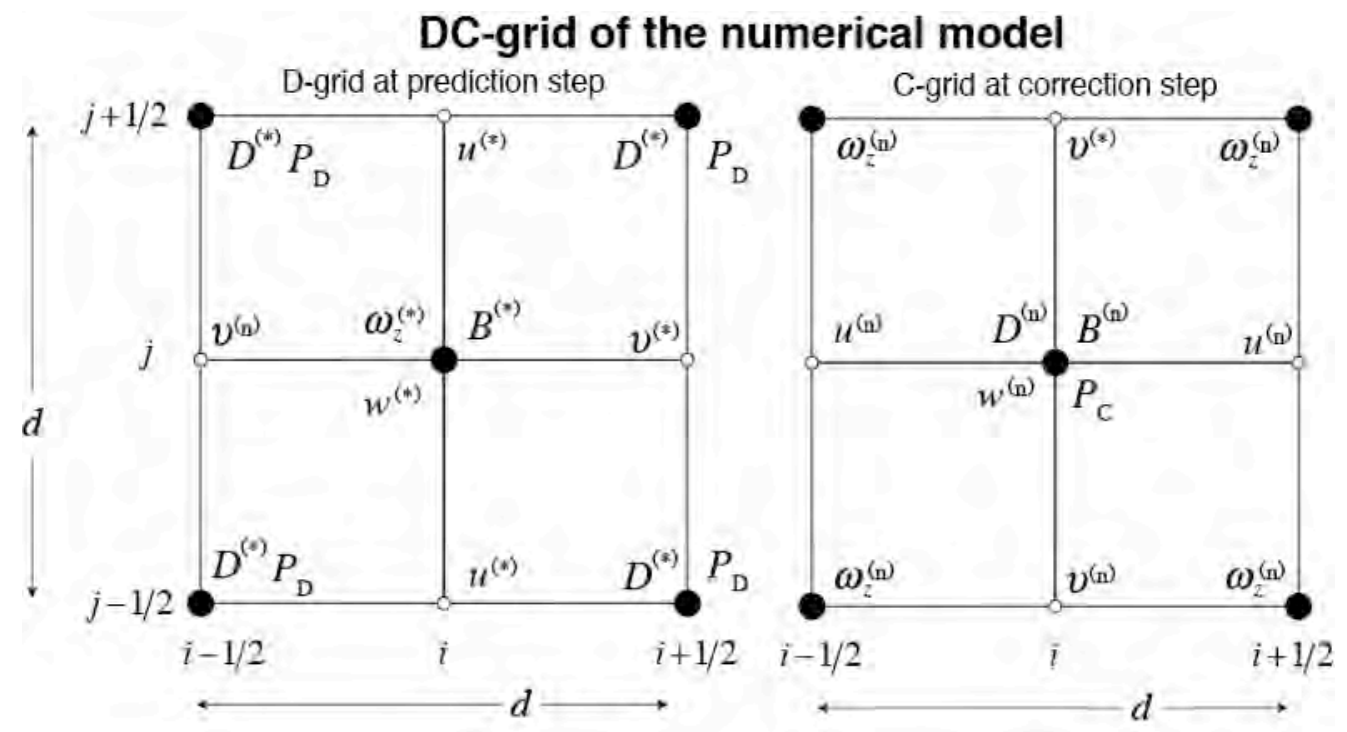

The equations of the system are given by 


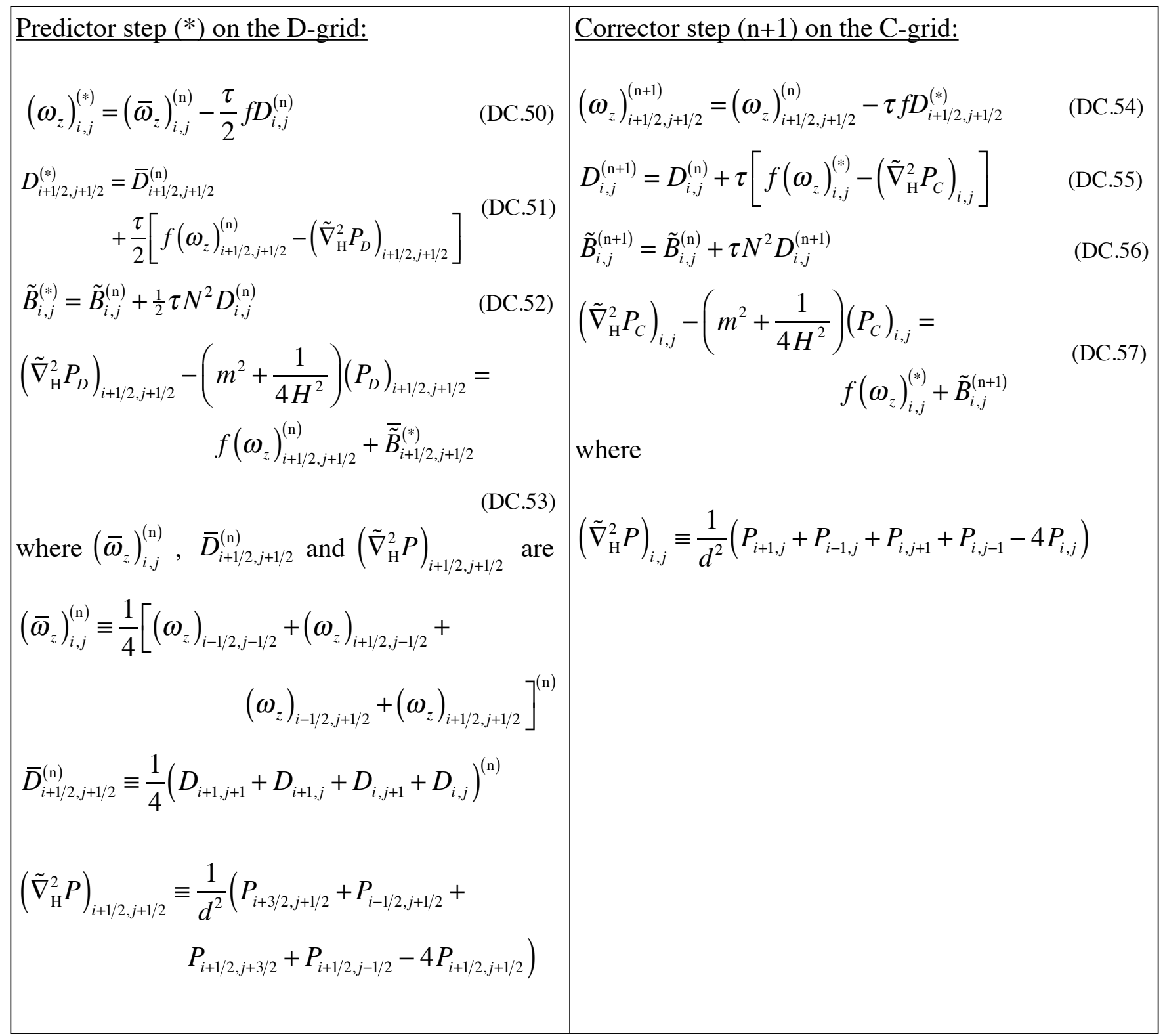

We made the same simulations described in "Numerical integrations" section with the DC-grid model, which uses Eqs. (DC-50)-(DC.57). We tabulate the results below. 


\begin{tabular}{|c|c|c|c|c|c|c|c|c|}
\hline \multirow{3}{*}{$\begin{array}{l}\text { Horizontal } \\
\text { scale } \\
\text { of } \\
\text { perturbation }\end{array}$} & \multirow{3}{*}{$\begin{array}{c}\text { Vertical } \\
\text { wavenumber } \\
\text { (Vertically } \\
\text { continuous } \\
\text { solutions) }\end{array}$} & \multirow{2}{*}{$\begin{array}{c}\text { True frequency } v \\
\text { (analytic) } \\
\left(10^{-1} s^{-1}\right)\end{array}$} & \multirow{3}{*}{$\begin{array}{c}\text { Horizontal } \\
\text { grid } \\
\text { distance } \\
\text { (d) }\end{array}$} & \multicolumn{5}{|c|}{ Numerical frequency $v\left(10^{-4} s^{-1}\right)$} \\
\hline & & & & Z-grid & C-grid & CD-grid & D-grid & DC-grid \\
\hline & & $\tilde{B} \quad D \quad \omega_{z}$ & & $\tilde{B} \quad D \quad \omega_{z}$ & $\tilde{B} \quad D \quad \omega_{z}$ & $\tilde{B} \quad D \quad \omega_{z}$ & $\tilde{B} \quad D \quad \omega_{z}$ & $\tilde{B} \quad D$ \\
\hline \multirow{3}{*}{$\begin{array}{l}L=4 k m \\
k=\frac{\pi}{2 k m}\end{array}$} & $\begin{array}{l}n=320 \\
\delta=250 \mathrm{~m}\end{array}$ & 18.84724224 & \multirow{3}{*}{$50 \mathrm{~m}$} & 18.84578676 & 18.84578676 & 18.81193205 & $18+81193205$ & 18.8344883309 \\
\hline & $\begin{array}{r}n=640 \\
\delta z=125 \mathrm{~m}\end{array}$ & 9.57153193 & & 9.56927399 & 9.56927399 & 9.55472218 & 9.55472218 & 9.5663600901 \\
\hline & $\begin{array}{c}n=1280 \\
\delta z=62.5 \mathrm{~m}\end{array}$ & 4.87709384 & & 4.87597804 & 4.87522137 & 4.86842190 & 4.86842190 & 4.8752213743 \\
\hline$L=20 \mathrm{~km}$ & $\begin{array}{l}n=160 \\
\delta z=500 \mathrm{~m}\end{array}$ & 7.69121581 & \multirow{3}{*}{$250 \mathrm{~m}$} & 7.68867511 & 7.68867511 & 7.67740140 & 7.67740140 & 7.6886751189 \\
\hline \multirow{2}{*}{$k=\frac{\pi}{10 \mathrm{~km}}$} & $\begin{array}{l}n=320 \\
\delta z=250 m\end{array}$ & 3.94883169 & & 3,94771632 & 3.94771632 & 3.94177246 & 3.94177246 & 3.9472203211 \\
\hline & $\begin{array}{l}n=640 \\
d z=125 \mathrm{~m}\end{array}$ & 2.15679036 & & 2.15635435 & 2.15561455 & 2.15310304 & 2.15310304 & 2.1556145558 \\
\hline \multirow{2}{*}{$L=50 \mathrm{~km}$} & $\begin{array}{l}n=80 \\
\delta z=1 \mathrm{~km}\end{array}$ & 6.18745719 & & 6.18545511 & 6.18423750 & 6.17572764 & 6.17572764 & 6.1854551163 \\
\hline & $\begin{array}{l}n=160 \\
\delta z=500 m\end{array}$ & 3.21618641 & $625 \mathrm{~m}$ & 3.21555031 & 3.21489219 & 3.21062100 & 3.21062100 & 3.2148921957 \\
\hline$k=\frac{\pi}{25 k m}$ & $\begin{array}{l}n=320 \\
\delta z=250 m\end{array}$ & 1.82684828 & & 1.82650735 & 1.82565821 & 1.82364465 & 1.82364465 & 1.8257643131 \\
\hline & $\begin{array}{l}n=640 \\
\delta z=125 m\end{array}$ & 1.25874243 & & 1.25860047 & 1.25739149 & 1.25668732 & 1.25668732 & 1.2579453246 \\
\hline$L=300 \mathrm{~km}$ & $\begin{array}{l}n=80 \\
\delta z=1 \mathrm{~km}\end{array}$ & 1.82682191 & & 1,82650735 & 1.82565821 & 1.82364465 & 1.82364465 & 1.8257643131 \\
\hline$k=\frac{\pi}{2}$ & $\begin{array}{l}n=160 \\
\delta=500 \mathrm{~m}\end{array}$ & 1.25874064 & $2.5 \mathrm{~km}$ & 1.25860047 & 1.25739149 & 1.25668732 & 1.25668732 & 1.2579453246 \\
\hline $100 \mathrm{~km}$ & $\begin{array}{l}n=320 \\
\delta z=250 m\end{array}$ & 1.07056681 & & 1.07053521 & 1.06907801 & 1.06885977 & 1.06885977 & 1.0700247458 \\
\hline
\end{tabular}

Table DC.1b. Same as Table 1b in page (Numerical integrations page 12), except that DC-grid results are added to this table. 


\begin{tabular}{|c|c|c|c|c|c|c|c|c|}
\hline \multirow{3}{*}{$\begin{array}{c}\text { Horizontal } \\
\text { scale } \\
\text { of } \\
\text { perturbation }\end{array}$} & \multirow{3}{*}{\begin{tabular}{|c|} 
Vertical \\
wavenumber \\
(Vertically \\
continuous \\
solutions)
\end{tabular}} & \multirow{2}{*}{$\begin{array}{c}\text { True frequency } v \\
\text { (analytic) } \\
\left(10^{-4} s^{-1}\right)\end{array}$} & \multirow{3}{*}{$\begin{array}{l}\text { Horizontal } \\
\text { grid } \\
\text { distance } \\
\text { (d) }\end{array}$} & \multicolumn{5}{|c|}{ Numerical frequency $v\left(10^{-4} s^{-1}\right)$} \\
\hline & & & & Z-grid & C-grid & CD-grid & D-grid & DC-grid \\
\hline & & $\tilde{B} \quad D \quad \omega_{\tau}$ & & $\tilde{B} \quad D \quad \omega_{z}$ & $\tilde{B} \quad D \quad \omega_{z}$ & $\tilde{B} \quad D \quad \omega_{z}$ & $\tilde{B} \quad D \quad \omega_{z}$ & $\tilde{B} \quad D \quad \omega_{z}$ \\
\hline \multirow{3}{*}{$\begin{array}{l}L=4 k m \\
k=\frac{\pi}{2 k m}\end{array}$} & $\begin{array}{l}n=320 \\
\delta z=250 m\end{array}$ & 18.84724224 & \multirow{3}{*}{$1 \mathrm{~km}$} & 17.01837840 & 16.99996024 & 8.51149459 & 8.51149459 & 16.9999602467 \\
\hline & $\begin{array}{r}n=640 \\
\delta z=125 \mathrm{~m}\end{array}$ & 9.57153193 & & 8.63549382 & 8.59062798 & 4.31715357 & 4.31715357 & 8.5906279836 \\
\hline & $\begin{array}{l}n=1280 \\
\delta z=62.5 \mathrm{~m}\end{array}$ & 4.87709384 & & 4.41296903 & 4.32726260 & 2.20663949 & 2.20663949 & 4.3278587320 \\
\hline \multirow{3}{*}{$\begin{array}{l}L=20 \mathrm{~km} \\
k=\frac{\pi}{10 \mathrm{~km}}\end{array}$} & $\begin{array}{l}n=160 \\
\delta z=500 m\end{array}$ & 7.69121581 & \multirow{3}{*}{$5 \mathrm{~km}$} & 6.94121222 & 6.88794705 & 3.47098956 & 3.47060611 & 6.8864372065 \\
\hline & $\begin{array}{l}n=320 \\
\delta z=250 m\end{array}$ & 3.94883169 & & 3.58220370 & 3.47598213 & 1.79110185 & 1.79110185 & 3.4763667739 \\
\hline & $\begin{array}{l}n=640 \\
\partial z=-125 \mathrm{~m}\end{array}$ & 2.15679036 & & 1.98998711 & 1.79171475 & 0.99499355 & 0.99499355 & 1.7963249206 \\
\hline \multirow{3}{*}{$L=50 \mathrm{kn}$} & $\begin{array}{l}n=80 \\
\delta z=1 \mathrm{~km}\end{array}$ & 6.18745719 & \multirow{4}{*}{$-12.5 \mathrm{~km}$} & 5.58902802 & 5.52222298 & 2.79451401 & 2.79476261 & 5.5212524668 \\
\hline & $\begin{array}{c}n=160 \\
\delta z=500 m\end{array}$ & 3.21618041 & & 2.92840478 & 2.79750013 & 1.46420239 & 1.46413415 & 2.7984969300 \\
\hline & $\begin{array}{c}n=320 \\
\delta z=256 m\end{array}$ & 1.82684828 & & 1.70137701 & 1.46454368 & 0.85068850 & 0.85068850 & 1.4732661103 \\
\hline$k=\frac{\pi}{25 \mathrm{~km}}$ & $\begin{array}{l}n=640 \\
\delta z=125 \mathrm{~m}\end{array}$ & 1.25874243 & & 1.21395442 & 0.85073457 & 0.60701239 & ๑. $6 \emptyset 698893$ & 0.9013837126 \\
\hline \multirow{3}{*}{$\begin{array}{l}L=200 \mathrm{~km} \\
k=\frac{\pi}{100 \mathrm{~km}}\end{array}$} & $\begin{array}{l}n=80 \\
\delta z=1 \mathrm{~km}\end{array}$ & 1.82682191 & \multirow{3}{*}{$50 \mathrm{~km}$} & 1.70137701 & 1.46447541 & 0.85068850 & 0.85066547 & 1.4732661103 \\
\hline & $\begin{array}{c}n=160 \\
\delta z=500 m\end{array}$ & 1.25874004 & & 1.21395442 & 0.85073457 & 0.60700066 & 0.60698893 & 0.9013837126 \\
\hline & $\begin{array}{l}n=320 \\
\delta z=258 \mathrm{~mm}\end{array}$ & 1.07056681 & & 1.05756165 & 0.60700066 & 0.52879862 & 0.52878082 & 0.7278607696 \\
\hline
\end{tabular}

Table DC.2b. Same as Table 2b in page (Numerical integrations page 16), except that DC-grid results are added to this table. 


\begin{tabular}{|c|c|c|c|c|c|c|c|c|c|c|c|c|}
\hline \multirow{3}{*}{$\begin{array}{c}\text { Horizontal } \\
\text { scale } \\
\text { of } \\
\text { perturbation }\end{array}$} & \multirow{3}{*}{$\begin{array}{c}\text { Vertical } \\
\text { wavenumber } \\
\text { (Vertically } \\
\text { continuous } \\
\text { solutions) }\end{array}$} & \multirow{2}{*}{$\begin{array}{c}\text { True frequency } v \\
\text { (analytic) } \\
\left(10^{-4} s^{-1}\right)\end{array}$} & \multirow{3}{*}{$\begin{array}{l}\text { Horizontal } \\
\text { grid } \\
\text { distance } \\
\text { (d) }\end{array}$} & \multicolumn{9}{|c|}{ Numerical frequency $v\left(10^{-4} s^{-1}\right)$} \\
\hline & & & & Z-grid & \multicolumn{2}{|l|}{ C-grid } & CD-grid & D-grid & A-grid & B-grid & E-grid & DC-grid \\
\hline & & $\tilde{B} \quad D \quad \omega_{z}$ & & $\tilde{B} \quad D \quad \omega_{z}$ & $\tilde{B} \quad D$ & $\omega_{z}$ & $\tilde{B} D \omega_{\varepsilon}$ & $\begin{array}{lll}\tilde{B} & D & \omega_{z}\end{array}$ & $\tilde{B} \quad D \omega_{z}$ & $\tilde{B} D \omega_{z}$ & $\tilde{B} D \omega_{z}$ & $\tilde{B} D \omega_{z}$ \\
\hline \multirow{5}{*}{$\begin{array}{c}L=4 / m n \\
k=\frac{\pi}{2 k m}\end{array}$} & $\begin{array}{l}n=320 \\
\delta z=250 \mathrm{~mm}\end{array}$ & 18.84724224 & \multirow{5}{*}{$\begin{array}{c}2 \mathrm{~km} \\
\text { (For E-grid, } \\
2.82 \mathrm{~km} \text { ) }\end{array}$} & 12.13438645 & 12.09235047 & 0.0 & 0.0 & 0.0 & 0.0 & 0.0 & 0.0 & 12.087697782 \\
\hline & \multirow{3}{*}{$\begin{array}{r}n=640 \\
\delta z=125 m\end{array}$} & \multirow{3}{*}{9.57153193} & & \multirow{3}{*}{6.15636420} & & & 0.0 & 0.0 & 1.0606135 & 1.0660135 & 1.0606135 & \\
\hline & & & & & 6.07423173 & \multirow[t]{2}{*}{$\theta . \theta$} & 0.0 & 0.0 & 0.0 & 0.0 & 0.0 & 6.0742317355 \\
\hline & & & & & & & 0.0 & 0.0 & 1.0000135 & 1.0000135 & 1.0000135 & 0.0 \\
\hline & $\begin{array}{l}n=1280 \\
\delta z=62.5 \mathrm{~mm}\end{array}$ & 4.87709384 & & 3.20080759 & $\begin{array}{c}3.04064329 \\
0.0\end{array}$ & 0.0 & $\begin{array}{l}0.0 \\
0.0\end{array}$ & $\begin{array}{l}0.0 \\
0.0\end{array}$ & $\begin{array}{c}0.0 \\
1.0000135\end{array}$ & $\begin{array}{c}0.0 \\
1.0000135\end{array}$ & $\begin{array}{c}0.0 \\
1.0000135\end{array}$ & $\begin{array}{c}3.0406432962 \\
0.0\end{array}$ \\
\hline \multirow{4}{*}{$\begin{array}{c}L=20 \mathrm{~km} \\
k=\frac{\pi}{10 \mathrm{~km}}\end{array}$} & $n=160$ & \multirow[t]{2}{*}{7.69121581} & \multirow{4}{*}{$\begin{array}{l}10 \mathrm{~km} \\
\text { (For E-grid, } \\
14.14 \mathrm{~km} \text { ) }\end{array}$} & \multirow[t]{2}{*}{4.96380574} & 4.86239383 & \multirow[t]{2}{*}{0.0} & 0.0 & 0.0 & 0.0 & 0.0 & 0.0 & 4.8616413705 \\
\hline & $\delta z=560 \mathrm{~m}$ & & & & 0.0 & & 0.0 & 0.0 & 1.0000135 & 1.0000135 & 1.0000135 & 0.0 \\
\hline & $\begin{array}{l}n=320 \\
\delta z=250 m\end{array}$ & 3.94883169 & & 2.63026846 & $\begin{array}{c}2.43289139 \\
0.0\end{array}$ & 0.0 & 0.0 & $\begin{array}{l}0.0 \\
0.0\end{array}$ & $\begin{array}{c}0.0 \\
1.0000135\end{array}$ & $\begin{array}{c}0.0 \\
1.0000135\end{array}$ & $\begin{array}{c}0.0 \\
1.0000135\end{array}$ & $\begin{array}{c}2.4328913913 \\
0.0\end{array}$ \\
\hline & $\begin{array}{l}n=640 \\
\delta z=125 \mathrm{~m}\end{array}$ & 2.15679036 & & 1.57489104 & $\begin{array}{r}1.21672837 \\
0.0\end{array}$ & 0.8 & $\begin{array}{l}0.0 \\
0.0\end{array}$ & $\begin{array}{l}0.0 \\
0.0\end{array}$ & $\begin{array}{c}0.0 \\
1.0000135\end{array}$ & $\begin{array}{c}0.0 \\
1.0000135\end{array}$ & $\begin{array}{c}0.0 \\
1.0000135\end{array}$ & $\begin{array}{c}1.2166812492 \\
0.0\end{array}$ \\
\hline & $\begin{array}{l}n=80 \\
\delta z=1 \mathrm{~km}\end{array}$ & 6.18745719 & & 4.01738191 & $\begin{array}{r}3.89099907 \\
0.0\end{array}$ & 0.0 & $\begin{array}{l}0.0 \\
0.0\end{array}$ & $\begin{array}{l}0.0 \\
0.0\end{array}$ & $\begin{array}{c}0.0 \\
1.0000135\end{array}$ & $\begin{array}{c}0.0 \\
1.0000135\end{array}$ & $\begin{array}{c}0.0 \\
1.0000135\end{array}$ & $\begin{array}{c}3.8909990755 \\
0.0\end{array}$ \\
\hline & $\begin{array}{l}n=160 \\
\delta z=500 m\end{array}$ & 3.21618041 & $25 \mathrm{~km}$ & 2.18834818 & $\begin{array}{c}1.94658445 \\
0.0\end{array}$ & 0,0 & 0.0 & 0.0 & $\begin{array}{c}0.0 \\
1.0000135\end{array}$ & $\begin{array}{c}0.0 \\
1.0000135\end{array}$ & $\begin{array}{c}0.0 \\
1.0000135\end{array}$ & $\begin{array}{c}1.9464638498 \\
0.0\end{array}$ \\
\hline$k=\frac{\pi}{25 \mathrm{~km}}$ & $n=320$ & 1.82684828 & $35.35 \mathrm{~km}$ ) & 1.39551912 & 0.97338269 & 0.0 & 0.0 & 0.0 & 0.0 & 0.0 & 0.0 & 0.9733826967 \\
\hline & $\delta z=250 \mathrm{~m}$ & & & & 0.0 & & 0.0 & 0.0 & 1.0000135 & 1.0000135 & 1.0000135 & 0.0 \\
\hline & $\begin{array}{l}n=640 \\
\delta z=125 \mathrm{~m}\end{array}$ & 1.25874243 & & 1.11214693 & $\begin{array}{c}0.48671396 \\
0.0\end{array}$ & 0.0 & $\begin{array}{l}0.0 \\
0.0\end{array}$ & $\begin{array}{l}0.0 \\
0.0\end{array}$ & $\begin{array}{c}0.0 \\
1.0000135\end{array}$ & $\begin{array}{c}0.0 \\
1.0000135\end{array}$ & $\begin{array}{c}0.0 \\
1.0000135\end{array}$ & $\begin{array}{c}0.4867064283 \\
0.0\end{array}$ \\
\hline & $n=80$ & 1.82682191 & & 1.39545713 & $\emptyset .97338269$ & 0.0 & 0.0 & 0.0 & 0.0 & 0.0 & 0.0 & 0.9733525386 \\
\hline & $\delta z=1 \mathrm{~km}$ & & & & 0.0 & & 0.0 & 0.0 & 1.0000135 & 1.0000135 & 1.0000135 & 0.0 \\
\hline $\begin{array}{c}L=200 \mathrm{~km} \\
\pi\end{array}$ & $\begin{array}{l}n=160 \\
\delta z=590 m\end{array}$ & 1.25874004 & $\begin{array}{l}100 \mathrm{~km} \\
\text { (For E-grid, }\end{array}$ & 1.11214693 & $\begin{array}{c}0.48670642 \\
0.0\end{array}$ & 9.0 & $\begin{array}{l}0.0 \\
0.0\end{array}$ & $\begin{array}{l}0.0 \\
0.0\end{array}$ & $\begin{array}{c}0.0 \\
1.0000135\end{array}$ & $\begin{array}{c}0.0 \\
1.0000135\end{array}$ & $\begin{array}{c}0.0 \\
1.0000135\end{array}$ & $\begin{array}{c}0.486706428= \\
0.0\end{array}$ \\
\hline $100 \mathrm{~km}$ & $\begin{array}{l}n=320 \\
\delta z=25 \mathrm{~mm}\end{array}$ & 1. 07056681 & & 1.02918678 & $\begin{array}{c}0.24335698 \\
0.0\end{array}$ & 0.0 & $\begin{array}{l}0.0 \\
0.0\end{array}$ & $\begin{array}{l}0.0 \\
0.0\end{array}$ & $\begin{array}{c}0.0 \\
1.0000135\end{array}$ & $\begin{array}{c}0.0 \\
1.0000135\end{array}$ & $\begin{array}{c}0.0 \\
1.0000135\end{array}$ & $\begin{array}{c}0.2433569843 \\
0.0\end{array}$ \\
\hline
\end{tabular}

Table DC.3. Same as Table 3 in page (Numerical integrations page 17), except that DC-grid results are added to this table.

The DC-grid frequencies are virtually identical to the C-grid frequencies in these three tables. One exception is that, with the smallest resolvable horizontal grid distance, the vorticity is coupled to the divergence and buoyancy perturbation with the DC-grid (Table DC.3 far right column) unlike the C-grid, on which the vorticity is not. When the perturbation is given to the vorticity, the divergence and buoyancy cannot recognize vorticity perturbation on both the DCand C-grids. 
Normal mode analysis for the DC-grid (Rossby modes):

\begin{tabular}{|c|c|c|c|}
\hline \multicolumn{2}{|l|}{ Predictor step $(*)$ on the D-grid: } & \multicolumn{2}{|l|}{ Corrector step $(\mathrm{n}+1)$ on the C-grid: } \\
\hline$\hat{\omega}_{z}^{(*)}=\mu \hat{\omega}_{z}^{(n)}-\frac{\tau}{2} f_{0} \hat{D}_{D}-\underset{i}{i} \frac{\tau}{2} \frac{\beta}{f_{0}} \tilde{\xi}_{k} \hat{P}_{D}$ & (DC.60) & $\hat{\omega}_{z}^{(\mathrm{n}+1)}=\hat{\omega}_{z}^{(\mathrm{n})}-\mu \tau f_{0} \hat{D}_{C}-i \mu \tau \tau \frac{\beta}{f_{0}} \tilde{\xi}_{k} \hat{P}_{C}$ & (DC.65) \\
\hline $0=\mu L^{2} \hat{P}_{D}+f \hat{\omega}_{z}^{(\mathrm{n})}$ & (DC.61) & $0=f_{0} \hat{\omega}_{z}^{(*)}+L^{2} \hat{P}_{C}$ & (DC.66) \\
\hline $0=-\left(\underset{\sim}{i m}+\frac{1}{2 H}\right) \hat{P}_{D}+\hat{B}^{(\mathrm{n})}$ & (DC.62) & $0=-\left({\underset{\sim}{i}}_{i}+\frac{1}{2 H}\right) \hat{P}_{C}+\hat{B}^{(*)}$ & (DC.67) \\
\hline$\hat{B}^{(*)}=\hat{B}^{(\mathrm{n})}-\frac{1}{2} \tau N^{2} \hat{w}_{D}$ & (DC.63) & $\hat{B}^{(\mathrm{n}+1)}=\hat{B}^{(\mathrm{n})}-\tau N^{2} \hat{w}_{C}$ & (DC.68) \\
\hline$\mu \hat{D}_{D}+\left(\underset{\sim}{i m}-\frac{1}{2 H}\right) \hat{w}_{D}=0$ & (DC.64) & $\hat{D}_{C}+\left(\underset{\sim}{i m}-\frac{1}{2 H}\right) \hat{w}_{C}=0$ & (DC.69) \\
\hline
\end{tabular}

\section{Modified system:}

Since the divergence and vertical velocity are diagnostic variables in the quasigeostrophic (with quasi-static approximation), the system contains more equations than needed. To find a unique solution, we ignore the equations (DC.60)-(DC.64) at the predictor step. (See pages 51-53 of CD-grid section.) Then the equations (DC.65)-(DC.69) are modified as follows:

$$
\begin{gathered}
\hat{\omega}_{z}^{(\mathrm{n}+1)}=\hat{\omega}_{z}^{(\mathrm{n})}-\mu \tau f_{0} \hat{D}_{C}-i \sim \mu \tau \frac{\beta}{f_{0}} \tilde{\xi}_{k} \hat{P}_{C} \\
0=f_{0} \hat{\omega}_{z}^{(\mathrm{n}+1)}+L^{2} \hat{P}_{C} \\
0=-\left(\underset{\sim}{i} m+\frac{1}{2 H}\right) \hat{P}_{C}+\hat{B}^{(\mathrm{n}+1)} \\
\hat{B}^{(\mathrm{n}+1)}=\hat{B}^{(\mathrm{n})}-\tau N^{2} \hat{w}_{C}
\end{gathered}
$$




$$
\hat{D}_{C}+\left(\underset{\sim}{i m}-\frac{1}{2 H}\right) \hat{w}_{C}=0
$$

Then, by eliminating $\hat{w}$ between (DC.73) and (DC.74) and using (DC.17) in all equations, we write

$$
\begin{gathered}
\left(e^{-i v \tau}-1\right) \hat{\omega}_{z}=-\mu \tau f_{0} \hat{D}-\underset{\sim}{i} \mu \tau \frac{\beta}{f_{0}} \tilde{\xi}_{k} \hat{P} \\
\mu f_{0} e^{-i v \tau} \hat{\omega}_{z}=-L^{2} \hat{P} \\
\left(e^{-i v \tau}-1\right)\left(\underset{\sim}{i} m-\frac{1}{2 H}\right) \hat{B}=\tau N^{2} \hat{D} \\
0=\left(m^{2}+\frac{1}{4 H^{2}}\right) \hat{P}+\left(\underset{\sim}{i} m-\frac{1}{2 H}\right) e^{-i v \tau} \hat{B}
\end{gathered}
$$

Then we obtain

$$
N^{2}\left[\left(e^{-i v \tau}-1\right) L^{2}-\underset{\sim}{i} \mu^{2} \tau \beta \tilde{\xi} k e^{-i v \tau}\right] \hat{P}=-\mu^{2} f_{0}^{2}\left(e^{-i v \tau}-1\right)\left(m^{2}+\frac{1}{4 H^{2}}\right) \hat{P}
$$

Equation (DC.79) is identical to Eq. (RS1C.2.13, page 54 of CD-grid) except the factor $\mu^{2}$. Therefore, we can follow the same procedure described in pages 51-58 of CD-grid to obtain the dispersion equation for the DC-grid by

$$
\frac{\sin \left(v_{r} \tau\right)}{\cos \left(v_{r} \tau\right)}=\frac{-\tau \mu^{2} \beta \tilde{\xi}_{k}}{L^{2}+\frac{\mu^{2} f_{0}^{2}}{N^{2}}\left(m^{2}+\frac{1}{4 H^{2}}\right)}
$$

and

$$
e^{v_{i} \tau}=\frac{L^{2}+\frac{\mu^{2} f_{0}^{2}}{N^{2}}\left(m^{2}+\frac{1}{4 H^{2}}\right)}{\left[L^{2}+\frac{\mu^{2} f_{0}^{2}}{N^{2}}\left(m^{2}+\frac{1}{4 H^{2}}\right)\right] \cos \left(v_{r} \tau\right)-\tau \mu^{2} \beta \tilde{\xi} k \sin \left(v_{r} \tau\right)}
$$


(a)

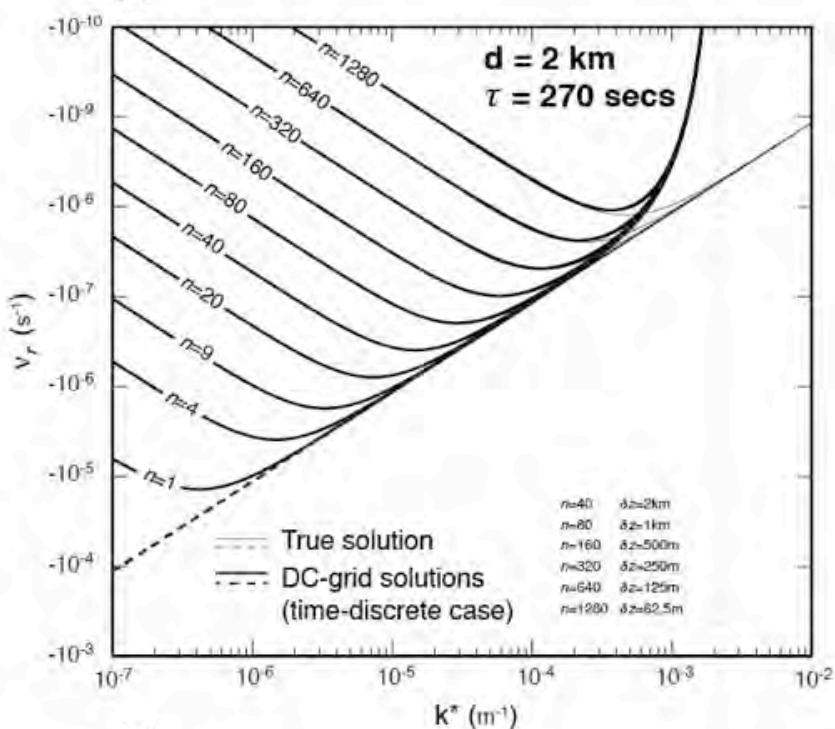

(c)

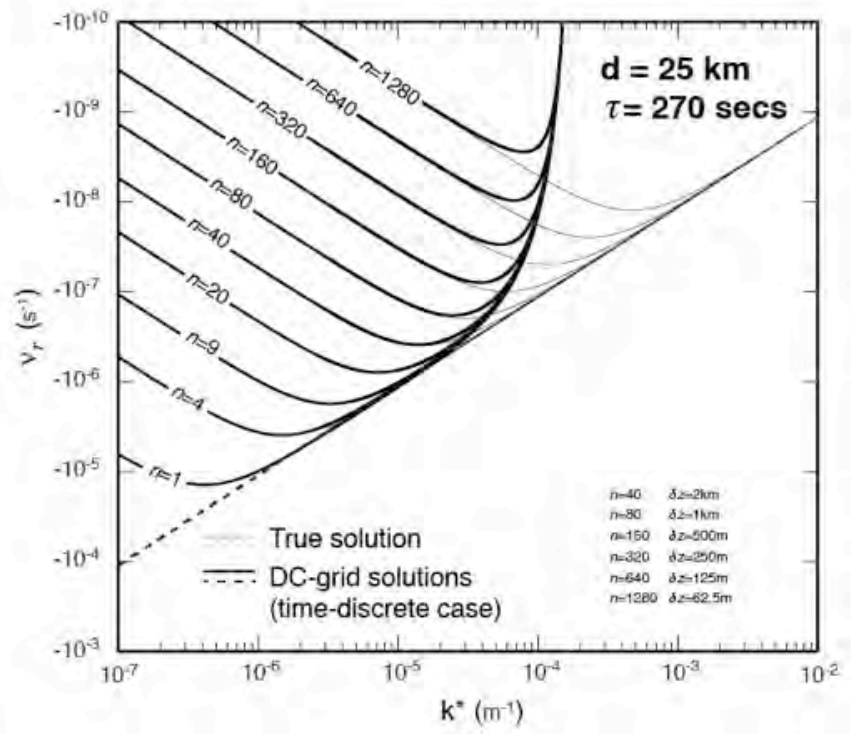

(b)

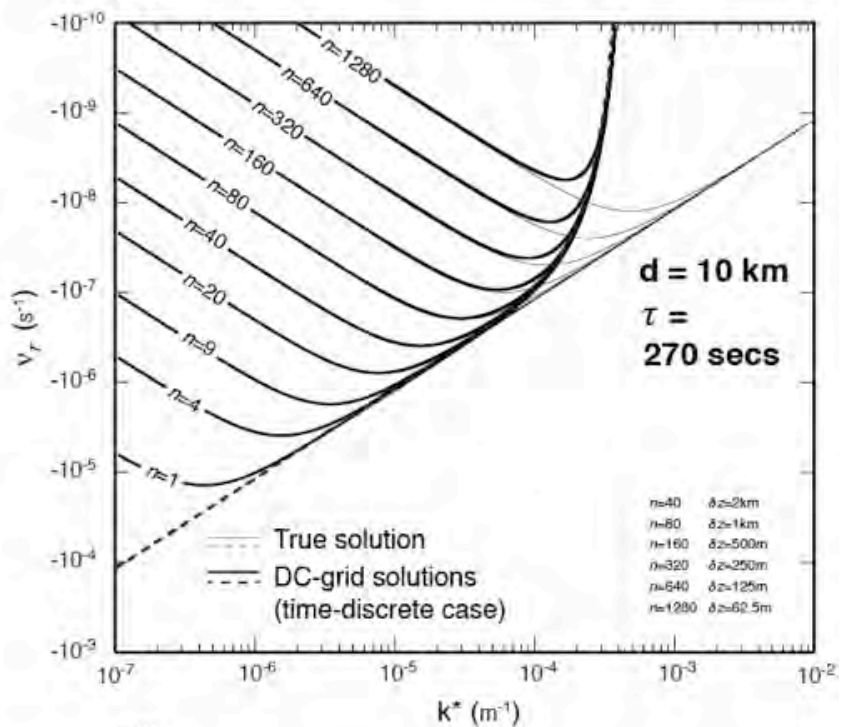

(d)

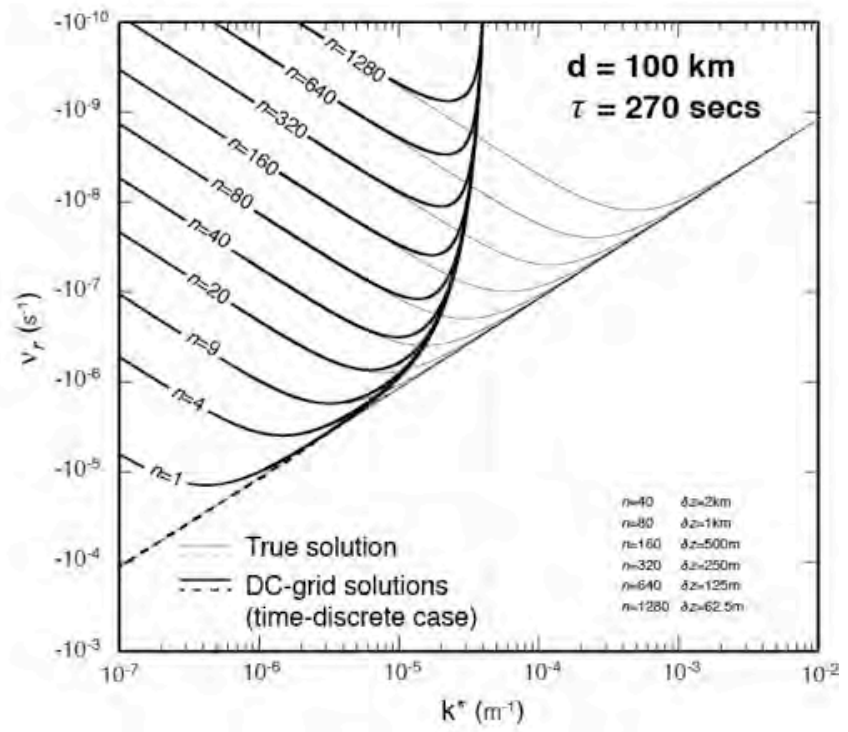

Dispersion of Rossby modes on the DC-grid. 
Anelastic, Quasi-hydrostatic and Lamb wave (or shallow-water) solutions: Nonhydrostatic effects

We write the linearized fully compressible equations as

$$
\begin{gathered}
\frac{\partial u}{\partial t}=-\frac{\partial}{\partial x}\left(\frac{p}{\rho_{0}}\right)+f v, \frac{\partial v}{\partial t}=-\frac{\partial}{\partial y}\left(\frac{p}{\rho_{0}}\right)-f u \text { and } \frac{\partial w}{\partial t}=-\frac{1}{\rho_{0}} \frac{\partial p}{\partial z}-g\left(\frac{\rho}{\rho_{0}}\right) \\
\frac{\partial}{\partial t}\left(g \frac{\theta}{\theta_{0}}\right)=-N^{2} w \text { where } N^{2} \equiv \frac{g \kappa}{H} \\
\frac{\partial \rho}{\partial t}=-\rho_{0} \frac{\partial u}{\partial x}-\rho_{0} \frac{\partial v}{\partial y}-\frac{\partial\left(\rho_{0} w\right)}{\partial z} \\
\frac{1}{c_{S}^{2}} \frac{p}{\rho_{0}}=\frac{\rho}{\rho_{0}}+\frac{\theta}{\theta_{0}} \text { where } c_{S}^{2} \equiv \gamma g H \text { and } \gamma \equiv \frac{1}{1-\kappa}
\end{gathered}
$$

The vorticity-divergence form of these equations are

$$
\begin{gathered}
\frac{\partial \omega_{z}}{\partial t}=-f D \\
\frac{\partial D}{\partial t}=-\left(\frac{\partial^{2}}{\partial x^{2}}+\frac{\partial^{2}}{\partial y^{2}}\right)\left(\frac{p}{\rho_{0}}\right)+f \omega_{z} \\
\frac{\partial w}{\partial t}=-\frac{\partial}{\partial z}\left(\frac{p}{\rho_{0}}\right)+\frac{\kappa}{H}\left(\frac{p}{\rho_{0}}\right)+B \quad \text { where we used } \frac{1}{\rho_{0}} \frac{\partial \rho_{0}}{\partial z}=-\frac{1}{H} \text { and } B \equiv g \frac{\theta}{\theta_{0}}
\end{gathered}
$$




$$
\frac{\partial B}{\partial t}=-N^{2} w \quad\left[\text { or } \frac{\partial}{\partial t}\left(\frac{\theta}{\theta_{0}}\right)=-\frac{\kappa}{H} w\right]
$$

Using (QH.4)and $\frac{1}{\rho_{0}} \frac{\partial \rho_{0}}{\partial z}=-\frac{1}{H}$ in (QH.3), we rewrite the continuity equation as

$$
\frac{1}{\gamma g H} \frac{\partial}{\partial t}\left(\frac{p}{\rho_{0}}\right)=-\left[\frac{\partial u}{\partial x}+\frac{\partial v}{\partial y}+\left(\frac{\partial}{\partial z}+\frac{\kappa}{H}\right) w\right]+\frac{1}{H} w
$$

We rewrite (QH.5)-(QH.9) as

$$
\begin{gathered}
\frac{\partial}{\partial t}\left(\rho_{0}^{1 / 2} \omega_{z}\right)=-f\left(\rho_{0}^{1 / 2} D\right) \\
\frac{\partial}{\partial t}\left(\rho_{0}^{1 / 2} D\right)=-\left(\frac{\partial^{2}}{\partial x^{2}}+\frac{\partial^{2}}{\partial y^{2}}\right)\left(\rho_{0}^{-1 / 2} p\right)+f\left(\rho_{0}^{1 / 2} \omega_{z}\right) \\
\frac{\partial\left(\rho_{0}^{1 / 2} w\right)}{\partial t}=-\left(\frac{\partial}{\partial z}+\frac{1}{2 H}\right)\left(\rho_{0}^{-1 / 2} p\right)+\frac{\kappa}{H}\left(\rho_{0}^{-1 / 2} p\right)+\left(\rho_{0}^{1 / 2} B\right) \\
\frac{\partial}{\partial t}\left(\rho_{0}^{1 / 2} B\right)=-N^{2}\left(\rho_{0}^{1 / 2} w\right) \\
\frac{\partial}{\gamma g H} \frac{\partial t}{\partial t}\left(\rho_{0}^{-1 / 2} p\right)=-\left(\rho_{0}^{1 / 2} D\right)-\left(\frac{\partial}{\partial z}-\frac{1}{2 H}\right)\left(\rho_{0}^{1 / 2} w\right)-\frac{\kappa}{H}\left(\rho_{0}^{1 / 2} w\right)
\end{gathered}
$$


In these equations we used $\frac{\partial \rho_{0}^{1 / 2}}{\partial z}=\frac{1}{2}\left(\frac{1}{\rho_{0}} \frac{\partial \rho_{0}}{\partial z}\right) \rho_{0}^{1 / 2}$.

By changing the variables, we rewrite $(\mathrm{QH} .10)-(\mathrm{QH} .14)$ as

$$
\begin{gathered}
\frac{\partial \omega_{z}}{\partial t}=-f D \\
\frac{\partial D}{\partial t}=-\left(\frac{\partial^{2}}{\partial x^{2}}+\frac{\partial^{2}}{\partial y^{2}}\right) P+f \omega_{z} \\
\frac{\partial w}{\partial t}=-\left(\frac{\partial}{\partial z}+\frac{1}{2 H}\right) P+\frac{\kappa}{H} P+B \\
\frac{\partial B}{\partial t}=-N^{2} w \\
\frac{\partial P}{\gamma g H} \frac{\partial t}{\partial t}=-\left(\frac{\partial}{\partial z}-\frac{1}{2 H}\right) w-\frac{\kappa}{H} w
\end{gathered}
$$

These equations produce the dispersion equation for the fully compressible system as

$$
\begin{aligned}
v^{4}-\left\{\left(N^{2}+f^{2}\right)+\right. & \left.\gamma g H\left[m^{2}+\frac{1}{H^{2}}\left(\frac{1}{2}-\kappa\right)^{2}\right]+\gamma g H\left(k^{2}+\ell^{2}\right)\right\} v^{2} \\
& +N^{2} f^{2}+\gamma g H N^{2}\left(k^{2}+\ell^{2}\right)+f^{2} \gamma g H\left[m^{2}+\frac{1}{H^{2}}\left(\frac{1}{2}-\kappa\right)^{2}\right]=0
\end{aligned}
$$

The quasi-hydrostatic equations are (QH.15), (QH.16), (QH.18), (QH.19) and

$$
0=-\left(\frac{\partial}{\partial z}+\frac{1}{2 H}\right) P+\frac{\kappa}{H} P+B
$$


which is obtained by neglecting the time derivative of vertical velocity in (QH.17). The dispersion equation for the quasi-hydrostatic system is

$$
v_{q h_{-} \text {true }}^{2}=f^{2}+\frac{\gamma g H N^{2}}{N^{2}+\gamma g H\left[m^{2}+\left(\frac{1}{2 H}-\frac{\kappa}{H}\right)^{2}\right]}\left(k^{2}+\ell^{2}\right)
$$

By assuming $B=0$ (and $w=0)$ in (QH.15), (QH.16), (QH.18) and (QH.19), we obtain the dispersion relation for the Lamb wave as

$$
v^{2}=f^{2}+g H^{*}\left(k^{2}+\ell^{2}\right)
$$

where $H^{*} \equiv \gamma H$. Eq. $(\mathrm{QH} .23)$ is identical to the dispersion of the gravity waves with the shallow-water equations for a depth of fluid $H^{*}$. By using $\lambda \equiv \sqrt{g H^{*}} / f,(\mathrm{QH} .23)$ can be written as

$$
v_{s w-t r u e}^{2}=f^{2}+f^{2} \lambda^{2}\left(k^{2}+\ell^{2}\right)
$$

Fig. QH1 shows the true frequencies for the anelastic, quasi-hydrostatic inertia-gravity modes and the Lamb wave. The frequencies for the anelastic and quasi-hydrostatic modes are given by

$$
v_{\text {an_true }}^{2}=\frac{N^{2}\left(k^{2}+\ell^{2}\right)+f^{2}\left[m^{2}+\left(\frac{1}{2 H}\right)^{2}\right]}{\left(k^{2}+\ell^{2}\right)+\left[m^{2}+\left(\frac{1}{2 H}\right)^{2}\right]} \text { (Anelastic) }
$$

and (QH.22), respectively. The frequency for the Lamb wave is given by (QH.23). 


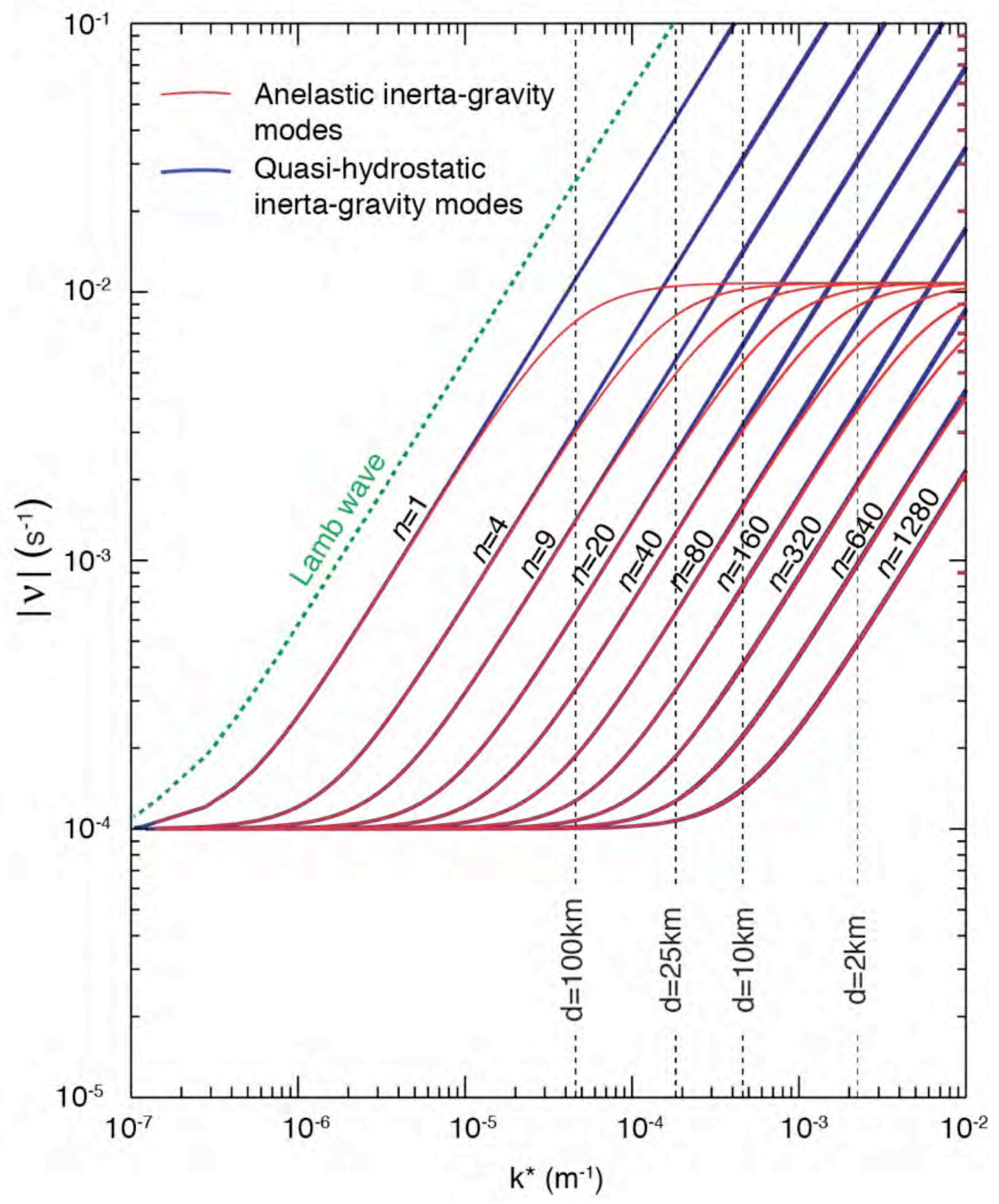

Fig. QH1. Frequencies of the quasi-hydrostatic and anelastic inertia-gravity modes and Lamb wave. Thin black dashed lines indicate the SRHS for the $\mathrm{d}=2 \mathrm{~km}, 10 \mathrm{~km}, 25 \mathrm{~km}$ and $100 \mathrm{~km}$ horizontal grid spacings. 
We define a nonhydrostaticness index as

$$
\mathcal{N}_{h} \equiv \frac{v_{q h_{\text {_true }}}}{v_{\text {an_true }}}-1
$$

Fig. QH2 shows the nonhydrostaticness index for the inertia-gravity modes as a function of the composite horizontal wavenumber and vertical integer wavenumber. The vertically deep modes are generally more nonhydrostatic compared to the shallow modes, and the nonhydrostaticness increases with increasing horizontal wavenumber.

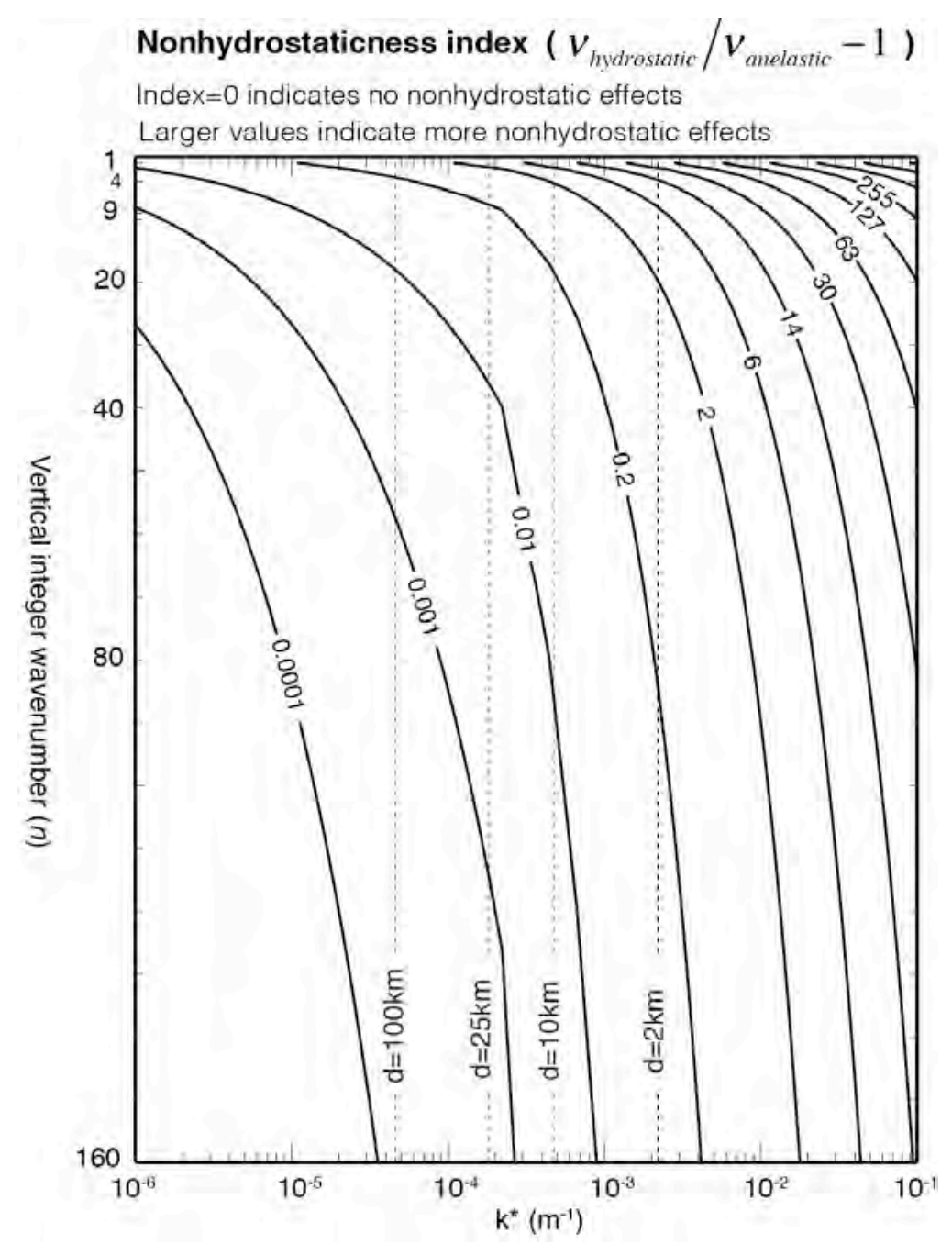

Fig. QH2.

AN, QH, Lamb wave and shallow-water solutions: Nonhydrostatic effects, page 6 
Why nonhydrostaticness shows a pattern shown in Fig. QH2 can also be seen in Fig. $\mathrm{QH} 1$. The frequency of the anelastic inertia-gravity modes is bounded by the Brunt-Vaisala frequency at the upper end. The frequency of the quasi-hydrostatic inertia-gravity modes, however, is not bounded. What is seen in Fig. QH2 is a reflection of the impact of the upper bound brought by the nonhydrostatic effects in the anelastic modes. The nonhydrostatic effects slow down the inertia-gravity waves with short horizontal and long vertical scales, and the group velocity of these modes is nearly zero. This has a substantial effect on the reduction of discretization errors due to finite-difference approximation in the anelastic system compared to the quasi-hydrostatic system, which we will examine next. The discretization errors due to variable averaging are not effected.

For the $\mathrm{C}$ and $\mathrm{Z}$ grids, Eqs. (QH.22), (QH.23) and (QH.25) can be written as

C-grid:

$$
v_{\text {qh_C-grid }}^{2}=\mu^{2} f^{2}+\frac{\gamma g H N^{2}}{N^{2}+\gamma g H\left[m^{2}+\left(\frac{1}{2 H}-\frac{\kappa}{H}\right)^{2}\right]}\left(\xi^{2} k^{2}+\eta^{2} \ell^{2}\right)
$$

and

$$
v_{s w_{-}-\text {- grid }}^{2}=\mu^{2} f^{2}+g H^{*}\left(\xi^{2} k^{2}+\eta^{2} \ell^{2}\right) \quad\left[\text { and } v_{s w_{-}-\text {grid }}^{2}=\mu^{2} f^{2}+f^{2} \lambda^{2}\left(\xi^{2} k^{2}+\eta^{2} \ell^{2}\right)\right]
$$

and

$$
v_{\text {an_C-grid }}^{2}=\frac{N^{2}\left(\xi^{2} k^{2}+\eta^{2} \ell^{2}\right)+\mu^{2} f^{2}\left[m^{2}+\left(\frac{1}{2 H}\right)^{2}\right]}{\left(\xi^{2} k^{2}+\eta^{2} \ell^{2}\right)+\left[m^{2}+\left(\frac{1}{2 H}\right)^{2}\right]} \text { (anelastic) }
$$


where the definitions of $\mu, \xi$ and $\eta$ are given in Section that discusses the C-grid.

Z-grid:

$$
v_{q h_{-} Z \text {-grid }}^{2}=f^{2}+\frac{\gamma g H N^{2}}{N^{2}+\gamma g H\left[m^{2}+\left(\frac{1}{2 H}-\frac{\kappa}{H}\right)^{2}\right]}\left(\xi^{2} k^{2}+\eta^{2} \ell^{2}\right)
$$

and

$$
\begin{gathered}
\left.v_{q h_{-} Z-\text { grid }}^{2}=f^{2}+\gamma g H\left(\xi^{2} k^{2}+\eta^{2} \ell^{2}\right) \text { [and } v_{q h_{-} Z-\text { grid }}^{2}=f^{2}+f^{2} \lambda^{2}\left(\xi^{2} k^{2}+\eta^{2} \ell^{2}\right)\right] \\
v_{\text {an_ Z-grid }}^{2}=\frac{N^{2}\left(\xi^{2} k^{2}+\eta^{2} \ell^{2}\right)+f^{2}\left[m^{2}+\left(\frac{1}{2 H}\right)^{2}\right]}{\left(\xi^{2} k^{2}+\eta^{2} \ell^{2}\right)+\left[m^{2}+\left(\frac{1}{2 H}\right)^{2}\right]} \text { (anelastic) }
\end{gathered}
$$

\section{Anelastic vs. quasi-hydrostatic and shallow-water analyses}

Now we examine the differences between the use of the anelastic, quasi-hydrostatic and shallow-water equations to assess the discretization errors with the $Z$ and $C$ grids. We define an error in the form of the ratio of discrete to true frequencies (hereafter discretization ratio-error) as

$$
r_{a n_{-} Z-\text { grid }} \equiv \frac{v_{\text {an_Z-grid }}}{V_{\text {an_true }}}
$$

Similarly we define $r_{q h_{-} Z-\text { grid }}$ and $r_{s w_{Z} Z-\text { grid }}$, and also the ones corresponding to the C-grid. We also define the difference between these errors that are obtained by the anelastic and quasihydrostatic systems as 


$$
\delta_{a n-q h_{-} Z-g r i d} \equiv r_{a n_{-} Z-\text { grid }}-r_{q h_{-} Z-\text { grid }}
$$

Additionally, we define $\delta_{a n-q h_{-}-\text {-grid }} \equiv r_{a n_{-} C-\text { grid }}-r_{q h_{-} C-\text { grid }}$.

The errors due to finite-difference approximations in discrete dispersion equations (QH. $27)-\left(\mathrm{QH} .31^{\prime}\right)$ are represented by the factors $\xi$ and $\eta$, which modify the horizontal wavenumbers. Since they are always equal to or smaller than the unity, being close to the unity for small horizontal wavenumbers and being the smallest at the SRHS, the modified wavenumbers with discretization are effectively smaller than the continuous case. Then the discrete frequency is equal to the true frequency corresponding to a smaller horizontal wavenumber. For $Z$ and $C$ grids, this is approximately $10 \%$ reduction of wavenumber. Naturally, if the frequency is not changing with the horizontal wavenumber, as with the anelastic case in the high horizontal wavenumber range, the discretization errors remain very small. The magnitude of finite-difference errors should depend on the magnitude of $\partial|v| / \partial k^{*}$, which is also known as the horizontal group velocity.

We present tables of the discretization ratio-errors at the SHRS for the anelastic (Table QH.1), quasi-hydrostatic (Table QH.2) and shallow-water (Table QH.3) systems. The SHRS is selected because the discretization errors are expected to be the largest. Our motivation is to identify the differences, if any, between the use of the anelastic equations and the quasihydrostatic and shallow-water equations in the assessment of discretization errors.

\section{Findings:}

1) The $Z$ grid with the anelastic system produces overall the least errors among the three systems (Tables QH1, QH2 and QH3).

2) The errors of the Z-grid increases with the increasing vertical wavenumbers (of degreasing fluid depth in the shallow-water system) for $d=2 \mathrm{~km}$ and $d=10 \mathrm{~km}$. The errors for the $d=25 \mathrm{~km}$ and $d=100 \mathrm{~km}$ and for very high vertical wavenumbers (say $n=1280$ ) are very small again because the Z-grid cannot generate frequencies less than the rotational frequency (see Eqs. 
QH.29 QH. 31 QH. 31' where the Coriolis term is not averaged). There is no room between the true and Z-grid frequencies at $d=25 \mathrm{~km}$ and $d=100 \mathrm{~km}$ (see Fig. QH1).

3) At the high horizontal wavenumber end, the errors of the Z-grid decreases in the anelastic case, particularly for the deep modes, because the anelastic frequency levels at the BruntVaisala frequency which decreases finite-difference approximation errors.

4) The C-grid produces the least errors with the anelastic system. The C-grid produces both averaging and finite-difference errors. Again these errors are smaller with the nonhydrostatic anelastic system than the quasi-hydrostatic system.

5) If we use the quasi-hydrostatic and the shallow-water equations to assess the discretization errors with the $Z$ and $C$ grids for the deep modes (or deep fluid depths), we can find higher discretization errors (approximately 10\%) than the anelastic system. The numbers $\left(\delta_{a n-q h}\right)$ within the parentheses in Table QH.2 indicates the difference. The difference between the results for the anelastic and quasi-hydrostatic (and shallow-water) systems seems to be related to the nonhydrostaticness (index increases with increasing nonhydrostaticness). 


\section{(Discrete / True) frequencies at SRHS}

(Group velocity $\partial|v| / \partial k^{*}$ )

\begin{tabular}{|c|c|c|c|c|}
\hline \multirow{4}{*}{$\begin{array}{c}\text { Vertical } \\
\text { wave- } \\
\text { number } \\
(n)\end{array}$} & \multicolumn{4}{|c|}{ Anelastic (nonhydrostatic) } \\
\hline & \multicolumn{4}{|c|}{ Horizontal grid spacing } \\
\hline & $\mathrm{d}=100 \mathrm{~km}$ & $d=25 \mathrm{~km}$ & $d=10 \mathrm{~km}$ & $d=2 \mathrm{~km}$ \\
\hline & $r_{\text {an_Z-grid }} \quad r_{\text {an_C-grid }}$ & $r_{a n_{-} Z-\text { grid }} \quad r_{a n \_c-g r i d}$ & $r_{a n \_Z-g r i d} \quad r_{a n \_C-g r i d}$ & $r_{\text {an_Z-grid }} \quad r_{a n \_C-g r i d}$ \\
\hline$\left(\partial|v| / \partial k^{*}\right)$ & 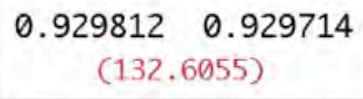 & 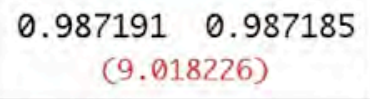 & $\begin{array}{c}0.997710 \quad 0.997709 \\
(0.668803)\end{array}$ & 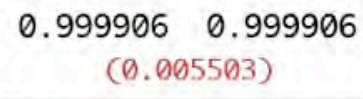 \\
\hline$\left(\partial|v| / \partial k^{*}\right)$ & $\begin{array}{c}0.905303 \quad 0.876828 \\
(13.37373)\end{array}$ & 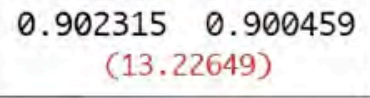 & 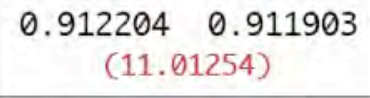 & 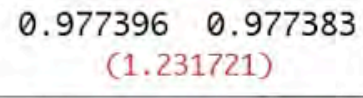 \\
\hline$\left(\partial|v| / \partial k^{*}\right)$ & $\begin{array}{c}0.978224 \quad 0.428102 \\
(0.817474)\end{array}$ & $\begin{array}{c}0.918278 \quad 0.816825 \\
(1.560835)\end{array}$ & $\begin{array}{c}0.903827 \quad 0.885334 \\
(1.685622)\end{array}$ & $\begin{array}{c}0.905467 \quad 0.904704 \\
(1.570003)\end{array}$ \\
\hline $\begin{array}{c}1280 \\
\left(\partial|v| / \partial k^{*}\right)\end{array}$ & $\begin{array}{c}0.999566 \quad 0.060701 \\
(0.014483)\end{array}$ & $\begin{array}{c}0.993492 \quad 0.234918 \\
(0.056128)\end{array}$ & $\begin{array}{c}0.969795 \quad 0.504061 \\
(0.120420)\end{array}$ & $\begin{array}{c}0.908782 \quad 0.863333 \\
(0.205937)\end{array}$ \\
\hline
\end{tabular}

Table QH.1. Tabulation of the frequency errors in a form of the ratio of the discrete to true solutions with the $Z$ and $C$ grids $\left(r_{a n_{-} Z-\text { grid }}\right.$ and $\left.r_{a n_{-} C-\text { grid }}\right)$ for the four SRHS' $(\mathrm{d}=2 \mathrm{~km}, 10 \mathrm{~km}$, $25 \mathrm{~km}$ and $100 \mathrm{~km}$ ), and the four vertical integer wavenumbers. Blue numbers corresponds to the errors of the discrete frequencies that are lower than the rotational frequency $(f)$. Red numbers are the group velocity. 


\section{(Discrete / True) frequency at SRHS}

(Group velocity $\partial|v| / \partial k^{*}$ )

\begin{tabular}{|c|c|c|c|c|}
\hline \multirow{4}{*}{$\begin{array}{c}\text { Vertical } \\
\text { wave- } \\
\text { number } \\
(n)\end{array}$} & \multicolumn{4}{|c|}{ Quasi-hydrostatic } \\
\hline & \multicolumn{4}{|c|}{ Horizontal grid spacing } \\
\hline & $d=100 \mathrm{~km}$ & $d=25 \mathrm{~km}$ & $\mathrm{~d}=10 \mathrm{~km}$ & $d=2 \mathrm{~km}$ \\
\hline & $r_{q h_{-} Z \text {-grid }} \quad r_{q h_{-} C-\text {-grid }}$ & $r_{q h_{-} Z-\text { grid }} \quad r_{q h_{-}-C_{-g r i d}}$ & $r_{q h_{-} z-\text { grid }}$ & $r_{q h_{-} Z \text {-grid }}$ \\
\hline 1 & $0.899834 \quad 0.899739$ & $0.899817 \quad 0.899811$ & $0.900134 \quad 0.900133$ & $0.900261 \quad 0.900261$ \\
\hline$\left(\delta_{a t-q h}\right)$ & $(0.0299) \quad(0.0299)$ & $(0.0873) \quad(0.0873)$ & $(0.0975) \quad(0.0975)$ & $(0.0996) \quad(0.0996)$ \\
\hline$\left(\partial|v| / \partial k^{*}\right)$ & $(243.2004)$ & (243.2199) & $(243.2210)$ & $(243.2212)$ \\
\hline 20 & $0.905165 \quad 0.876695$ & $0.900168 \quad 0.898317$ & $0.900190 \quad 0.899893$ & $0.900263 \quad 0.900251$ \\
\hline$\left(\delta_{a n-q h}\right)$ & $(0.0001) \quad(0.0001)$ & $(0.0021) \quad(0.0021)$ & $(0.0120) \quad(0.0120)$ & $(0.0771) \quad(0.0771)$ \\
\hline$\left(\partial|v| / \partial k^{*}\right)$ & $(13.37363)$ & $(13.22659)$ & $(13.75793)$ & (13.76146) \\
\hline 160 & $0.978221 \quad 0.428101$ & $0.918243 \quad 0.816794$ & $0.903613 \quad 0.885124$ & $0.900405 \quad 0.899646$ \\
\hline$\left(\delta_{a n-q h}\right)$ & $(0.0000) \quad(0.0000)$ & $(0.0000) \quad(0.0000)$ & $(0.0002) \quad(0.0002)$ & $(0.0050) \quad(0.0050)$ \\
\hline$\left(\partial|v| / \partial k^{*}\right)$ & $(0.817474)$ & $(1.560835)$ & $(1.692092)$ & $(1.719620)$ \\
\hline 1280 & $0.999566 \quad 0.060701$ & 0.993492 & 0.969791 & $0.908698 \quad 0.863251$ \\
\hline$\left(\delta_{a n-q h}\right)$ & $(0.0000) \quad(0.0000)$ & $(0.0000) \quad(0.0000)$ & $(0.0000) \quad(0.0000)$ & $(0.0000) \quad(0.0000)$ \\
\hline$\left(\partial v \mid / \partial k^{*}\right)$ & $(0.014483)$ & $(0.056128)$ & $(0.120438)$ & $(0.206257)$ \\
\hline
\end{tabular}

Table QH.2. Tabulation of the frequency errors in a form of the ratio of the discrete to true solutions with the $Z$ and C grids $\left(r_{q h_{-} Z-\text { grid }}\right.$ and $\left.r_{q h_{-}-\text {grid }}\right)$ for the four SRHS' (d=2km, $10 \mathrm{~km}$, $25 \mathrm{~km}$ and $100 \mathrm{~km}$ ), and the four vertical integer wavenumbers. Blue numbers corresponds to the errors of the discrete frequencies that are lower than the rotational frequency $(f)$. Numbers within the parentheses are the difference of errors between the anelastic (tabulated in Table QH.1) and quasi-hydrostatic systems. 


\section{Discrete / True frequency at SRHS}

\begin{tabular}{|c|c|c|c|c|}
\hline \multirow{4}{*}{$\begin{array}{c}\text { Vertical } \\
\text { wave- } \\
\text { number } \\
(n)\end{array}$} & \multicolumn{4}{|c|}{ Shallow-water } \\
\hline & \multicolumn{4}{|c|}{ Horizontal grid spacing } \\
\hline & $d=100 \mathrm{~km}$ & $\mathrm{~d}=25 \mathrm{~km}$ & $\mathrm{~d}=10 \mathrm{~km}$ & $\mathrm{~d}=2 \mathrm{~km}$ \\
\hline & 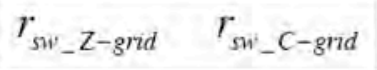 & $r_{s w_{-} z-\text { grid }} \quad r_{s w_{-}-C_{-g} \text { grid }}$ & $r_{\text {sw_Z-grid }} \quad r_{s w_{-} C-\text { grid }}$ & 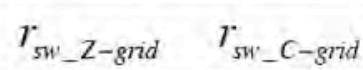 \\
\hline $\begin{array}{l}H=24 \mathrm{~km} \\
(\lambda / d)\end{array}$ & $\begin{array}{c}0.8998190 .899802 \\
(57)\end{array}$ & $\begin{array}{c}0.899816 \quad 0.899815 \\
(229)\end{array}$ & $\begin{array}{c}0.900134 \quad 0.900134 \\
(574)\end{array}$ & $\begin{array}{c}0.9002610 .900261 \\
(2870)\end{array}$ \\
\hline $\begin{array}{l}H=1 \mathrm{~m} \\
(\lambda / d)\end{array}$ & $\begin{array}{c}0.943611 \quad 0.682811 \\
(0.37)\end{array}$ & $\begin{array}{c}0.904459 \quad 0.879791 \\
(1.48)\end{array}$ & $\begin{array}{c}0.900905 \quad 0.896834 \\
(3.70)\end{array}$ & $\begin{array}{c}0.900292 \quad 0.900128 \\
(18.52)\end{array}$ \\
\hline $\begin{array}{c}H=0.01 \mathrm{~m} \\
(\lambda / d)\end{array}$ & $\begin{array}{c}0.998724 \quad 0.104136 \\
(0.037)\end{array}$ & $\begin{array}{c}0.9828710 .380109 \\
(0.148)\end{array}$ & $\begin{array}{c}0.943803 \quad 0.682950 \\
(0.370)\end{array}$ & $\begin{array}{c}0.903272 \quad 0.887271 \\
(1.852)\end{array}$ \\
\hline
\end{tabular}

Table QH.3. Tabulation of the frequency errors in a form of the ratio of the discrete to true solutions with the $Z$ and $C$ grids $\left(r_{s w_{-} Z-\text { grid }}\right.$ and $\left.r_{s w_{-} C-\text { grid }}\right)$ for the four SRHS' $(\mathrm{d}=2 \mathrm{~km}, 10 \mathrm{~km}$, $25 \mathrm{~km}$ and $100 \mathrm{~km}$ ), and for three fluid depths. Blue numbers corresponds to the errors of the discrete frequencies that are lower than the rotational frequency $(f)$. Numbers within the parentheses are the corresponding Rossby radius of deformation divided by the grid spacing. 
Baroclinic Rossby Modes with the Fully Compressible, Quasi-hydrostatic and Anelastic Systems

1- Basic linearized equations for a midlatitude $\beta$-plane

$$
\begin{gathered}
\frac{\partial}{\partial t} \frac{\partial u}{\partial x}=-\frac{\partial^{2}}{\partial x^{2}}\left(\frac{p}{\rho_{0}}\right)+\left[f_{0}+\beta\left(y-y_{0}\right)\right] \frac{\partial v}{\partial x} \\
\frac{\partial}{\partial t} \frac{\partial u}{\partial y}=-\frac{\partial^{2}}{\partial x \partial y}\left(\frac{p}{\rho_{0}}\right)+\left[f_{0}+\beta\left(y-y_{0}\right)\right] \frac{\partial v}{\partial y}+\beta v \\
\frac{\partial}{\partial t} \frac{\partial v}{\partial x}=-\frac{\partial^{2}}{\partial y \partial x}\left(\frac{p}{\rho_{0}}\right)-\left[f_{0}+\beta\left(y-y_{0}\right)\right] \frac{\partial u}{\partial x} \\
\frac{\partial}{\partial t} \frac{\partial v}{\partial y}=-\frac{\partial^{2}}{\partial y^{2}}\left(\frac{p}{\rho_{0}}\right)-\left[f_{0}+\beta\left(y-y_{0}\right)\right] \frac{\partial u}{\partial y}-\beta u
\end{gathered}
$$

With the assumption $f_{0} \gg \beta\left(y-y_{0}\right)$, We write vorticity and divergence equations as

$$
\begin{gathered}
\frac{\partial}{\partial t}\left(-\frac{\partial u}{\partial y}+\frac{\partial v}{\partial x}\right)=-f_{0}\left(\frac{\partial u}{\partial x}+\frac{\partial v}{\partial y}\right)-\beta v \\
\frac{\partial}{\partial t}\left(\frac{\partial u}{\partial x}+\frac{\partial v}{\partial y}\right)=-\left(\frac{\partial^{2}}{\partial x^{2}}+\frac{\partial^{2}}{\partial y^{2}}\right)\left(\frac{p}{\rho_{0}}\right)+f_{0}\left(-\frac{\partial u}{\partial y}+\frac{\partial v}{\partial x}\right)-\beta u
\end{gathered}
$$

The vertical momentum, thermodynamic and continuity equations are given by

$$
\begin{gathered}
\frac{\partial w}{\partial t}=-\frac{\partial}{\partial z}\left(\frac{p}{\rho_{0}}\right)+\frac{\kappa}{H}\left(\frac{p}{\rho_{0}}\right)+\left(g \frac{\theta}{\theta_{0}}\right) \\
\frac{\partial}{\partial t}\left(g \frac{\theta}{\theta_{0}}\right)=-N^{2} w \\
\frac{1}{\gamma g H} \frac{\partial}{\partial t}\left(\frac{p}{\rho_{0}}\right)=-\left[\frac{\partial u}{\partial x}+\frac{\partial v}{\partial y}+\left(\frac{\partial}{\partial z}+\frac{\kappa}{H}\right) w\right]+\frac{1}{H} w
\end{gathered}
$$


We make following definitions and variable transformations:

$P \equiv \rho_{0}^{-1 / 2} p, B \equiv \rho_{0}^{1 / 2} g\left(\frac{\theta}{\theta_{0}}\right), \rho_{0}^{1 / 2} u \Rightarrow u, \rho_{0}^{1 / 2} v \Rightarrow v$, and $\rho_{0}^{1 / 2} w \Rightarrow w$.

Then, Eqs. (BB.5)-(BB.9) can be written as

$$
\begin{gathered}
\frac{\partial}{\partial t}\left(-\frac{\partial u}{\partial y}+\frac{\partial v}{\partial x}\right)=-f_{0}\left(\frac{\partial u}{\partial x}+\frac{\partial v}{\partial y}\right)-\beta v \\
\frac{\partial}{\partial t}\left(\frac{\partial u}{\partial x}+\frac{\partial v}{\partial y}\right)=-\left(\frac{\partial^{2}}{\partial x^{2}}+\frac{\partial^{2}}{\partial y^{2}}\right) P+f_{0}\left(-\frac{\partial u}{\partial y}+\frac{\partial v}{\partial x}\right)-\beta u \\
\frac{\partial w}{\partial t}=-\left[\frac{\partial}{\partial z}+\frac{1}{H}\left(\frac{1}{2}-\kappa\right)\right] P+B \\
\frac{\partial B}{\partial t}=-N^{2} w \\
\frac{1}{\gamma g H} \frac{\partial P}{\partial t}=-\left(\frac{\partial u}{\partial x}+\frac{\partial v}{\partial y}\right)-\left[\frac{\partial}{\partial z}-\frac{1}{H}\left(\frac{1}{2}-\kappa\right)\right] w
\end{gathered}
$$

Using $\Phi \equiv \hat{\Phi} e^{i(k x+y y+m z-v t)}$

$$
\begin{gathered}
-\ell v \hat{u}+k v \hat{v}=-i f_{0}(k \hat{u}+\ell \hat{v})-\beta \hat{v} \\
k v \hat{u}+\ell v \hat{v}=\left(k^{2}+\ell^{2}\right) \hat{P}+i f_{0}(-\ell \hat{u}+k \hat{v})-\beta \hat{u} \\
-\underset{\sim}{i v \hat{w}}=-\left[\underset{\sim}{i m} m+\frac{1}{H}\left(\frac{1}{2}-\kappa\right)\right] \hat{P}+\hat{B} \\
-\underset{\sim}{i v \hat{B}}=-N^{2} \hat{w} \\
-\underset{\sim}{v} \frac{1}{\gamma g H} \hat{P}=-(\underset{\sim}{i k \hat{u}}+\underset{\sim}{i \ell} \ell \hat{v})-\left[\underset{\sim}{i m}-\frac{1}{H}\left(\frac{1}{2}-\kappa\right)\right] \hat{w}
\end{gathered}
$$


To find a real frequency, we ignore $\beta \hat{u}$ and assume $\ell=0$.

$$
\begin{gathered}
i f_{0} k \hat{u}+(k v+\beta) \hat{v}=0 \\
k v \hat{u}-\underset{\sim}{i} f_{0} k \hat{v}=k^{2} \hat{P} \\
\left(v^{2}-N^{2}\right) \hat{w}=-\underset{\sim}{i v}\left[\underset{\sim}{i m}+\frac{1}{H}\left(\frac{1}{2}-\kappa\right)\right] \hat{P} \\
-\underset{\sim}{i v} \frac{1}{\gamma g H} \hat{P}=-\underset{\sim}{i k \hat{u}}-\left[\underset{\sim}{i} m-\frac{1}{H}\left(\frac{1}{2}-\kappa\right)\right] \hat{w}
\end{gathered}
$$

The dispersion relation for the fully compressible system:

Form (BB.20)-(BB.23), we obtain

$$
\begin{array}{r}
k v^{5}+\beta v^{4}-k\left[N^{2}+f_{0}^{2}+\gamma g H k^{2}+\gamma g H\left(m^{2}+\sigma_{m}^{2}\right)\right] v^{3}-\beta\left[\gamma g H k^{2}+N^{2}+\gamma g H\left(m^{2}+\sigma_{m}^{2}\right)\right] v^{2} \\
+k\left[\gamma g H k^{2} N^{2}+N^{2} f_{0}^{2}+\gamma g H\left(m^{2}+\sigma_{m}^{2}\right) f_{0}^{2}\right] v+\beta \gamma g H k^{2} N^{2}=0
\end{array}
$$

where

$$
\sigma_{m}^{2} \equiv \frac{1}{H^{2}}\left(\frac{1}{2}-\kappa\right)^{2}
$$

The dispersion relation for the quasi-hydrostatic (primitive) system:

For the quasi-hydrostatic (primitive) system, (BB.22) is written as

$$
-N^{2} \hat{w}=-\underset{\sim}{i} v\left[\underset{\sim}{i} m+\frac{1}{H}\left(\frac{1}{2}-\kappa\right)\right] \hat{P}
$$

Using (BB.20), (BB.21), (BB.23) and (BB.26), we obtain the dispersion relation for the quasihydrostatic (primitive) system as

$$
\begin{aligned}
-k\left[N^{2}+\gamma g H\left(m^{2}+\sigma_{m}^{2}\right)\right] v^{3} & -\beta\left[N^{2}+\gamma g H\left(m^{2}+\sigma_{m}^{2}\right)\right] v^{2} \\
+ & k\left[\gamma g H k^{2} N^{2}+N^{2} f_{0}^{2}+\gamma g H\left(m^{2}+\sigma_{m}^{2}\right) f_{0}^{2}\right] v+\beta \gamma g H k^{2} N^{2}=0
\end{aligned}
$$




\section{The dispersion relation for the anelastic system:}

We use Lipps-Hemler anelastic system, in which (BB.22) and (BB.23) are approximated as

$$
\left(v^{2}-N^{2}\right) \hat{w}=-\underset{\sim}{i} v\left[\underset{\sim}{i m}+\frac{1}{H}\left(\frac{1}{2}\right)\right] \hat{P}
$$

and

$$
0=-\underset{\sim}{i k \hat{u}}-\left[\underset{\sim}{\operatorname{im}}-\frac{1}{H}\left(\frac{1}{2}\right)\right] \hat{w},
$$

respectively. From (BB.20), (BB.21), (BB.28) and (BB.29), we obtain the dispersion relation for the anelastic system as

$$
\begin{aligned}
-k\left[\gamma g H k^{2}+\gamma g H\left(m^{2}+\tilde{\sigma}_{m}^{2}\right)\right] v^{3} & -\beta\left[\gamma g H k^{2}+\gamma g H\left(m^{2}+\tilde{\sigma}_{m}^{2}\right)\right] v^{2} \\
+ & k\left[\gamma g H k^{2} N^{2}+\gamma g H\left(m^{2}+\tilde{\sigma}_{m}^{2}\right) f_{0}^{2}\right] v+\beta \gamma g H k^{2} N^{2}=0
\end{aligned},
$$

where

$$
\tilde{\sigma}_{m}^{2} \equiv \frac{1}{4 H^{2}} .
$$

The dispersion relation for the quasi-geostrophic (quasi-static) system:

For comparison, we write the dispersion for the quasi-geostrophic (quasi-static) we used in this study for only zonal modes as

$$
v=\frac{-\beta k}{k^{2}+\frac{f_{0}^{2}}{N^{2}} \tilde{\sigma}_{m}^{2}} .
$$

We found the roots (frequencies) of (BB.24), (BB.27), (BB.30) that are between $-10^{-10}$ and $-10^{-3}\left(-10^{-3} \leq v \leq-10^{-10}\right)$ as a function of the horizontal wavenumber $(k)$ for given vertical wavenumber $(n)$. These roots corresponds to the baroclinic Rossby modes. Also, we computed the frequencies for the baroclinic Rossby modes from (BB.32) for the quasigeostrophic system. Fig. BB.1 shows the frequencies of the baroclinic Rossby modes with the 
fully compressible, quasi-hydrostatic (primitive), anelastic and quasi-geostrophic (quasi-static) system of equations.

(a)

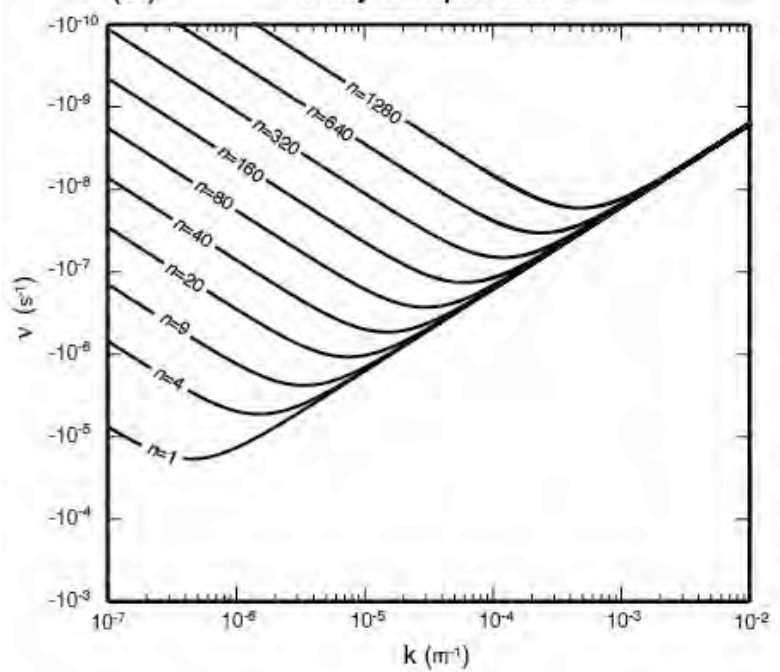

(c)

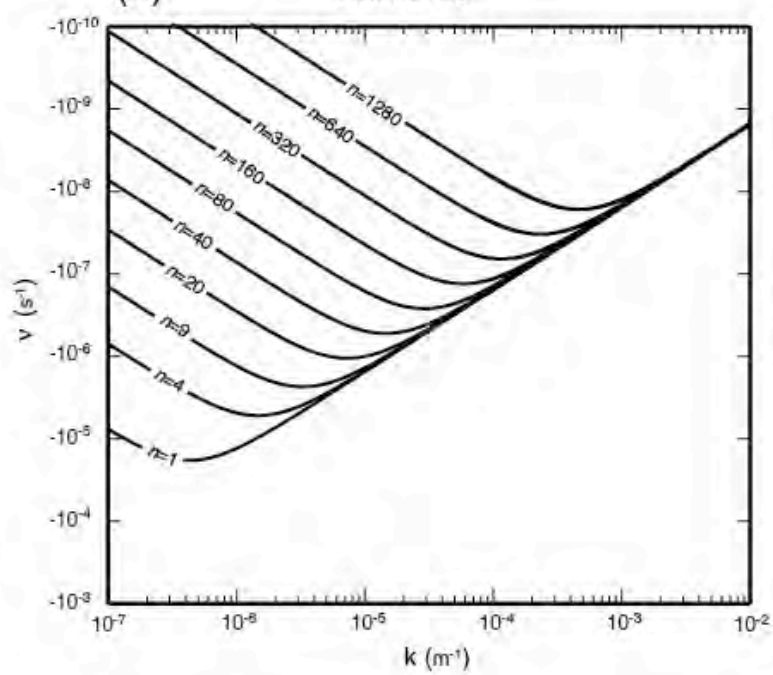

(b) Quasi-hydrostatic (Primitive)

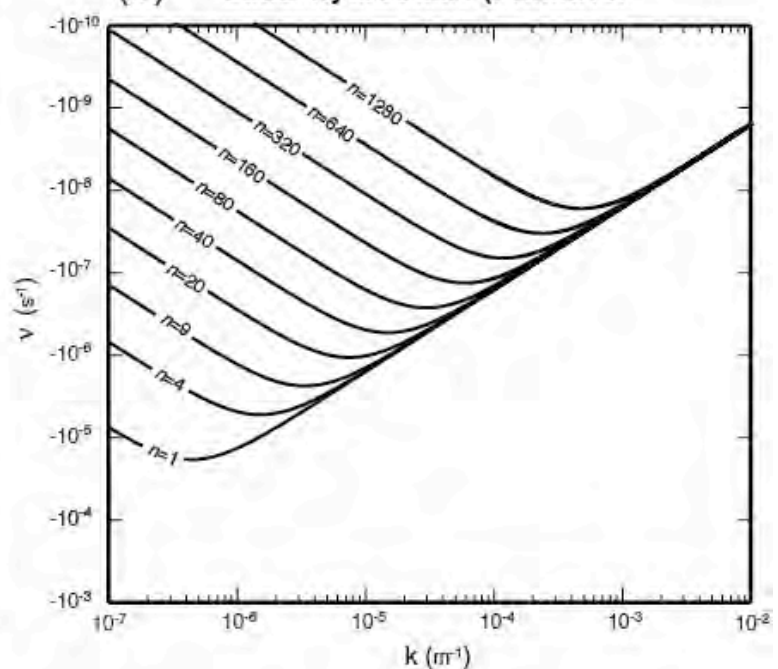

(d) Quasi-geostrophic (Quasi-static)

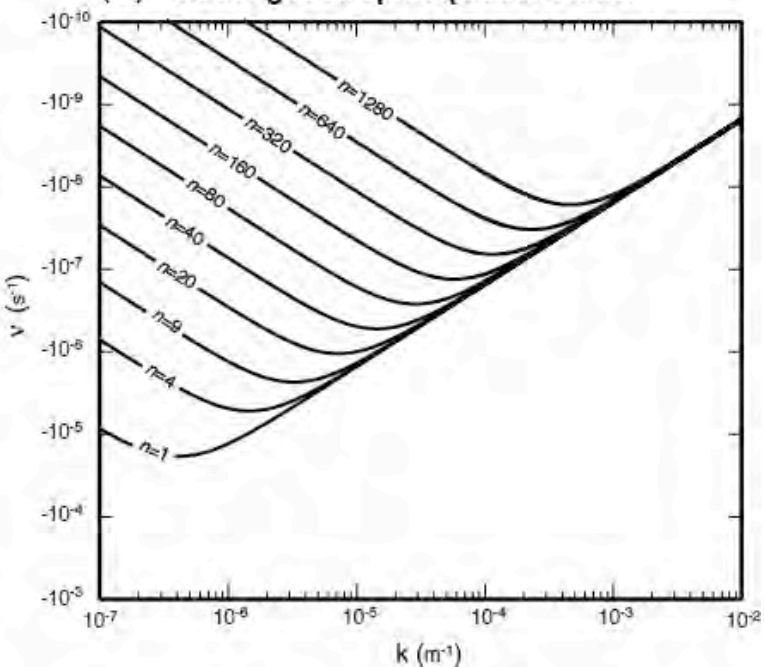

Fig. BB.1. The frequencies of baroclinic Rossby modes with (a) the fully compressible, (b) quasi-hydrostatic (primitive), (c) anelastic, (d) quasi-geostrophic (quasi-static) system of equations.

The frequencies obtained with these systems are virtually identical to each other. This confirms that the baroclinic Rossby modes are not significantly influenced from the nonhydrostatic effects, and therefore, the use of the quasi-geostrophic theory is highly justifiable. 


\section{Numerical Simulations with Noisy Forcing}

Physical processes can be source of noise in atmospheric models. To assess the performance of the Z, C, D and CD grids under such conditions, we made numerical integrations with the models discussed in the numerical integration section, which are modified by adding forcing terms $(\partial \tilde{B} / \partial t)_{\text {forcing }}$ to their buoyancy prediction equations. The equations of the Z-, C-, D- and CD-grid models with the forcing terms are given by

Equations on Z grid:
$\frac{\partial\left(\omega_{z}\right)_{i, j}}{\partial t}=-f D_{i, j}$
$\frac{\partial D_{i, j}}{\partial t}=f\left(\omega_{z}\right)_{i, j}-\left(\tilde{\nabla}_{\mathrm{H}}^{2} P\right)_{i, j}$
$\frac{\partial \tilde{B}_{i, j}}{\partial t}=N^{2} D_{i, j}+\left[(\partial \tilde{B} / \partial t)_{f o r c i n g}\right]_{i, j}$
$\left(\tilde{\nabla}_{\mathrm{H}}^{2} P\right)_{i, j}-\left(m^{2}+\frac{1}{4 H^{2}}\right)_{i, j}=f\left(\omega_{z}\right)_{i, j}+\tilde{B}_{i, j}$
where
$\left(\tilde{\nabla}_{\mathrm{H}}^{2} P\right)_{i, j} \equiv \frac{1}{d^{2}}\left(P_{i+1, j}+P_{i-1, j}+P_{i, j+1}+P_{i, j-1}-4 P_{i, j}\right)$
and
$\tilde{B}_{i, j} \equiv\left(\frac{\partial}{\partial z}-\frac{1}{2 H}\right) B_{i, j}$,




\begin{tabular}{|c|c|}
\hline $\begin{array}{l}\frac{\partial\left(\omega_{z}\right)_{i+\frac{1}{2}, j+\frac{1}{2}}}{\partial t}=-f \bar{D}_{i+\frac{1}{2}, j+\frac{1}{2}} \\
\frac{\partial D_{i, j}}{\partial t}=f\left(\overline{\left.\omega_{z}\right)_{i, j}}-\left(\tilde{\nabla}_{\mathrm{H}}^{2} P\right)_{i, j}\right. \\
\frac{\partial \tilde{B}_{i, j}}{\partial t}=N^{2} D_{i, j}+\left[(\partial \tilde{B} / \partial t)_{f o r c i n g}\right]_{i, j} \\
\left(\tilde{\nabla}_{\mathrm{H}}^{2} P\right)_{i, j}-\left(m^{2}+\frac{1}{4 H^{2}}\right) P_{i, j}=f \overline{\left(\omega_{z}\right)_{i, j}}+\tilde{B}_{i, j} \quad \text { (RF.8) } \\
\text { where }) \\
\left(\tilde{\nabla}_{\mathrm{H}}^{2} P\right)_{i, j} \equiv \frac{1}{d^{2}}\left(P_{i+1, j}+P_{i-1, j}+P_{i, j+1}+P_{i, j-1}-4 P_{i, j}\right), \\
\tilde{B}_{i, j} \equiv\left(\frac{\partial}{\partial z}-\frac{1}{2 H}\right) B_{i, j}, \\
\bar{D}_{i+\frac{1}{2}, j+\frac{1}{2}} \equiv \frac{1}{4}\left(D_{i, j}+D_{i+1, j}+D_{i, j+1}+D_{i+1, j+1}\right)\end{array}$ & 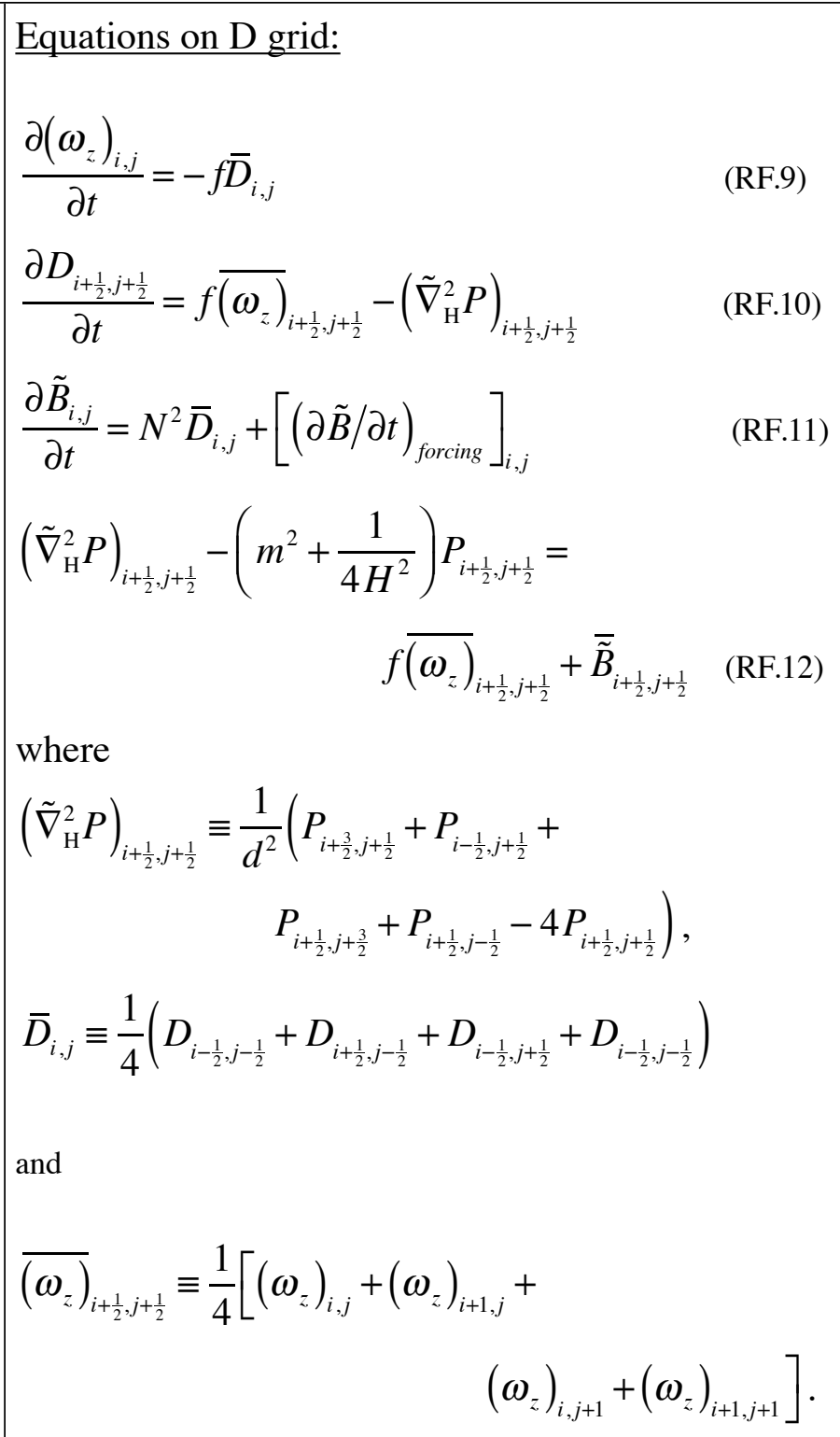 \\
\hline
\end{tabular}




\section{Predictor step (*) on the C-grid:}

$\left(\omega_{z}\right)_{i+1 / 2, j+1 / 2}^{(*)}=\overline{\left(\omega_{z}\right)_{i+1 / 2, j+1 / 2}^{(\mathrm{n})}}-\frac{1}{2} \tau f D_{i+1 / 2, j+1 / 2}^{(\mathrm{n})}$

$D_{i, j}^{(*)}=\bar{D}_{i, j}^{(\mathrm{n})}+\frac{1}{2} \tau\left[\underline{f\left({\overline{()_{z}}}_{i, j}^{(*)}\right.}-\left(\tilde{\nabla}_{\mathrm{H}}^{2} P\right)_{i, j}\right]$

$\tilde{B}_{i, j}^{(*)}=\tilde{B}_{i, j}^{(\mathrm{n})}+\frac{1}{2} \tau N^{2} \bar{D}_{i, j}^{(\mathrm{n})}$

$\left(\tilde{\nabla}_{\mathrm{H}}^{2} P\right)_{i, j}-\left(m^{2}+\frac{1}{4 H^{2}}\right) P_{i, j}=\underline{f \overline{\left(\omega_{z}\right)_{i, j}^{(*)}}}+\tilde{B}_{i, j}^{(*)}$

(RF.16)

where $\left(\tilde{\nabla}_{\mathrm{H}}^{2} P\right)_{i, j}$ is given by (RF.16).

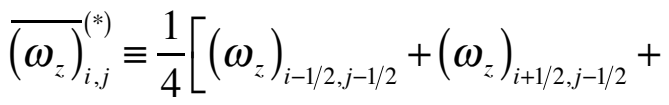

$\bar{D}_{i, j}^{(\mathrm{n})} \equiv \frac{1}{4}\left(D_{i-1 / 2, j-1 / 2}+D_{i+1 / 2, j-1 / 2}+\right.$

$$
\left.D_{i-1 / 2, j+1 / 2}+D_{i-1 / 2, j-1 / 2}\right)^{(\mathrm{n})}
$$

Corrector step $(\mathrm{n}+1)$ on the D-grid:

$\left(\omega_{z}\right)_{i, j}^{(\mathrm{n}+1)}=\left(\omega_{z}\right)_{i, j}^{(\mathrm{n})}-\tau f D_{i, j}^{(*)}$

$D_{i+1 / 2, j+1 / 2}^{(\mathrm{n}+1)}=D_{i+1 / 2, j+1 / 2}^{(\mathrm{n})}+\tau\left[\underline{\underline{f \overline{\left(\omega_{z}\right)^{(\mathrm{n}+1)}}}}-\left(\tilde{\nabla}_{\mathrm{H}}^{2} P\right)\right]_{i+1 / 2, j+1 / 2}$

(RF.18)

$\tilde{B}_{i, j}^{(\mathrm{n}+1)}=\tilde{B}_{i, j}^{(\mathrm{n})}+\tau N^{2} \underline{\underline{\underline{\bar{D}_{i, j}^{(\mathrm{n}+1)}}}}+\tau\left[(\partial \tilde{B} / \partial t)_{\text {forcing }}\right]_{i, j}$

$\left(\tilde{\nabla}_{\mathrm{H}}^{2} P\right)_{i+1 / 2, j+1 / 2}-\left(m^{2}+\frac{1}{4 H^{2}}\right) P_{i+1 / 2, j+1 / 2}=$

$$
\underline{\underline{\left(\omega_{z}\right)_{i+1 / 2, j+1 / 2}^{(n+1)}}}+\overline{\tilde{B}}_{i+1 / 2, j+1 / 2}^{(n+1)}
$$

where $\left(\tilde{\nabla}_{\mathrm{H}}^{2} P\right)_{i+1 / 2, j+1 / 2}$ is given by (RF.20), and

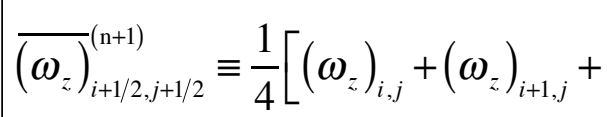

$$
\left.\left(\omega_{z}\right)_{i, j+1}+\left(\omega_{z}\right)_{i+1, j+1}\right]^{(\mathrm{n}+1)}
$$

$\overline{\tilde{B}}_{i+1 / 2, j+1 / 2}^{(\mathrm{n}+1)} \equiv \frac{1}{4}\left(\tilde{B}_{i, j}+\tilde{B}_{i+1, j}+\tilde{B}_{i, j+1}+\tilde{B}_{i+1, j+1}\right)^{(\mathrm{n}+1)}$

A randomly produced buoyancy forcing pattern shown in Fig. BF1, which remains unchanged during integrations, is used to introduce noise to the buoyancy. We prescribe $(\partial \tilde{B} / \partial t)_{\text {forcing }}=\mp 1 s^{-3}$ in these simulations. Note that the domain averaged $(\partial \tilde{B} / \partial t)_{\text {forcing }}$ is virtually zero. The horizontal grid spacing and the vertical wavenumber are $50 \mathrm{~m}$ and $n=320$, respectively. The simulations start from the unperturbed initial prognostic variables. Fig. RF2 shows the buoyancy for the 24, 96 and 168 secs of the integration from the Z-grid and D-grid models. In the D-grid simulation, the noise in the buoyancy expands and the amplitude of the noise steadily increases with time. The time evolution of the maximum and minimum of buoyancy perturbation over the entire horizontal domain from the Z-, C-, D- and CD- 
grid models is shown in Fig. BF3. In the Z- and C-grid simulations, the amplitude of buoyancy perturbation does not increase, which implies that the divergence (or vertical velocity) responds efficiently to counter the forcing. The D- and CD-grid simulations, the divergence response is not efficient enough to prevent the increase of the amplitude of buoyancy perturbation.

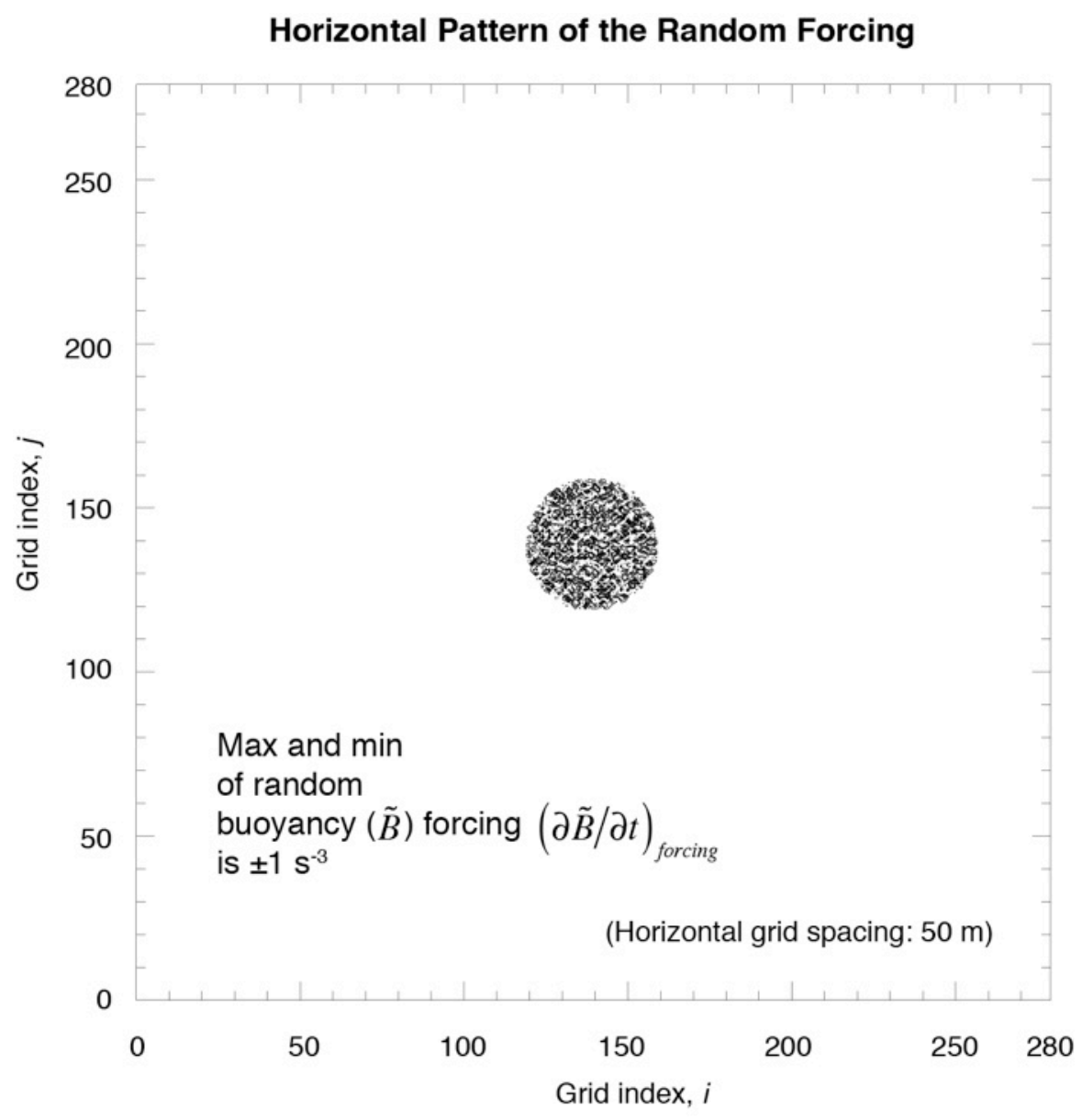

Fig. RF1. 


\section{Buoyancy $(\tilde{B})$ from random forcing}

Z-grid

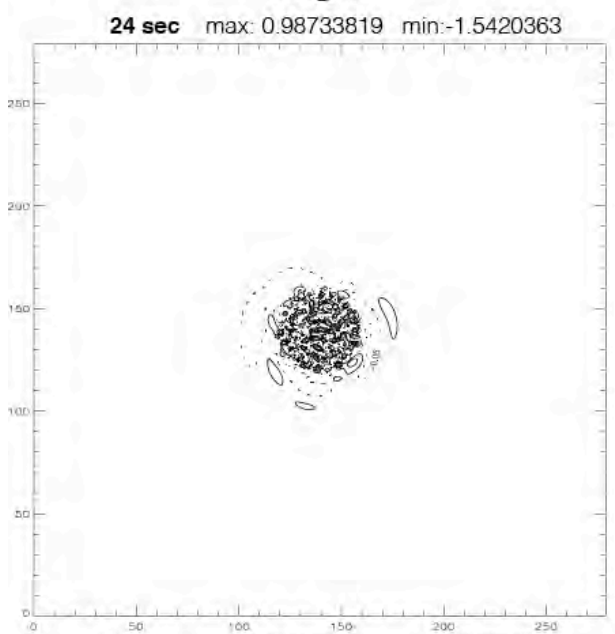

$96 \mathrm{sec} \max : 1.9417721$ min:-1.9998530

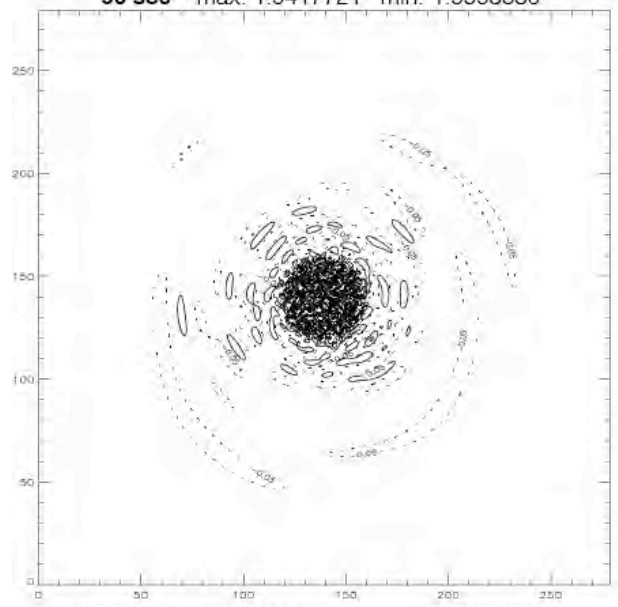

$168 \mathrm{sec} \max : 1.8650047 \min :-2.0247405$

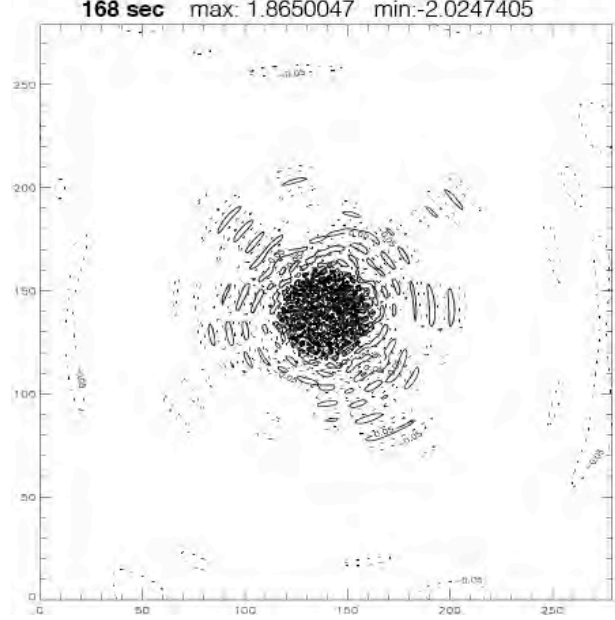

D-grid

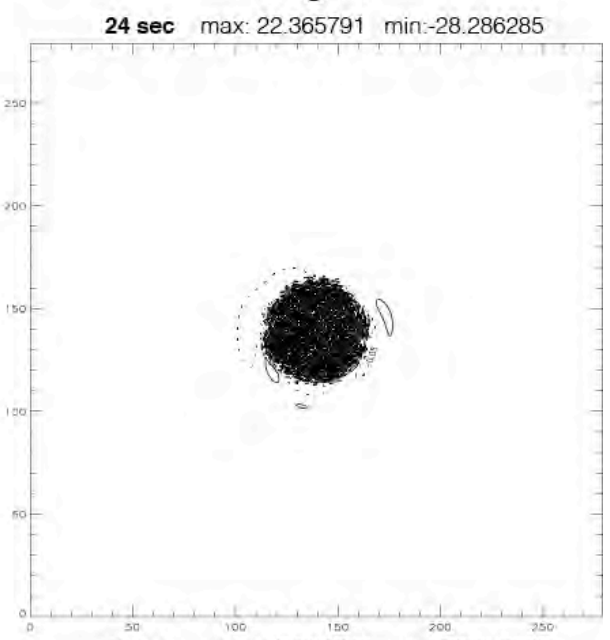

$96 \mathrm{sec}$ max: 59.654346 min:-62.150200

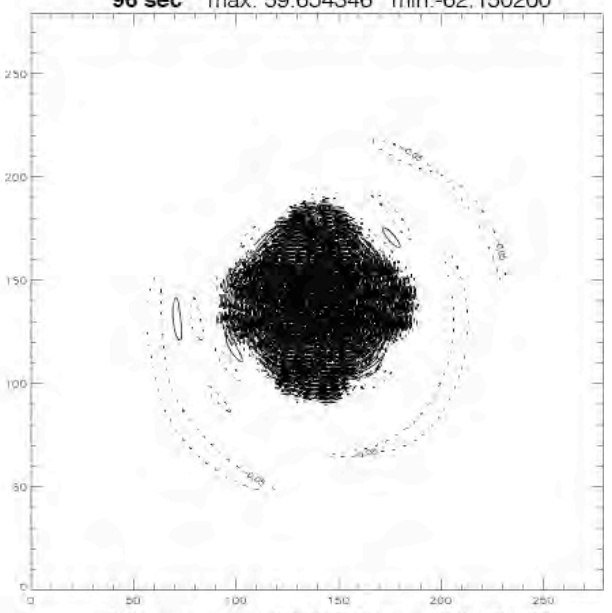

$168 \mathrm{sec} \max : 86.212486 \min :-85.507706$

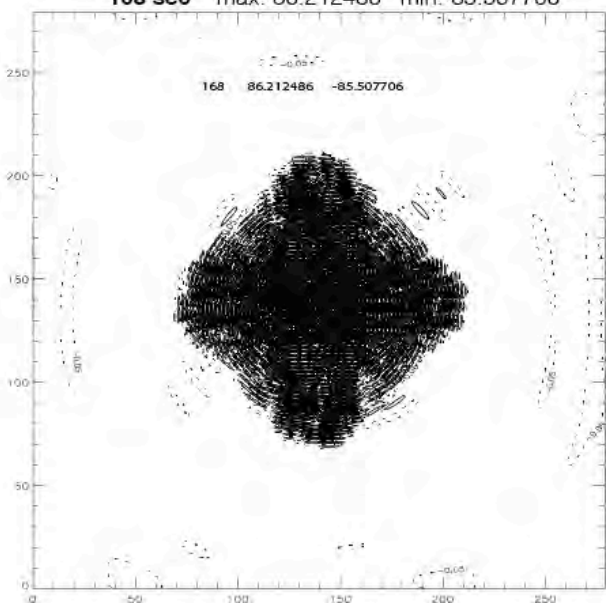

Fig. RF2.

Numerical simulations with noisy forcing, page 5 
Buoyancy generated by a grid-scale random forcing pattern

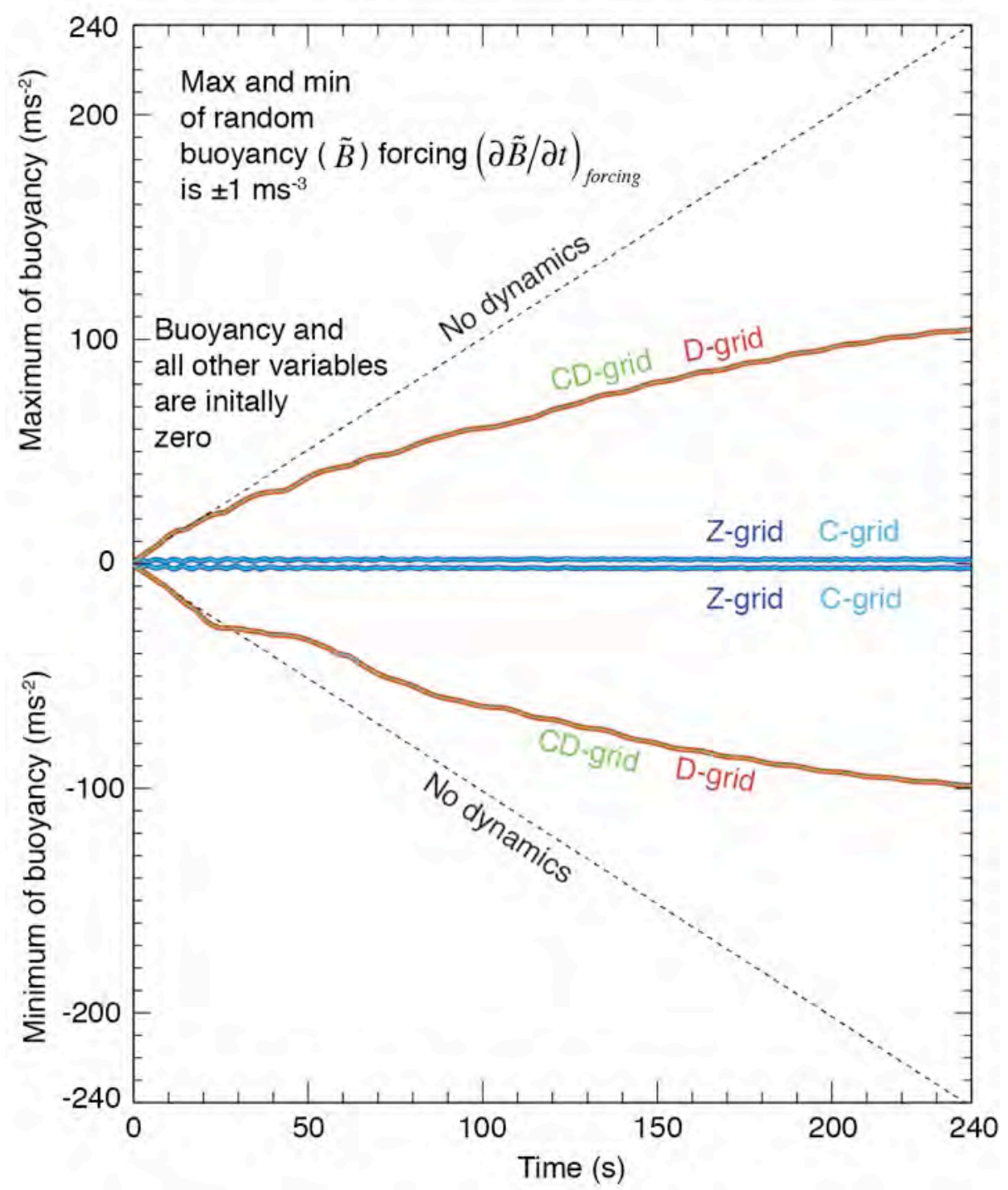

Fig. RF3. 


\section{Horizontal momentum modes with the $A, E$ and $B$ grids}

\section{Continuous solution:}

In this section, we derive the continuous and discrete dispersion equations using the momentum equations instead of the vorticity and divergence equations. So far we examined the modes that are governed by the vorticity and divergence dynamics. Now we examine the modes that are governed by the momentum dynamics. Our main purpose is to answer the question that whether of not the prediction of horizontal momentum allow additional dynamically inert modes that are on top of those obtained using the vorticity and divergence dynamics.

From (1.18)-(1.22) we write

$$
\begin{gathered}
\frac{\partial u}{\partial t}=-\frac{\partial P}{\partial x}+f v \\
\frac{\partial v}{\partial t}=-\frac{\partial P}{\partial x}-f u \\
{\left[\frac{\partial^{2}}{\partial z^{2}}-\left(\frac{1}{2 \rho_{0}} \frac{\partial \rho_{0}}{\partial z}\right)^{2}\right] \frac{\partial P}{\partial t}=\left(\frac{\partial^{2}}{\partial t^{2}}+N^{2}\right)\left(\frac{\partial u}{\partial x}+\frac{\partial v}{\partial y}\right) .}
\end{gathered}
$$

By replacing $P$ by $g h$, and $-\left(\frac{\partial^{2}}{\partial t^{2}}+N^{2}\right) /\left[\frac{\partial^{2}}{\partial z^{2}}-\left(\frac{1}{2 \rho_{0}} \frac{\partial \rho_{0}}{\partial z}\right)^{2}\right]$ by $g H$, we obtain a system of equations that resembles to the shallow-water system. Then we can write

$$
\begin{gathered}
\frac{\partial u}{\partial t}=-g \frac{\partial h}{\partial x}+f v \\
\frac{\partial v}{\partial t}=-g \frac{\partial h}{\partial x}-f u \\
\frac{\partial h}{\partial t}=-H\left(\frac{\partial u}{\partial x}+\frac{\partial v}{\partial y}\right) .
\end{gathered}
$$

From these equations we obtain 


$$
\left(\frac{\partial^{2}}{\partial t^{2}}+f^{2}\right) \frac{\partial u}{\partial t}=g H\left(\frac{\partial^{2}}{\partial x^{2}}+\frac{\partial^{2}}{\partial y^{2}}\right) \frac{\partial u}{\partial t} \quad\left[\text { and }\left(\frac{\partial^{2}}{\partial t^{2}}+f^{2}\right) \frac{\partial v}{\partial t}=g H\left(\frac{\partial^{2}}{\partial x^{2}}+\frac{\partial^{2}}{\partial y^{2}}\right) \frac{\partial v}{\partial t}\right]
$$

Eq. (Mom.7) produces

$$
v\left[v^{2}-f^{2}-g H\left(k^{2}+\ell^{2}\right)\right]=0 .
$$

Eq. (Mom.8) yields $v=0$ solution because the amplitude of either $u$ or $v$ can be zero while an inertia-gravity wave propagate. The divergence produces $v^{2}-f^{2}-g H\left(k^{2}+\ell^{2}\right)=0$.

\section{A-grid:}

The discretization of (Mom.4)-(Mom.6) on the A-grid yields

$$
\begin{gathered}
\frac{\partial u_{i, j}}{\partial t}=-g \frac{h_{i+1, j}-h_{i-1, j}}{2 d}+f v_{i, j}, \\
\frac{\partial v_{i, j}}{\partial t}=-g \frac{h_{i, j+1}-h_{i, j-1}}{2 d}-f u_{i, j}, \\
\frac{\partial h_{i, j}}{\partial t}=-H \frac{u_{i+1, j}-u_{i-1, j}}{2 d}-H \frac{v_{i, j+1}-v_{i, j-1}}{2 d} .
\end{gathered}
$$

Then using

$$
\begin{gathered}
\frac{\partial^{2} u_{i, j}}{\partial t^{2}}=-g \frac{\partial}{\partial t} \frac{h_{i+1, j}-h_{i-1, j}}{2 d}+f \frac{\partial v_{i, j}}{\partial t} \\
\frac{\partial}{\partial t}\left(\frac{h_{i+1, j}-h_{i-1, j}}{2 d}\right)=-H \frac{u_{i+2, j}-2 u_{i, j}+u_{i-2, j}}{4 d^{2}}-H \frac{\left(v_{i+1, j+1}-v_{i-1, j+1}\right)-\left(v_{i+1, j-1}-v_{i-1, j-1}\right)}{4 d^{2}}
\end{gathered}
$$




$$
\begin{aligned}
\frac{\partial}{\partial t} \frac{\left(v_{i+1, j+1}-v_{i-1, j+1}\right)}{4 d^{2}}-\left(v_{i+1, j-1}-v_{i-1, j-1}\right) & \\
-g\left(\frac{h_{i+1, j+2}-h_{i+1, j}-h_{i-1, j+2}+h_{i-1, j}}{8 d^{3}}-\right. & \left.\frac{h_{i+1, j}-h_{i+1, j-2}-h_{i-1, j}+h_{i-1, j-2}}{8 d^{3}}\right) \\
& -f \frac{\left(u_{i+1, j+1}-u_{i-1, j+1}\right)-\left(u_{i+1, j-1}-u_{i-1, j-1}\right)}{4 d^{2}}
\end{aligned}
$$

and (Mom.A.2), we obtain

$$
\left(\frac{\partial^{2}}{\partial t^{2}}+f^{2}\right) \frac{\partial u_{i, j}}{\partial t}=g H \frac{\partial}{\partial t}\left(\frac{u_{i+2, j}+u_{i-2, j}+u_{i, j+2}+u_{i, j-2}-4 u_{i, j}}{4 d^{2}}\right)
$$

Similarly

$$
\left(\frac{\partial^{2}}{\partial t^{2}}+f^{2}\right) \frac{\partial v_{i, j}}{\partial t}=g H \frac{\partial}{\partial t}\left(\frac{v_{i+2, j}+v_{i-2, j}+v_{i, j+2}+v_{i, j-2}-4 v_{i, j}}{4 d^{2}}\right) .
$$

From (Mom.A.7) and (Mom.A.8), the dispersion equation for $u$ and $v$ is

$$
v\left[v^{2}-f^{2}-g H\left(\tilde{\xi}^{2} k^{2}+\tilde{\eta}^{2} \ell^{2}\right)\right]=0
$$

The definitions of $\tilde{\xi}$ and $\tilde{\eta}$ are given by (14b) in A-grid section. For $d \rightarrow 0$, Eq. (Mom.A.9) is identical to the continuous dispersion equation given by (Mom.8). From ( $7^{\prime}$ ) in A-grid section, we can write

$$
\left(\frac{\partial^{2}}{\partial t^{2}}+f^{2}\right) D_{i, j}=g H\left(\frac{D_{i+2, j}+D_{i-2, j}+D_{i, j+2}+D_{i, j-2}-4 D_{i, j}}{4 d^{2}}\right)
$$

for the divergence. The momentum and divergence have the same dispersion equations except the the momentum has an unmoving mode $(v=0)$. The prediction of momentum on the A-grid does not allow any dynamically inert modes on top of the divergence modes.

\section{E-grid:}

The discretization of (Mom.4)-(Mom.6) on the E-grid yields 
For integer mass points:

$$
\begin{gathered}
\frac{\partial u_{i+1 / 2, j}}{\partial t}=-g \frac{h_{i+1, j}-h_{i, j}}{d}+f v_{i+1 / 2, j} \\
\frac{\partial v_{i+1 / 2, j}}{\partial t}=-g \frac{h_{i+1 / 2, j+1 / 2}-h_{i+1 / 2, j-1 / 2}}{d}-f u_{i+1 / 2, j} \\
\frac{\partial h_{i, j}}{\partial t}=-H \frac{u_{i+1 / 2, j}-u_{i-1 / 2, j}}{d}-H \frac{v_{i, j+1 / 2}-v_{i, j-1 / 2}}{d}
\end{gathered}
$$

For half-integer mass points:

$$
\begin{gathered}
\frac{\partial u_{i, j+1 / 2}}{\partial t}=-g \frac{h_{i+1 / 2, j+1 / 2}-h_{i-1 / 2, j+1 / 2}}{d}+f v_{i, j+1 / 2} \\
\frac{\partial v_{i, j+1 / 2}}{\partial t}=-g \frac{h_{i, j+1}-h_{i, j}}{d}-f u_{i, j+1 / 2} \\
\frac{\partial h_{i+1 / 2, j+1 / 2}}{\partial t}=-H \frac{u_{i+1, j+1 / 2}-u_{i, j+1 / 2}}{d}-H \frac{v_{i+1 / 2, j+1}-v_{i+1 / 2, j}}{d}
\end{gathered}
$$

Using (Mom.A.1) and (Mom.A.3), we derive

$$
\frac{\partial^{2} u_{i+1 / 2, j}}{\partial t^{2}}=g H \frac{u_{i+3 / 2, j}-2 u_{i+1 / 2, j}-u_{i-1 / 2, j}}{d^{2}}+g H \frac{\left(v_{i+1, j+1 / 2}-v_{i, j+1 / 2}\right)-\left(v_{i+1, j-1 / 2}-v_{i, j-1 / 2}\right)}{d^{2}}+f \frac{\partial v_{i+1 / 2, j}}{\partial t}
$$

(Mom.E.7)

From (Mom.A.5), we write

$$
\begin{aligned}
& \frac{\partial}{\partial t} \frac{\left(v_{i+1, j+1 / 2}-v_{i, j+1 / 2}\right)-\left(v_{i+1, j-1 / 2}-v_{i, j-1 / 2}\right)}{d^{2}}= \\
& -g \frac{\left(h_{i+1, j+1}-2 h_{i+1, j}+h_{i+1, j-1}\right)-\left(h_{i, j+1}-2 h_{i, j}+h_{i, j-1}\right)}{d^{3}}-f \frac{\left(u_{i+1, j+1 / 2}-u_{i, j+1 / 2}\right)-\left(u_{i+1, j-1 / 2}-u_{i, j-1 / 2}\right)}{d^{2}}
\end{aligned}
$$

From (Mom.A.5), we write 


$$
\begin{aligned}
& \frac{\partial}{\partial t}\left(\frac{h_{i+1 / 2, j+1 / 2}-h_{i+1 / 2, j-1 / 2}}{d}\right)= \\
& \quad-H \frac{u_{i+1, j+1 / 2}-u_{i, j+1 / 2}}{d^{2}}+H \frac{u_{i+1, j-1 / 2}-u_{i, j-1 / 2}}{d^{2}}-H \frac{v_{i+1 / 2, j+1}-v_{i+1 / 2, j}}{d^{2}}+H \frac{v_{i+1 / 2, j}-v_{i+1 / 2, j-1}}{d^{2}}
\end{aligned}
$$

Using (Mom.A.2), (Mom.A.7), (Mom.A.8), and (Mom.A.9), we derive the wave equation

$$
\left(\frac{\partial^{2}}{\partial t^{2}}+f^{2}\right) \frac{\partial}{\partial t} u_{i+1 / 2, j}=g H \frac{\partial}{\partial t}\left(\frac{u_{i+3 / 2, j}+u_{i+1 / 2, j+1}+u_{i+1 / 2, j-1}+u_{i-1 / 2, j}-4 u_{i+1 / 2, j}}{d^{2}}\right) .
$$

Similarly

$$
\begin{gathered}
\left(\frac{\partial^{2}}{\partial t^{2}}+f^{2}\right) \frac{\partial}{\partial t} u_{i, j+1 / 2}=g H \frac{\partial}{\partial t}\left(\frac{u_{i+1, j+1 / 2}+u_{i-1, j+1 / 2}+u_{i, j+3 / 2}+u_{i, j-1 / 2}-4 u_{i, j+1 / 2}}{d^{2}}\right) \\
\left(\frac{\partial^{2}}{\partial t^{2}}+f^{2}\right) \frac{\partial v_{i+1 / 2, j}}{\partial t}=g H \frac{\partial}{\partial t}\left(\frac{v_{i+1 / 2, j+1}+v_{i+1 / 2, j-1}+v_{i+3 / 2, j}+v_{i-1 / 2, j}-4 v_{i+1 / 2, j}}{d^{2}}\right) \\
\left(\frac{\partial^{2}}{\partial t^{2}}+f^{2}\right) \frac{\partial v_{i, j+1 / 2}}{\partial t}=g H \frac{\partial}{\partial t}\left(\frac{v_{i+1, j+1 / 2}+v_{i-1, j+1 / 2}+v_{i, j+3 / 2}+v_{i-1 / 2, j-1 / 2}-4 v_{i, j+1 / 2}}{d^{2}}\right) .
\end{gathered}
$$

These equations yield the following dispersion equation

$$
v\left[v^{2}-f^{2}-g H\left(\xi^{2} k^{2}+\eta^{2} \ell^{2}\right)\right]=0 \quad k_{\max }=\ell_{\max }=2 \pi / d
$$

For $d \rightarrow 0$, Eq. (Mom.A.14) is identical to the continuous dispersion equation given by (Mom. 8). The momentum and divergence have the same dispersion equations except the momentum has an unmoving mode $(v=0)$. The prediction of momentum on the E-grid does not allow any additional dynamically inert modes on top of the divergence modes.

\section{B-grid:}

The discretization of (Mom.4)-(Mom.6) on the B-grid yields

$$
\frac{\partial u_{i, j}}{\partial t}=-g \frac{h_{i+1 / 2, j+1 / 2}+h_{i+1 / 2, j-1 / 2}-h_{i-1 / 2, j+1 / 2}-h_{i-1 / 2, j-1 / 2}}{2 d}+f v_{i, j}
$$




$$
\begin{gathered}
\frac{\partial v_{i, j}}{\partial t}=-g \frac{h_{i+1 / 2, j+1 / 2}+h_{i-1 / 2, j+1 / 2}-h_{i+1 / 2, j-1 / 2}-h_{i-1 / 2, j-1 / 2}}{2 d}-f u_{i, j} \\
\frac{\partial h_{i+1 / 2, j+1 / 2}}{\partial t}=-H \frac{u_{i+1, j+1}+u_{i+1, j}-u_{i, j+1}-u_{i, j}}{2 d}-H \frac{v_{i+1, j+1}+v_{i, j+1}-v_{i+1, j}-v_{i, j}}{2 d}
\end{gathered}
$$

From these equations, we derive

$$
\begin{array}{r}
\frac{\partial^{2} u_{i, j}}{\partial t^{2}}=g H \frac{\left(u_{i+1, j+1}+2 u_{i+1, j}+u_{i+1, j-1}\right)-2\left(u_{i, j+1}+2 u_{i, j}+u_{i, j-1}\right)+\left(u_{i-1, j+1}+2 u_{i-1, j}+u_{i-1, j-1}\right)}{4 d^{2}} \\
+g H \frac{\left(v_{i+1, j+1}-v_{i+1, j-1}\right)-\left(v_{i-1, j+1}-v_{i-1, j-1}\right)}{4 d^{2}}+f \frac{\partial v_{i, j}}{\partial t}
\end{array}
$$

and

$$
\begin{aligned}
& \frac{\partial}{\partial t} \frac{\left(v_{i+1, j+1}-v_{i+1, j-1}\right)-\left(v_{i-1, j+1}-v_{i-1, j-1}\right)}{4 d^{2}}=-g \frac{h_{i+3 / 2, j+3 / 2}+h_{i+1 / 2, j+3 / 2}-h_{i+3 / 2, j+1 / 2}-h_{i+1 / 2, j+1 / 2}}{8 d^{3}} \\
& +g \frac{h_{i+3 / 2, j-1 / 2}+h_{i+1 / 2, j-1 / 2}-h_{i+3 / 2, j-3 / 2}-h_{i+1 / 2, j-3 / 2}}{8 d^{3}}+g \frac{h_{i-1 / 2, j+3 / 2}+h_{i-3 / 2, j+3 / 2}-h_{i-1 / 2, j+1 / 2}-h_{i-3 / 2, j+1 / 2}}{8 d^{3}} . \\
& -g \frac{h_{i-1 / 2, j-1 / 2}+h_{i-3 / 2, j-1 / 2}-h_{i-1 / 2, j-3 / 2}-h_{i-3 / 2, j-3 / 2}}{8 d^{3}}-f\left(\frac{u_{i+1, j+1}-u_{i+1, j-1}-u_{i-1, j+1}+u_{i-1, j-1}}{4 d^{2}}\right)
\end{aligned}
$$

Using (Mom.B.2) and (Mom.B.5) in (Mom.B.4) and after lengthly derivations, we obtain

$$
\left(\frac{\partial^{2}}{\partial t^{2}}+f^{2}\right) \frac{\partial u_{i, j}}{\partial t}=g H \frac{\partial}{\partial t}\left(\frac{u_{i+1, j+1}+u_{i+1, j-1}+u_{i-1, j+1}+u_{i-1, j-1}-4 u_{i, j}}{2 d^{2}}\right) .
$$

Similarly,

$$
\left(\frac{\partial^{2}}{\partial t^{2}}+f^{2}\right) \frac{\partial v_{i, j}}{\partial t}=g H \frac{\partial}{\partial t}\left(\frac{v_{i+1, j+1}+v_{i+1, j-1}+v_{i-1, j+1}+v_{i-1, j-1}-4 v_{i, j}}{2 d^{2}}\right)
$$

Then the dispersion equation is

$$
v\left[v^{2}-f^{2}-g H\left(\xi^{2} k^{2}+\eta^{2} \ell^{2}-\frac{1}{2} d^{2} \xi^{2} k^{2} \eta^{2} \ell^{2}\right)\right]=0 .
$$


For $d \rightarrow 0$, Eq. (Mom.B.8) is identical to the continuous dispersion equation given by (Mom.8). The momentum and divergence have the same dispersion equations except the momentum has an unmoving mode $(v=0)$. The prediction of momentum on the B-grid does not allow any additional dynamically inert modes on top of the divergence modes. 